\title{
U.S.DEPARTMENT OF THE INTERIOR
}

U.S. GEOLOGICAL SURVEY

\section{QUANTITATIVE GEOCHEMISTRY OF ROCKS FROM THE ADELAIDE MINING DISTRICT, HUMBOLDT COUNTY, NEVADA}

By

THERESA M. COOKRO ${ }^{1}$

OPEN FILE REPORT 93-249

This report is preliminary and has not been reviewed for conformity with U.S. Geological Survey editorial standards (or with the North American Stratigraphic Code). Any use of trade, product, or firm names is for descriptive purposes only and does not imply endorsement by the U.S. Government.

${ }^{1}$ U.S. GEOLOGICAL SURVEY

BOX 250546 MS 937

DENVER FEDERAL CENTER

LAKEWOOD, COLORADO 80225 
INTRODUCTION.

ADELAIDE MINING

DISTRICT

ACKNOWLEDGEMENTS......................................................

REFERENCES.

Sample number and locations in numeric order and $\mathrm{Hg}, \mathrm{Ag}, \mathrm{Au}\left({ }^{*}\right.$ Atomic absorption spectrometry: solvent extraction and hydride generation)

Sample number and locations sorted by sample type and $\mathrm{Hg}, \mathrm{Ag}, \mathrm{Au}$ (*Atomic absorption spectrometry: solvent extraction and hydride generation)

$\mathrm{Al}, \mathrm{Ca}, \mathrm{Fe}, \mathrm{K}, \mathrm{Mg}, \mathrm{Na}, \mathrm{P}, \mathrm{Ti}, \mathrm{Mn}, \mathrm{Ag}, \mathrm{As}, \mathrm{Au}, \mathrm{B}, \mathrm{Ba}, \mathrm{Be}, \mathrm{Bi}, \mathrm{Cd}, \mathrm{Ce}, \mathrm{Co}$, $\mathrm{Cr}, \mathrm{Cu}, \mathrm{Eu}, \mathrm{Ga}, \mathrm{Ge}, \mathrm{Ho}, \mathrm{La}, \mathrm{Li}, \mathrm{Mo}, \mathrm{Nb}, \mathrm{Nd}, \mathrm{Ni}, \mathrm{Pb}, \mathrm{Sc}, \mathrm{Sn}, \mathrm{Sr}, \mathrm{Ta}, \mathrm{Th}$, $\mathrm{U}, \mathrm{V}, \mathrm{W}, \mathrm{Y}, \mathrm{Yb}, \mathrm{Zn}, \mathrm{Zr}$ (*Inductively coupled plasma-atomic emission spectrometry )

$\mathrm{Fe}, \mathrm{Na}, \mathrm{Ba}, \mathrm{Co}, \mathrm{Cr}, \mathrm{Cs}, \mathrm{Hf}, \mathrm{Rb}, \mathrm{Sb}, \mathrm{Ta}, \mathrm{Th}, \mathrm{U}, \mathrm{Zn}, \mathrm{Zr}, \mathrm{Sc}, \mathrm{La}, \mathrm{Ce}, \mathrm{Nd}, \mathrm{Sm}$, $\mathrm{Eu}, \mathrm{Tb}, \mathrm{Yb}, \mathrm{Lu}$ (*Radiochemical neutron activation analysis).

$\mathrm{Pt}, \mathrm{Pd}, \mathrm{Rh}, \mathrm{Ru}, \mathrm{Ir}$ (*Instrumental neutron activation analysis).

As, Hg, Se, Te, Tl, W, Au (*Inductively coupled plasma-atomic emission spectrometry (partial).

Sn, Te, Tl (*Atomic absorption spectrometry: solvent extraction).........154

Cl, F (*Atomic absorption spectrometry: ion exchange chromatography).

*See "Baedecker, P.A., 1987, Methods for Geochemical Analysis, U.S. Geological Survey Bulletin 1770" for more information on the geochemical methods of analysis.. 


\section{INTRODUCTION}

Geochemical analysis of rocks sampled from the Adelaide mining district in 1985-86 was completed in 1990; the length of time for completion was partly due to using quantitative rather than quicker semiquantitative analytical methods, a move in laboratory facilities at the USGS, and budget limitations. Due to other commitments the author has not been able to work on interpretation of the data.

\section{ADELAIDE MINING DISTRICT}

The Adelaide Mining District, also called Gold Run, is located $16 \mathrm{~km}$ south of the town of Golconda, Nevada in the eastern foothills of the Sonoma Range, Humboldt County Nevada (fig. 1). The District produced at the turn of the century, 1920's and 1950's by underground and open pit methods (Wilden, 1964; Trengove; 1959; Vanderberg, 1938; Stager and Tingley, 1988). In the late 1980's the area was open pit mined for bulk minable gold. Generally the district produced gold, silver, copper, lead and zinc. There is a placer gold operation on Gold Run Creek, one of several generally intermittent stream valleys that drain the area. Also included in the district are: the Adelaide Crown Mines, mostly epithermal precious metal areas; the Adelaide Mine with skarn-related metals ( $\mathrm{Cu}, \mathrm{Zn}, \mathrm{W}, \mathrm{Ag}$ ); small manganese mines (Penrose, 1983); old gold placers (along Cumberland Creek and in Bill Major's Canyon) and the Gold Run Placer.

Lower Cambrian to Lower Ordovician Preble Formation (Rees and Rowell, 1980; Rowell and others, 1979; Hotz and Willden, 1964 and Gilluly, 1967) host the bulk of the mineralization. The rock is strongly faulted and silicified which makes it difficult to determine pre-alteration lithologies. Skarn is present in the Preble Formation at both the Adelaide Crown Mines and the Adelaide Mine, but the greater part of the skarn in the district is in the vicinity of the Adelaide mine. 
The purpose of the study is to ascertain the distribution of major and trace elements in the district, and with supplemental petrographic data, identify elemental patterns of various additions to and subtractions from the metallogenic environments: epithermal, skarn, and the probable hot spring environment that produced the manganese.

See Cookro and Theodore (1989) for further information on the Adelaide Mining district.

\section{ACKNOWLEDGEMENTS}

My thanks to the following USGS project leaders and analysts:

Solvent Extraction and Bydride Generation (Hg, Ag, Au) : S.A. Wilson, C. Gent, T. McCollom, K. Kennedy, J.G. Crock, P.H. Briggs, D.E. Detra, and M. Malcolm.

Optical Spectroscopy: P. H. Briggs, M. Malcolm, and D.E. Detra,

Radiochemistry: D.M., McKown, J. Budahn, R. Knight, D. Mckown, C. Palmer, J. Grossman, and R.B. Vaughn,

Ion Chromatography $(\mathrm{Cl}, \mathrm{Fl})$ : W.H. Ficklin, J. Sharkey, C. Papp, L.L. Jackson, S. Roof, and E.L. Brandt,

Solvent extraction ( $\mathrm{Sn}, \mathrm{Te}, \mathrm{Th}): \mathrm{P}$. Aruscavage, M. Doughten, R. Moore, B. Libby, and J. Evans,

ICP Mass Spectrometry: (Pt, $P d, R h, R u, I r)$ : A.L. Meier and R. Carlson

Instrumental Neutron Activation Analysis: (As, Hg, Se, Te, Tl, W, Au) R.M. O'Leary, K.Kennedy, P. Hageman, E. Welsch, F. Tippit, and R. Roemer 


\section{REFERENCES}

Baedecker P.A., ed., 1987, Methods for geochemical analysis: U.S. Geological Survey Bulletin 1770, p. A1-K5.

Cookro, T.M., and Theodore, T.G., 1989, Gold, silver, and mercury rock chemistry for the Adelaide Mining district, Sonoma Range, Humboldt county, Nevada, $47 \mathrm{p}$.

Gilluly, James, and Gates, Olcott, Tectonic and igneous geology of the northern Shoshone Range, Nevada: U.S. Geological Survey Professional Paper 465, 153 p.

Hotz, P.E., and Willden, R., 1964, Geology and mineral deposits of the Osgood Mountains quadrangle, Humboldt County, Nevada: U.s. Geological Survey Professional Paper 431, 128 p.

Penrose, R.A.F., 1893, A Pleistocene manganese deposit near Golconda, Nevada: Journal of Geology, v.1, p. 275-282.

Rees, M.N., and Rowell, A.J., 1980, Preble Formation, a Cambrian outer continental shelf deposit in Nevada: Brigham Young University Geology studies, v. 27, pt.1, p. 1-8.

Rowell, A.J., Rees, M.N., and Suczek, C.A., 1979, Margin of the North American Continent in Nevada during Late Cambrian time: American Journal of Science, v. 279, p. 1-17.

Stager, H.K., and Tingley, J.V., 1988, Tungsten deposits in Nevada: Nevada Bureau of Mines and Geology Bulletin 105, 256 p.

Trengove, R.R., 1959, Reconnaissance of Nevada manganese deposits: U.S. Bureau of Mines Report of Investigations 5446.

Vanderburg, W.O., 1938, Reconnaissance of mining districts in Humboldt county, Nevada U.s. Bureau of Mines, Information circular, 6995, $54 \mathrm{p}$.

Willden, C.R., 1965, Geology and mineral deposits of Humboldt County, Nevada: Nevada Bureau of Mines Bulletin, v. 59, 154 p. 
Table 1: Rock Geochemistry of the Adelaide Mining District, Humboldt County Nevada. Cookro and Theodore, 1989

\begin{tabular}{llllrc} 
Sample & \multicolumn{2}{c}{ Location in UTMS } & Hg & Ag & $\mathrm{Au}$ \\
85KG001 & 57.57119 & 17.61918 & 0.06 & 3 & 0.1 \\
85KG002 & 57.57119 & 17.61918 & 0.06 & 3 & 0.1 \\
85KG003 & 57.17606 & 17.95710 & 0.24 & 3 & 0.1 \\
85KG004 & 57.17606 & 17.95710 & 0.17 & 3 & 0.1 \\
85KG005 & 57.16234 & 17.93342 & 0.21 & 3 & 0.1 \\
85KG006 & 57.16234 & 17.93342 & 0.21 & 3 & 0.1 \\
85KG007 & 56.98806 & 17.88432 & 0.07 & 3 & 0.1 \\
85KG008 & 56.95602 & 18.04011 & 0.07 & 3 & 0.1 \\
85KG009 & 56.95602 & 18.04011 & 0.02 & 3 & 0.1 \\
85KG010 & 56.95602 & 18.04011 & 0.52 & 3 & 0.1 \\
85KG011 & 56.91622 & 18.11586 & 0.07 & 3 & 0.1 \\
85KG012 & 56.91622 & 18.11586 & 0.15 & 4 & 0.1 \\
85KG013 & 56.92472 & 18.43320 & 0.04 & 3 & 0.1 \\
85KG014 & 56.92472 & 18.43320 & 0.03 & 3 & 0.1 \\
85KG015 & 57.05797 & 18.46572 & 0.00 & 13 & 70.0 \\
85KG016 & 57.05797 & 18.46572 & 0.02 & 3 & 0.3 \\
85KG017 & 56.90713 & 18.84876 & 0.00 & 12 & 0.1 \\
85KG018 & 56.90713 & 18.84876 & 0.00 & 24 & 0.1 \\
85KG019 & 57.06220 & 18.68389 & 0.28 & 3 & 0.1 \\
85KG020 & 56.38609 & 18.06224 & 0.40 & 3 & 0.1 \\
85KG021 & 56.38609 & 18.06224 & 0.74 & 3 & 0.1 \\
85KG022 & 56.02438 & 18.16545 & 0.89 & 3 & 0.1 \\
85KG023 & 56.02438 & 18.16545 & 0.00 & 8 & 0.3 \\
85KG024 & 55.93018 & 18.18320 & 0.70 & 3 & 0.1 \\
85KG025 & 55.93018 & 18.18320 & 0.00 & 7 & 0.3 \\
85KG026 & 55.91488 & 18.22316 & 0.00 & 7 & 0.2 \\
85KG027 & 55.91488 & 18.22316 & 0.29 & 3 & 0.1 \\
85KG028 & 55.44527 & 18.36754 & 0.13 & 3 & 0.1 \\
85KG029 & 55.38828 & 18.93469 & 1.20 & 3 & 0.1 \\
85KG030 & 55.39885 & 19.13334 & 2.00 & 4 & 0.1 \\
85KG031 & 55.39885 & 19.13334 & 0.00 & 19 & 0.1 \\
85KG032 & 55.39885 & 19.13334 & 0.53 & 7 & 0.1 \\
85KG033 & 55.40350 & 19.07089 & 0.27 & 4 & 0.1 \\
85KG034 & 55.40350 & 19.07089 & 0.00 & 15 & 0.1 \\
85KG035 & 55.37235 & 18.88290 & 1.20 & 3 & 0.1 \\
85KG036 & 55.40460 & 18.88004 & 0.51 & 3 & 0.1
\end{tabular}




\begin{tabular}{llllrl} 
Sample & \multicolumn{2}{l}{ Location in UTMS } & $\mathrm{Hg}$ & $\mathrm{Ag}$ & $\mathrm{Au}$ \\
& & & & & \\
85KG037 & 55.40460 & 18.88004 & 0.03 & 3 & 0.1 \\
85KG038 & 55.52324 & 18.77695 & 0.04 & 3 & 0.1 \\
85KG039 & 55.52324 & 18.77695 & 0.07 & 3 & 0.1 \\
85KG041 & 54.80167 & 18.43835 & 0.10 & 3 & 0.1 \\
85KG042 & 54.90145 & 18.47435 & 0.76 & 3 & 0.1 \\
85KG044 & 55.42118 & 18.68918 & 3.30 & 3 & 0.3 \\
85KG045 & 54.78681 & 17.05312 & 0.17 & 3 & 0.1 \\
85KG046 & 54.78681 & 17.05312 & 0.03 & 3 & 0.1 \\
85KG048 & 55.04788 & 17.53353 & 0.29 & 3 & 0.1 \\
85KG049 & 55.04788 & 17.53353 & 0.34 & 3 & 0.1 \\
85KG050 & 54.87268 & 17.23770 & 0.23 & 3 & 0.1 \\
85KG051 & 54.87268 & 17.23770 & 0.08 & 3 & 0.1 \\
85KG052 & 55.59356 & 17.52027 & 0.00 & 97 & 3.5 \\
85KG053 & 55.59356 & 17.52027 & 0.00 & 20 & 0.4 \\
85KG054 & 55.59356 & 17.52027 & 0.00 & 15 & 0.4 \\
85KG055 & 55.52160 & 17.42335 & 0.00 & 15 & 0.9 \\
85KG057 & 55.83339 & 17.64192 & 0.29 & 3 & 0.1 \\
85KG058 & 55.81063 & 17.67096 & 0.00 & 57 & 0.7 \\
85KG059 & 55.81063 & 17.67096 & 0.00 & 33 & 0.1 \\
85KG060 & 55.81063 & 17.67096 & 0.00 & 38 & 0.5 \\
85KG061 & 55.59619 & 17.80772 & 0.11 & 5 & 0.1 \\
85TC002 & 58.34813 & 17.04885 & 0.06 & 3 & 0.1 \\
85TC005 & 58.43006 & 16.98712 & 0.00 & 40 & 0.1 \\
85TC048 & 55.04788 & 17.53353 & 0.02 & 4 & 0.1 \\
85TC049 & 55.04788 & 17.53353 & 0.07 & 3 & 0.1 \\
85TC051 & 57.11113 & 16.01912 & 0.00 & 3 & 0.1 \\
85TC054 & 56.81601 & 16.13354 & 0.00 & 28 & 0.1 \\
85TC055 & 58.27589 & 16.94626 & 0.02 & 3 & 0.1 \\
85TC056 & 58.27589 & 16.94626 & 0.02 & 3 & 0.1 \\
85TC060 & 58.27589 & 16.94626 & 0.00 & 110 & 0.1 \\
85TC061 & 58.27589 & 16.94626 & 0.06 & 80 & 0.2 \\
85TC064 & 58.27589 & 16.94626 & 0.07 & 46 & 0.1 \\
85TC065 & 57.06367 & 18.71031 & 0.34 & 3 & 0.1 \\
85TC067 & 57.06367 & 18.71031 & 0.04 & 3 & 0.1 \\
85TC068 & 57.06367 & 18.71031 & 0.03 & 3 & 0.1 \\
85TC069 & 57.08014 & 18.75719 & 0.09 & 3 & 0.1 \\
85TC070 & 57.08014 & 18.75719 & 0.06 & 3 & 0.1 \\
85TC071 & 57.06349 & 18.83326 & 0.03 & 3 & 0.1
\end{tabular}




\begin{tabular}{llllrr} 
Sample & \multicolumn{2}{c}{ Location in UTMS } & Hg & Ag & Au \\
& & & & & \\
85TC072 & 57.06349 & 18.83326 & 0.07 & 3 & 0.1 \\
85TC073 & 57.06349 & 18.83326 & 0.02 & 3 & 0.1 \\
85TC074 & 57.06349 & 18.83326 & 0.05 & 3 & 0.1 \\
85TC075 & 57.06349 & 18.83326 & 0.72 & 5 & 0.1 \\
85TC076 & 57.12843 & 18.90581 & 0.03 & 3 & 0.1 \\
85TC077 & 57.08815 & 18.62680 & 0.25 & 3 & 0.1 \\
85TC078 & 57.30080 & 18.67540 & 0.03 & 3 & 0.1 \\
85TC079 & 57.39147 & 18.66748 & 0.02 & 3 & 0.1 \\
85TC080 & 57.39147 & 18.66748 & 0.02 & 3 & 0.1 \\
85TC081 & 57.90168 & 18.88911 & 0.05 & 3 & 10.0 \\
85TC082 & 57.90168 & 18.88911 & 0.02 & 3 & 0.1 \\
85TC083 & 57.93262 & 18.80987 & 0.02 & 3 & 0.1 \\
85TC084 & 56.84576 & 18.95232 & 0.18 & 3 & 0.2 \\
85TC085 & 57.06134 & 19.12811 & 0.08 & 3 & 0.1 \\
85TC086 & 57.06134 & 19.12811 & 0.03 & 3 & 0.1 \\
85TC087 & 57.06134 & 19.12811 & 0.12 & 3 & 0.1 \\
85TC088 & 57.05112 & 19.09276 & 0.00 & 8 & 0.1 \\
85TC089 & 57.05112 & 19.09276 & 0.00 & 8 & 0.1 \\
85TC090 & 57.05112 & 19.09276 & 0.00 & 27 & 0.1 \\
85TC091 & 57.06183 & 19.07366 & 0.00 & 11 & 0.2 \\
85TC092 & 57.06183 & 19.07366 & 0.05 & 4 & 0.1 \\
85TC093 & 57.09531 & 19.02613 & 0.03 & 3 & 0.1 \\
85TC095 & 57.08729 & 19.01889 & 0.32 & 8 & 0.6 \\
85TC096 & 57.14360 & 19.08360 & 0.02 & 3 & 0.1 \\
85TC097 & 57.14360 & 19.08360 & 0.03 & 3 & 0.1 \\
85TC098 & 57.17725 & 18.95777 & 0.02 & 3 & 0.1 \\
85TC099 & 57.17725 & 18.95777 & 0.02 & 3 & 0.1 \\
85TC100 & 57.17725 & 18.95777 & 0.02 & 3 & 0.1 \\
85TC102 & 55.67828 & 18.34369 & 0.00 & 23 & 0.1 \\
85TC117 & 57.59252 & 17.50710 & 0.02 & 3 & 0.1 \\
85TC118 & 57.59252 & 17.50710 & 0.03 & 3 & 0.1 \\
85TC119 & 57.17606 & 17.95710 & 0.28 & 3 & 0.1 \\
85TC120 & 57.16234 & 17.93342 & 0.07 & 3 & 0.1 \\
85TC121 & 56.97013 & 17.91086 & 0.03 & 3 & 0.1 \\
85TC122 & 56.97281 & 17.97383 & 0.03 & 3 & 0.1 \\
85TC123 & 56.97281 & 17.97383 & 0.03 & 3 & 0.1 \\
85TC124 & 56.97281 & 17.97383 & 0.03 & 3 & 0.1 \\
85TC125 & 56.87669 & 18.16715 & 0.00 & 42 & 0.1
\end{tabular}




\begin{tabular}{llllrl} 
Sample & \multicolumn{2}{l}{ Location in UTMS } & Hg & $\mathrm{Ag}$ & $\mathrm{Au}$ \\
& & & & & \\
85TC126 & 56.87669 & 18.16715 & 0.02 & 3 & 0.1 \\
85TC127 & 56.87669 & 18.16715 & 0.06 & 3 & 0.1 \\
85TC128 & 56.87669 & 18.16715 & 0.12 & 3 & 0.3 \\
85TC129 & 56.90713 & 18.84876 & 0.00 & 110 & 0.3 \\
85TC130 & 56.90713 & 18.84876 & 0.00 & 14 & 0.1 \\
85TC131 & 56.90713 & 18.84876 & 0.00 & 11 & 0.1 \\
85TC132 & 56.38483 & 18.05920 & 0.68 & 3 & 0.1 \\
85TC133 & 56.38483 & 18.05920 & 0.11 & 3 & 0.1 \\
85TC134 & 56.38483 & 18.05920 & 0.31 & 3 & 0.1 \\
85TC134A & 56.38483 & 18.05920 & 0.34 & 7 & 0.1 \\
85TC135 & 56.05713 & 18.23163 & 0.83 & 3 & 0.1 \\
85TC136 & 56.05713 & 18.23163 & 0.18 & 3 & 0.1 \\
85TC137 & 55.91640 & 18.01823 & 0.62 & 3 & 0.1 \\
85TC138 & 55.91640 & 18.01823 & 0.25 & 3 & 0.1 \\
85TC139 & 55.97489 & 17.97281 & 2.60 & 3 & 0.1 \\
85TC141 & 55.48371 & 18.41719 & 0.00 & 9 & 0.3 \\
85TC142 & 55.48371 & 18.41719 & 0.00 & 17 & 0.8 \\
85TC143 & 55.48371 & 18.41719 & 1.00 & 3 & 0.1 \\
85TC147 & 55.34423 & 19.06400 & 0.44 & 3 & 0.1 \\
85TC148 & 55.34423 & 19.06400 & 0.47 & 4 & 0.1 \\
85TC149 & 55.34423 & 19.06400 & 0.00 & 8 & 0.1 \\
85TC150 & 55.34423 & 19.06400 & 3.70 & 5 & 0.1 \\
85TC151 & 55.34423 & 19.06400 & 0.00 & 25 & 0.1 \\
85TC152 & 55.34423 & 19.06400 & 2.30 & 4 & 0.1 \\
85TC153 & 55.34423 & 19.06400 & 0.90 & 3 & 0.1 \\
85TC155 & 55.34423 & 19.06400 & 4.40 & 4 & 0.1 \\
85TC156 & 55.34423 & 19.06400 & 1.20 & 3 & 0.7 \\
85TC157 & 55.42155 & 18.82599 & 0.00 & 31 & 0.2 \\
85TC158 & 55.42155 & 18.82599 & 0.00 & 90 & 0.1 \\
85TC159 & 55.43778 & 18.85513 & 0.00 & 26 & 0.1 \\
85TC160 & 55.43778 & 18.85513 & 0.70 & 3 & 0.1 \\
85TC161 & 55.43778 & 18.85513 & 0.07 & 3 & 0.1 \\
85TC162 & 55.43778 & 18.85513 & 1.60 & 3 & 0.1 \\
85TC163 & 55.43778 & 18.85513 & 8.40 & 3 & 0.4 \\
85TC164 & 55.43778 & 18.85513 & 1.30 & 3 & 0.1 \\
85TC167 & 55.43778 & 18.85513 & 0.19 & 3 & 0.1 \\
85TC168 & 55.43778 & 18.85513 & 0.02 & 3 & 0.1 \\
85TC169 & 55.43778 & 18.85513 & 0.80 & 3 & 0.1
\end{tabular}


Sample Location in UTMS $\mathrm{Hg} \quad \mathrm{Ag} \quad \mathrm{Au}$

$\begin{array}{llllrl}\text { 85TC171 } & 54.78681 & 17.05312 & 0.20 & 3 & 0.1 \\ \text { 85TC172 } & 55.04788 & 17.53353 & 0.46 & 5 & 0.1 \\ \text { 85TC173 } & 55.09430 & 17.22342 & 0.04 & 3 & 0.1 \\ \text { 85TC177 } & 55.49084 & 17.71980 & 0.00 & 10 & 0.1 \\ \text { 85TC178 } & 55.49084 & 17.71980 & 0.00 & 18 & 1.4 \\ \text { 85TC181 } & 55.49084 & 17.71980 & 0.00 & 56 & 8.0 \\ \text { 85TC182 } & 55.49084 & 17.71980 & 0.00 & 14 & 0.1 \\ \text { 85TC184 } & 55.59619 & 17.80772 & 0.00 & 27 & 0.3 \\ \text { 85TC185 } & 55.58628 & 17.65922 & 0.00 & 21 & 0.1 \\ \text { 86TC001 } & 58.08130 & 16.67647 & 0.04 & 6 & 0.1 \\ \text { 86TC002 } & 58.08130 & 16.67647 & 0.00 & 52 & 0.1 \\ \text { 86TC003 } & 58.08130 & 16.67647 & 0.00 & 210 & 0.1 \\ \text { 86TC004 } & 58.08130 & 16.67647 & 0.00 & 18 & 0.1 \\ \text { 86TC005 } & 58.08130 & 16.67647 & 0.00 & 32 & 0.1 \\ \text { 86TC006 } & 58.15807 & 16.88225 & 0.00 & 17 & 0.1 \\ \text { 86TC007 } & 58.16114 & 16.88467 & 0.00 & 33 & 0.1 \\ \text { 86TC008 } & 58.16114 & 16.88467 & 0.04 & 4 & 0.1 \\ \text { 86TC009 } & 57.97242 & 16.81861 & 0.00 & 15 & 0.2 \\ \text { 86TC010 } & 57.97242 & 16.81861 & 0.03 & 4 & 0.1 \\ \text { 86TC011 } & 57.97242 & 16.81861 & 0.02 & 4 & 0.1 \\ \text { 86TC012 } & 58.08008 & 16.67648 & 0.00 & 40 & 0.3 \\ \text { 86TC013 } & 58.08130 & 16.67647 & 0.02 & 4 & 0.1 \\ \text { 86TC014 } & 57.89773 & 16.72325 & 0.02 & 4 & 0.1 \\ \text { 86TC015 } & 58.05893 & 16.62779 & 0.02 & 4 & 0.1 \\ \text { 86TC016 } & 57.98221 & 16.66421 & 0.02 & 4 & 0.1 \\ \text { 86TC017 } & 57.93257 & 16.48878 & 0.02 & 4 & 0.1 \\ \text { 86TC018 } & 57.92890 & 16.48820 & 0.02 & 4 & 0.1 \\ \text { 86TC019 } & 57.92793 & 16.44184 & 0.02 & 4 & 0.1 \\ \text { 86TC020 } & 57.79901 & 16.56841 & 0.80 & 22 & 0.1 \\ \text { 86TC021 } & 57.79901 & 16.56841 & 0.36 & 4 & 0.1 \\ \text { 86TC022 } & 57.73371 & 16.46896 & 0.00 & 4 & 0.1 \\ \text { 86TC023 } & 57.73371 & 16.46896 & 0.00 & 19 & 0.1 \\ \text { 86TC024 } & 57.62510 & 16.50157 & 0.02 & 4 & 0.1 \\ \text { 86TC026 } & 57.62510 & 16.50157 & 0.28 & 6 & 0.1 \\ \text { 86TC027 } & 57.70885 & 16.52311 & 0.21 & 4 & 0.1 \\ \text { 86TC028 } & 57.75639 & 16.06863 & 0.05 & 4 & 0.1 \\ \text { 86TC029 } & 57.64516 & 16.08843 & 0.02 & 4 & 0.1 \\ \text { 86TC030 } & 57.59610 & 15.97286 & 0.02 & 4 & 0.1\end{array}$




\begin{tabular}{llllrl} 
Sample & \multicolumn{2}{l}{ Location in UTMS } & $\mathrm{Hg}$ & $\mathrm{Ag}$ & $\mathrm{Au}$ \\
& & & & & \\
86TC031 & 57.59610 & 15.97286 & 0.08 & 4 & 0.2 \\
86TC032 & 57.46687 & 15.92133 & 0.02 & 4 & 0.1 \\
86TC033 & 57.09334 & 15.74043 & 0.00 & 11 & 0.1 \\
86TC034 & 57.18968 & 15.76759 & 0.07 & 7 & 0.1 \\
86TC035 & 57.07653 & 15.88913 & 0.00 & 540 & 4.2 \\
86TC036 & 57.07653 & 15.88913 & 0.00 & 12 & 0.1 \\
86TC037 & 57.04295 & 15.88345 & 0.00 & 330 & 0.4 \\
86TC038 & 57.07301 & 15.85370 & 0.00 & 12 & 0.1 \\
86TC039 & 57.07301 & 15.85370 & 0.00 & 53 & 0.3 \\
86TC040 & 57.07301 & 15.85370 & 0.02 & 4 & 0.1 \\
86TC041 & 57.07301 & 15.85370 & 0.00 & 410 & 4.2 \\
86TC042 & 56.87668 & 15.74984 & 0.00 & 11 & 0.3 \\
86TC043 & 56.87668 & 15.74984 & 0.00 & 12 & 0.1 \\
86TC044 & 56.83083 & 15.78408 & 0.00 & 39 & 1.3 \\
86TC045 & 57.05473 & 16.03499 & 0.00 & 97 & 0.1 \\
86TC046 & 57.05473 & 16.03499 & 0.07 & 4 & 0.1 \\
86TC047 & 57.05764 & 16.07043 & 0.04 & 4 & 0.1 \\
86TC048 & 57.05764 & 16.07043 & 0.00 & 82 & 0.1 \\
86TC049 & 57.08929 & 16.06879 & 0.07 & 4 & 0.1 \\
86TC050 & 57.08929 & 16.06879 & 0.00 & 37 & 0.1 \\
86TC051 & 57.11113 & 16.01912 & 0.00 & 56 & 0.1 \\
86TC052 & 56.91626 & 15.94408 & 0.00 & 35 & 0.3 \\
86TC053 & 56.74150 & 16.16570 & 0.05 & 4 & 0.1 \\
86TC054 & 56.81601 & 16.13354 & 0.04 & 4 & 0.1 \\
86TC055 & 56.78094 & 15.88015 & 0.59 & 4 & 0.1 \\
86TC056 & 56.79160 & 15.81151 & 0.07 & 4 & 0.1 \\
86TC057 & 56.63972 & 15.75233 & 0.07 & 4 & 0.1 \\
86TC058 & 56.48741 & 15.79713 & 0.00 & 10 & 0.1 \\
86TC059 & 56.38702 & 15.94341 & 0.02 & 4 & 0.1 \\
86TC060 & 56.45138 & 16.01842 & 0.00 & 300 & 0.1 \\
86TC061 & 56.49625 & 16.00254 & 0.00 & 35 & 0.1 \\
86TC062 & 56.43964 & 16.05160 & 0.00 & 790 & 4.7 \\
86TC063 & 56.46096 & 16.09720 & 0.00 & 240 & 1.6 \\
86TC065 & 56.54510 & 15.46424 & 0.02 & 4 & 0.1 \\
86TC066 & 56.54510 & 15.46424 & 0.02 & 4 & 0.1 \\
86TC067 & 57.43844 & 15.54513 & 0.00 & 34 & 0.1 \\
86TC069 & 57.43844 & 15.54513 & 0.00 & 18 & 0.1 \\
86TC072 & 57.53788 & 15.42702 & 0.00 & 73 & 0.1
\end{tabular}




\begin{tabular}{llllrl} 
Sample & \multicolumn{2}{l}{ Location in UTMS } & Hg & $\mathrm{Ag}$ & $\mathrm{Au}$ \\
& & & & & \\
86TC073 & 57.48324 & 15.36524 & 0.00 & 130 & 0.1 \\
86TC074 & 57.48324 & 15.36524 & 0.00 & 14 & 0.1 \\
86TC077 & 56.70910 & 15.42785 & 0.00 & 100 & 0.1 \\
86TC078 & 56.70910 & 15.42785 & 0.02 & 4 & 0.1 \\
86TC079 & 56.52402 & 15.29998 & 0.02 & 4 & 0.1 \\
86TC120 & 56.84490 & 14.46636 & 1.10 & 630 & 2.4 \\
86TC121 & 56.84490 & 14.46636 & 0.05 & 4 & 0.1 \\
86TC122 & 56.84490 & 14.46636 & 0.24 & 55 & 0.4 \\
86TC123 & 56.84490 & 14.46636 & 0.02 & 4 & 0.1 \\
86TC124 & 56.52180 & 14.26813 & 0.02 & 4 & 0.1 \\
86TC125 & 56.52180 & 14.26813 & 0.02 & 4 & 0.1 \\
86TC126 & 56.97579 & 14.32274 & 1.40 & 150 & 1.3 \\
86TC127 & 56.97579 & 14.32274 & 1.80 & 42 & 1.8 \\
86TC128 & 56.97579 & 14.32274 & 0.02 & 4 & 0.1 \\
86TC129 & 56.97579 & 14.32274 & 0.02 & 4 & 0.1 \\
86TC173 & 56.65274 & 15.08544 & 0.19 & 250 & 3.6 \\
86TC174 & 56.65274 & 15.08544 & 0.12 & 28 & 0.5 \\
86TC175 & 56.65274 & 15.08544 & 0.18 & 74 & 0.2 \\
86TC176 & 56.65274 & 15.08544 & 0.03 & 4 & 0.1 \\
86TC177 & 55.49328 & 17.72038 & 0.08 & 69 & 0.1 \\
86TC178A & 55.49328 & 17.72038 & 0.02 & 4 & 0.1 \\
86TC178B & 55.49328 & 17.72038 & 0.03 & 6 & 0.1 \\
86TC179 & 55.49328 & 17.72038 & 0.04 & 10 & 0.1 \\
86TC180 & 56.37786 & 15.12206 & 0.07 & 14 & 0.6 \\
86TC181 & 56.37786 & 15.12206 & 0.14 & 28 & 0.1 \\
86TC183 & 56.31553 & 15.01584 & 0.09 & 25 & 0.1 \\
86TC185 & 56.31553 & 15.01584 & 0.06 & 23 & 0.3 \\
86TC186 & 56.31553 & 15.01584 & 0.48 & 400 & 0.8 \\
86TC187 & 56.31553 & 15.01584 & 0.06 & 91 & 2.0 \\
86TC189 & 56.82428 & 14.29108 & 0.02 & 10 & 0.1 \\
86TC190 & 56.82428 & 14.29108 & 0.04 & 9 & 0.1 \\
86TC191 & 56.82428 & 14.29108 & 0.02 & 4 & 0.1 \\
86TC192 & 56.13137 & 15.06894 & 0.04 & 11 & 0.1 \\
86TC193 & 56.19865 & 15.06875 & 0.09 & 27 & 0.2 \\
86TC194 & 56.30563 & 15.23311 & 0.12 & 4 & 0.1 \\
86TC195 & 56.34013 & 15.30728 & 0.03 & 4 & 0.1 \\
86TC197 & 56.15204 & 15.16629 & 0.07 & 10 & 0.1 \\
86TC199 & 56.15204 & 15.16629 & 0.04 & 7 & 0.1 \\
& & & & &
\end{tabular}




\begin{tabular}{llllrl} 
Sample & \multicolumn{2}{l}{ Location in UTMS } & Hg & Ag & Au \\
& & & & & \\
86TC200 & 56.16404 & 14.98601 & 0.02 & 5 & 0.1 \\
86TC201 & 56.16404 & 14.98601 & 0.03 & 4 & 0.1 \\
86TC202 & 56.16404 & 14.98601 & 0.02 & 4 & 0.1 \\
86TC203 & 56.46878 & 14.67680 & 0.02 & 4 & 0.1 \\
86TC204 & 56.46878 & 14.67680 & 0.04 & 4 & 0.1 \\
86TC205 & 56.48798 & 14.59263 & 0.26 & 100 & 1.4 \\
86TC206 & 56.48798 & 14.59263 & 0.46 & 260 & 3.8 \\
86TC207 & 56.48798 & 14.59263 & 0.36 & 200 & 0.1 \\
86TC210 & 56.68145 & 14.72569 & 0.36 & 75 & 5.2 \\
86TC211 & 56.78996 & 14.52363 & 0.02 & 4 & 0.1 \\
86TC212 & 56.78996 & 14.52363 & 0.02 & 4 & 0.1 \\
86TC213 & 56.78996 & 14.52363 & 0.02 & 4 & 0.1 \\
86TC214 & 56.78996 & 14.52363 & 0.04 & 4 & 0.1 \\
86TC215 & 56.78996 & 14.52363 & 0.02 & 4 & 0.1 \\
86TC216 & 56.86789 & 14.53288 & 0.02 & 4 & 0.1 \\
86TC217 & 56.86789 & 14.53288 & 0.02 & 4 & 0.1 \\
86TC218 & 56.86789 & 14.53288 & 0.02 & 4 & 0.1 \\
86TC219 & 56.86789 & 14.53288 & 0.02 & 4 & 0.1 \\
86TC220 & 56.86789 & 14.53288 & 0.57 & 560 & 5.0 \\
86TC221 & 56.58551 & 14.02079 & 0.07 & 85 & 0.1 \\
86TC222 & 56.58551 & 14.02079 & 0.04 & 5 & 0.1 \\
86TC223 & 56.58551 & 14.02079 & 0.05 & 7 & 0.1 \\
86TC227 & 56.51315 & 14.25845 & 0.03 & 4 & 0.1 \\
86TC228 & 56.50062 & 14.27880 & 0.02 & 4 & 0.7 \\
86TC231 & 56.27155 & 19.74397 & 0.22 & 4 & 0.1 \\
86TC232 & 56.27155 & 19.74397 & 0.22 & 4 & 0.1 \\
86TC233 & 56.27155 & 19.74397 & 0.14 & 4 & 0.1 \\
86TC234 & 56.60468 & 19.66360 & 0.04 & 4 & 0.1 \\
86TC235 & 57.29062 & 19.73678 & 0.17 & 4 & 3.5 \\
86TC236 & 57.29062 & 19.73678 & 0.18 & 4 & 0.1 \\
86TC237 & 57.29062 & 19.73678 & 0.03 & 4 & 0.1 \\
86TC238 & 57.07055 & 19.68032 & 0.04 & 4 & 0.1 \\
86TC239 & 57.18320 & 19.67640 & 0.09 & 12 & 2.5 \\
86TC240 & 58.59597 & 17.00853 & 0.02 & 4 & 0.1 \\
86TC244 & 58.59597 & 17.00853 & 0.04 & 6 & 0.1 \\
86TC248 & 58.60336 & 17.25130 & 0.02 & 4 & 0.1 \\
86TC249 & 58.58083 & 17.26179 & 0.02 & 4 & 0.1 \\
86TC250 & 58.69055 & 17.46190 & 0.03 & 4 & 0.1
\end{tabular}


Sample Location in UTMS $\mathrm{Hg} \quad \mathrm{Ag} \quad \mathrm{Au}$

$\begin{array}{llllrl}\text { 86TC251 } & 58.68994 & 17.46190 & 0.04 & 4 & 0.4 \\ \text { 86TC252 } & 58.81631 & 17.52464 & 0.05 & 4 & 0.1 \\ \text { 86TC253 } & 58.81572 & 17.52709 & 0.02 & 4 & 0.1 \\ \text { 86TC254 } & 58.79939 & 17.47105 & 0.02 & 4 & 0.1 \\ \text { 86TC256 } & 58.80001 & 17.47227 & 0.02 & 4 & 0.1 \\ \text { 86TC262 } & 58.66025 & 17.10212 & 0.02 & 4 & 0.1 \\ \text { 86TC264 } & 58.66025 & 17.10212 & 0.03 & 4 & 0.1 \\ \text { 86TC269 } & 58.66025 & 17.10212 & 0.02 & 4 & 0.1 \\ \text { 86TC270 } & 58.66025 & 17.10212 & 0.02 & 4 & 0.1 \\ \text { 86TC272 } & 58.46814 & 17.22948 & 0.02 & 4 & 0.1 \\ \text { 86TC273 } & 58.46813 & 17.22765 & 0.05 & 4 & 0.1 \\ \text { 86TC275 } & 58.40588 & 17.15967 & 0.02 & 4 & 0.1 \\ \text { 86TC277 } & 58.40650 & 17.16088 & 0.02 & 4 & 0.1 \\ \text { 86TC288 } & 55.69482 & 20.06506 & 0.68 & 4 & 0.1 \\ \text { 86TC288 } & 55.69482 & 20.06506 & 1.12 & 4 & 0.1 \\ \text { 86TC290 } & 55.76050 & 20.15314 & 0.20 & 4 & 0.1 \\ \text { 86TC293 } & 55.66942 & 20.21563 & 0.22 & 4 & 0.1 \\ \text { 86TC294 } & 55.66942 & 20.21563 & 1.40 & 4 & 0.1 \\ \text { 86TC295 } & 55.66942 & 20.21563 & 2.80 & 11 & 0.1 \\ \text { 86TC300 } & 55.64987 & 20.28309 & 0.05 & 4 & 0.1 \\ \text { 86TC304 } & 55.64987 & 20.28309 & 0.06 & 4 & 0.1 \\ \text { 86TC305 } & 55.64987 & 20.28309 & 0.05 & 4 & 0.1 \\ \text { 86TC306 } & 55.64987 & 20.28309 & 0.34 & 4 & 0.1 \\ \text { 86TC307 } & 56.59101 & 20.50562 & 0.10 & 4 & 0.1 \\ \text { 86TC308 } & 55.72208 & 20.32651 & 2.60 & 4 & 0.1 \\ \text { 86TC309 } & 55.54820 & 20.25660 & 0.14 & 4 & 0.1 \\ \text { 86TC310 } & 56.01430 & 20.19204 & 0.05 & 4 & 0.1 \\ \text { 86TC312 } & 56.01430 & 20.19204 & 0.05 & 4 & 0.1 \\ \text { 86TC313 } & 56.01430 & 20.19204 & 0.19 & 4 & 0.1 \\ \text { 86TC317 } & 56.00307 & 20.08147 & 0.63 & 9 & 0.1 \\ \text { 86TC318 } & 55.92008 & 20.07094 & 7.80 & 950 & 1.6 \\ \text { 86TC319 } & 55.90152 & 20.00390 & 0.90 & 130 & 0.3 \\ \text { 86TC320 } & 55.90152 & 20.00390 & 0.04 & 4 & 0.1 \\ \text { 86TC321 } & 55.90152 & 20.00390 & 0.06 & 4 & 0.1 \\ \text { 86TC322 } & 55.93816 & 20.01076 & 0.76 & 4 & 0.1 \\ \text { 86TC325 } & 55.91292 & 19.94564 & 0.92 & 15 & 0.7 \\ \text { 86TC326 } & 55.90844 & 19.83805 & 1.50 & 26 & 0.4 \\ \text { 86TC327 } & 55.90844 & 19.83805 & 1.40 & 49 & 0.5\end{array}$




\begin{tabular}{llllrr} 
Sample & \multicolumn{2}{l}{ Location in UTMS } & Hg & Ag & $\mathrm{Au}$ \\
& & & & & \\
86TC328 & 55.94755 & 19.93846 & 1.60 & 43 & 0.2 \\
86TC330 & 55.93665 & 19.53306 & 0.99 & 61 & 0.1 \\
86TC331 & 56.06059 & 19.59873 & 0.00 & 12 & 0.1 \\
86TC332 & 56.08716 & 19.62652 & 0.00 & 44 & 0.1 \\
86TC333 & 55.92410 & 19.68798 & 0.00 & 4 & 1.1 \\
86TC334 & 55.65571 & 19.84563 & 0.41 & 4 & 0.1 \\
86TC339 & 55.41309 & 19.42431 & 0.46 & 11 & 0.1 \\
86TC341 & 55.41309 & 19.42431 & 0.00 & 4 & 1.0 \\
86TC342 & 55.41309 & 19.42431 & 2.20 & 8 & 1.1 \\
86TC343 & 55.41309 & 19.42431 & 0.59 & 4 & 0.4 \\
86TC345 & 55.85804 & 19.66866 & 0.02 & 85 & 0.1 \\
86TC347 & 55.95354 & 19.26920 & 0.00 & 60 & 0.4 \\
86TC348 & 55.95354 & 19.26920 & 0.00 & 16 & 0.4 \\
86TC353 & 55.64370 & 16.61684 & 0.00 & 5 & 0.2 \\
86TC354 & 55.64370 & 16.61684 & 0.63 & 4 & 0.2 \\
86TC356 & 55.64370 & 16.61684 & 0.02 & 4 & 0.1 \\
86TC357 & 55.64370 & 16.61684 & 0.02 & 4 & 0.1 \\
86TC358 & 55.64370 & 16.61684 & 0.02 & 14 & 0.1 \\
86TC359 & 55.64370 & 16.61684 & 0.80 & 39 & 0.1 \\
86TC360 & 55.79382 & 16.51007 & 0.08 & 6 & 0.1 \\
86TC361 & 55.79382 & 16.51007 & 0.20 & 29 & 0.1 \\
86TC362 & 55.72701 & 16.46169 & 1.76 & 10 & 14.6 \\
86TC363 & 55.72701 & 16.46169 & 1.00 & 49 & 2.2 \\
86TC364 & 55.68521 & 16.24015 & 0.02 & 10 & 2.5 \\
86TC365 & 55.76694 & 16.68081 & 0.40 & 4 & 0.1 \\
86TC366 & 55.84368 & 16.56728 & 0.04 & 4 & 0.1 \\
86TC367 & 55.85898 & 16.67203 & 2.20 & 67 & 0.1 \\
86TC368 & 55.85898 & 16.67203 & 1.20 & 24 & 0.1 \\
86TC369 & 55.86732 & 16.72928 & 0.40 & 60 & 0.3 \\
86TC370 & 56.03443 & 16.75349 & 0.02 & 28 & 0.1 \\
86TC371 & 56.06989 & 16.82435 & 0.02 & 54 & 0.1 \\
86TC372 & 56.06989 & 16.82435 & 0.02 & 36 & 0.4 \\
86TC373 & 56.06989 & 16.82435 & 0.22 & 9 & 2.4 \\
86TC374 & 55.92770 & 16.89680 & 0.40 & 52 & 0.5 \\
86TC375 & 55.98242 & 16.95210 & 0.32 & 4 & 0.2 \\
86TC376 & 56.19794 & 16.88397 & 0.02 & 4 & 0.1 \\
86TC377 & 56.33466 & 16.95017 & 1.20 & 4 & 0.1 \\
86TC378 & 56.33466 & 16.95017 & 0.36 & 4 & 0.1
\end{tabular}




\begin{tabular}{llllrl} 
Sample & \multicolumn{2}{l}{ Location in UTMS } & Hg & Ag & $\mathrm{Au}$ \\
& & & & & \\
86TC379 & 56.20006 & 16.59214 & 0.00 & 510 & 8.8 \\
86TC380 & 56.20006 & 16.59214 & 0.60 & 90 & 1.4 \\
86TC381 & 56.20006 & 16.59214 & 2.80 & 43 & 0.8 \\
86TC382 & 58.72289 & 13.03837 & 3.40 & 22 & 1.3 \\
86TC383 & 58.72289 & 13.03837 & 2.40 & 4 & 0.1 \\
86TC384 & 58.72289 & 13.03837 & 0.80 & 4 & 0.1 \\
86TC385 & 58.72289 & 13.03837 & 4.20 & 4 & 0.1 \\
86TC387 & 58.26831 & 12.98515 & 0.02 & 4 & 0.1 \\
86TC388 & 56.33466 & 16.95017 & 0.40 & 30 & 0.1 \\
86TC389 & 56.33466 & 16.95017 & 0.96 & 7 & 0.1 \\
86TC390 & 56.50237 & 17.19613 & 0.40 & 4 & 0.1 \\
86TC391 & 56.70082 & 17.00968 & 0.02 & 10 & 0.1 \\
86TC392 & 56.76633 & 16.95186 & 0.16 & 4 & 0.1 \\
86TC393 & 56.81460 & 16.95354 & 0.20 & 4 & 0.1 \\
86TC394 & 56.60400 & 16.95566 & 0.32 & 4 & 0.1 \\
86TC395 & 56.64492 & 16.64857 & 0.00 & 230 & 4.7 \\
86TC396 & 56.64492 & 16.64857 & 0.00 & 180 & 0.7 \\
86TC397 & 56.64492 & 16.64857 & 0.00 & 200 & 1.7 \\
86TC398 & 56.32413 & 16.90760 & 0.00 & 350 & 8.7 \\
86TC399 & 56.07283 & 16.57890 & 0.00 & 71 & 2.9 \\
86TC400 & 56.15356 & 16.51779 & 0.00 & 140 & 0.3 \\
86TC401 & 56.15925 & 16.46032 & 0.00 & 51 & 4.8 \\
86TC403 & 56.19166 & 16.42929 & 0.00 & 1000 & 3.3 \\
86TC404 & 56.19166 & 16.42929 & 0.00 & 61 & 1.8 \\
86TC405 & 56.19166 & 16.42929 & 0.00 & 95 & 1.6 \\
86TC405A & 56.19166 & 16.42929 & 0 & 64 & 1 \\
86TC406 & 55.94325 & 17.18652 & 0.02 & 4 & 0.1 \\
86TC407 & 55.86116 & 17.20492 & 1.60 & 27 & 0.7 \\
86TC408 & 55.86116 & 17.20492 & 2.40 & 6 & 0.1 \\
86TC409 & 55.86116 & 17.20492 & 0.80 & 5 & 0.2 \\
86TC410 & 55.86116 & 17.20492 & 1.20 & 6 & 0.9 \\
86TC411 & 55.86116 & 17.20492 & 0.60 & 4 & 0.1 \\
86TC412 & 56.04926 & 17.10548 & 1.28 & 4 & 2.6 \\
86TC413 & 55.35197 & 17.03353 & 0.08 & 4 & 0.1 \\
86TC414 & 55.35197 & 17.03353 & 0.20 & 4 & 0.1 \\
86TC416 & 54.71426 & 16.50279 & 0.02 & 4 & 0.1 \\
86TC417 & 54.71426 & 16.50279 & 0.04 & 4 & 0.1 \\
86TC418 & 54.71426 & 16.50279 & 0.02 & 4 & 0.1
\end{tabular}


Sample Location in UTMS $\mathrm{Hg} \quad \mathrm{Ag} \quad \mathrm{Au}$

$\begin{array}{lrrrrr}\text { 86TC419 } & 55.09634 & 16.80439 & 0.04 & 4 & 0.1 \\ \text { 86TC420 } & 55.20032 & 16.78076 & 0.04 & 4 & 0.1 \\ \text { 86TC421 } & 55.66394 & 16.93029 & 0.44 & 4 & 0.1 \\ \text { 86TC422 } & 55.66394 & 16.93029 & 0.02 & 15 & 0.1 \\ \text { 86TC423 } & 55.75803 & 17.07103 & 1.00 & 27 & 0.3 \\ \text { 86TC424 } & 55.75803 & 17.07103 & 0.00 & 170 & 1.2 \\ \text { 86TC425 } & 55.80293 & 17.12404 & 0.20 & 19 & 1.4\end{array}$

Note: The limit of detection for mercury is $0.02 \mathrm{ppm}$, for silver is 3 and 4 $\mathrm{ppm}$ depending on the sample set. and for gold is $0.1 \mathrm{ppm}$. 
TABLE 1: ROCK CHEMISTRY OF THE ADELAIDE MINING DISTRICT, NEUADA: MERCURY, SILUER AND GOLD -COOKRO AND THEODORE, 1989

SORTED BY SAMPLE TYPE

QUALIFIER CODES: L=LESS THAN THE LIMIT OF DETECTION $H=$ INTERFERENCE

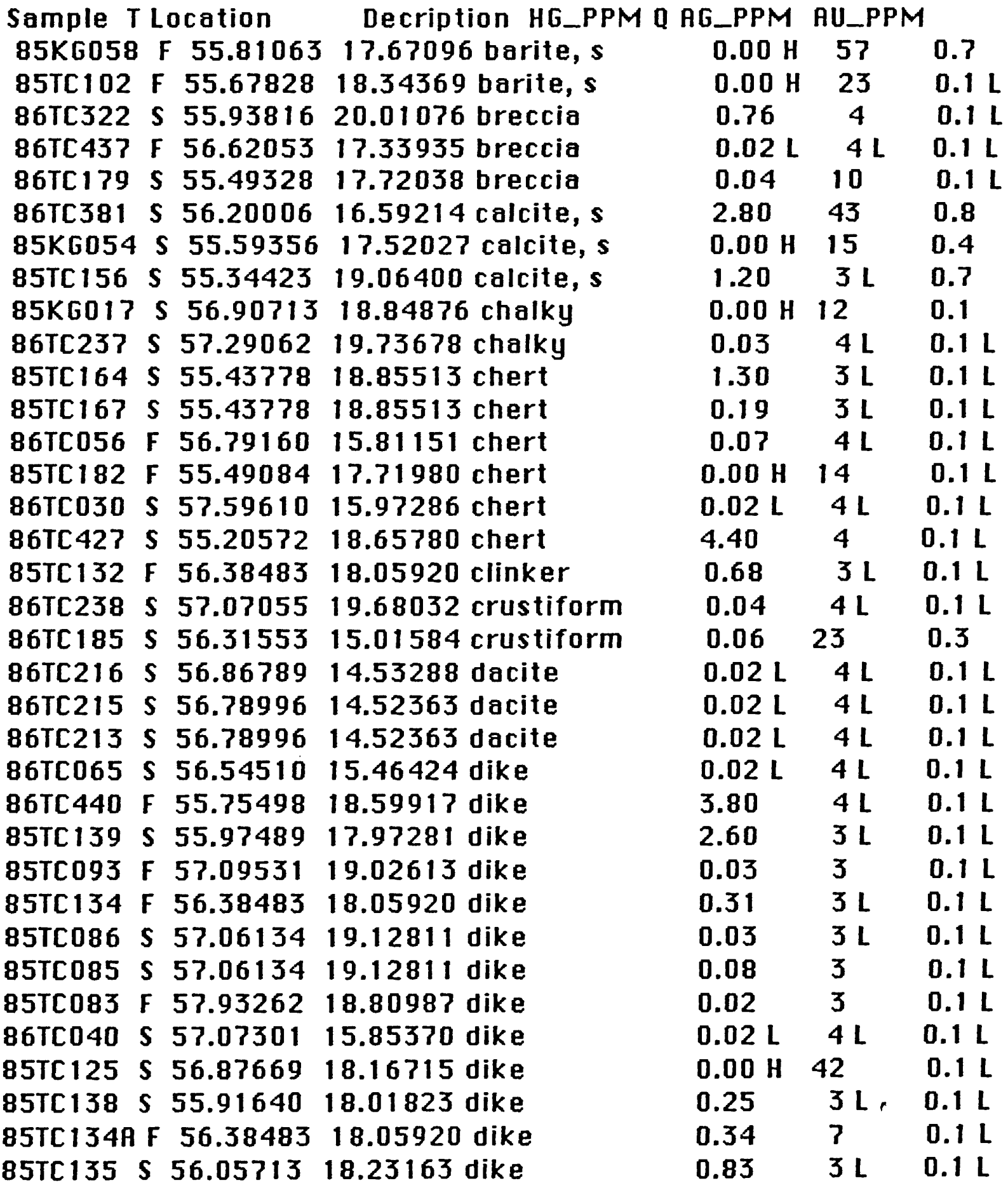




\begin{tabular}{|c|c|c|c|c|c|c|}
\hline $35 T C 137$ & $\mathbf{S}$ & 55.91640 & 18.01823 dike & 0.62 & $3 \mathrm{~L}$ & $0.1 \mathrm{~L}$ \\
\hline 85KG045 & $\mathbf{S}$ & 54.78681 & 17.05312 dike & 0.17 & $3 \mathbf{L}$ & $0.1 \mathrm{~L}$ \\
\hline $85 T C 090$ & $s$ & 57.05112 & 19.09276 dike & $0.00 \mathrm{H}$ & 27 & 0.1 \\
\hline $85 T C 087$ & $\mathbf{S}$ & 57.06134 & 19.12811 dike & 0.12 & 3 & $0.1 \mathrm{~L}$ \\
\hline $5 K G 021$ & $\mathrm{r}$ & 56.38609 & 18.06224 dike & 0.74 & 3 & $0.1 \mathrm{~L}$ \\
\hline $6 T C 375$ & $\mathbf{F}$ & 55.98242 & 16.95210 dike & 0.32 & $4 \mathrm{~L}$ & 0.2 \\
\hline $6 T C 256$ & $\mathbf{S}$ & 58.80001 & 17.47227 dike & $0.02 \mathrm{~L}$ & $4 \mathrm{~L}$ & 0.1 \\
\hline $15 K G 002$ & $\mathbf{S}$ & .57119 & 17.61918 dolomite & 0.06 & $3 L$ & $0.1 \mathrm{~L}$ \\
\hline $86 T C 347$ & $\mathbf{S}$ & 55.95354 & 19.26920 dolomite & $0.00 \mathrm{H}$ & 60 & 0.4 \\
\hline 86TC334 & $s$ & 55.65571 & 19.84563 dolomite & 0.41 & $4 \mathrm{~L}$ & $0.1 \mathrm{~L}^{\circ}$ \\
\hline $86 T C 343$ & $S$ & 55.41309 & 19.42431 dolomite & 0.59 & $4 L$ & 0.4 \\
\hline $86 T C 429$ & $\mathbf{S}$ & 56.80989 & 19.08591 dolomite & 0.04 & $4 L$ & $0.1 \mathrm{~L}$ \\
\hline $85 K G 044$ & $\mathbf{F}$ & 55.42118 & 18.68918 dolomite & 3.30 & $3 \mathrm{~L}$ & 0.3 \\
\hline $86 T C 420$ & $s$ & 55.20032 & 16.78076 gabbro? & 0.04 & $4 \mathrm{~L}$ & 0 \\
\hline $86 T C 367$ & $\mathbf{s}$ & 55.85898 & 16.67203 gossan & 2.20 & 67 & 0.1 \\
\hline $85 T C 091$ & $\mathbf{S}$ & 57.06183 & 19.07366 gossan & $0.00 \mathrm{H}$ & 11 & 0.2 \\
\hline $86 T C 348$ & $\mathbf{S}$ & 55.95354 & 19.26920 gossan & $0.00 \mathrm{H}$ & 16 & 0.4 \\
\hline $85 T C 147$ & $\mathbf{S}$ & 55.34423 & 19.06400 gossan & 0.44 & $3 \mathrm{~L}$ & $0.1 \mathrm{~L}$ \\
\hline $86 T C 353$ & $\mathbf{S}$ & 55.64370 & 16.61684 gossan & $0.00 \mathrm{H}$ & 5 & 0.2 \\
\hline $86 T C 364$ & $\mathbf{S}$ & 55.68521 & 16.24015 gossan & $0.02 \mathrm{~L}$ & 10 & 2.5 \\
\hline 86TC399 & $\mathbf{S}$ & 56.07283 & 16.57890 gossan & $0.00 \mathrm{H}$ & 71 & 2.9 \\
\hline $86 T C 368$ & $\mathbf{S}$ & 55.85898 & 16.67203 gossan & 1.20 & 24 & 0.1 \\
\hline $85 T C 095$ & $\mathbf{F}$ & 57.08729 & 19.01889 gossan & 0.32 & 8 & 0.6 \\
\hline $86 T C 378$ & $\mathbf{F}$ & 56.33466 & 16.95017 gossan & 0.36 & $4 \mathrm{~L}$ & 0.1 \\
\hline $86 \mathrm{TC} 410$ & $\mathbf{F}$ & 55.86116 & 17.20492 gossan & 1.20 & 6 & 0.9 \\
\hline $86 \mathrm{TC} 380$ & $\mathbf{F}$ & 56.20006 & 16.59214 gossan & 0.60 & 90 & 1.4 \\
\hline $85 T C 092$ & $S$ & 57.06183 & 19.07366 gossan & 0.05 & 4 & $0.1 \mathrm{~L}$ \\
\hline $85 T C 128$ & $\mathbf{S}$ & 56.87669 & 18.16715 gossan & 0.12 & $3 \mathrm{~L}$ & 0.3 \\
\hline $86 T C 385$ & $\mathbf{F}$ & 58.72289 & 13.03837 gossan & 4.20 & $4 \mathrm{~L}$ & $0.1 \mathrm{~L}$ \\
\hline $86 T C 397$ & $\mathbf{S}$ & 56.64492 & 16.64857 gossan & $0.00 \mathrm{H}$ & 200 & 1.7 \\
\hline $35 T C 172$ & $\mathbf{F}$ & 55.04788 & 17.53353 gossan & 0.46 & 5 & 0.1 \\
\hline $6 T C 197$ & $\mathbf{S}$ & 56.12871 & 15.22770 gossan & 0.10 & 10 & $0.1 \mathrm{~L}$ \\
\hline $6 \mathrm{TC} 173$ & $\mathbf{S}$ & 56.65274 & 15.08544 gossan & 0.19 & 250 & 3.6 \\
\hline $6 \mathrm{TC} 221$ & $\mathbf{S}$ & 56.58551 & 14.02079 gossan & 0.07 & 85 & $0.1 \mathrm{~L}$ \\
\hline $6 T C 227$ & $S$ & 56.51315 & 14.25845 gossan & 0.03 & $4 L$ & $0.1 \mathrm{~L}$ \\
\hline $5 T C 126$ & $S$ & 56.97579 & 14.32274 gossan & 1.40 & $150^{\circ}$ & 1.3 \\
\hline $6 \mathrm{TC} 127$ & $\mathbf{S}$ & 56.97579 & 14.32274 gossan & 1.80 & 42 & 1.8 \\
\hline $6 T C 239$ & $\mathbf{S}$ & 57.18320 & 19.67640 gossan & 0.09 & 12 & 2.5 \\
\hline $86 \mathrm{TC} 061$ & $S$ & 56.49625 & 16.00254 gossan & $0.00 \mathrm{H}$ & 35 & $0.1 \mathrm{~L}$ \\
\hline $86 T C 181$ & $S$ & 56.37786 & 15.12206 gossan & 0.14 & 28 & $0.1 \mathrm{~L}$ \\
\hline
\end{tabular}




\begin{tabular}{|c|c|c|c|c|c|}
\hline $6 \mathrm{TC} 204$ & S 56.46878 & & 0.04 & $4 \mathrm{~L}$ & o. \\
\hline $86 T C 198$ & S 56.15204 & 15.16629 gossan & 0.07 & 10 & \\
\hline $86 T C 063$ & S 56.46096 & 16.09720 gossan & $0.00 \mathrm{H}$ & 240 & 1.6 \\
\hline $85 T C 184$ & S 55.59619 & 17.80772 gossan & $0.00 \mathrm{H}$ & 27 & 0.3 \\
\hline $86 T C 036$ & S 57.07653 & 15.88913 gossan & $0.00 \mathrm{H}$ & 12 & \\
\hline $86 T C 037$ & S 57.04295 & 15.88345 gossan & $0.00 \mathrm{H}$ & 330 & 0.4 \\
\hline $86 T C 186$ & S 56.31553 & 15.01584 gossan & 0.48 & 400 & 0.8 \\
\hline $86 T C 034$ & S 57.18968 & 15.76759 gossan & 0.07 & $?$ & 0.1 \\
\hline $86 T C 035$ & S 57.07653 & 15.88913 gossan & $0.00 \mathrm{H}$ & 540 & 4.2 \\
\hline $85 T C 185$ & S 55.58628 & 17.65922 gossan & $0.00 \mathrm{H}$ & 21 & 0.1 \\
\hline $86 T C 060$ & S 56.45138 & 16.01842 gossan & $0.00 \mathrm{H}$ & 300 & 0.1 \\
\hline $86 \mathrm{TC} 328$ & S 55.94755 & 19.93846 gossan & 1.60 & 43 & 0.2 \\
\hline $86 T C 052$ & S 56.91626 & 15.94408 gossan & $0.00 \mathrm{H}$ & 35 & 0.3 \\
\hline $86 \mathrm{TC} 318$ & F 55.92008 & 20.07094 gossan & 7.80 & 950 & 1.6 \\
\hline $86 \mathrm{TC} 027$ & S 57.70885 & 16.52311 gossan & 0.21 & $4 \mathrm{~L}$ & 0.1 \\
\hline $86 \mathrm{TC062}$ & $S \quad 56.43964$ & 16.05160 gossan & $0.00 \mathrm{H}$ & 790 & 4.7 \\
\hline $85 T C 075$ & S 57.06349 & 18.83326 gossan & 0.72 & 5 & $0.1 \mathrm{~L}$ \\
\hline $85 K G 015$ & F 57.05797 & 18.46572 gossan & $0.00 \mathrm{H}$ & 13 & 70.0 \\
\hline $85 K G 013$ & S 56.92472 & 18.43320 gossan & 0.04 & $3 \mathrm{~L}$ & $0.1 \mathrm{~L}$ \\
\hline $85 T C 143$ & F 55 . & 19 jasper & 1.00 & $3 \mathrm{~L}$ & $0.1 \mathrm{~L}$ \\
\hline $85 T C 161$ & F 55.43778 & 18.85513 jasperoid & 0.07 & $3 \mathrm{~L}$ & $0.1 \mathrm{~L}$ \\
\hline $86 \mathrm{TC} 310$ & $S 56.01430$ & 20.19204 jasperoid & 0.05 & $4 \mathrm{~L}$ & $0.1 \mathrm{~L}$ \\
\hline $86 T C 015$ & F 58.05893 & 16.62779 jasperoid & $0.02 \mathrm{~L}$ & $4 \mathrm{~L}$ & $0.1 \mathrm{~L}$ \\
\hline $86 T C 294$ & S 55.66942 & 20.21563 jasperoid & 1.40 & $4 \mathrm{~L}$ & $0.1 \mathrm{~L}$ \\
\hline $86 \mathrm{TC006}$ & F 58.15807 & 16.88225 jasperoid & $0.00 \mathrm{H}$ & 17 & $0.1 \mathrm{~L}$ \\
\hline $86 T C 011$ & F 57.97242 & 16.81861 jasperoid & $0.02 \mathrm{~L}$ & $4 \mathrm{~L}$ & 0.1 \\
\hline $86 \operatorname{TC} 312$ & S 56.01430 & 20.19204 jasperoid & 0.05 & $4 \mathrm{~L}$ & $0.1 \mathrm{~L}$ \\
\hline $86 T C 313$ & $S 56.01430$ & 20.19204 jasperoid & 0.19 & $4 \mathrm{~L}$ & $0.1 \mathrm{~L}$ \\
\hline $85 T C 159$ & S 55.43778 & 18.85513 Mn oride & $0.00 \mathrm{H}$ & 26 & $0.1 \mathrm{~L}$ \\
\hline $85 T C 155$ & S 55.34423 & 19.06400 Mn oside & 4.40 & 4 & 0.1 \\
\hline $85 K G 039$ & S 55.52324 & 18.77695 Mn овide & 0.07 & $3 \mathrm{~L}$ & $0.1 \mathrm{~L}$ \\
\hline $86 T C 048$ & S 57.05764 & 16.07043 Mn oride & $0.00 \mathrm{H}$ & 82 & 0.1 \\
\hline $86 T C 050$ & S 57.08929 & $16.06879 \mathrm{Mn}$ oside & $0.00 \mathrm{H}$ & 37 & $0.1 \mathrm{~L}$ \\
\hline $86 T C 026$ & S 57.62510 & 16.50157 Mn oside & 0.28 & 6 & $0.1 \mathrm{~L}$ \\
\hline $86 T C 073$ & S 57.48324 & $15.36524 \mathrm{Mn}$ oside & $0.00 \mathrm{H}$ & 130 & $0.1 \mathrm{~L}$ \\
\hline $86 T C 356$ & S 55.64370 & 16.61684 Mn oside & $0.02 \mathrm{~L}$ & 4 & $0.1 \mathrm{~L}$ \\
\hline $86 T C 333$ & S 55.92410 & 19.68798 Mn oride & $0.00 \mathrm{H}$ & $4 \mathrm{~L}$ & 1.1 \\
\hline $86 T C 023$ & S 57.73371 & $16.46896 \mathrm{Mn}$ oside & $0.00 \mathrm{H}$ & 19 & 0.1 \\
\hline $86 \mathrm{TCO} 21$ & S 57.79901 & 16.56841 Mn oside & 0.36 & $.4 \mathrm{~L}$ & $0.1 \mathrm{~L}$ \\
\hline $86 T C 354$ & S 55.64370 & 16.61684 Mn oside & 0.63 & $4 \mathrm{~L}$ & 0.2 \\
\hline $86 T C 339$ & S 55.41309 & $19.42431 \mathrm{Mn}$ oride & 0.46 & 11 & $0.1 \mathrm{~L}$ \\
\hline
\end{tabular}




\begin{tabular}{|c|c|c|c|c|c|c|}
\hline $86 T C 331$ & $\mathbf{S}$ & 56.06059 & $19.59873 \mathrm{Mn}$ & $0.00 \mathrm{H}$ & 12 & 0.1 \\
\hline $86 T C 438$ & $\mathbf{F}$ & 56.43540 & 17.26531 obsidian & $0.02 \mathrm{~L}$ & $4 \mathrm{~L}$ & $0.1 \mathrm{~L}$ \\
\hline $86 T C 217$ & $\mathbf{S}$ & 56.86789 & 14.53288 phyllitic & 0.02 & $4 \mathrm{~L}$ & $0.1 \mathrm{~L}$ \\
\hline $85 T C 068$ & $\mathbf{S}$ & 57.06367 & 18.71031 phyllitic & 0.03 & $3 \mathrm{~L}$ & $0.1 \mathrm{~L}$ \\
\hline $86 T C 200$ & $\mathbf{F}$ & 56.16404 & 14.98601 phyllitic & 0.02 & 5 & $0.1 \mathrm{~L}$ \\
\hline $85 T C 067$ & $s$ & 57.06367 & 18.71031 phyllitic & 0.04 & $3 \mathrm{~L}$ & $0.1 \mathrm{~L}$ \\
\hline $86 \mathrm{TC} 406$ & $\mathbf{F}$ & 55.94325 & 17.18652 phyllitic & $0.02 \mathrm{~L}$ & $4 \mathrm{~L}$ & $0.1 \mathrm{~L}$ \\
\hline $85 T C 065$ & $\mathbf{S}$ & 57.06367 & 18.71031 phyllitic & 0.34 & $3 \mathrm{~L}$ & $0.1 \mathrm{~L}$ \\
\hline $85 T C 064$ & $\mathbf{F}$ & 58.27589 & 16.94626 phyllitic & 0.07 & 46 & 0.1 \\
\hline $86 T C 366$ & $\mathbf{S}$ & 55.84368 & 16.56728 phyllitic & 0.04 & $4 \mathrm{~L}$ & 0.1 \\
\hline $86 T C 365$ & $\mathbf{S}$ & 55.76694 & 16.68081 phyllitic & 0.40 & $4 i$ & 0.1 \\
\hline $85 K G 024$ & $S$ & 55.93018 & 18.18320 phyllitic & 0.70 & $3 \mathrm{~L}$ & $0.1 \mathrm{~L}$ \\
\hline $86 T C 031$ & $\mathbf{S}$ & 57.59610 & 15.97286 phyllitic & 0.08 & $4 \mathrm{~L}$ & 0.2 \\
\hline $86 T C 029$ & $\mathbf{S}$ & 57.64516 & 16.08843 phyllitic & $0.02 \mathrm{~L}$ & $4 \mathrm{~L}$ & $0.1 \mathrm{~L}$ \\
\hline $86 T C 024$ & $\mathbf{F}$ & 57.62510 & 16.50157 phyllitic & 0.02 & $3 \mathrm{~L}$ & $0.1 \mathrm{~L}$ \\
\hline $86 T C 195$ & $\mathbf{F}$ & 56.34013 & 15.30728 phyllitic & 0.03 & $4 \mathrm{~L}$ & $0.1 \mathrm{~L}$ \\
\hline $85 T C 074$ & $\mathbf{S}$ & 57.06349 & 18.83326 phyllitic & 0.05 & $3 \mathrm{~L}$ & $0.1 \mathrm{~L}$ \\
\hline $85 T C 099$ & $\mathbf{S}$ & 57.17725 & 18.95777 phyllitic & $0.02 \mathrm{~L}$ & $3 \mathrm{~L}$ & $0.1 \mathrm{~L}$ \\
\hline $85 T C 100$ & $\mathbf{S}$ & 57.17725 & 18.95777 phyllitic & $0.02 \mathrm{~L}$ & $3 \mathrm{~L}$ & $0.1 \mathrm{~L}$ \\
\hline $85 T C 098$ & $\mathbf{S}$ & 57.17725 & 18.95777 phyllitic & $0.02 \mathrm{~L}$ & $3 \mathrm{~L}$ & $0.1 \mathrm{~L}$ \\
\hline $85 T C 073$ & $\mathbf{S}$ & 57.06349 & 18.83326 phyllitic & 0.02 & $3 \mathrm{~L}$ & $0.1 \mathrm{~L}$ \\
\hline $85 K G 041$ & $\mathbf{S}$ & 54.80167 & 18.43835 phyllitic & 0.10 & $3 \mathrm{~L}$ & 0.1 \\
\hline $86 T C 079$ & $S$ & 56.52402 & 15.29998 phyllitic & $0.02 \mathrm{~L}$ & $4 \mathrm{~L}$ & $0.1 \mathrm{~L}$ \\
\hline $85 T C 124$ & $\mathbf{F}$ & 56.97281 & 17.97383 phyllitic & 0.03 & $3 \mathrm{~L}$ & $0.1 \mathrm{~L}$ \\
\hline $85 K G 027$ & $\mathbf{S}$ & 55.91488 & 18.22316 phyllitic & 0.29 & $3 \mathrm{~L}$ & $0.1 \mathrm{~L}$ \\
\hline $85 T C 126$ & $\mathbf{S}$ & 56.87669 & 18.16715 phyllitic & 0.02 & $3 \mathrm{~L}$ & $0.1 \mathrm{~L}$ \\
\hline $86 T C 201$ & $\mathbf{S}$ & 56.16404 & 14.98601 phyllitic & 0.03 & $4 \mathrm{~L}$ & $0.1 \mathrm{~L}$ \\
\hline $85 T C 071$ & $\mathbf{S}$ & 57.06349 & 18.83326 phyllitic & 0.03 & $3 L$ & $0.1 \mathrm{~L}$ \\
\hline $85 T C 078$ & $\mathbf{S}$ & 57.30080 & 18.67540 phyllitic & 0.03 & $3 L$ & $0.1 \mathrm{~L}$ \\
\hline $86 T C 409$ & $\mathbf{S}$ & 55.86116 & 17.20492 phyllitic & 0.80 & 5 & 0.2 \\
\hline $85 T C 080$ & $\mathbf{F}$ & 57.39147 & 18.66748 phyllitic & 0.02 & $3 \mathrm{~L}$ & $0.1 \mathrm{~L}$ \\
\hline $85 T C 081$ & $\mathbf{S}$ & 57.90168 & 18.88911 phyllitic & 0.05 & $3 \mathrm{~L}$ & 10.0 \\
\hline $86 \mathrm{TC} 202$ & $\mathbf{s}$ & 56.16404 & 14.98601 phyllitic & 0.02 & $4 \mathrm{~L}$ & $0.1 \mathrm{~L}$ \\
\hline $85 T C 069$ & $\mathbf{S}$ & 57.08014 & 18.75719 phyllitic & 0.09 & $3 \mathrm{~L}$ & $0.1 \mathrm{~L}$ \\
\hline $85 K G 037$ & $\mathbf{S}$ & 55.40460 & 18.88004 phyllitic & 0.03 & $3 \mathrm{~L}$ & $0.1 \mathrm{~L}$ \\
\hline $86 T C 434$ & $\mathbf{F}$ & 56.13516 & $18.90110 \mathrm{qtz}$ monz & $0.02 \mathrm{~L}$ & $4 \mathrm{~L}$ & $0.1 \mathrm{~L}$ \\
\hline $86 \mathrm{TC} 431$ & $\mathbf{F}$ & 57.05112 & $19.09276 \mathrm{qtz}$ monz & 0.10 & $4 \mathrm{~L}$ & $0.1 \mathrm{~L}$ \\
\hline $86 T C 178 B$ & & 49328 & 17.72038 & 0.03 & 6 & $0.1 \mathrm{~L}$ \\
\hline $86 \mathrm{TC} 178 \mathrm{~A}$ & & 55.49328 & 17.72038 quartz & 0.02 & 4 & $0.1 \mathrm{~L}$ \\
\hline $86 T C 032$ & & 57.46687 & 15.92133 quartz & $0.02 \mathrm{~L}$ & $4 \mathrm{~L}$ & $0.1 \mathrm{~L}$ \\
\hline
\end{tabular}




\begin{abstract}
86TC183 S 56.3155315 .01584 quartz
$85 K G 042$ S 54.9014518 .47435 quartz 86 TC176 S 56.6527415 .08544 quartz 86TC128 S 56.9757914 .32274 quartz 86TC206 S 56.4879814 .59263 quartz 86TC222 S 56.5855114 .02079 quartz 85KG055 S 55.5216017 .42335 quartz 86TC223 S 56.5855114 .02079 quartz 85KG052 S 55.5935617 .52027 quartz 86TC069 F 57.4384415 .54513 quartz 86 TC220 S 56.8678914 .53288 quartz 86TC231 S 56.2715519 .74397 quartz $86 T C 218$ S 56.8678914 .53288 quartz 86 TC211 S 56.7899614 .52363 quartz 86TC067 S 57.4384415 .54513 quartz 86TC254 F 58.79939 17.47105 quartz 85KG031 F 55.3988519 .13334 quartz $86 T C 074$ S 57.4832415 .36524 quartz 86TC038 S 57.0730115 .85370 quartz 86TC039 S 57.0730115 .85370 quartz 85KG030 F 55.3988519 .13334 quartz 86TC205 S 56.4879814 .59263 quartz 86 TC044 S 56.8308315 .78408 quartz $86 T C 043$ S 56.8766815 .74984 quartz 86 TC072 S 57.5378815 .42702 quartz 86TC042 S 56.8766815 .74984 quartz 85KG049 F 55.0478817 .53353 quartz 86TC430 S 57.0511219 .09276 quartz 85TC141 S 55.4837118 .41719 quartz $85 T C 163$ S 55.4377818 .85513 quartz 86TC379 F 56.2000616 .59214 quartz $86 T C 387$ S 58.2683112 .98515 quartz 86 TC395 S 56.6449216 .64857 quartz $85 T C 120$ S 57.1623417 .93342 quartz 86 TC370 F 56.0344316 .75349 quartz 85TC142 S 55.4837118 .41719 quartz 86TC371 F 56.0698916 .82435 quartz $85 T C 070$ S 57.0801418 .75719 quartz $85 T C 072$ S 57.0634918 .83326 quartz 86TC412 F 56.0492617 .10548 quartz 86TC422 F 55.6639416 .93029 quartz 85TC130 F 56.9071318 .84876 quartz $85 T C 129$ F 56.9071318 .84876 quartz
\end{abstract}

\begin{tabular}{|c|c|c|}
\hline 0.09 & 25 & 0.1 \\
\hline 0.76 & 31 & $0.1 \mathrm{~L}$ \\
\hline 0.03 & $4 L$ & $0.1 \mathrm{~L}$ \\
\hline 0.02 & $4 \mathrm{~L}$ & 0.1 \\
\hline 0.46 & 260 & 3.8 \\
\hline 0.04 & 5 & $0.1 \mathrm{~L}$ \\
\hline $0.00 \mathrm{H}$ & 15 & 0.9 \\
\hline 0.05 & 7 & $0.1 \mathrm{~L}$ \\
\hline $0.00 \mathrm{H}$ & 97 & 3.5 \\
\hline $0.00 \mathrm{H}$ & 18 & $0.1 \mathrm{~L}$ \\
\hline 0.57 & 560 & 5.0 \\
\hline 0.22 & $4 L$ & $0.1 \mathrm{~L}$ \\
\hline 0.02 & $4 L$ & $0.1 \mathrm{~L}$ \\
\hline $0.02 \mathrm{~L}$ & $4 L$ & $0.1 \mathrm{~L}$ \\
\hline $0.00 \mathrm{H}$ & 34 & $0.1 \mathrm{~L}$ \\
\hline 0.02 & $4 L$ & $0.1 \mathrm{~L}$ \\
\hline $0.00 \mathrm{H}$ & 19 & 0.1 \\
\hline $0.00 \mathrm{H}$ & 14 & $0.1 \mathrm{~L}$ \\
\hline $0.00 \mathrm{H}$ & 12 & 0.1 \\
\hline $0.00 \mathrm{H}$ & 53 & 0.3 \\
\hline 2.00 & 4 & $0.1 \mathrm{~L}$ \\
\hline 0.26 & 100 & 1.4 \\
\hline $0.00 \mathrm{H}$ & 39 & 1.3 \\
\hline $0.00 \mathrm{H}$ & 12 & 0.1 \\
\hline $0.00 \mathrm{H}$ & 73 & $0.1 \mathrm{~L}$ \\
\hline $0.00 \mathrm{H}$ & 11 & 0.3 \\
\hline 0.34 & $3 \mathrm{~L}$ & $0.1 \mathrm{~L}$ \\
\hline 0.04 & $4 L$ & $0.1 \mathrm{~L}$ \\
\hline $0.00 \mathrm{H}$ & 9 & 0.3 \\
\hline 8.40 & $3 \mathrm{~L}$ & 0.4 \\
\hline $0.00 \mathrm{H}$ & 510 & 8.8 \\
\hline $0.02 \mathrm{~L}$ & $4 L$ & $0.1 \mathrm{~L}$ \\
\hline $0.00 \mathrm{H}$ & 230 & 4.7 \\
\hline 0.07 & $3 \mathbf{L}$ & $0.1 \mathrm{~L}$ \\
\hline $0.02 \mathrm{~L}$ & 28 & 0.1 \\
\hline $0.00 \mathrm{H}$ & 17 & 0.8 \\
\hline $0.02 \mathrm{~L}$ & 54 & 0.1 \\
\hline 0.06 & $3 \mathbf{L}$ & $0.1 \mathrm{~L}$ \\
\hline 0.07 & 3 & $0.1 \mathrm{~L}$ \\
\hline 1.28 & $4 L$ & 2.6 \\
\hline $0.02 \mathrm{~L}$ & 1,5 & $0.1 \mathrm{~L}$ \\
\hline $0.00 \mathrm{H}$ & 14 & 0.1 \\
\hline $0.00 \mathrm{H}$ & 110 & 0.3 \\
\hline
\end{tabular}




\begin{tabular}{|c|c|c|c|c|c|c|}
\hline $86 T C 398$ & $\mathbf{S}$ & 56.32413 & 16.90760 quartz & $0.00 \mathrm{H}$ & 350 & 8.7 \\
\hline $85 T C 127$ & $\mathbf{F}$ & 56.87669 & 18.16715 quartz & 0.06 & $3 \mathrm{~L}$ & $0.1 \mathrm{~L}$ \\
\hline $86 T C 360$ & $\mathbf{F}$ & 55.79382 & 16.51007 quartz & 0.08 & 6 & 0.1 \\
\hline $86 T C 424$ & $\mathbf{F}$ & 55.75803 & 17.07103 quartz & $0.00 \mathrm{H}$ & 170 & 1.2 \\
\hline $85 T C 082$ & $S$ & 57.90168 & 18.88911 quartz & $0.02 \mathrm{~L}$ & $3 \mathrm{~L}$ & 0.1 \\
\hline $86 T C 341$ & $S$ & 55.41309 & 19.42431 quartz & $0.00 \mathrm{H}$ & 4 & 1.0 \\
\hline $85 K G 022$ & S & 56.02438 & 18.16545 quartz & 0.89 & $3 L$ & $0.1 \mathrm{~L}$ \\
\hline $85 K G 020$ & $\mathbf{F}$ & 56.38609 & 18.06224 quartz & 0.40 & $3 \mathrm{~L}$ & $0.1 \mathrm{~L}$ \\
\hline $86 T C 008$ & $\mathbf{S}$ & 58.16114 & 16.88467 quartz & 0.04 & $4 \mathrm{~L}$ & $0.1 \mathrm{~L}$ \\
\hline $86 \mathrm{TC} 358$ & $\mathbf{F}$ & 55.64370 & 16.61684 quartz & $0.02 \mathrm{~L}$ & 14 & 0.1 \\
\hline $86 T C 359$ & $S$ & 55.64370 & 16.61684 quartz & 0.80 & 39 & 0.1 \\
\hline $86 T C 369$ & $\mathbf{S}$ & 55.86732 & 16.72928 quartz & 0.40 & 60 & 0.3 \\
\hline $86 T C 361$ & $\mathbf{S}$ & 55.79382 & 16.51007 quartz & 0.20 & 29 & 0.1 \\
\hline $85 K G 016$ & $S$ & 57.05797 & 18.46572 quartz & 0.02 & $3 \mathrm{~L}$ & 0.3 \\
\hline $85 K G 018$ & $\mathbf{F}$ & 56.90713 & 18.84876 quartz & $0.00 \mathrm{H}$ & 24 & $0.1 \mathrm{~L}$ \\
\hline $85 T C 177$ & $\mathbf{S}$ & 55.49084 & 17.71980 quartz & $0.00 \mathrm{H}$ & 10 & 0.1 \\
\hline $86 T C 363$ & $\mathbf{S}$ & 55.72701 & 16.46169 quartz & 1.00 & 49 & 2.2 \\
\hline $85 K G 005$ & $\mathbf{S}$ & 57.16234 & 17.93342 quartzite & 0.21 & $3 \mathrm{~L}$ & $0.1 \mathrm{~L}$ \\
\hline $85 K G 003$ & $\mathbf{S}$ & 57.17606 & 17.95710 quartzite & 0.24 & & $0.1 \mathrm{~L}$ \\
\hline $86 \mathrm{TC} 177$ & $\mathbf{S}$ & 55.49328 & 17.72038 quartzite & 0.08 & 69 & $0.1 \mathrm{~L}$ \\
\hline $86 T C 193$ & $\mathbf{F}$ & 56.19865 & 15.06875 quartzite & 0.09 & $4 L$ & $0.1 \mathrm{~L}$ \\
\hline $86 T C 432$ & $\mathbf{F}$ & 57.05797 & 18.46572 quartzite & $0.02 \mathrm{~L}$ & $4 \mathrm{~L}$ & $0.1 \mathrm{~L}$ \\
\hline $86 T C 175$ & $\mathbf{S}$ & 56.65274 & 15.08544 quartzite & 0.18 & 74 & 0.2 \\
\hline $86 T C 428$ & $\mathbf{S}$ & 55.30969 & 18.80510 quartzite & $0.02 \mathrm{~L}$ & $4 \mathrm{~L}$ & $0.1 \mathrm{~L}$ \\
\hline $86 T C 320$ & $S$ & 55.90152 & 20.00390 quartzite & 0.04 & $4 L$ & $0.1 \mathrm{~L}$ \\
\hline $86 T C 232$ & $S$ & 56.27155 & 19.74397 quartzite & 0.22 & $4 \mathrm{~L}$ & $0.1 \mathrm{~L}$ \\
\hline $86 T C 394$ & $\mathbf{S}$ & 56.60400 & 16.95566 quartzite & 0.32 & $4 \mathrm{~L}$ & $0.1 \mathrm{~L}$ \\
\hline $86 \mathrm{TC} 321$ & $S$ & 55.90152 & 20.00390 quartzite & 0.06 & $4 \mathrm{~L}$ & 0.1 \\
\hline $86 T C 236$ & $\mathbf{S}$ & 57.29062 & 19.73678 quartzite & 0.18 & $4 \mathrm{~L}$ & $0.1 \mathrm{~L}$ \\
\hline $86 T C 235$ & $\mathbf{S}$ & 57.29062 & 19.73678 quartzite & 0.17 & $4 \mathrm{~L}$ & 3.5 \\
\hline $85 K G 006$ & $\mathbf{S}$ & 57.16234 & 17.93342 quartzite & 0.21 & $3 L$ & $0.1 \mathrm{~L}$ \\
\hline $86 T C 390$ & $\mathbf{S}$ & 56.50237 & 17.19613 quartzite & 0.40 & $4 \mathrm{~L}$ & $0.1 \mathrm{~L}$ \\
\hline $85 K G 007$ & $\mathbf{S}$ & 56.98806 & 17.88432 quartzite & 0.07 & $3 \mathrm{~L}$ & $0.1 \mathrm{~L}$ \\
\hline $86 T C 207$ & $\mathbf{S}$ & 56.48798 & 14.59263 quartzite & 0.36 & 200 & $0.1 \mathrm{~L}$ \\
\hline $86 T C 407$ & $\mathbf{F}$ & 55.86116 & 17.20492 quartzite & 1.60 & 27 & 0.7 \\
\hline $86 T C 408$ & $\mathbf{F}$ & 55.86116 & 17.20492 quartzite & 2.40 & 6 & 0.1 \\
\hline $86 T C 174$ & $\mathbf{S}$ & 56.65274 & 15.08544 quartzite & 0.12 & 28 & 0.5 \\
\hline $85 T C 089$ & $\mathbf{S}$ & 57.05112 & 19.09276 quartzite & $0.00 \mathrm{H}$ & 8 & $0.1 \mathrm{~L}$ \\
\hline $86 T \cos 7$ & $\mathbf{F}$ & 56.70910 & 15.42785 quartzite & $0.00 \mathrm{H}$ & 10 & $0.1 \mathrm{~L}$ \\
\hline $85 K G 001$ & S & 57.57119 & 17.61918 quartzite & 0.06 & $.3 \mathrm{~L}$ & $0.1 \mathrm{~L}$ \\
\hline $85 T C 117$ & $\mathbf{F}$ & 57.59252 & 17.50710 quartzite & $0.02 \mathrm{~L}$ & $3 \mathrm{~L}$ & $0.1 \mathrm{~L}$ \\
\hline $85 T C 123$ & $\mathbf{S}$ & 56.97281 & 17.97383 quartzite & 0.03 & $3 \mathrm{~L}$ & $0.1 \mathrm{~L}$ \\
\hline
\end{tabular}




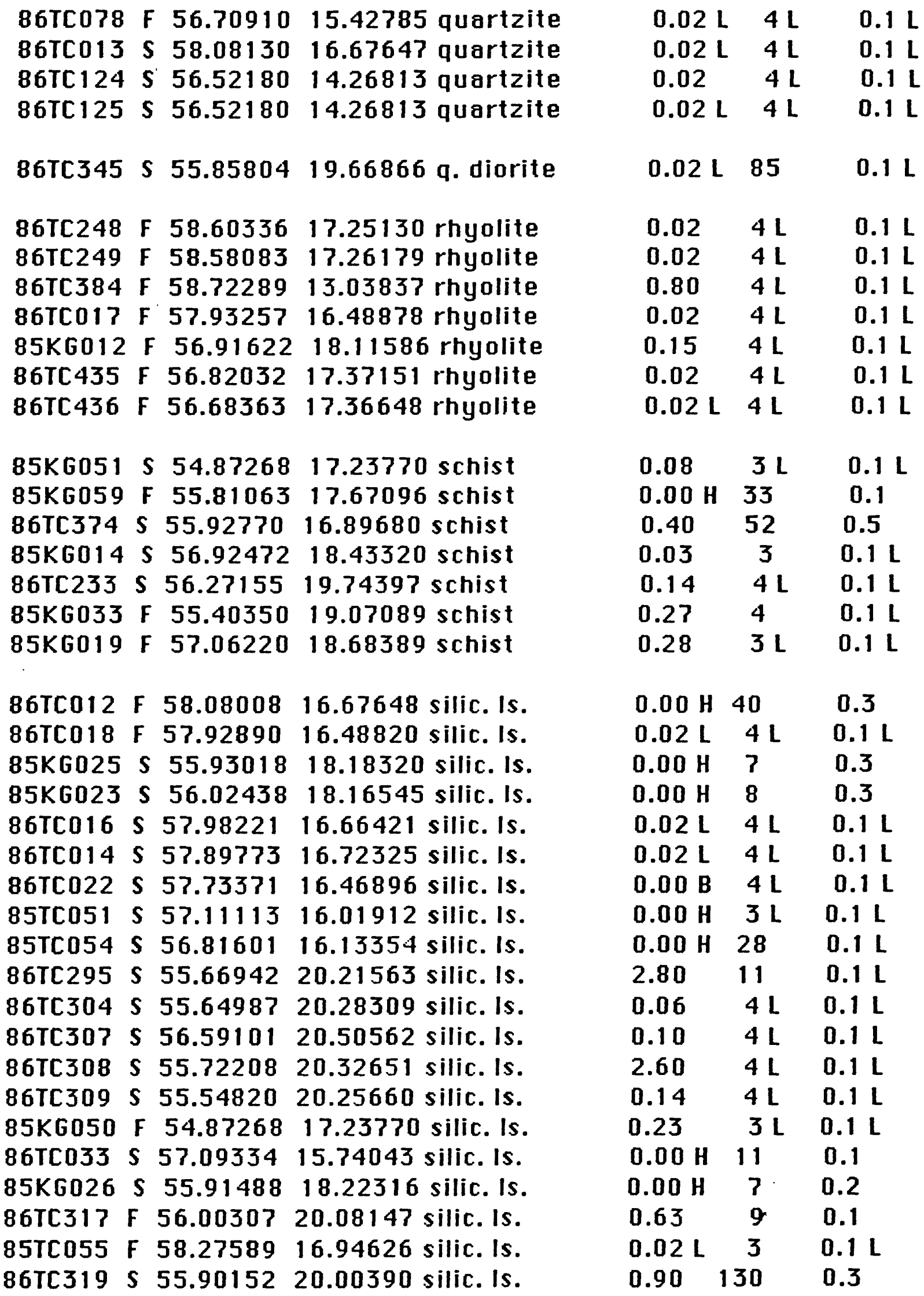




\begin{tabular}{|c|c|c|c|c|c|c|}
\hline $85 T C 056$ & $\mathbf{F}$ & 58.27589 & 16.94626 silic. Is. & $0.02 \mathrm{~L}$ & 3 & $0.1 \mathrm{~L}$ \\
\hline $86 T C 019$ & $\mathbf{F}$ & 57.92793 & 16.44184 silic. Is. & $0.02 \mathrm{~L}$ & $4 \mathrm{~L}$ & $0.1 \mathrm{~L}$ \\
\hline $86 T C 010$ & $\mathbf{S}$ & 57.97242 & 16.81861 silic. Is. & 0.03 & $4 L$ & $0.1 \mathrm{~L}$ \\
\hline $86 T C 293$ & $\mathbf{S}$ & 55.66942 & 20.21563 silic. Is. & 0.22 & $4 \mathrm{~L}$ & $0.1 \mathrm{~L}$ \\
\hline $85 K G 004$ & $\mathbf{S}$ & 57.17606 & 17.95710 silic. Is. & 0.17 & $3 \mathrm{~L}$ & $0.1 \mathrm{~L}$ \\
\hline $85 T C 118$ & $S$ & 57.59252 & 17.50710 silic. Is. & 0.03 & $3 \mathrm{~L}$ & $0.1 \mathrm{~L}$ \\
\hline $85 T C 133$ & $S$ & 56.38483 & 18.05920 silic. Is. & 0.11 & $3 \mathrm{~L}$ & $0.1 \mathrm{~L}$ \\
\hline $85 T C 076$ & 5 & 57.12843 & 18.90581 silic. Is. & 0.03 & $3 \mathrm{~L}$ & $0.1 \mathrm{~L}$ \\
\hline $85 T C 131$ & $\mathbf{F}$ & 56.90713 & 18.84876 silic. Is. & $0.00 \mathrm{H}$ & 11 & $0.1 \mathrm{~L}$ \\
\hline $85 T C 122$ & $S$ & 56.97281 & 17.97383 silic. Is. & 0.03 & $3 \mathrm{~L}$ & $0.1 \mathrm{~L}$ \\
\hline $85 T C 149$ & $\mathbf{s}$ & 55.34423 & 19.06400 silic. Is. & $0.00 \mathrm{H}$ & 8 & $0.1 \mathrm{~L}$ \\
\hline $85 T C 121$ & $\mathbf{S}$ & 56.97013 & 17.91086 silic. Is. & 0.03 & $3 L$ & $0.1 \mathrm{~L}$ \\
\hline $85 K G 009$ & $\mathbf{S}$ & 56.95602 & 18.04011 silic. Is. & $0.02 \mathrm{~L}$ & $3 \mathrm{~L}$ & $0.1 \mathrm{~L}$ \\
\hline $85 T C 097$ & $\mathbf{S}$ & 57.14360 & 19.08360 silic. Is. & 0.03 & $3 \mathrm{~L}$ & $0.1 \mathrm{~L}$ \\
\hline $85 T C 096$ & $\mathbf{F}$ & 57.14360 & 19.08360 silic. Is. & 0.02 & $3 \mathrm{~L}$ & $0.1 \mathrm{~L}$ \\
\hline $85 T C 084$ & $S$ & 56.84576 & 18.95232 silic. Is. & 0.18 & $3 \mathrm{~L}$ & 0.2 \\
\hline $86 T C 439$ & $\mathbf{F}$ & 55.43780 & 17.67175 silic. Is. & 0.04 & $4 \mathrm{~L}$ & $0.1 \mathrm{~L}$ \\
\hline $85 T C 136$ & $\mathbf{S}$ & 56.05713 & 18.23163 silic. Is. & 0.18 & $3 \mathrm{~L}$ & $0.1 \mathrm{~L}$ \\
\hline $86 T C 009$ & $\mathbf{S}$ & 57.97242 & 16.81861 silic. Is. & $0.00 \mathrm{H}$ & 15 & 0.2 \\
\hline $86 T C 001$ & $\mathbf{F}$ & 58.08130 & 16.67647 silic. Is. & 0.04 & 6 & $0.1 \mathrm{~L}$ \\
\hline $85 T C 171$ & $\mathbf{F}$ & 54.78681 & 17.05312 silic. Is. & 0.20 & $3 \mathrm{~L}$ & $0.1 \mathrm{~L}$ \\
\hline $86 T C 005$ & $\mathbf{F}$ & 58.08130 & 16.67647 silic. Is. & $0.00 \mathrm{H}$ & 32 & $0.1 \mathrm{~L}$ \\
\hline $86 T C 004$ & $\mathbf{F}$ & 58.08130 & 16.67647 silic. Is. & $0.00 \mathrm{H}$ & 18 & $0.1 \mathrm{~L}$ \\
\hline $86 T C 003$ & $\mathbf{F}$ & 58.08130 & 16.67647 silic. Is. & $0.00 \mathrm{H}$ & 210 & 0.1 \\
\hline $86 T C 002$ & $\mathbf{F}$ & 58.08130 & 16.67647 silic. Is. & $0.00 \mathrm{H}$ & 52 & $0.1 \mathrm{~L}$ \\
\hline $86 T C 357$ & $\mathbf{F}$ & 55.64370 & 16.61684 silic. Is. & 0.02 & $4 \mathrm{~L}$ & $0.1 \mathrm{~L}$ \\
\hline $85 T C 178$ & $\mathbf{S}$ & 55.49084 & 17.71980 silic. Is. & $0.00 \mathrm{H}$ & 18 & 1.4 \\
\hline $85 T C 160$ & $\mathbf{S}$ & 55.43778 & 18.85513 silic. Is. & 0.70 & $3 \mathrm{~L}$ & $0.1 \mathrm{~L}$ \\
\hline $85 T C 169$ & $\mathbf{S}$ & 55.43778 & 18.85513 silic. Is. & 0.80 & $3 \mathrm{~L}$ & $0.1 \mathrm{~L}$ \\
\hline $85 T C 168$ & $\mathbf{S}$ & 55.43778 & 18.85513 silic. Is. & 0.02 & $3 \mathrm{~L}$ & $0.1 \mathrm{~L}$ \\
\hline $85 K G 011$ & $\mathbf{F}$ & 56.91622 & 18.11586 silic. Is. & 0.07 & 3 & $0.1 \mathrm{~L}$ \\
\hline $86 T C 383$ & $\mathbf{S}$ & 58.72289 & 13.03837 silic. Is. & 2.40 & $4 L$ & 0.1 \\
\hline $85 T C 049$ & $\mathbf{F}$ & 55.04788 & 17.53353 silic. Is. & 0.07 & 3 & $0.1 \mathrm{~L}$ \\
\hline $86 T C 129$ & $\mathbf{S}$ & 56.97579 & 14.32274 silic. Is. & $0.02 \mathrm{~L}$ & $4 L$ & 0.1 \\
\hline $86 T C 192$ & $\mathbf{S}$ & 56.13137 & 15.06894 silic. Is. & 0.04 & 11 & 0.1 \\
\hline $86 T C 191$ & $S$ & 56.82428 & 14.29108 silic. Is. & $0.02 \mathrm{~L}$ & $4 L$ & $0.1 \mathrm{~L}$ \\
\hline $86 T C 047$ & $\mathbf{S}$ & 57.05764 & 16.07043 silic. Is. & 0.04 & $4 \mathrm{~L}$ & $0.1 \mathrm{~L}$ \\
\hline $86 T C 046$ & $\mathbf{S}$ & 57.05473 & 16.03499 silic. Is. & 0.07 & $4 \mathrm{~L}$ & $0.1 \mathrm{~L}$ \\
\hline $86 T C 058$ & $\mathbf{S}$ & 56.48741 & 15.79713 silic. Is. & $0.00 \mathrm{H}$ & 10 & $0.1 \mathrm{~L}$ \\
\hline $86 T C 057$ & $\mathbf{F}$ & 56.63972 & 15.75233 silic. Is. & 0.07 & $4 \mathrm{~L}$ & $0.1 \mathrm{~L}$ \\
\hline $86 T C 288$ & $\mathbf{S}$ & 55.69482 & 20.06506 silic. Is. & 1.12 & $4 \mathrm{~L}$ & $0.1 \mathrm{~L}$ \\
\hline $85 T C 002$ & $\mathbf{F}$ & 58.34813 & 17.04885 silic. Is. & 0.06 & $3 \mathrm{~L}$ & $0.1 \mathrm{~L}$ \\
\hline $85 T C 005$ & $\mathbf{F}$ & 58.43006 & 16.98712 silic. Is. & $0.00 \mathrm{H}$ & 40 & $0.1 \mathrm{~L}$ \\
\hline
\end{tabular}




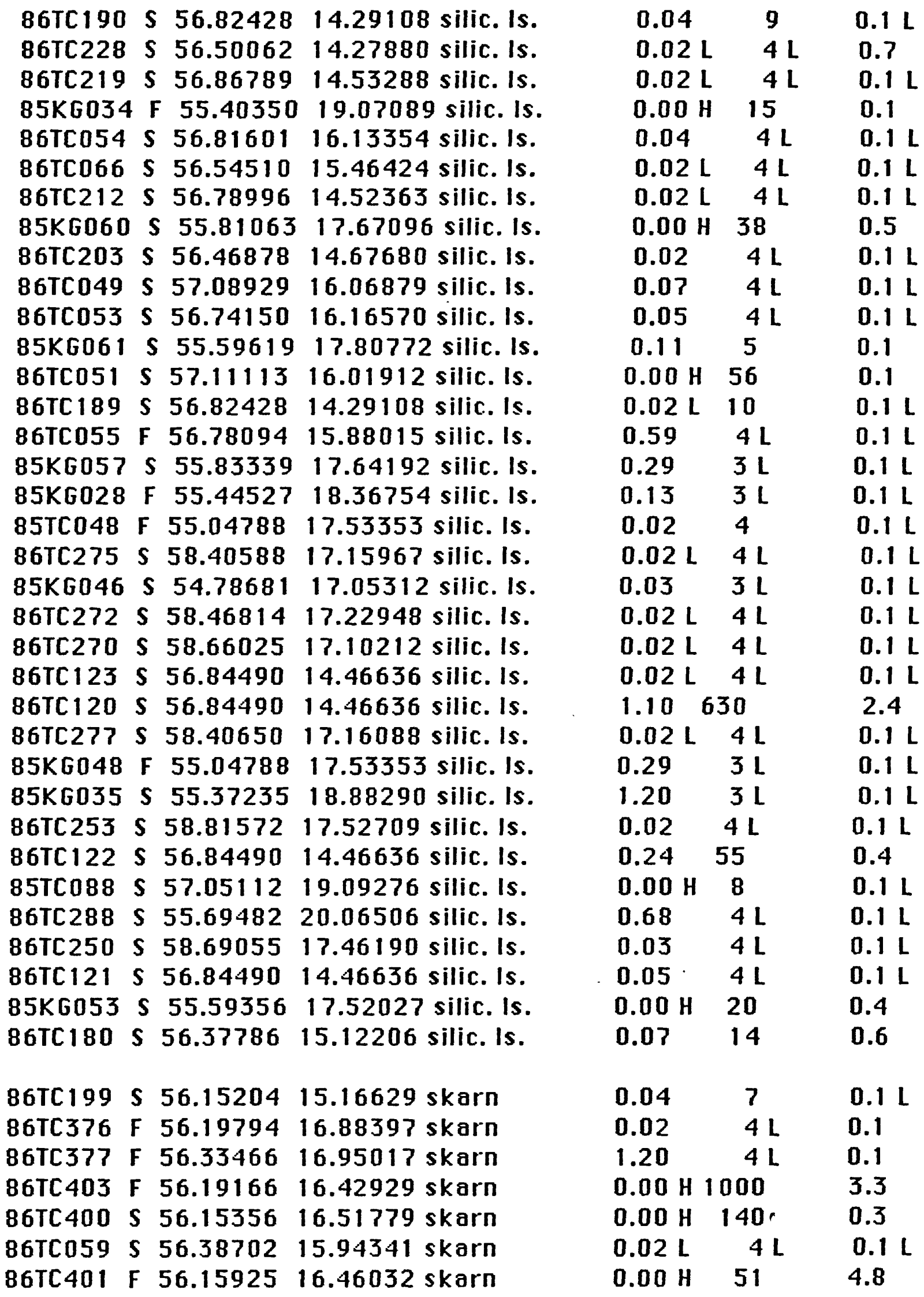




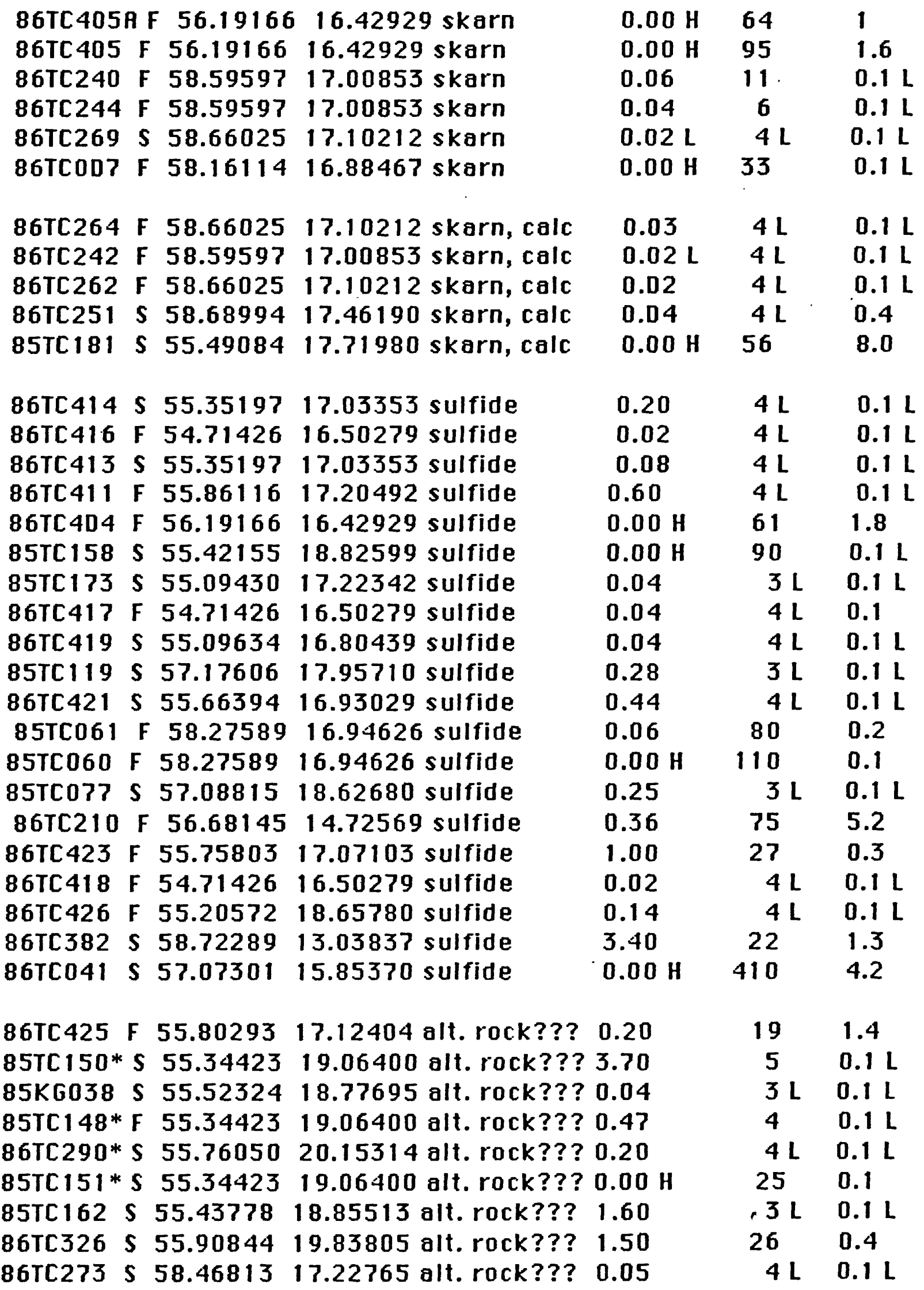

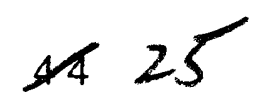




\begin{tabular}{|c|c|c|c|c|c|c|}
\hline $86 \mathrm{TC} 325$ & $\mathbf{S}$ & 55.91292 & 19.94564 alt. rock??? & 0.92 & 15 & 0.7 \\
\hline $86 T C 327$ & $\mathbf{S}$ & 55.90844 & 19.83805 alt. rock??? & 1.40 & 49 & 0.5 \\
\hline $86 T C 330$ & $\mathbf{F}$ & 55.93665 & 19.53306 alt. rock??? & 0.99 & 6.1 & $0.1 \mathrm{~L}$ \\
\hline $86 T C 332$ & $S$ & 56.08716 & 19.62652 alt. rock??? & $0.00 \mathrm{H}$ & 44 & $0.1 \mathrm{~L}$ \\
\hline $35 K^{\prime} 032^{*}$ & $* S$ & 55.39885 & 19.13334 alt. rock??? & 0.53 & 7 & $0.1 \mathrm{~L}$ \\
\hline $35 T C 152 *$ & $S$ & 55.34423 & 19.06400 alt. rock??? & 2.30 & 4 & $0.1 \mathrm{~L}$ \\
\hline $35 K G 008$ & $\mathbf{F}$ & 56.95602 & 18.04011 alt. rock??? & 0.07 & $3 \mathrm{~L}$ & $0.1 \mathrm{~L}$ \\
\hline $5 K G 010$ & $\mathbf{s}$ & 56.95602 & 18.04011 alt. rock??? & 0.52 & $3 \mathrm{~L}$ & $0.1 \mathrm{~L}$ \\
\hline $6 \operatorname{TC} 388 *$ & & 56.33466 & 16.95017 alt. rock??? & 0.40 & 30 & $0.1 \mathrm{~L}$ \\
\hline $35 T C 153^{*}$ & $S$ & 55.34423 & 19.06400 alt. rock??? & 0.90 & $3 L$ & $0.1 \mathrm{~L}$ \\
\hline $85 T C 157$ & $\mathbf{S}$ & 55.42155 & 18.82599 alt. rock??? & $0.00 \mathrm{H}$ & 31 & 0.2 \\
\hline $36 T C 389$ & $\mathbf{S}$ & 56.33466 & 16.95017 alt. rock??? & 0.96 & 7 & $0.1 \mathrm{~L}$ \\
\hline $36 \mathrm{TC} 392$ & $\mathbf{F}$ & 56.76633 & 16.9 & 0.16 & 72 & $0.1 \mathrm{~L}$ \\
\hline $86 T C 187$ & $\mathbf{F}$ & 56.31553 & 15.01584 alt.uolcanic & 0.06 & 91 & 2.0 \\
\hline $86 T C 393$ & $\mathbf{F}$ & 56.81460 & 16.95354 alt.volcanic & 0.20 & $4 L$ & $0.1 \mathrm{~L}$ \\
\hline $85 K G 029$ & $\mathbf{S}$ & 55.38828 & 18.93469 alt.volcanic & 1.20 & $3 \mathrm{~L}$ & $0.1 \mathrm{~L}$ \\
\hline $86 \mathrm{TC} 362$ & $\mathbf{S}$ & 55.72701 & 16.46169 alt.volcanic & 1.76 & 10 & 14.6 \\
\hline $86 T C 234$ & $\mathbf{S}$ & 56.60468 & 19.66360 alt.uolcanic & 0.04 & $4 \mathrm{~L}$ & $0.1 \mathrm{~L}$ \\
\hline $36 T C 391$ & $\mathbf{F}$ & 56.70082 & 17.00968 alt.volcanic & $0.02 \mathrm{~L}$ & 10 & 0.1 \\
\hline $35 K G 036$ & $\mathbf{S}$ & 55.40460 & 18.88004 alt.volcanic & 0.51 & $3 \mathrm{~L}$ & $0.1 \mathrm{~L}$ \\
\hline $86 T C 396$ & $\mathbf{S}$ & 56.64492 & 16.64857 wad & $0.00 \mathrm{H}$ & 18 & 0.7 \\
\hline $86 T C 300$ & $\mathbf{S}$ & 55.64987 & 09 wad & 0.05 & $4 L$ & $0.1 \mathrm{~L}$ \\
\hline $86 T C 305$ & $\mathbf{S}$ & 55.64987 & 20.28309 wad & 0.05 & $4 \mathrm{~L}$ & $0.1 \mathrm{~L}$ \\
\hline $86 T C 306$ & $\mathbf{S}$ & 55.64987 & 20.28309 wad & 0.34 & $4 \mathrm{~L}$ & $0.1 \mathrm{~L}$ \\
\hline $86 T C 045$ & $\mathbf{S}$ & 57.05473 & 16.03499 wad & $0.00 \mathrm{H}$ & 97 & 0.1 \\
\hline $86 \mathrm{TC} 372$ & $\mathbf{F}$ & 56.06989 & 16.82435 mad & $0.02 \mathrm{~L}$ & 36 & 0.4 \\
\hline $86 T C 342$ & $\mathbf{S}$ & 55.41309 & 19.42431 wad & 2.20 & 8 & 1.1 \\
\hline $86 \mathrm{TC} 373$ & $\mathbf{F}$ & 56.06989 & 16.82435 wad & 0.22 & 9 & 2.4 \\
\hline $86 T C 194$ & $\mathbf{F}$ & 56.30563 & $15.23311 \mathrm{wad}$ & 0.12 & $4 L$ & $0.1 \mathrm{~L}$ \\
\hline TTC252 & 3 & 8 & & $\mathbf{u}$ & $4 \mathrm{~L}$ & 0.11 \\
\hline
\end{tabular}




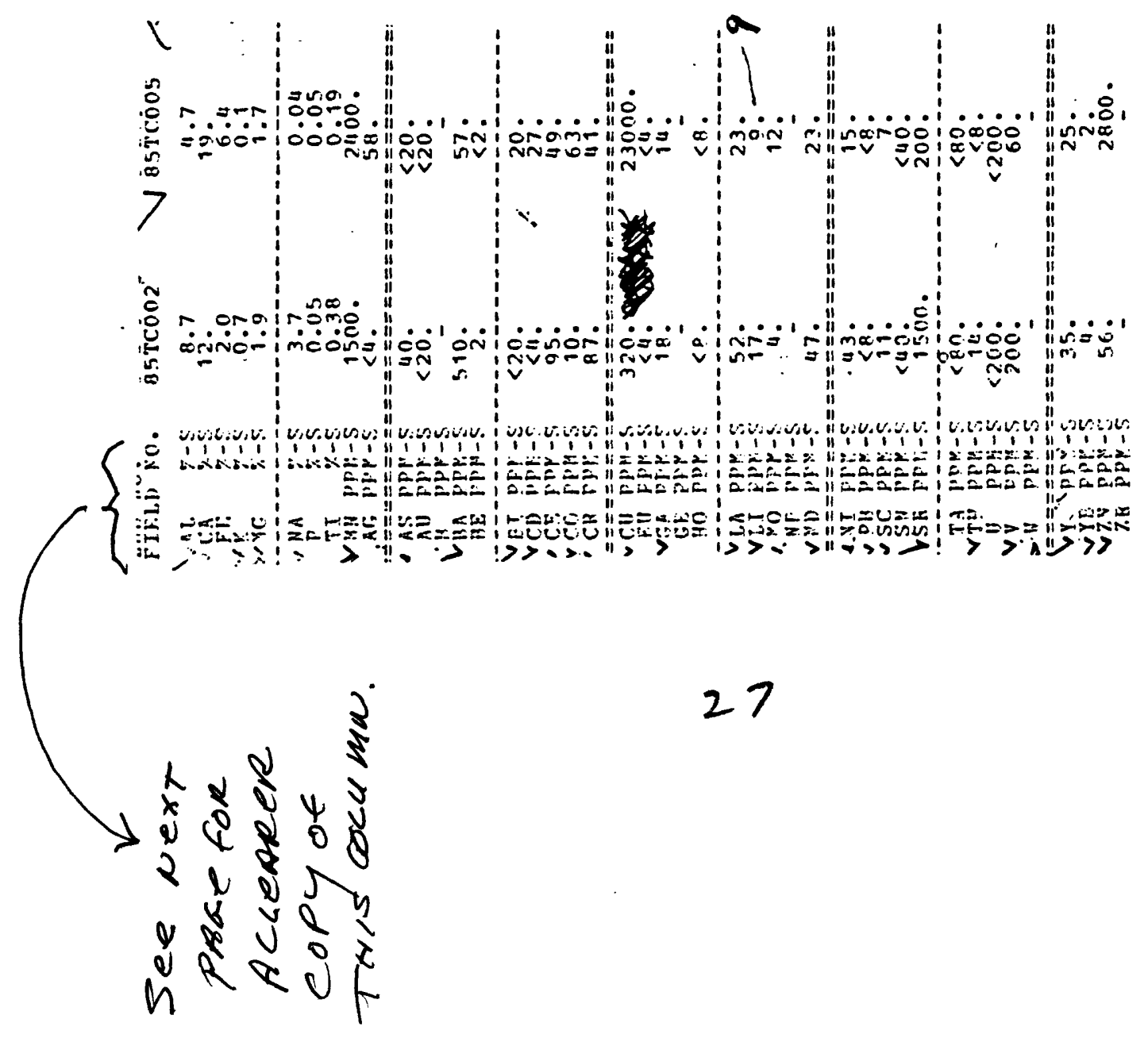



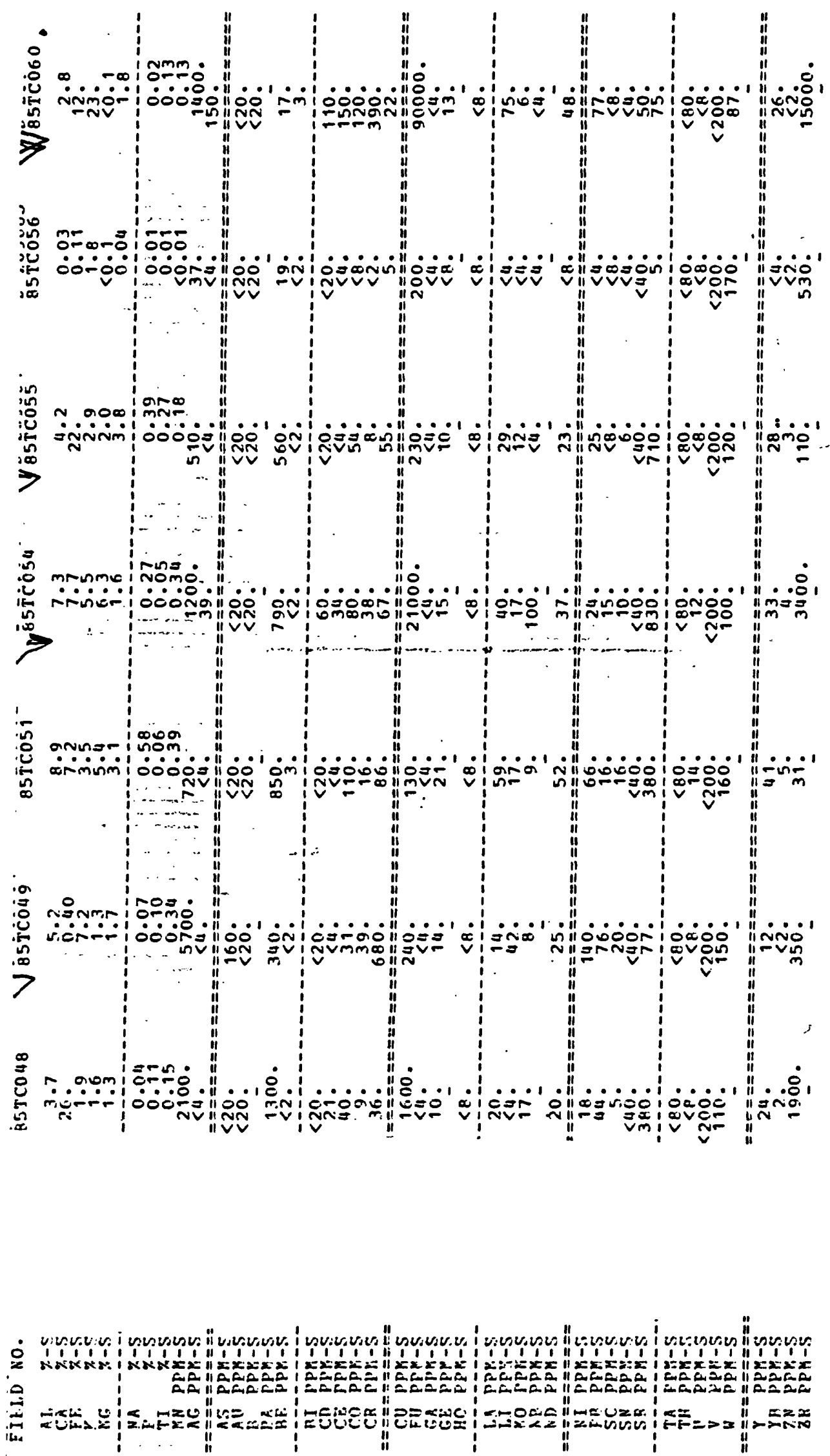


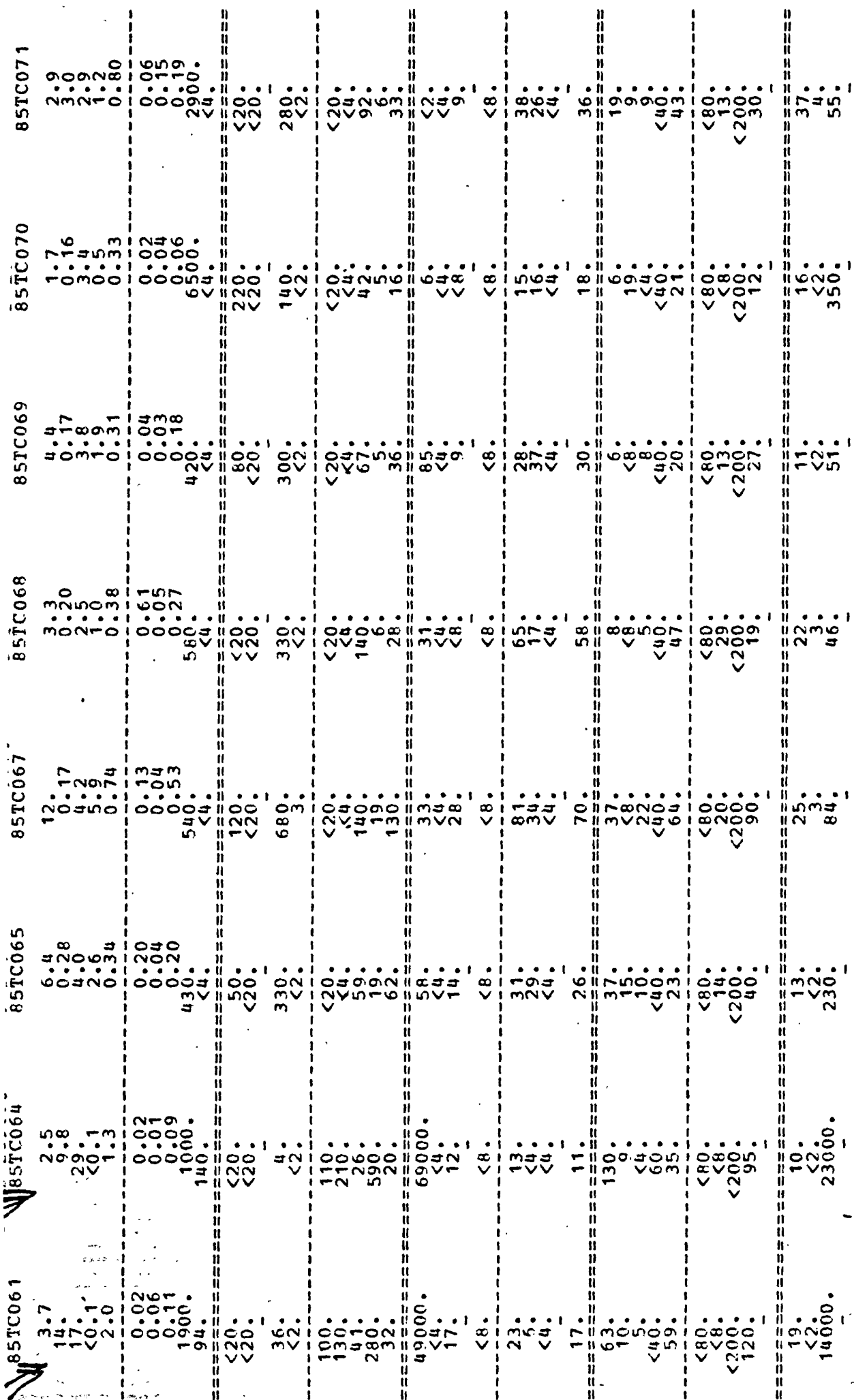

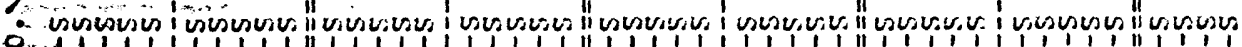

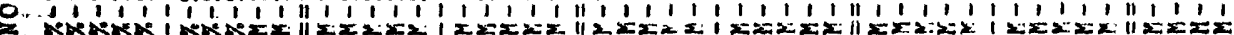

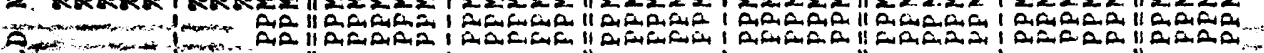

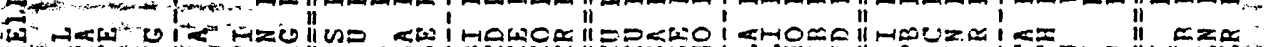

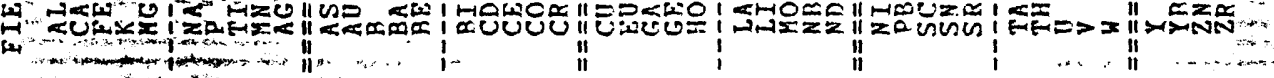

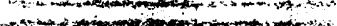




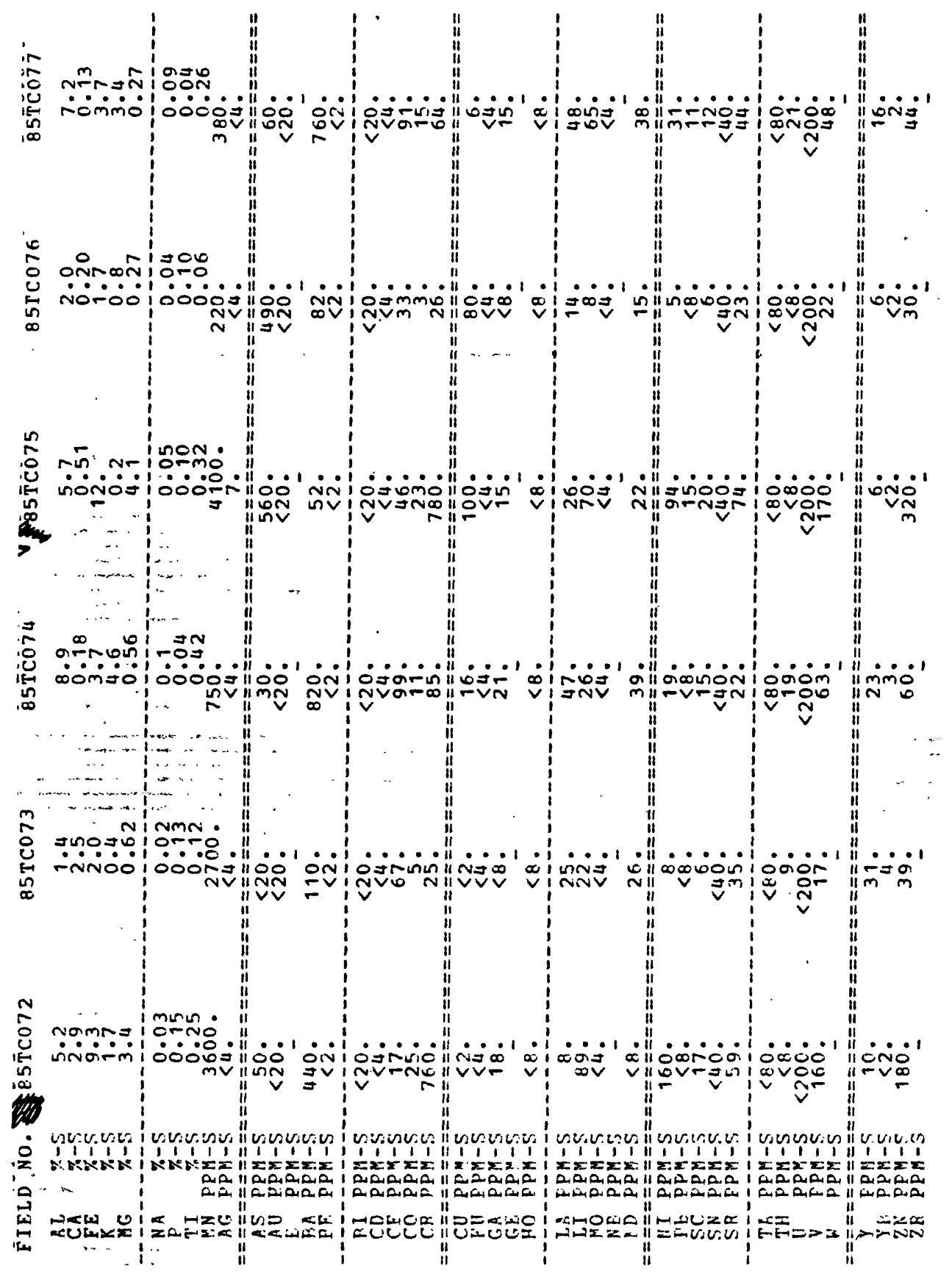




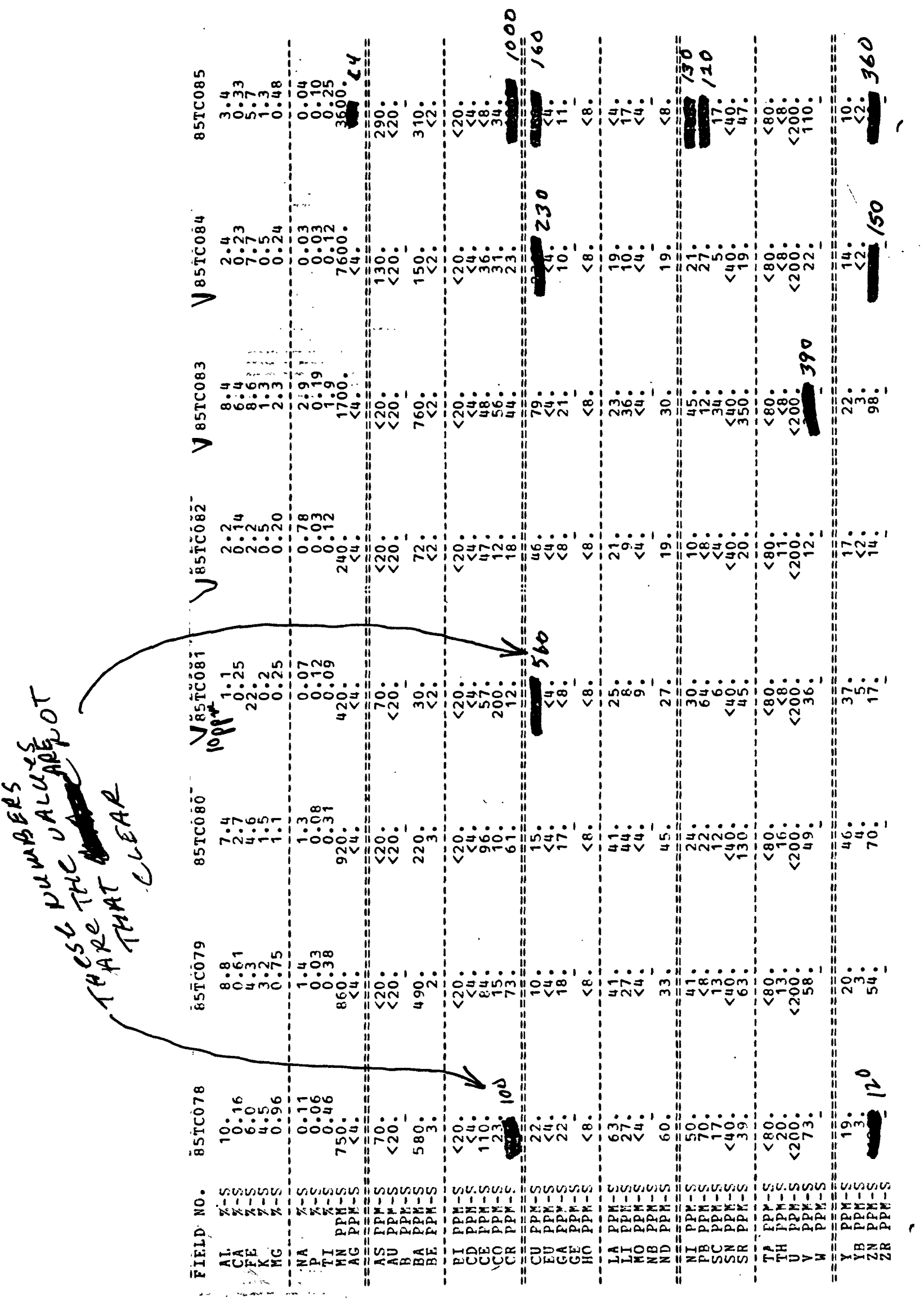




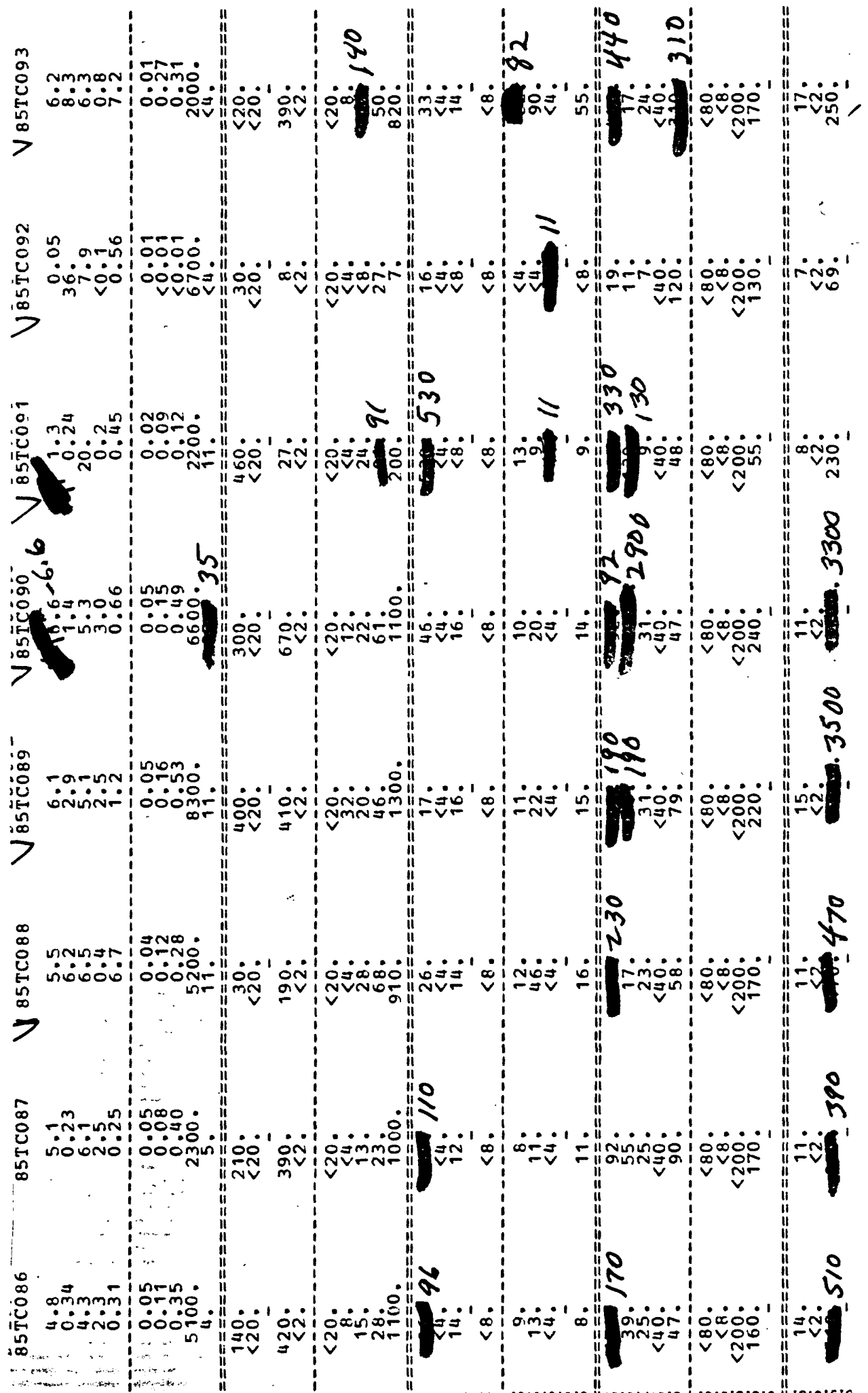

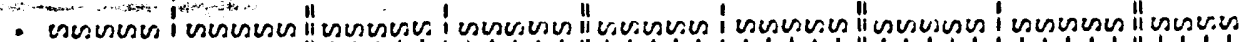

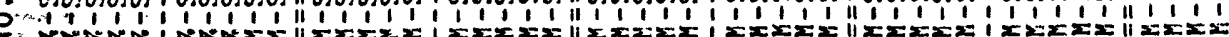
$\begin{array}{lll} & \\ 0 & \end{array}$

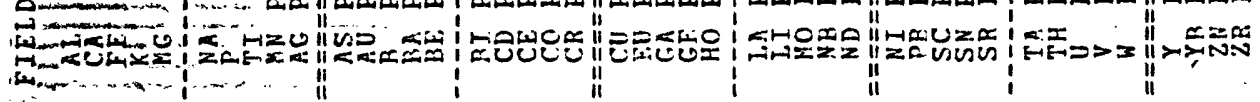




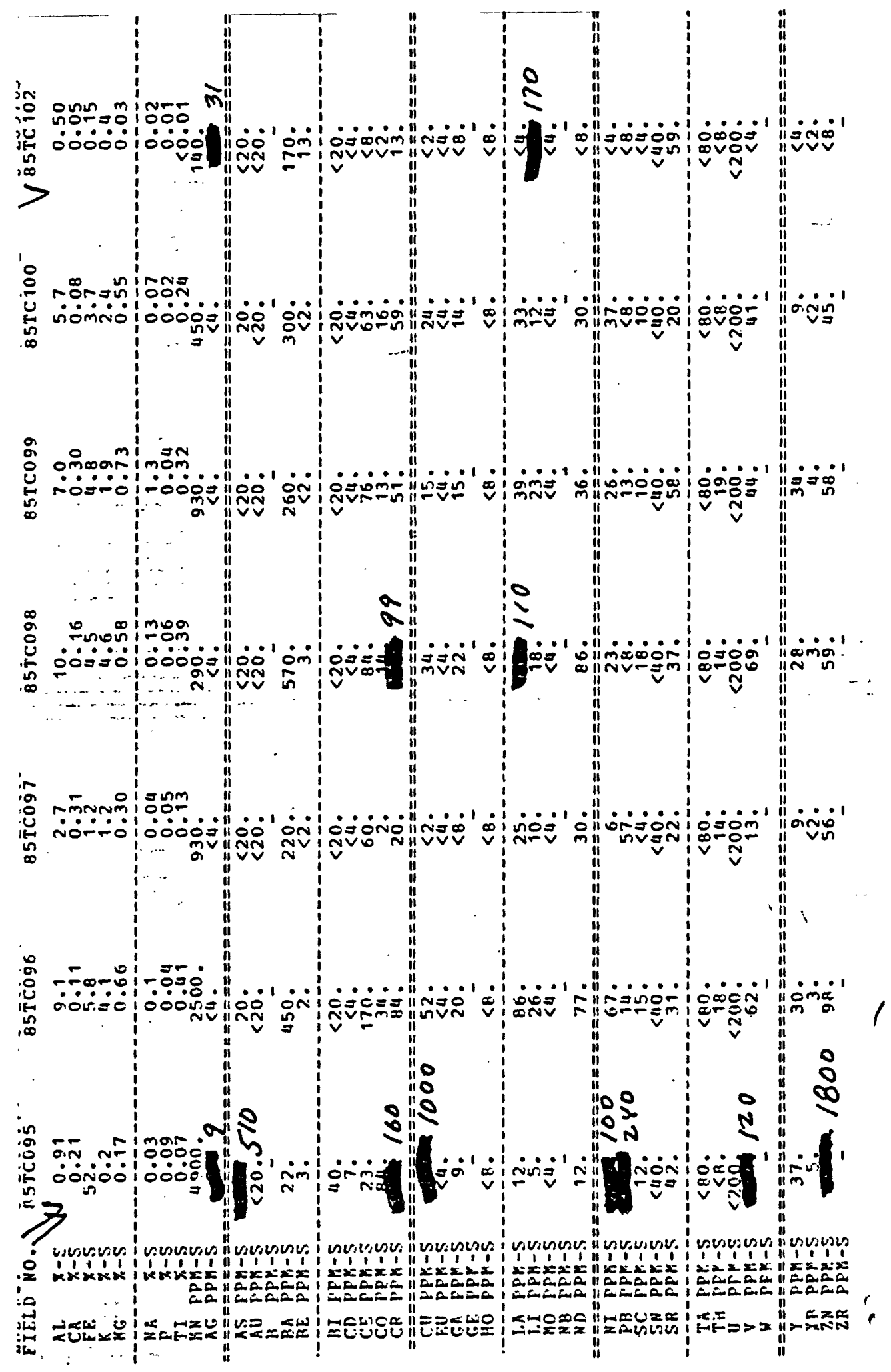



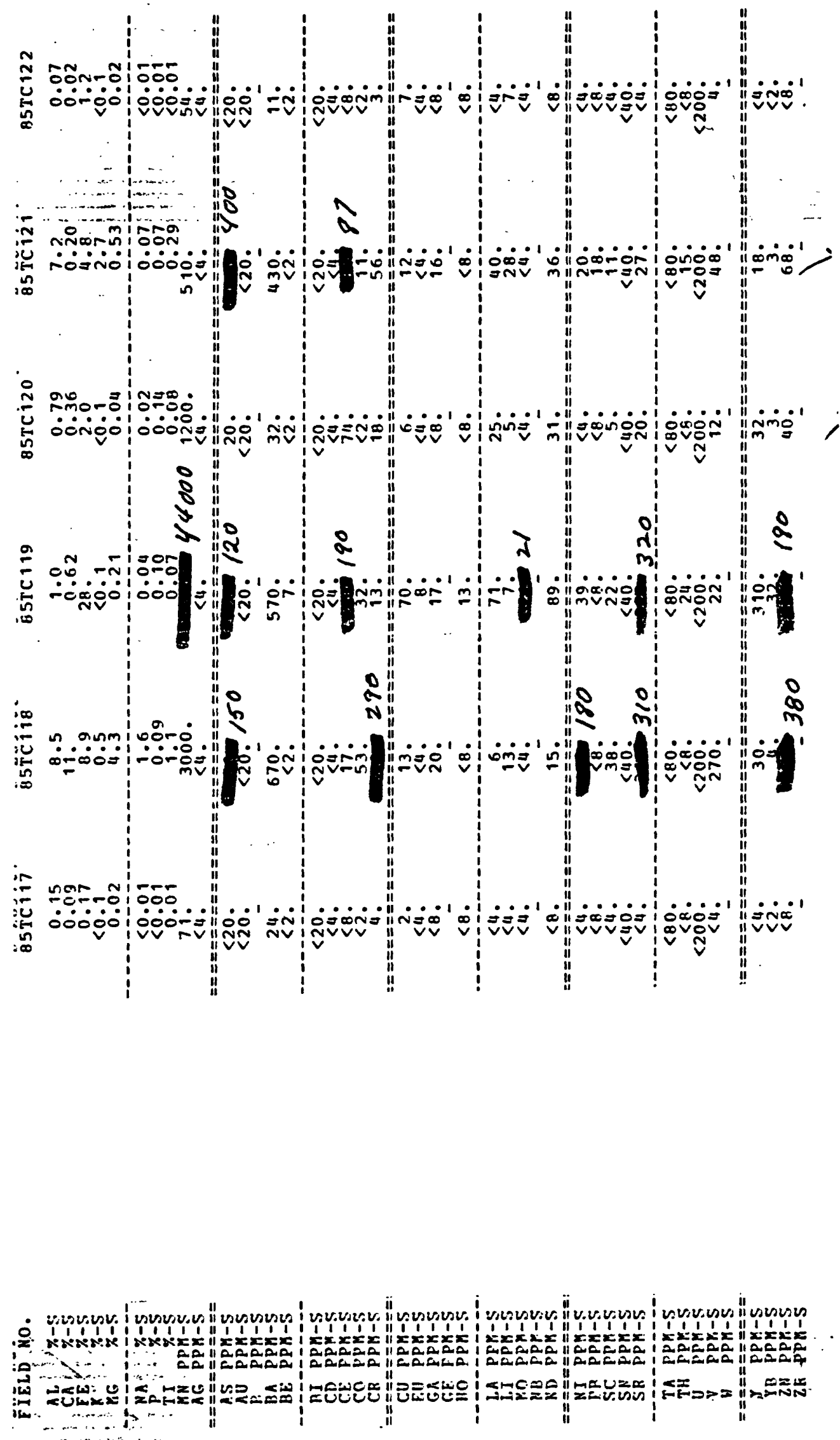


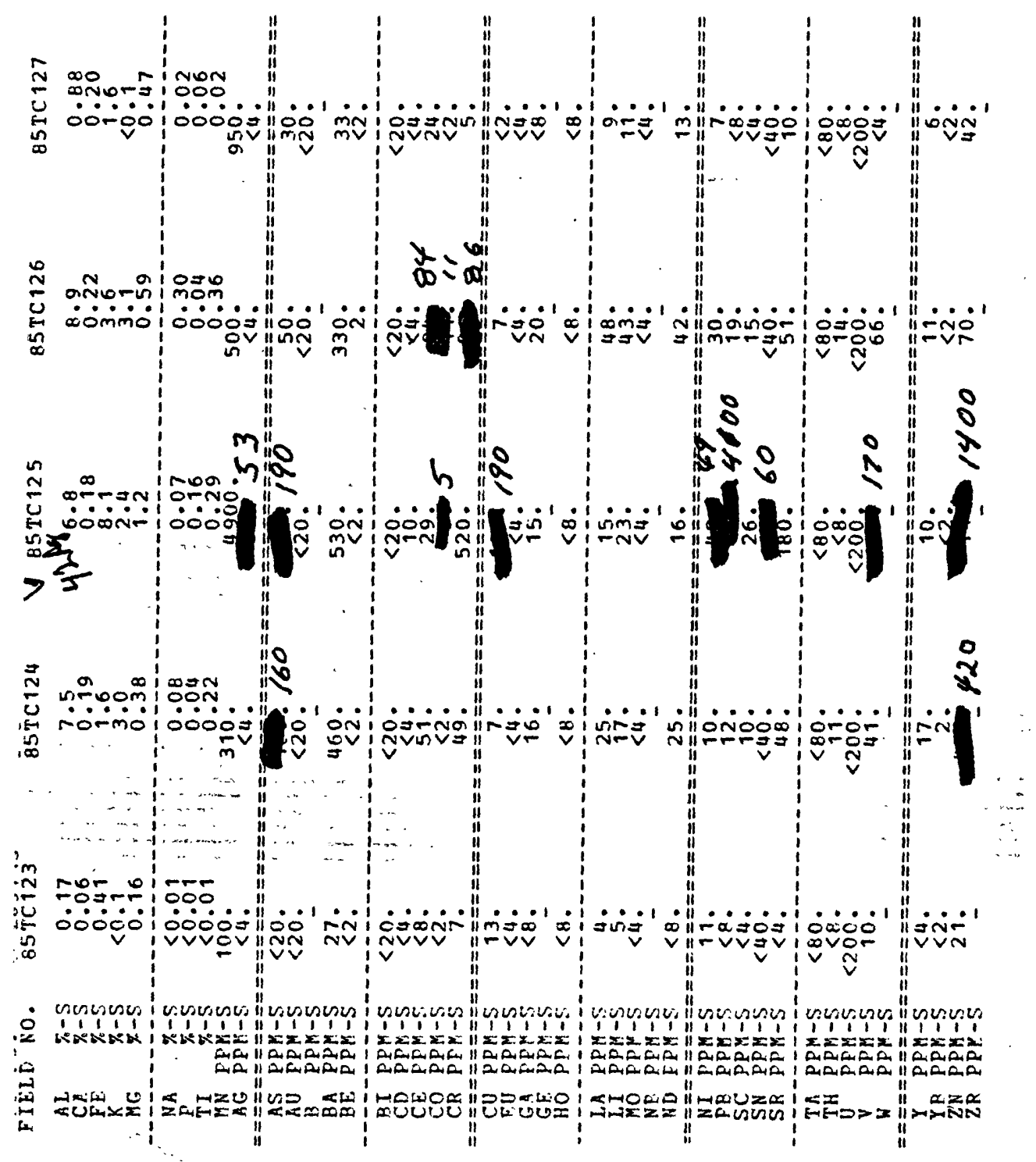




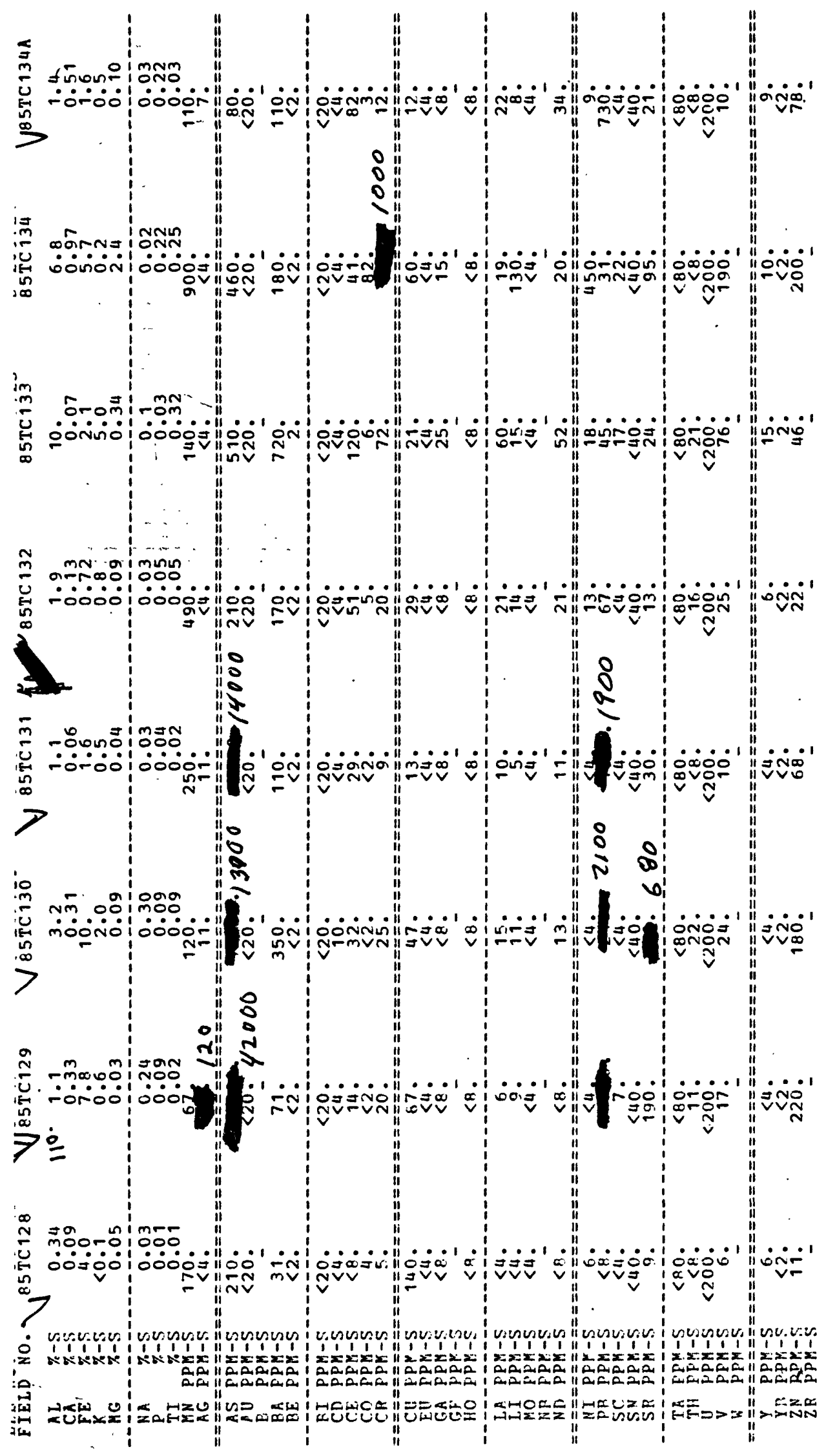




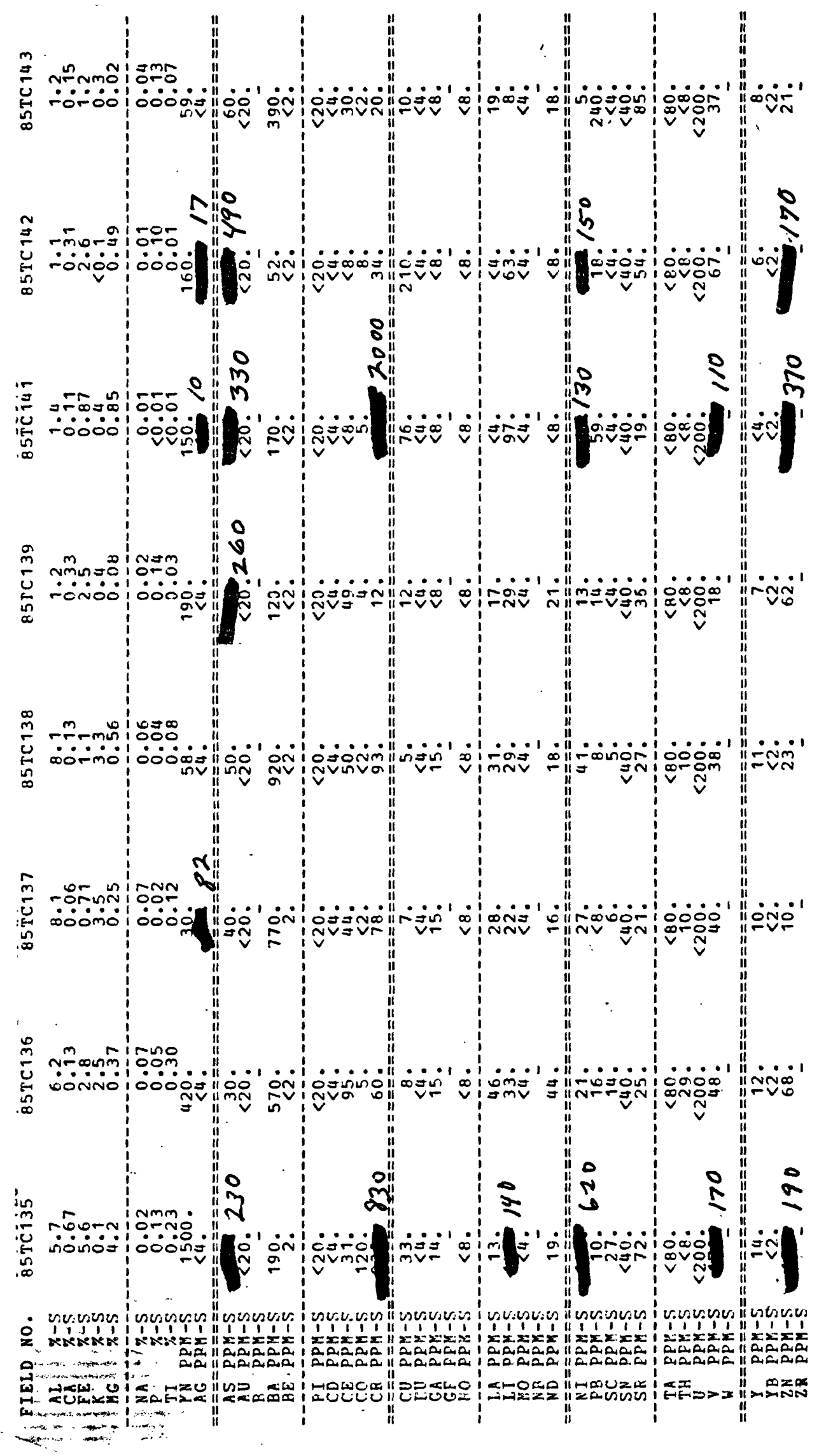




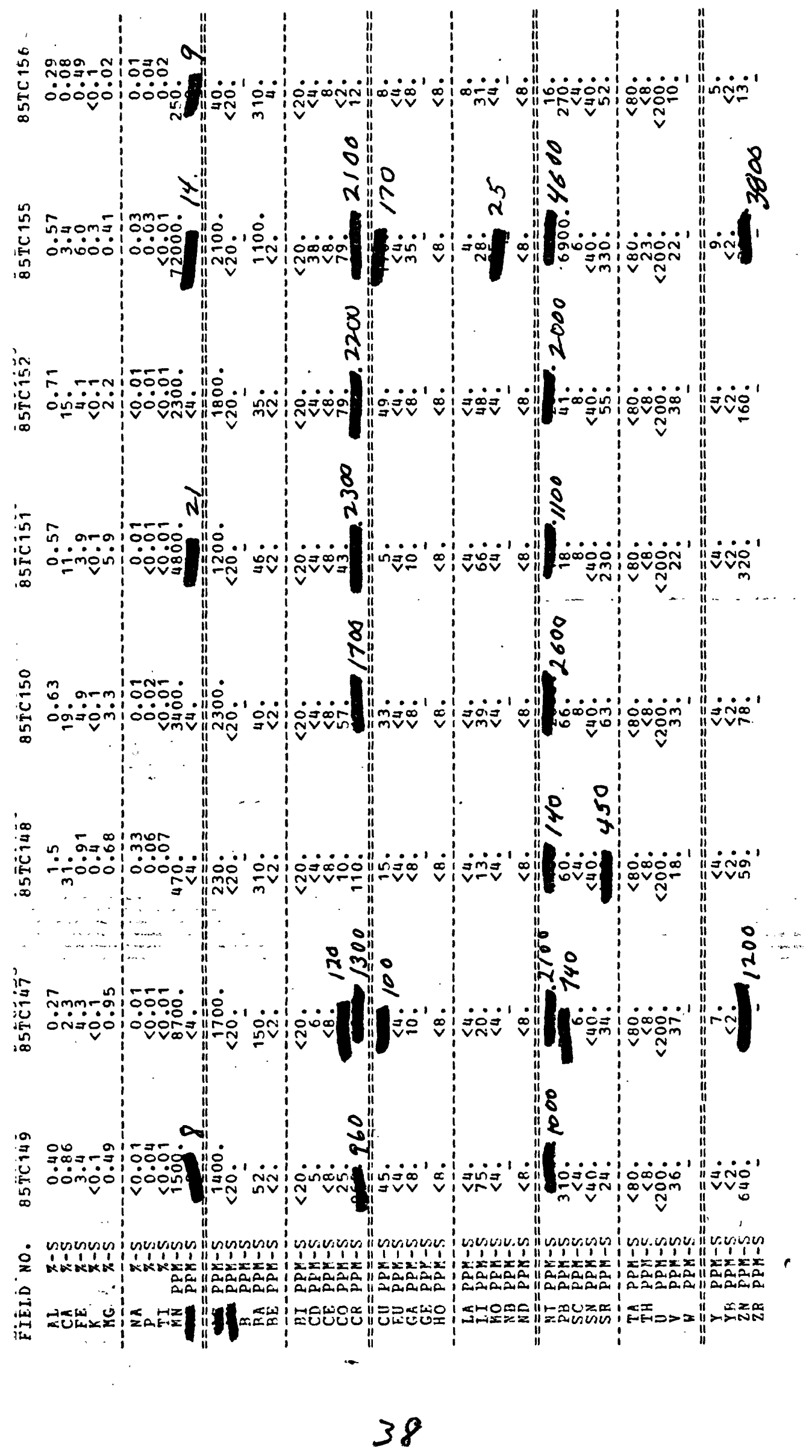




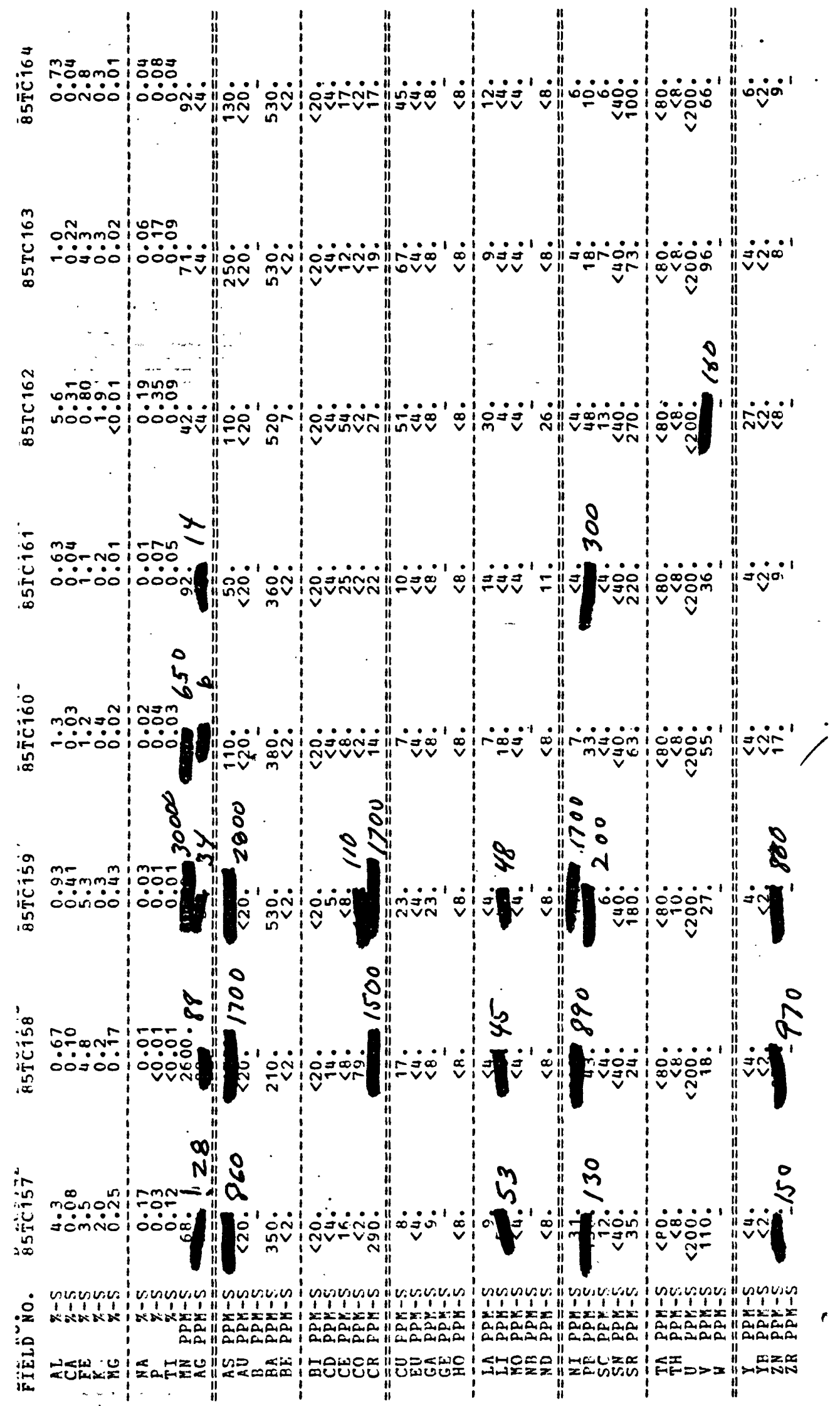




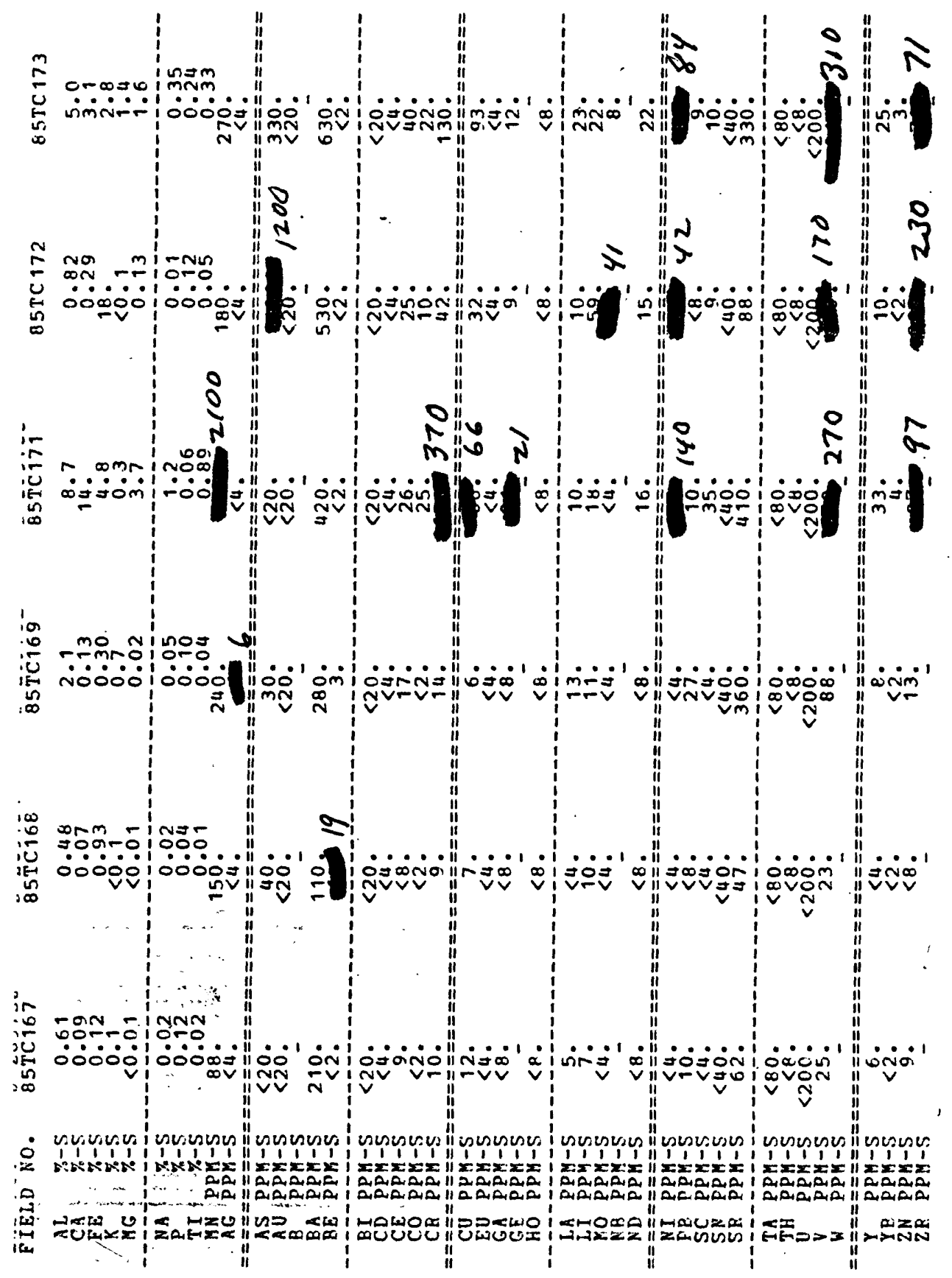




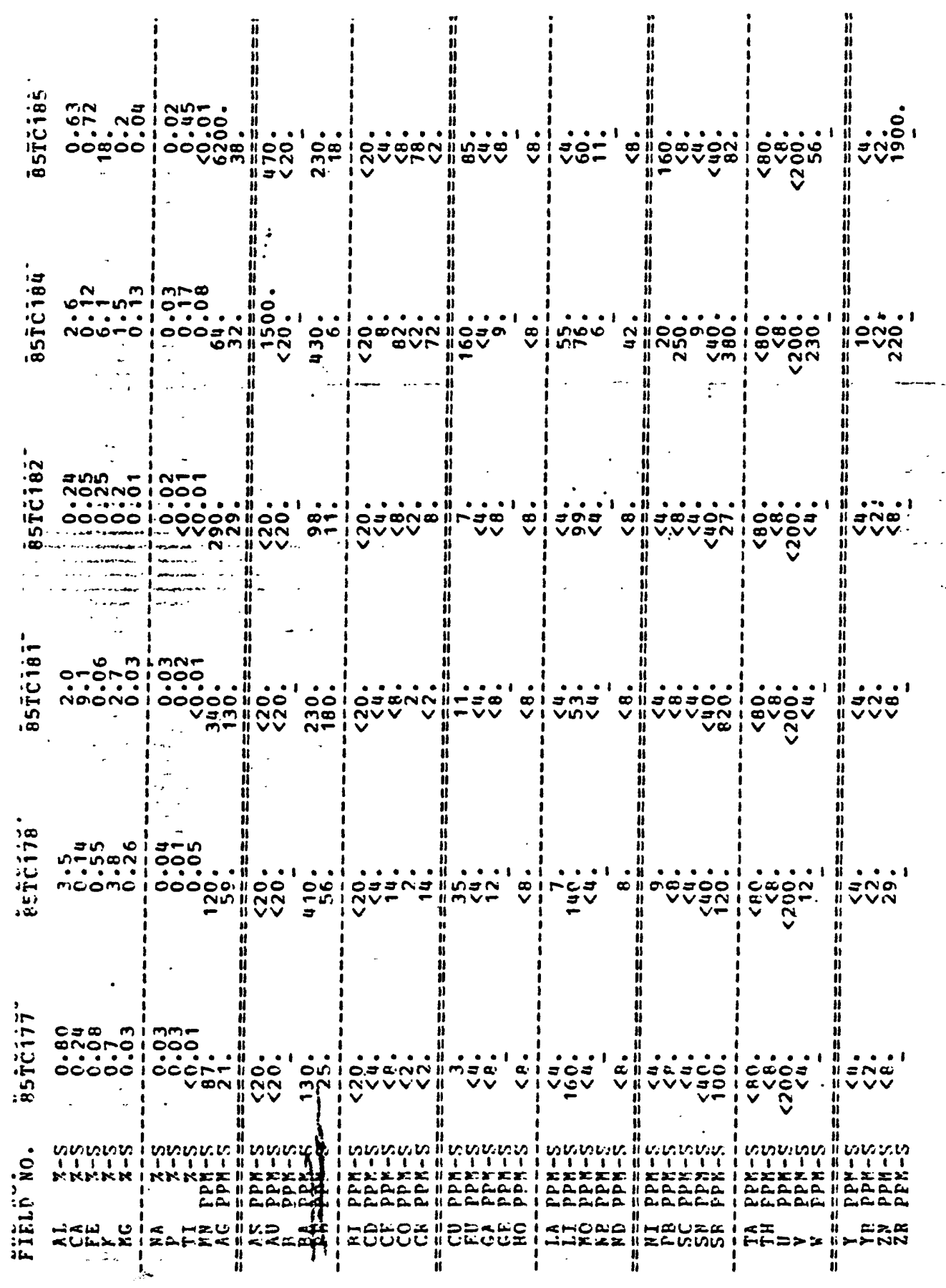




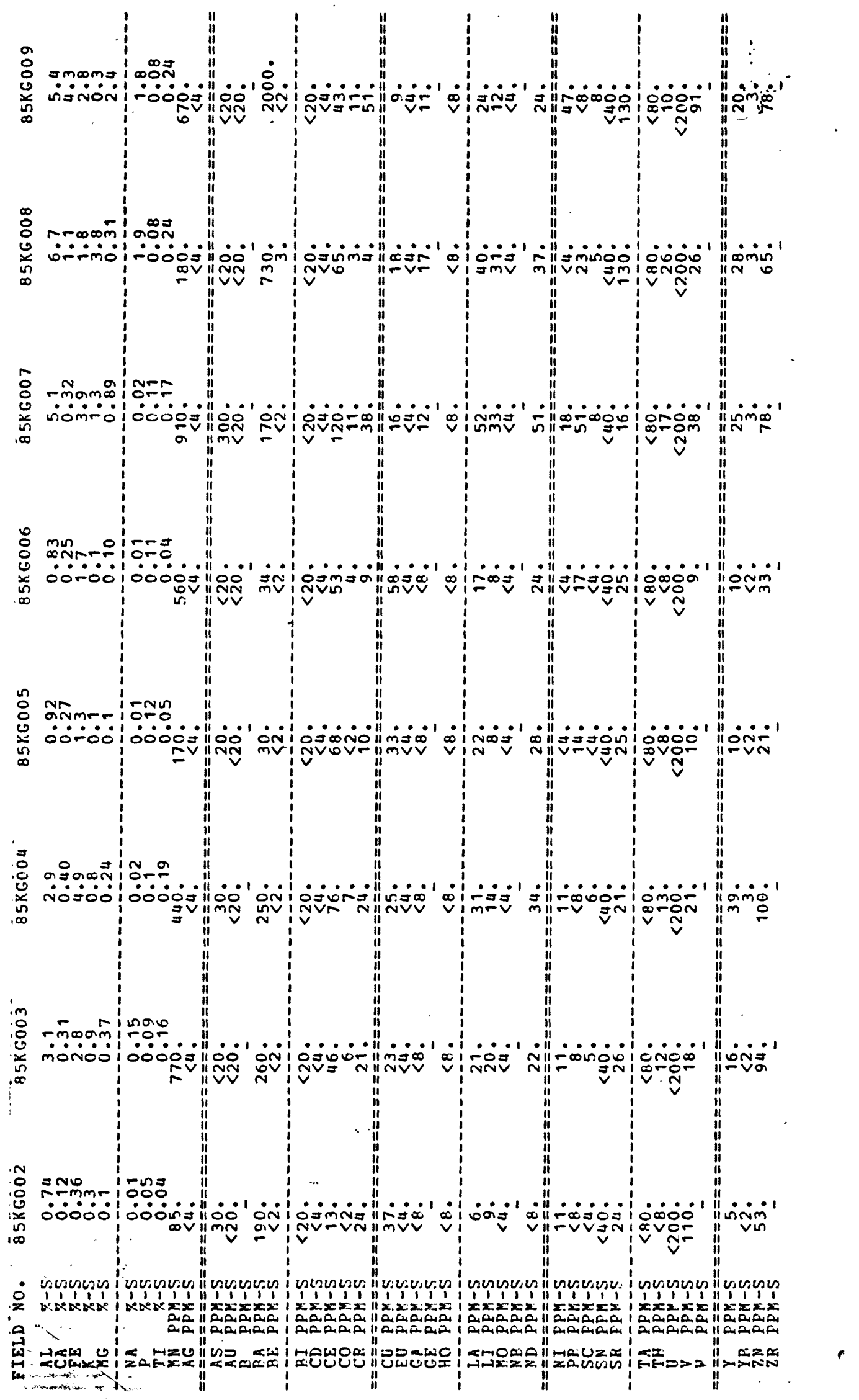




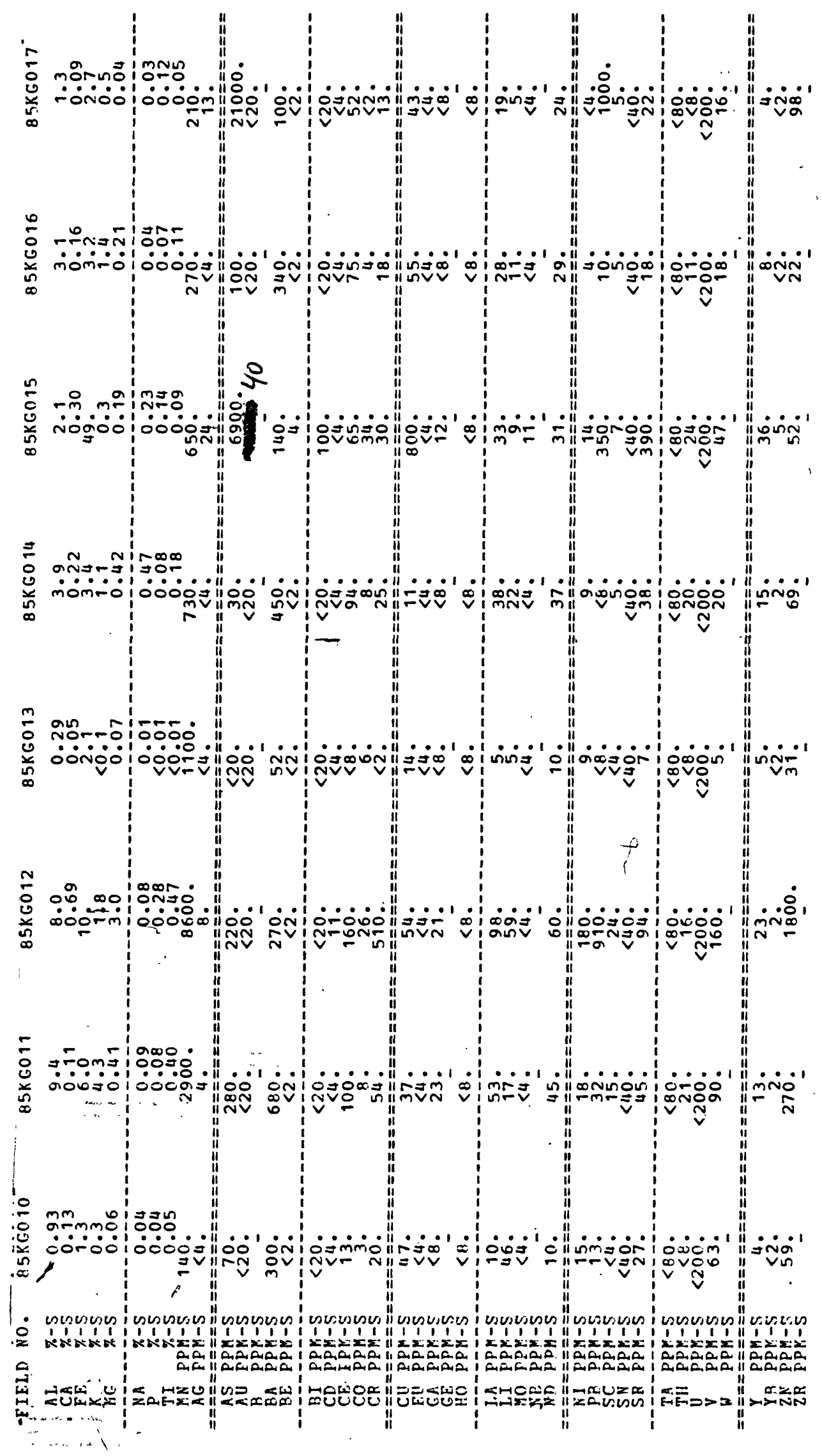




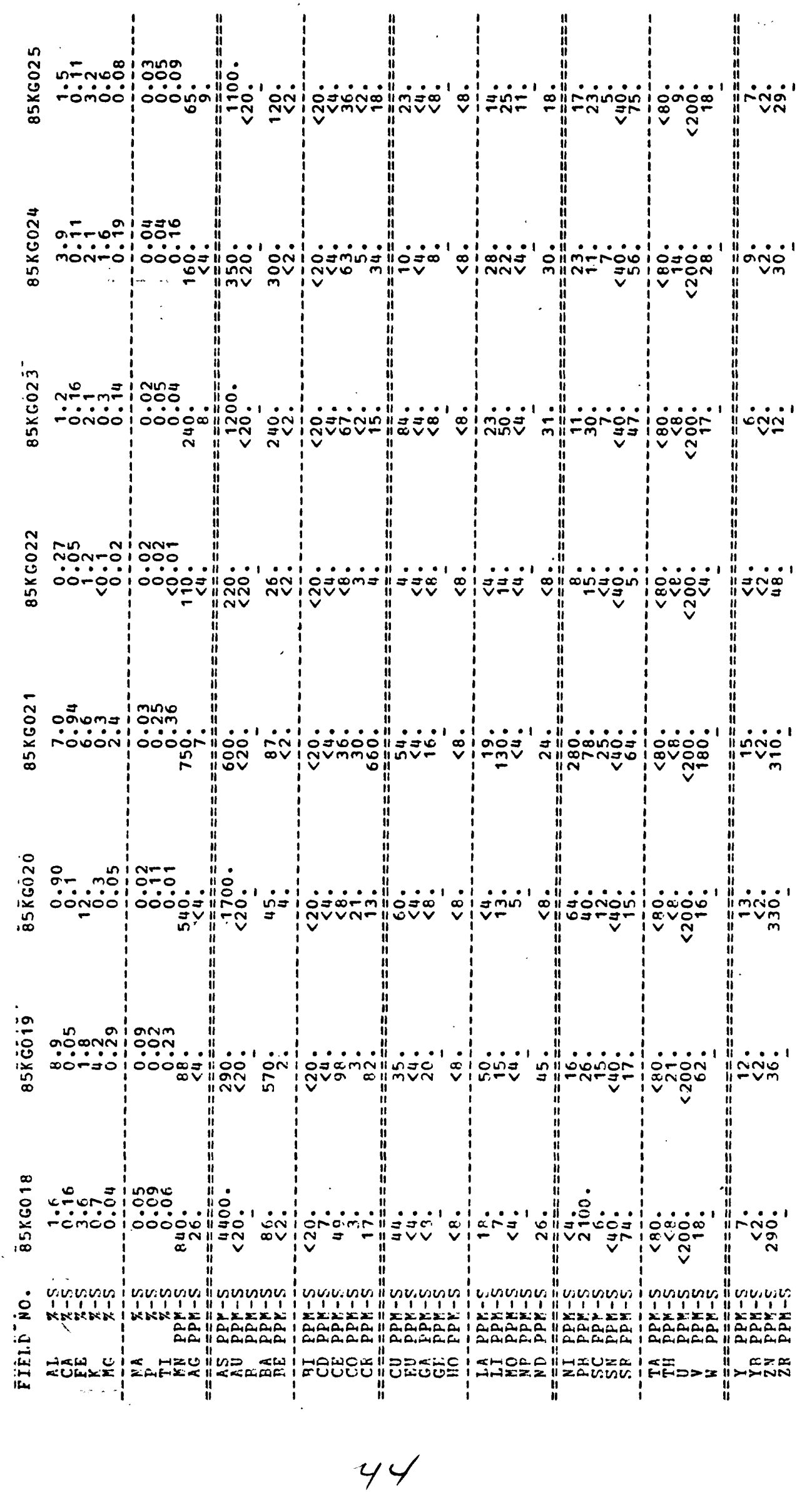




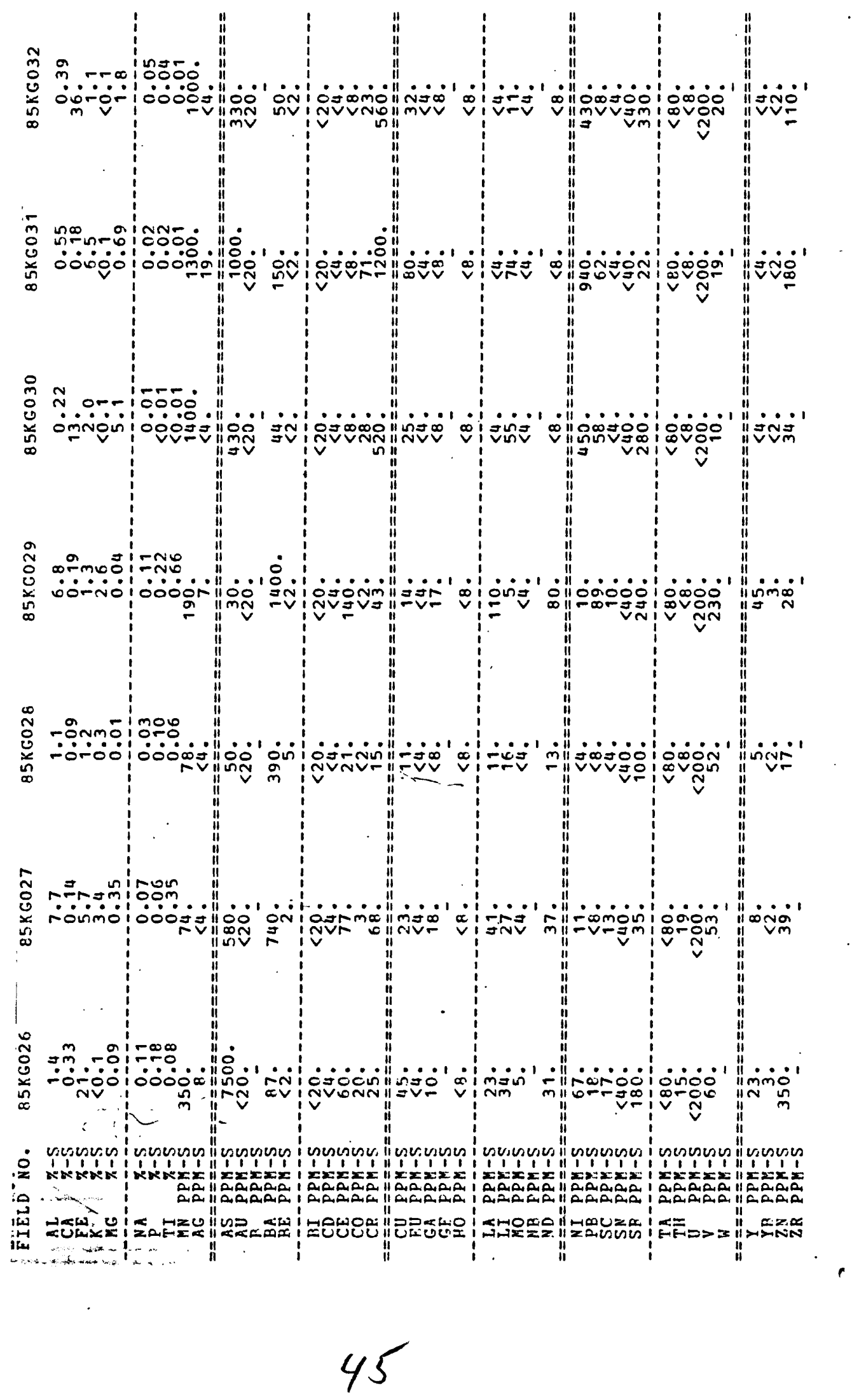




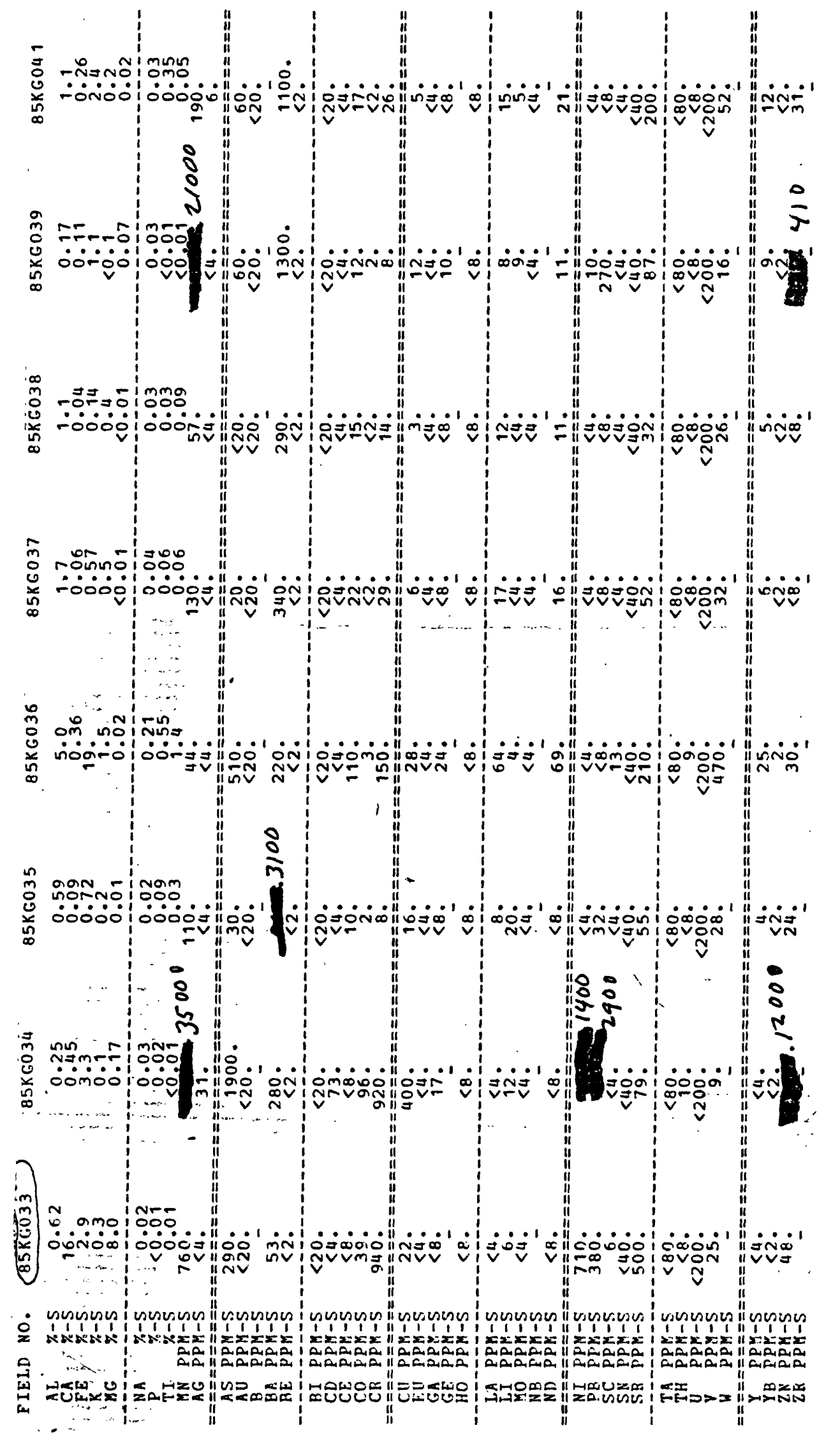




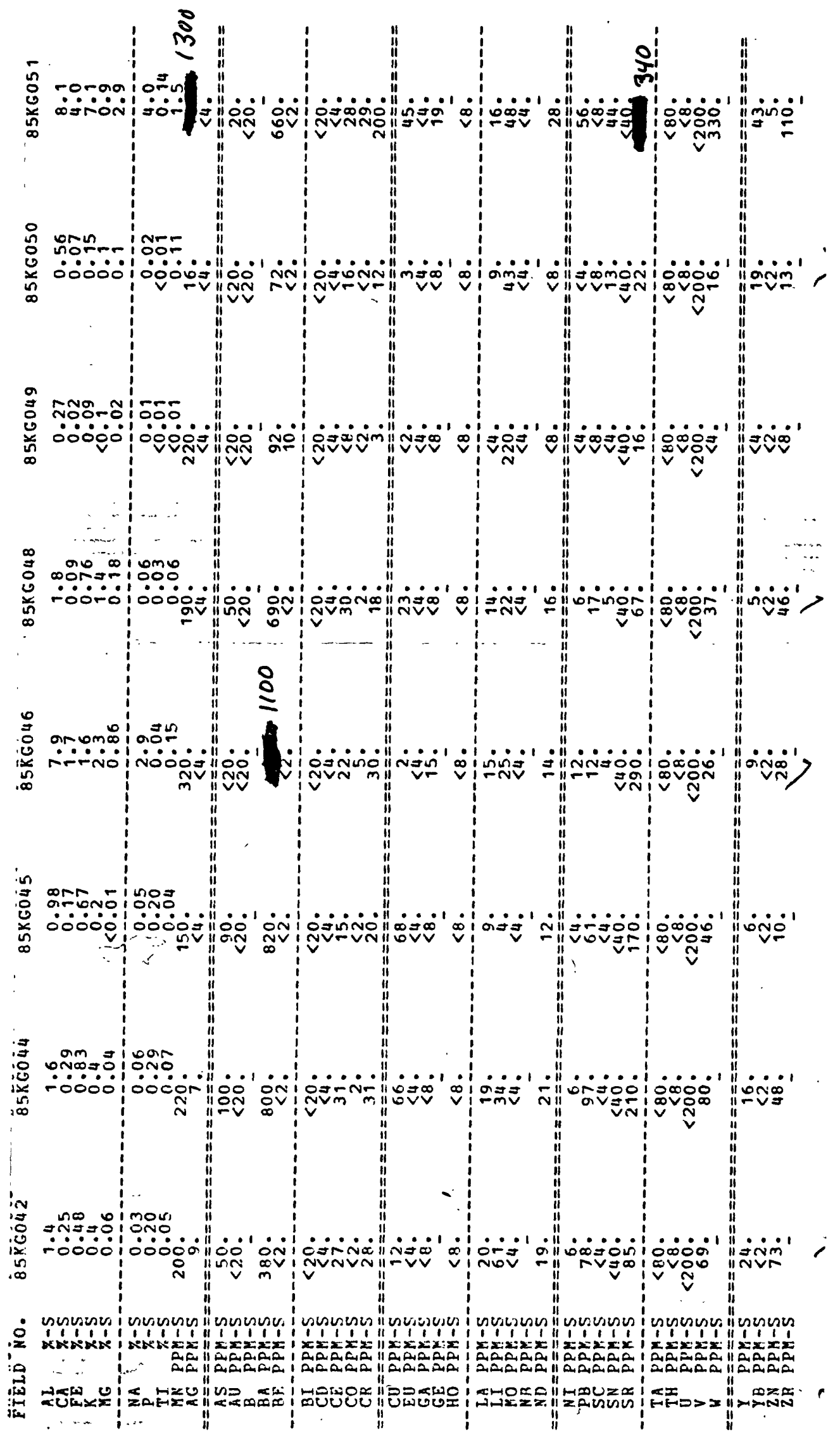




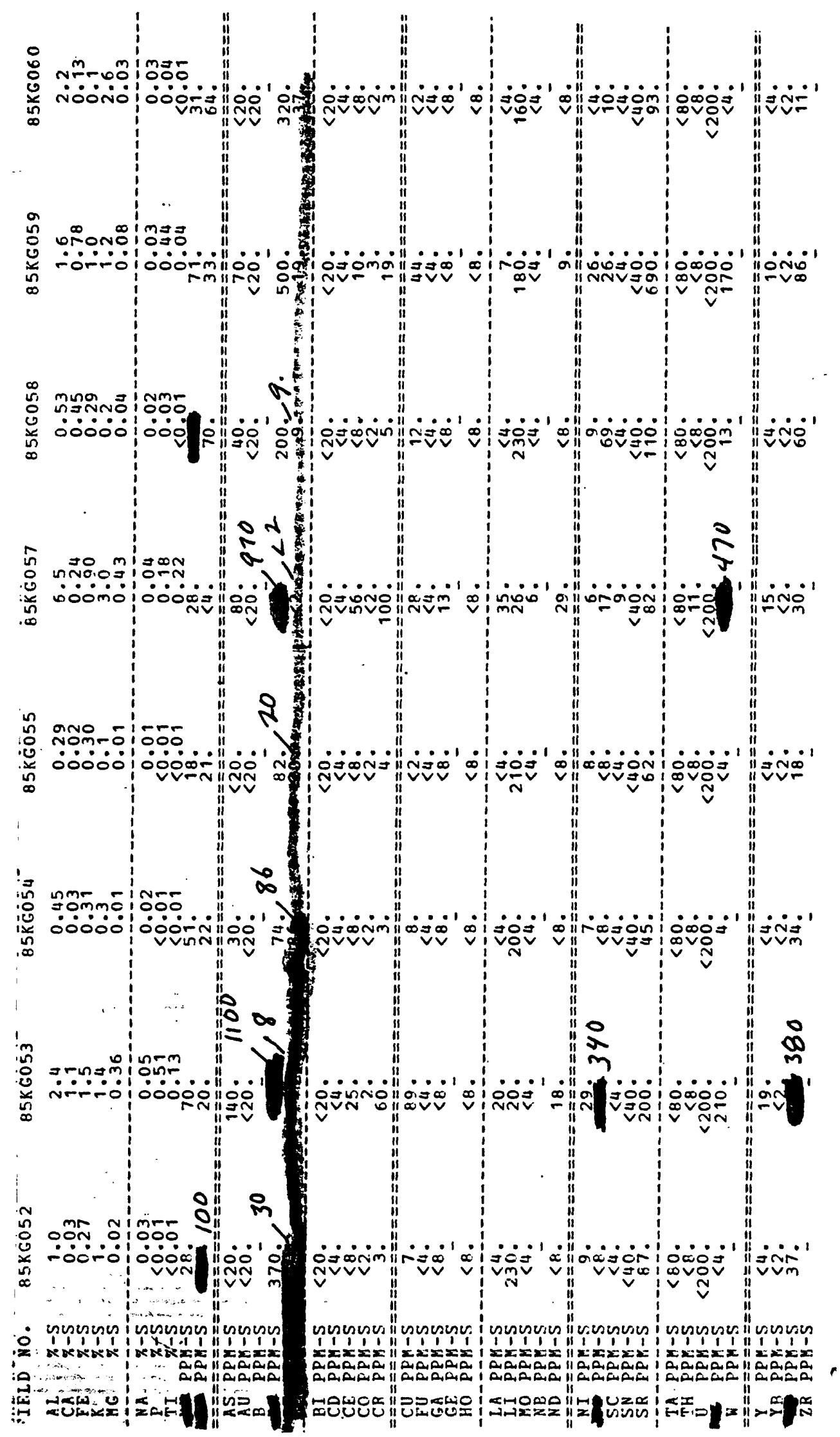




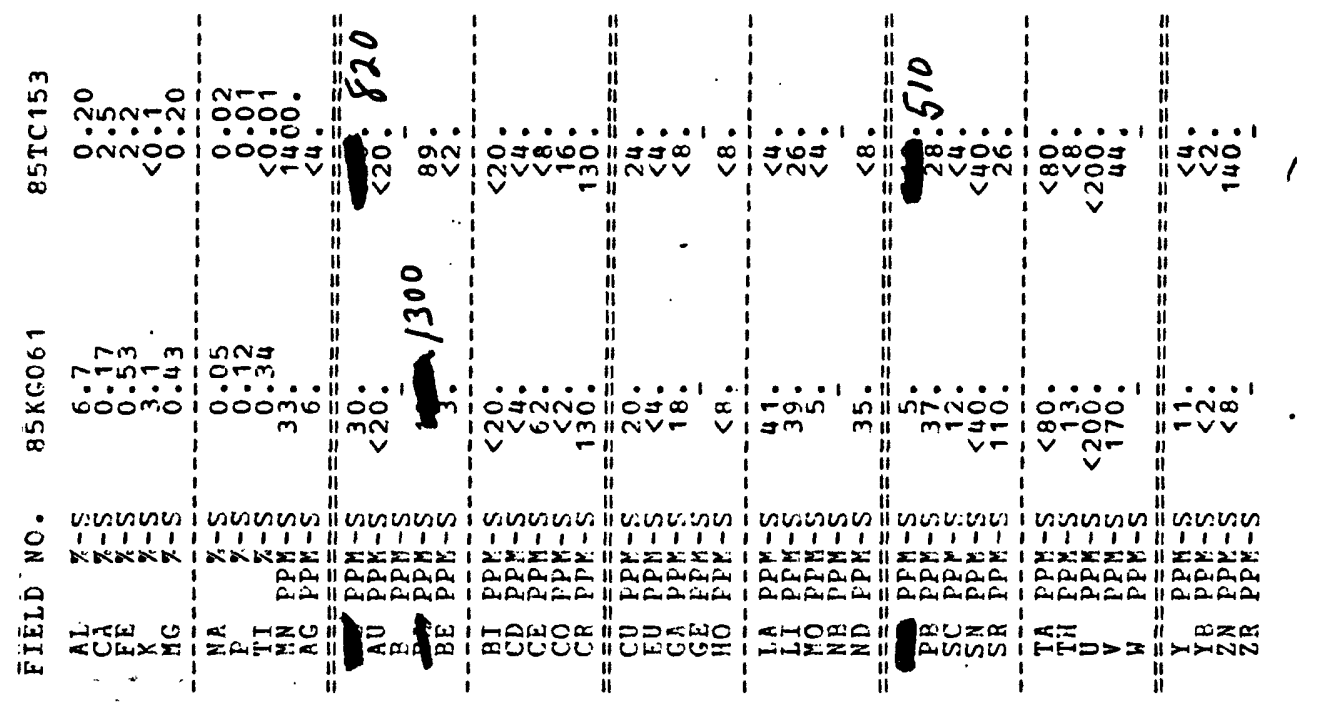




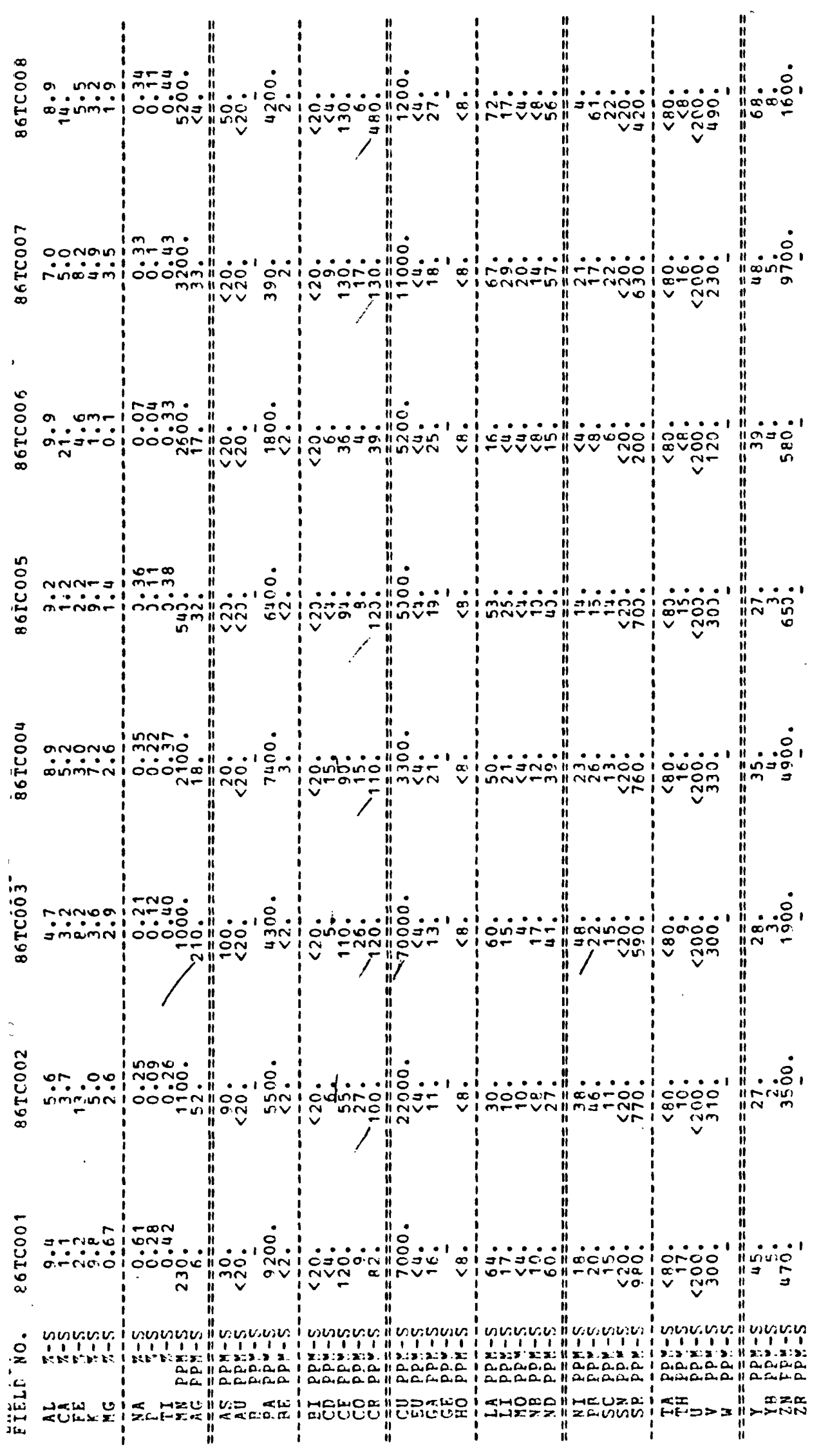




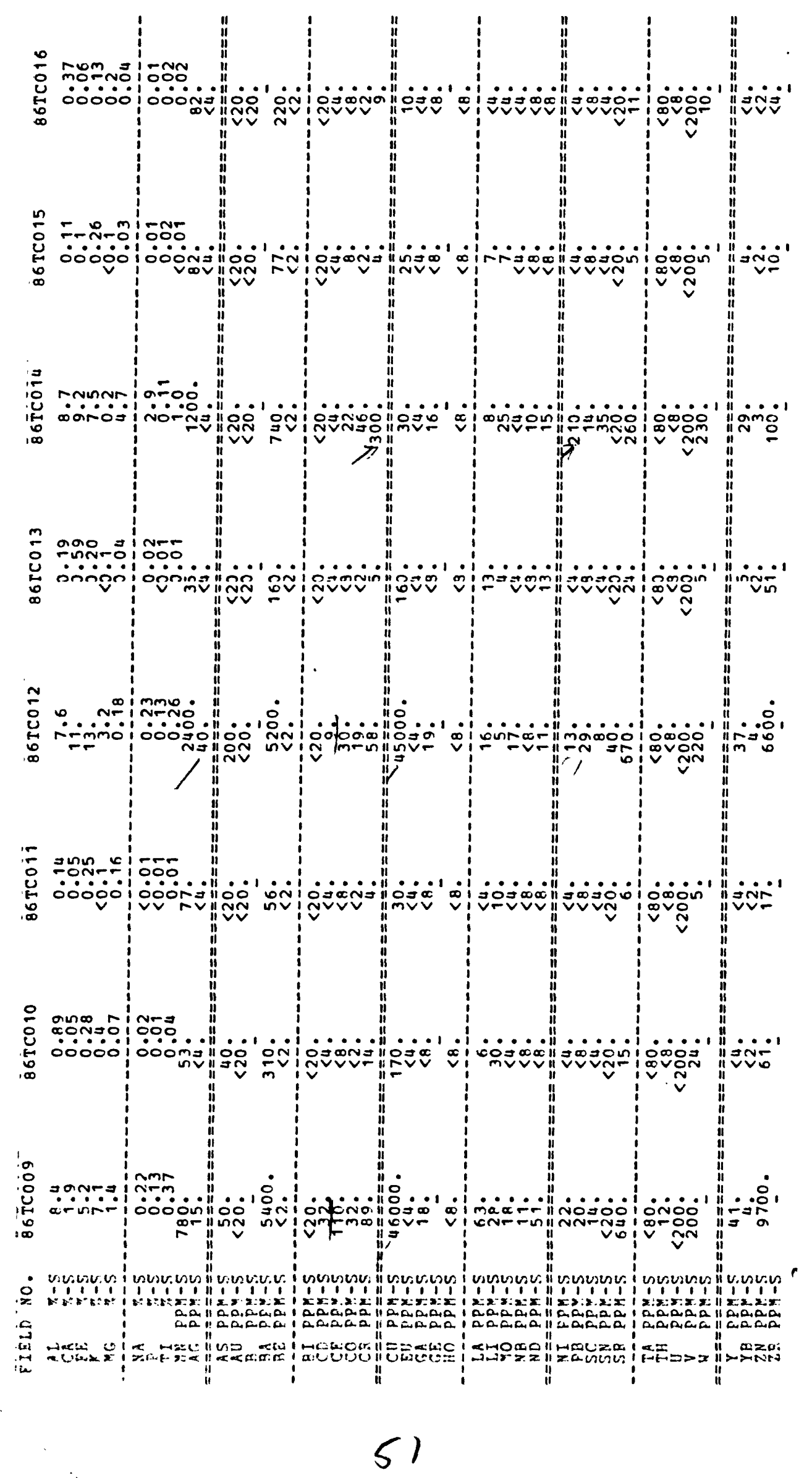




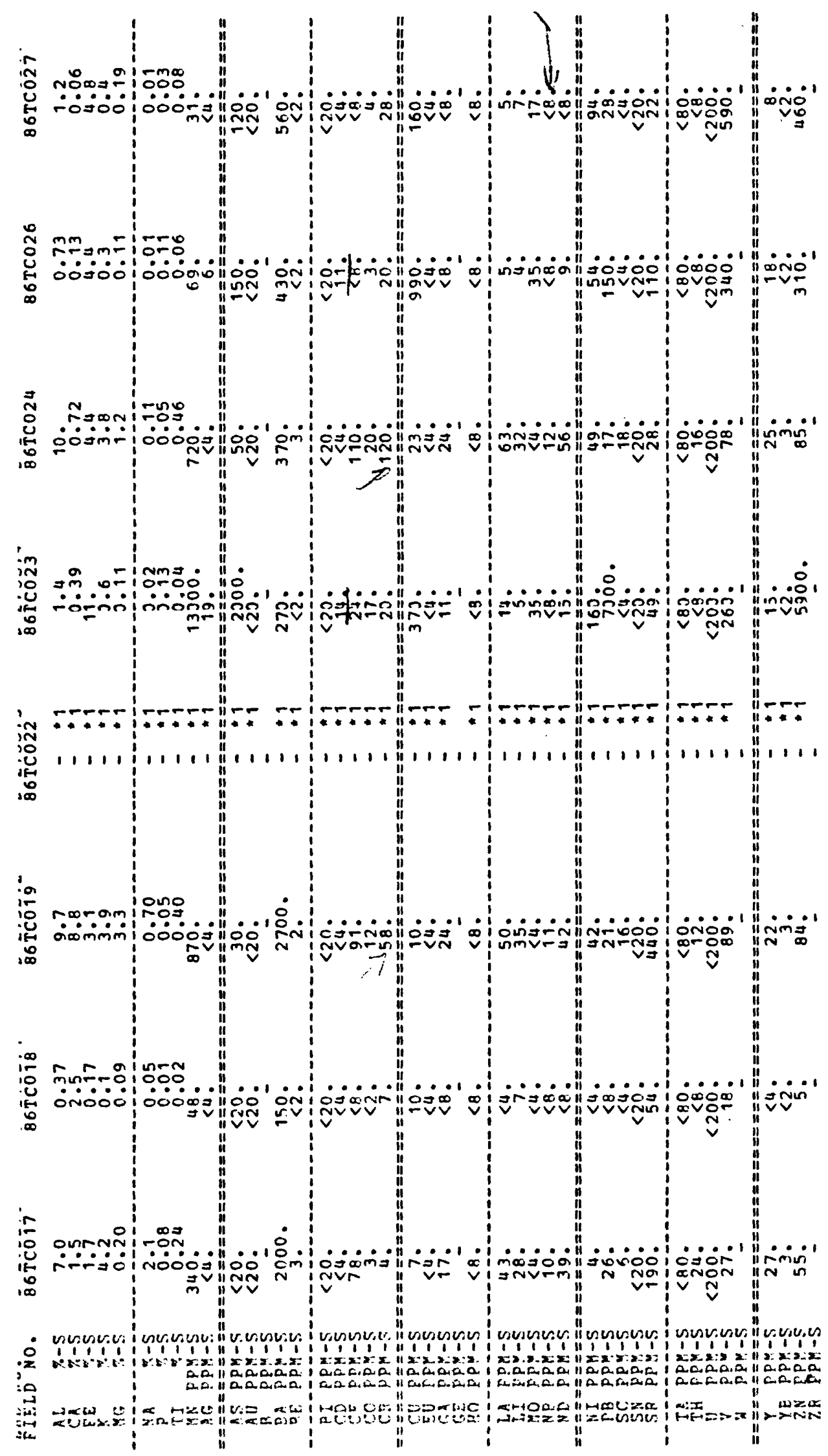




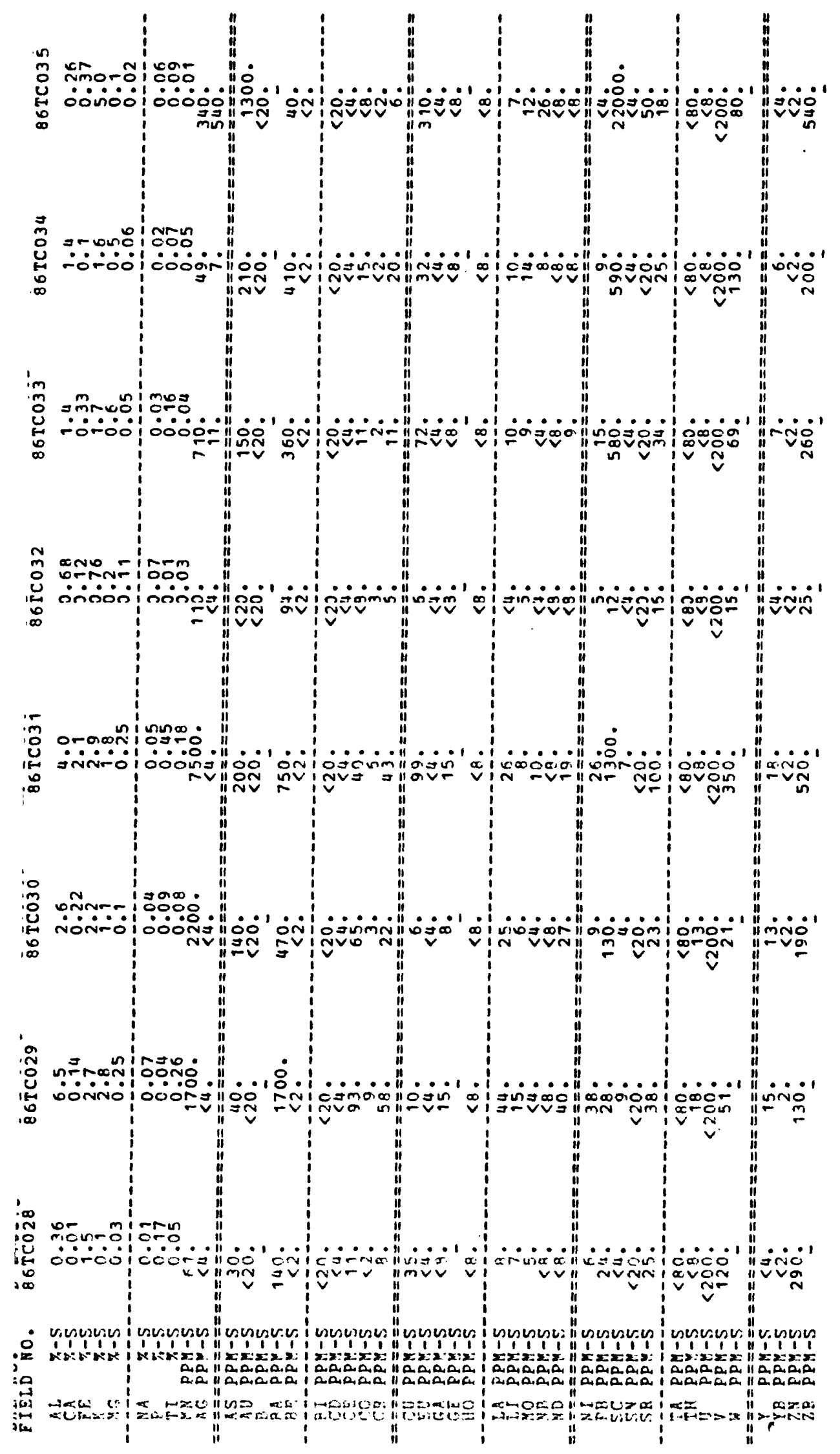




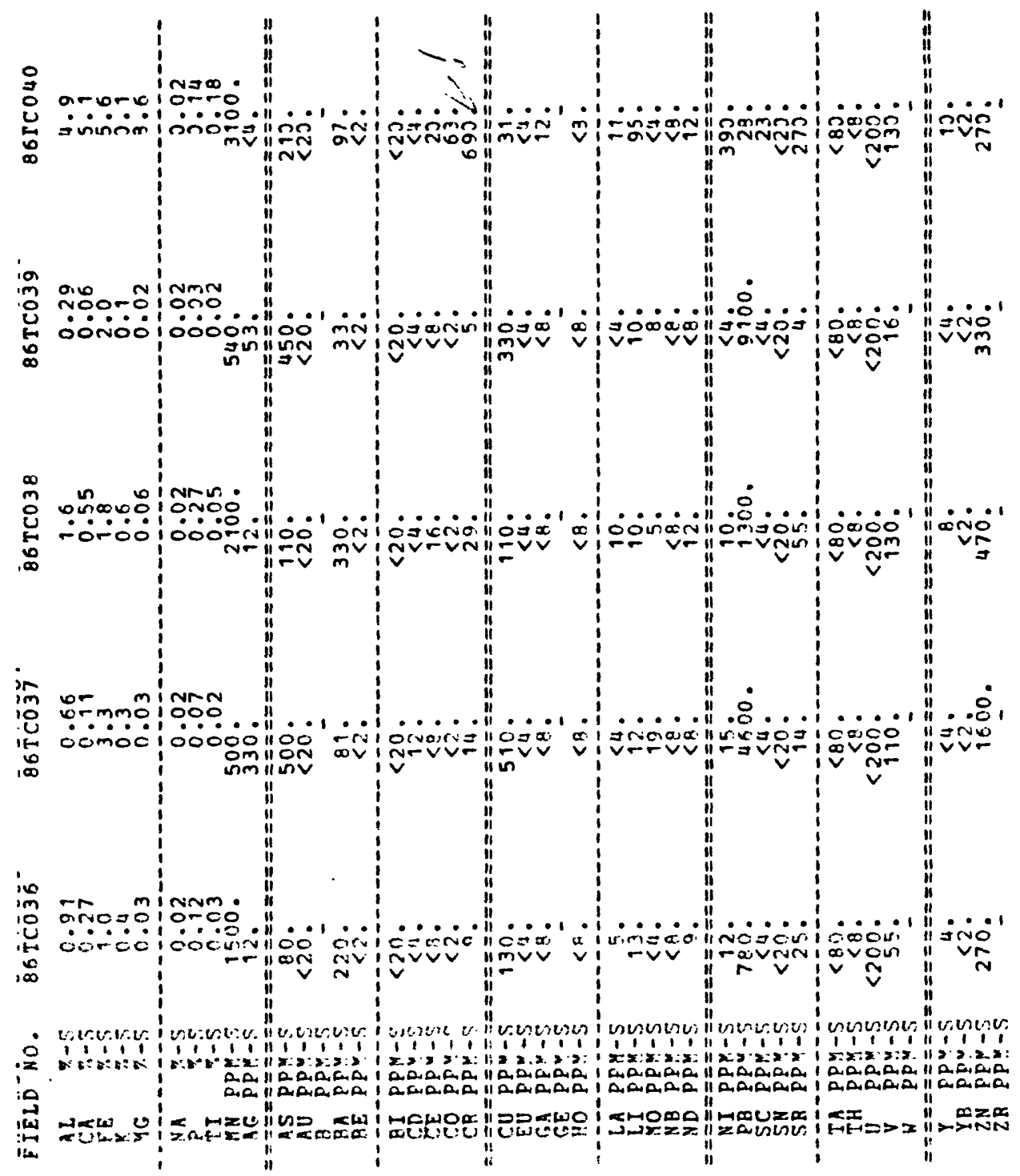




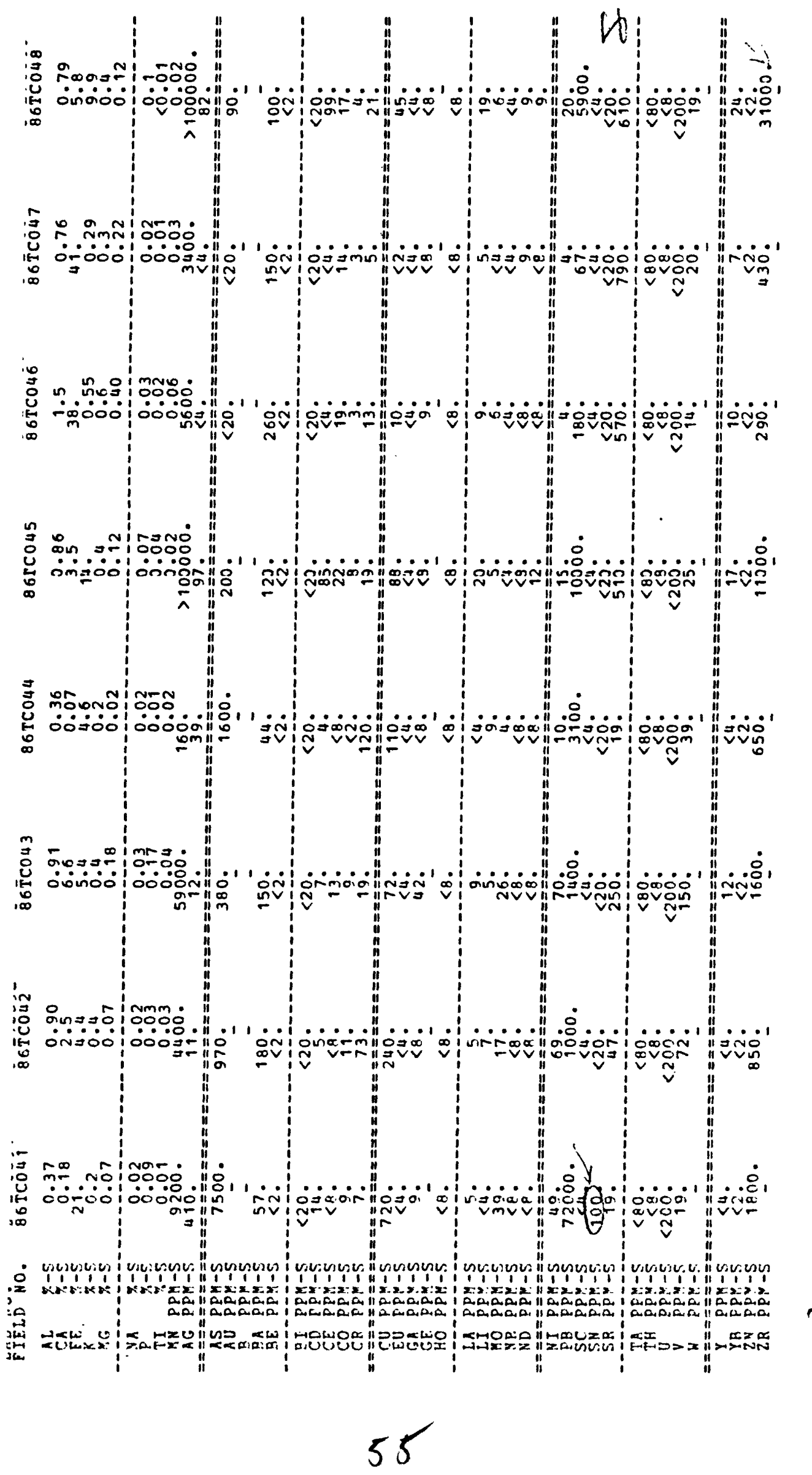




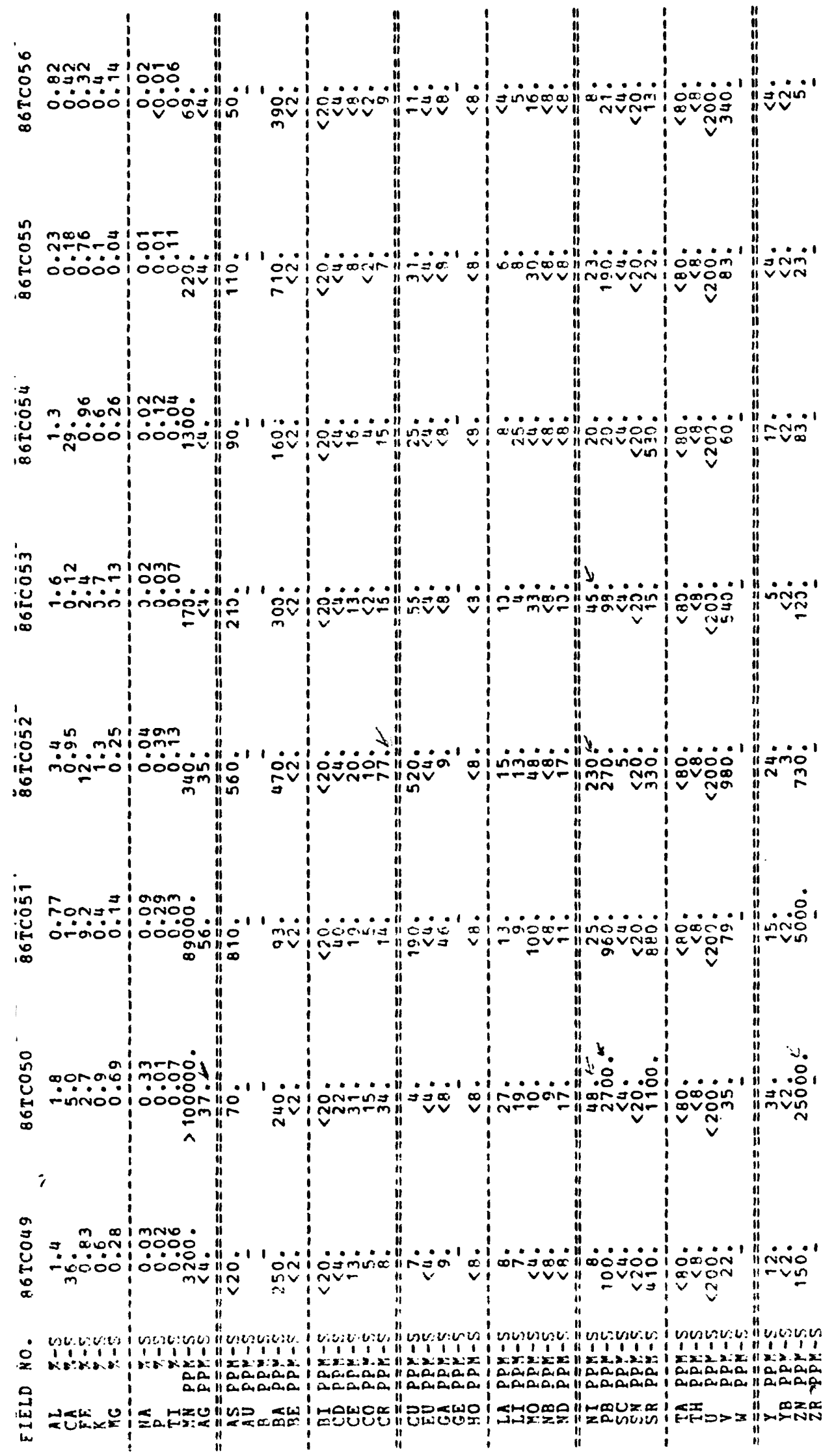




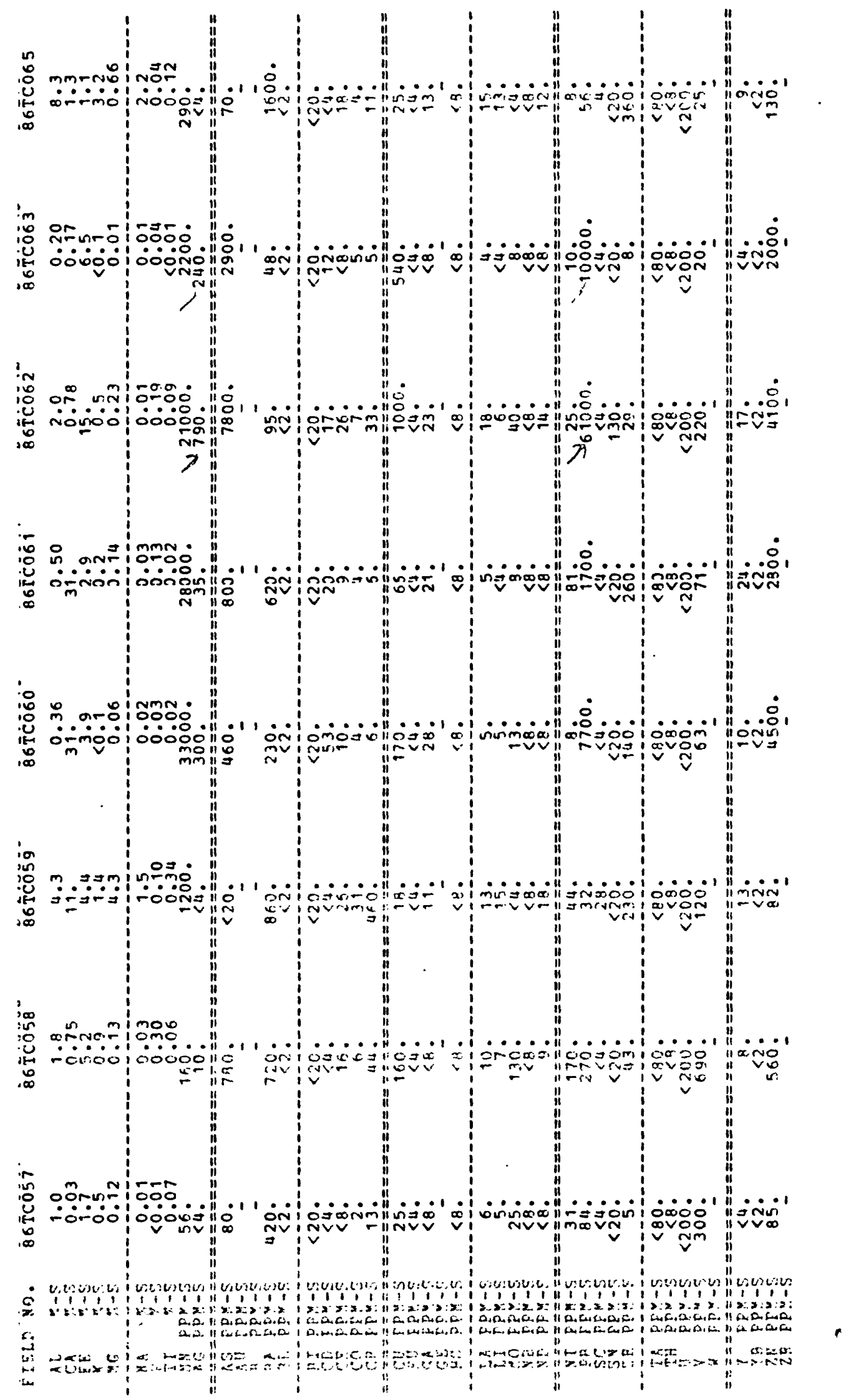




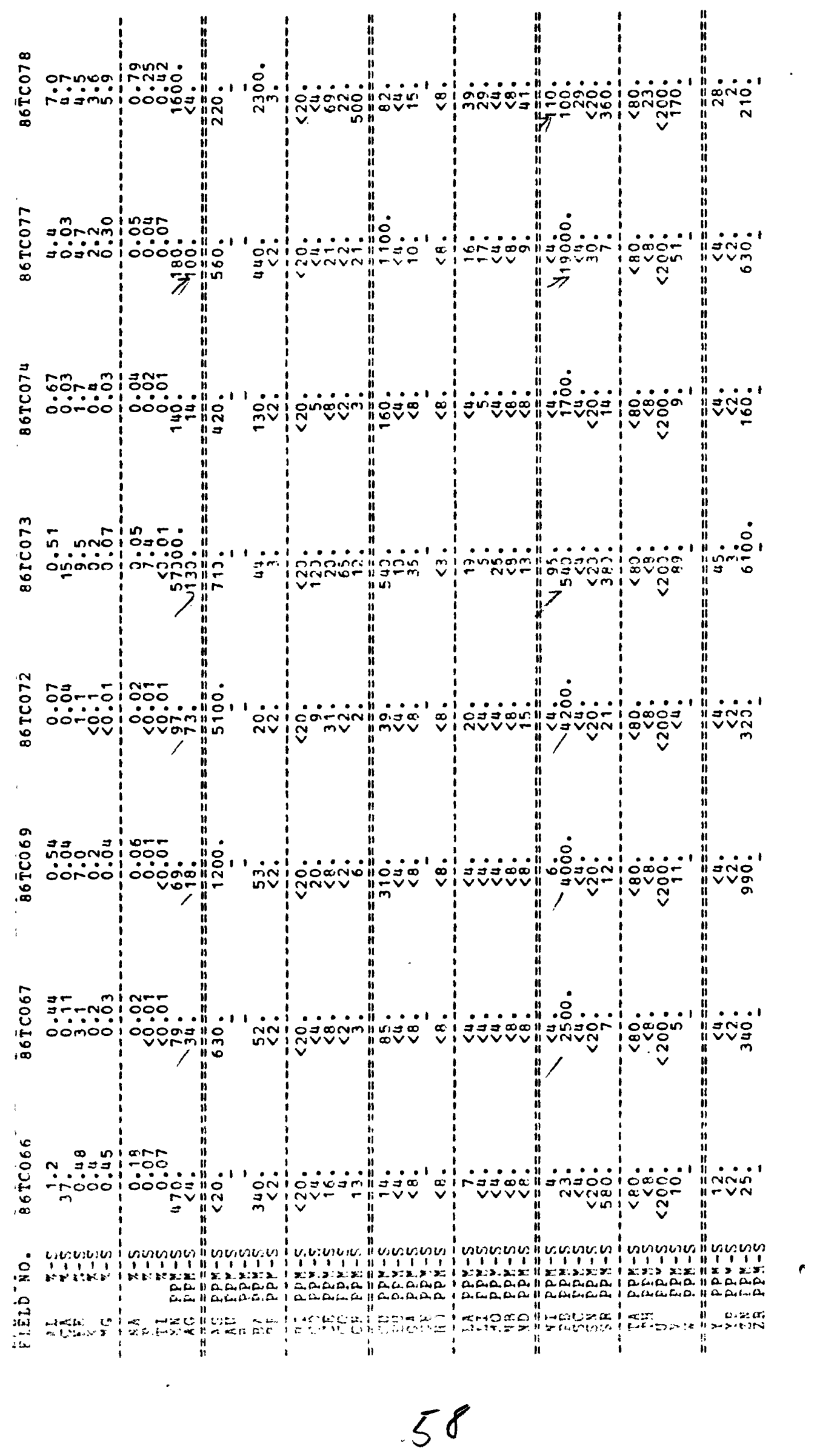




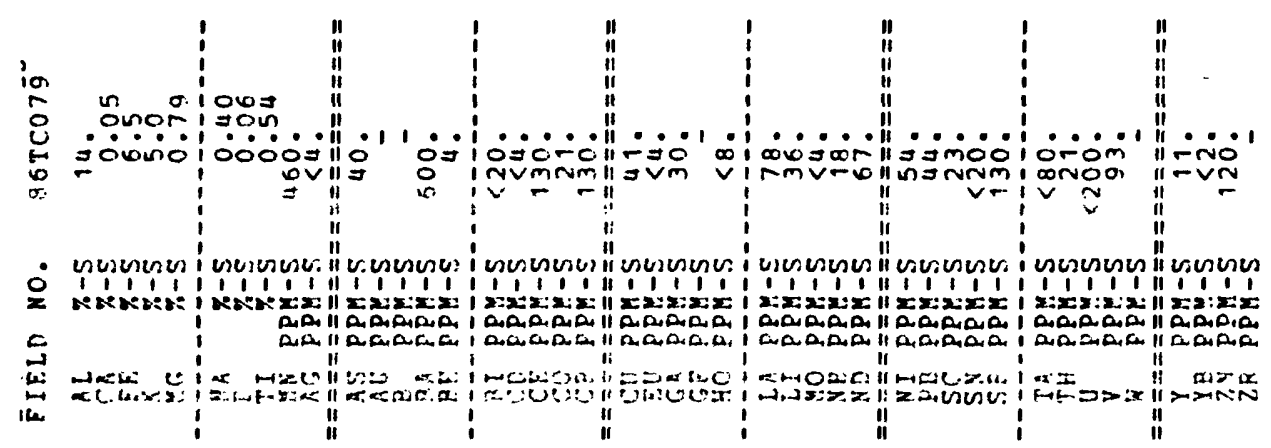




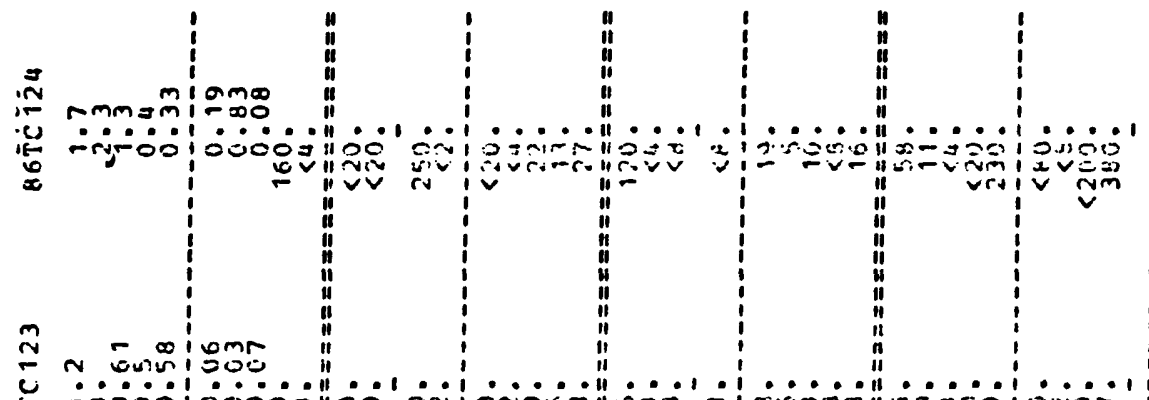

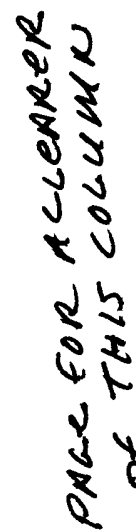

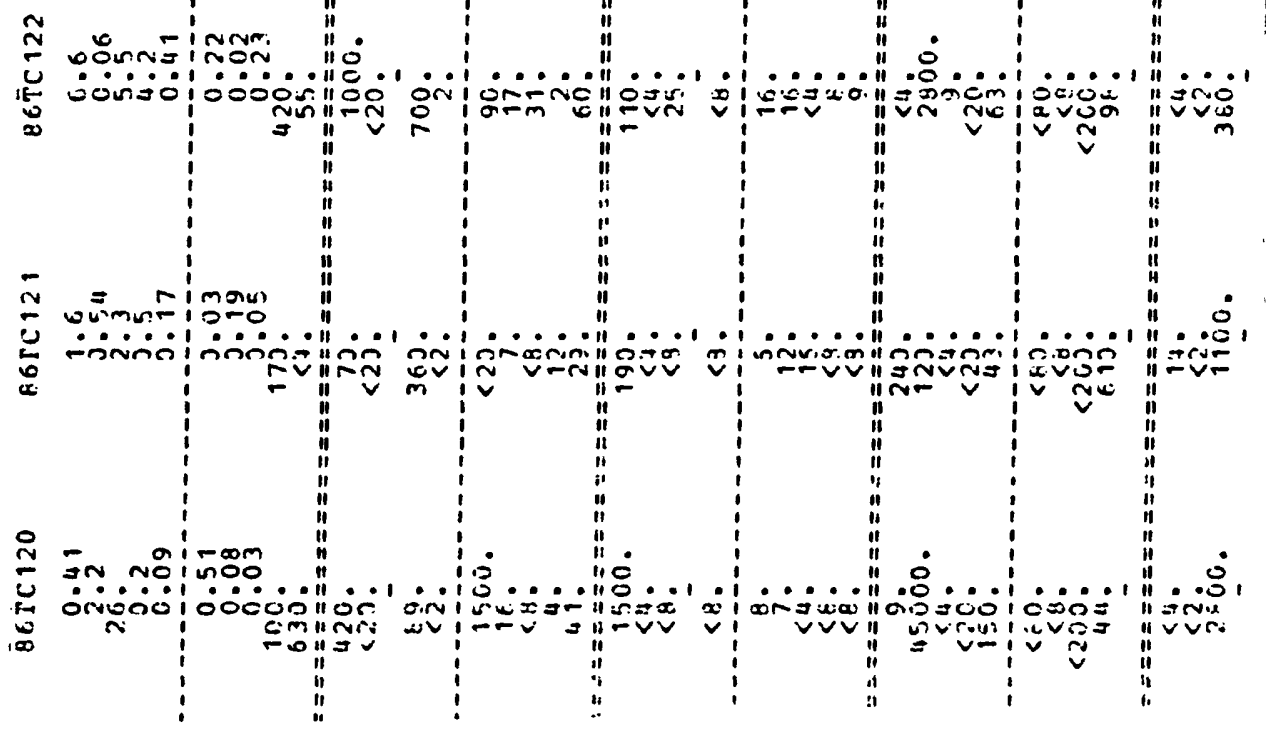

2
5
5
0
2
2
2
0
5
5
2

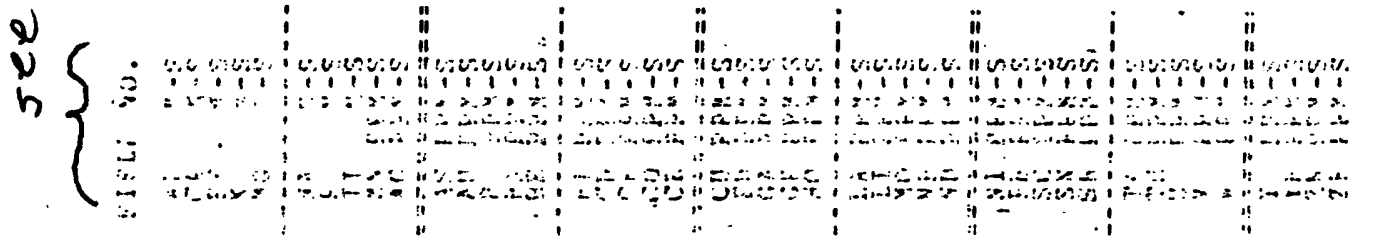




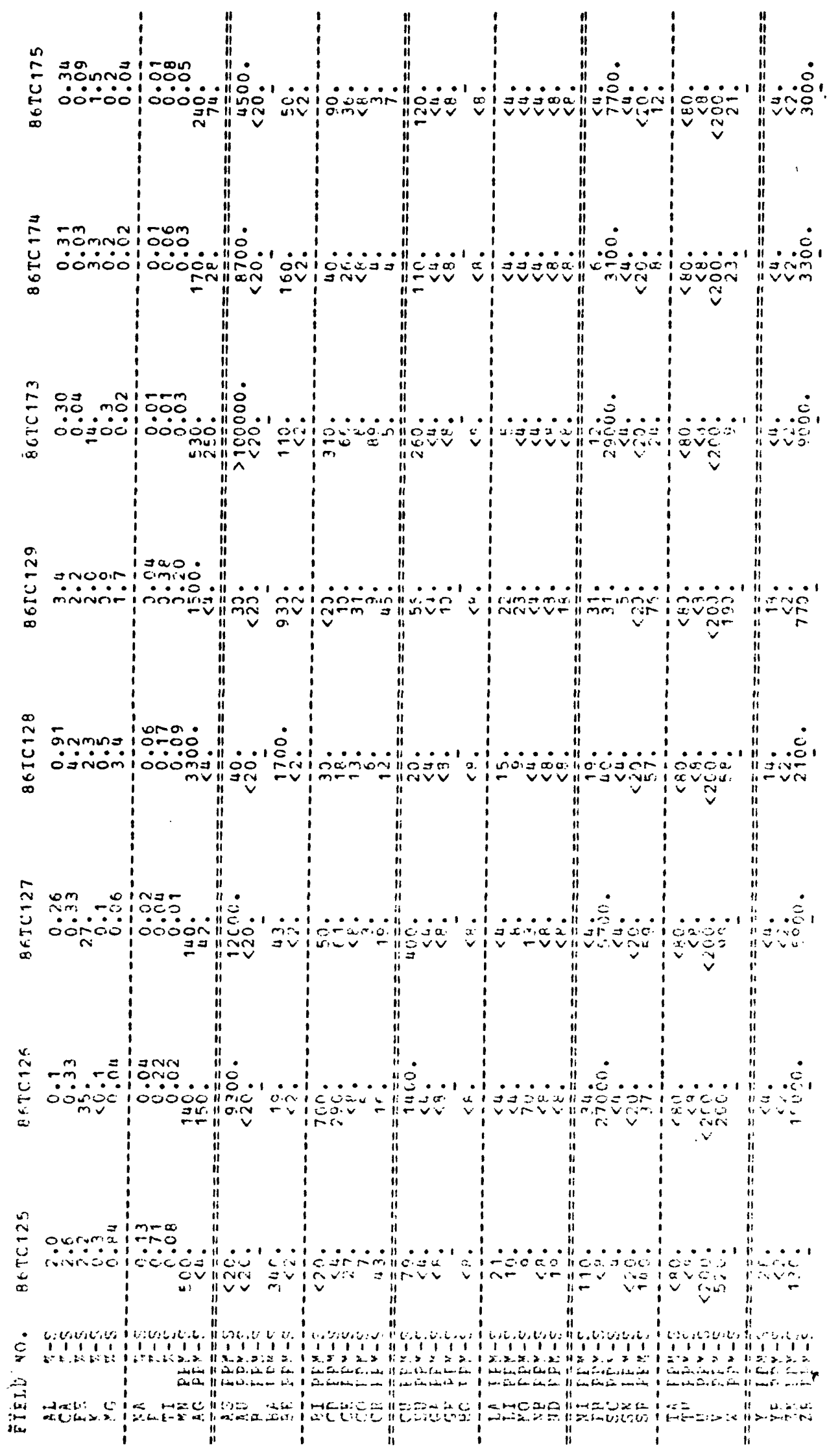




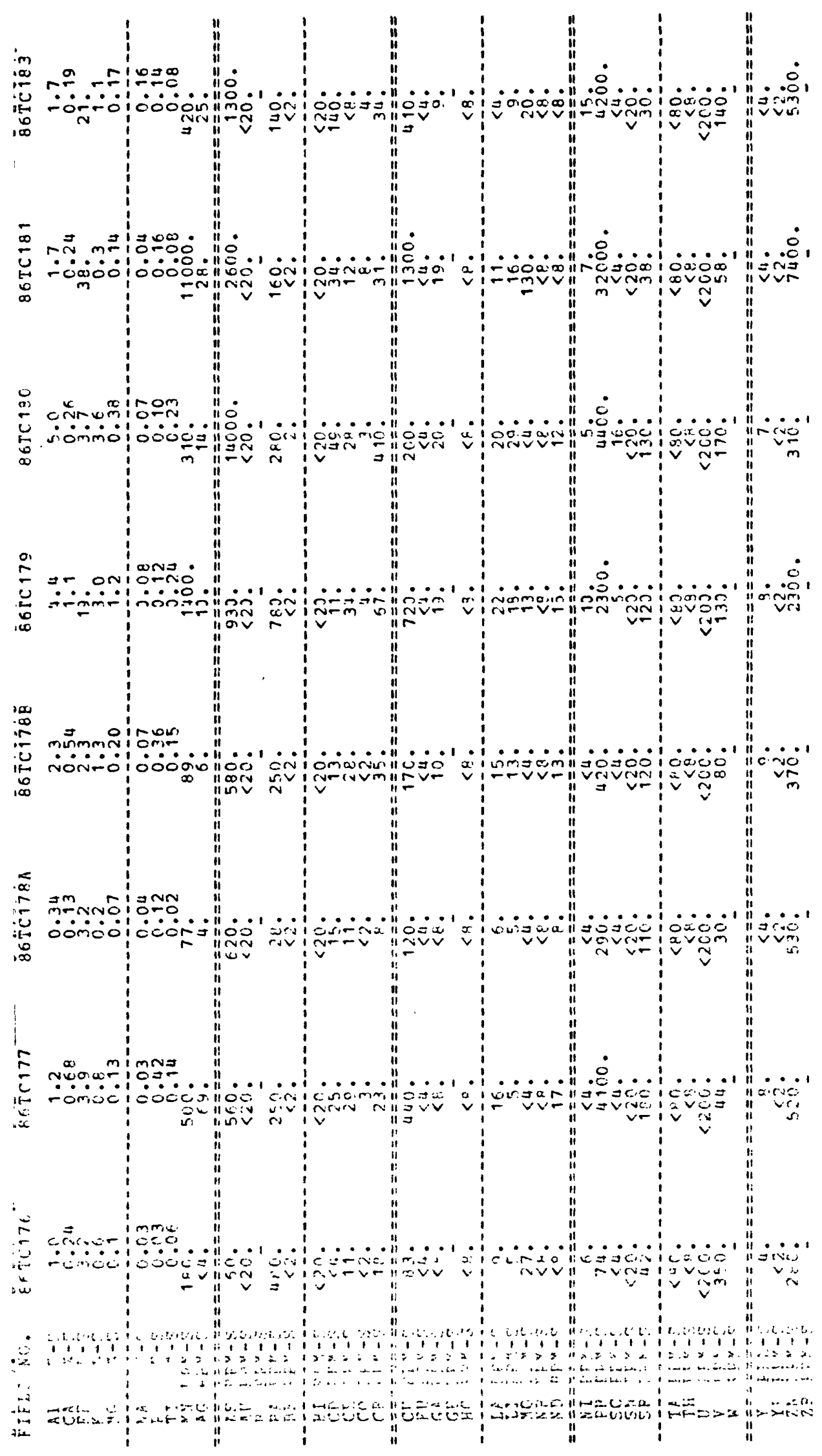

$6^{2}$ 


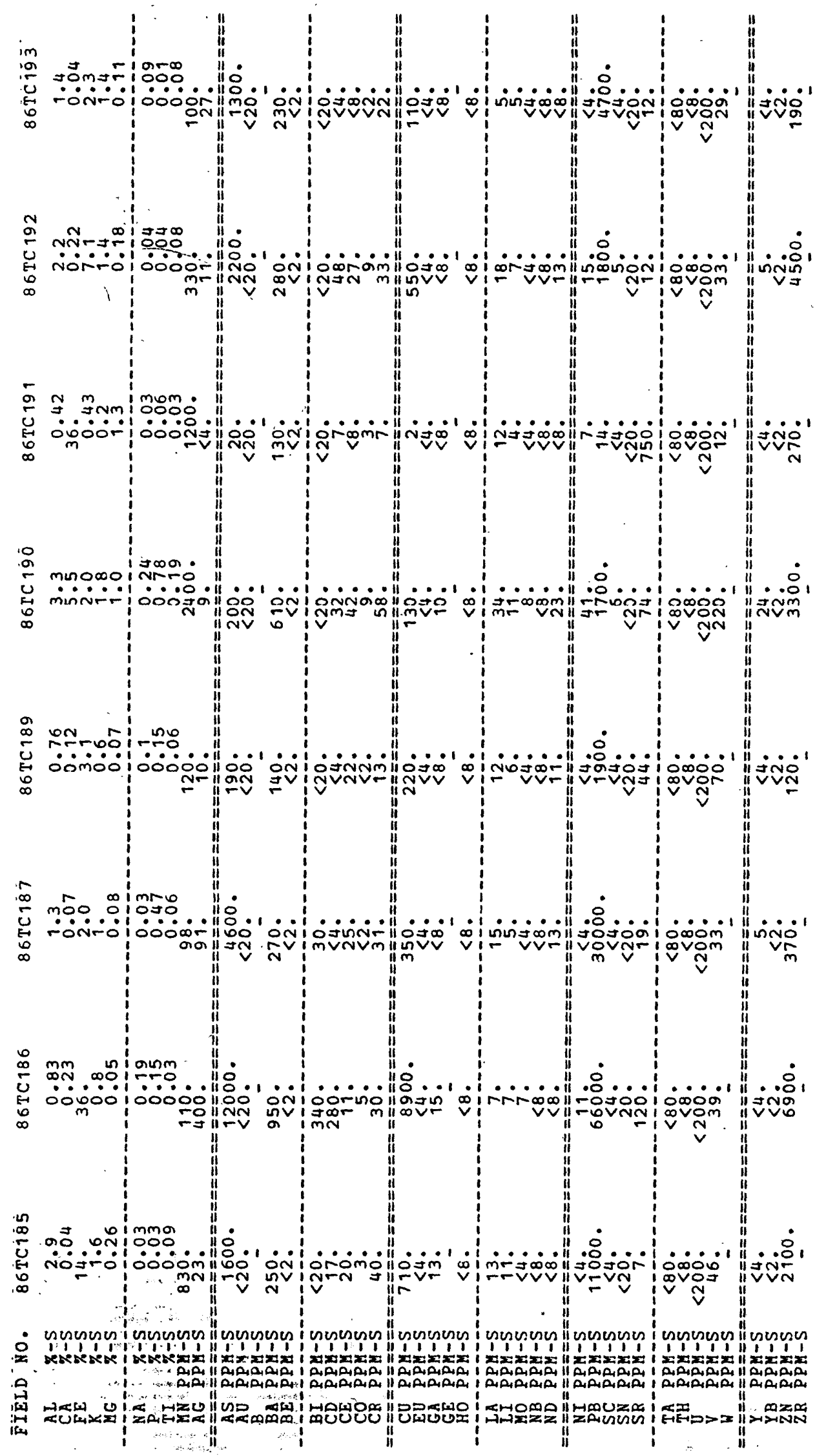




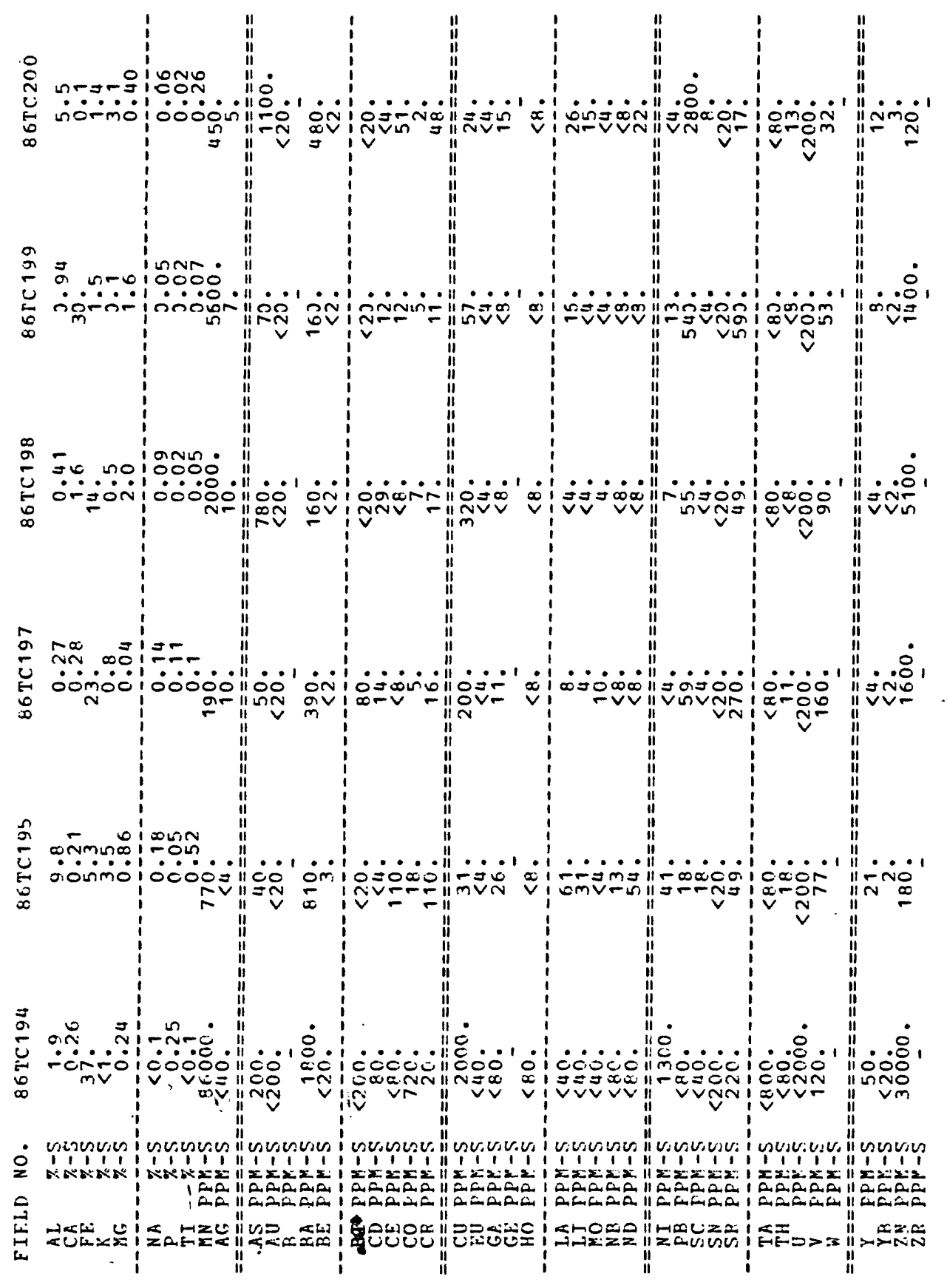




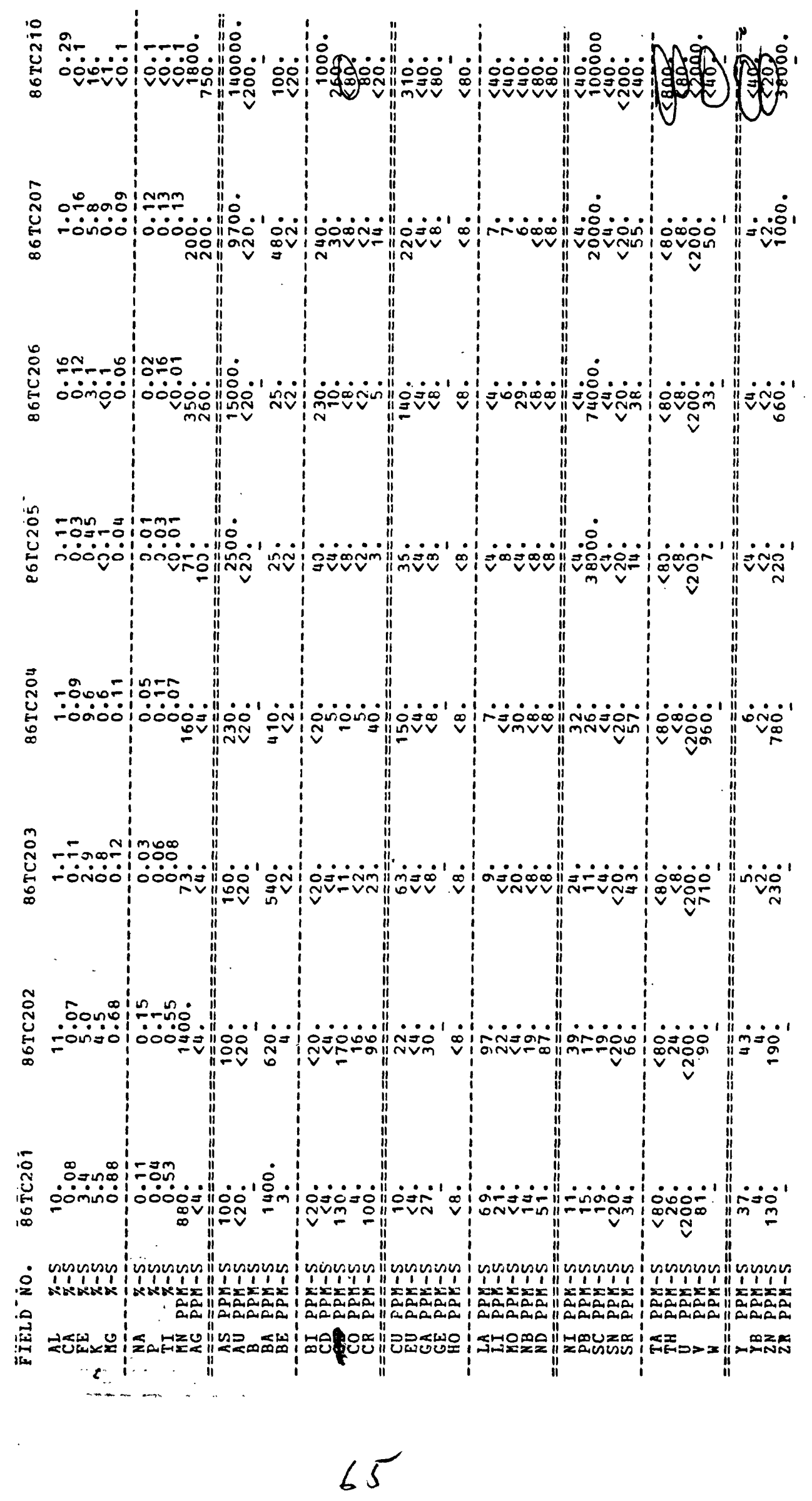




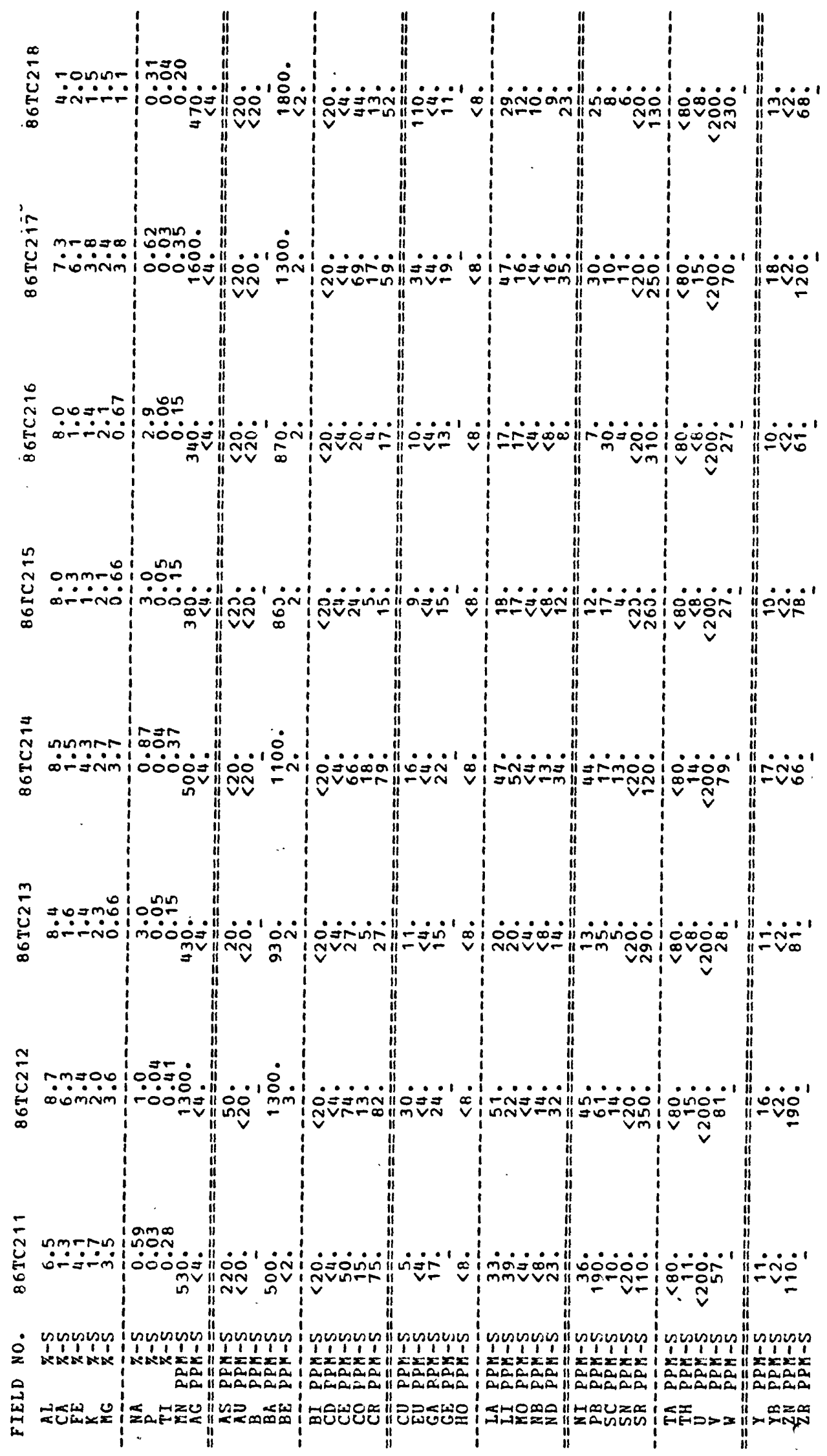




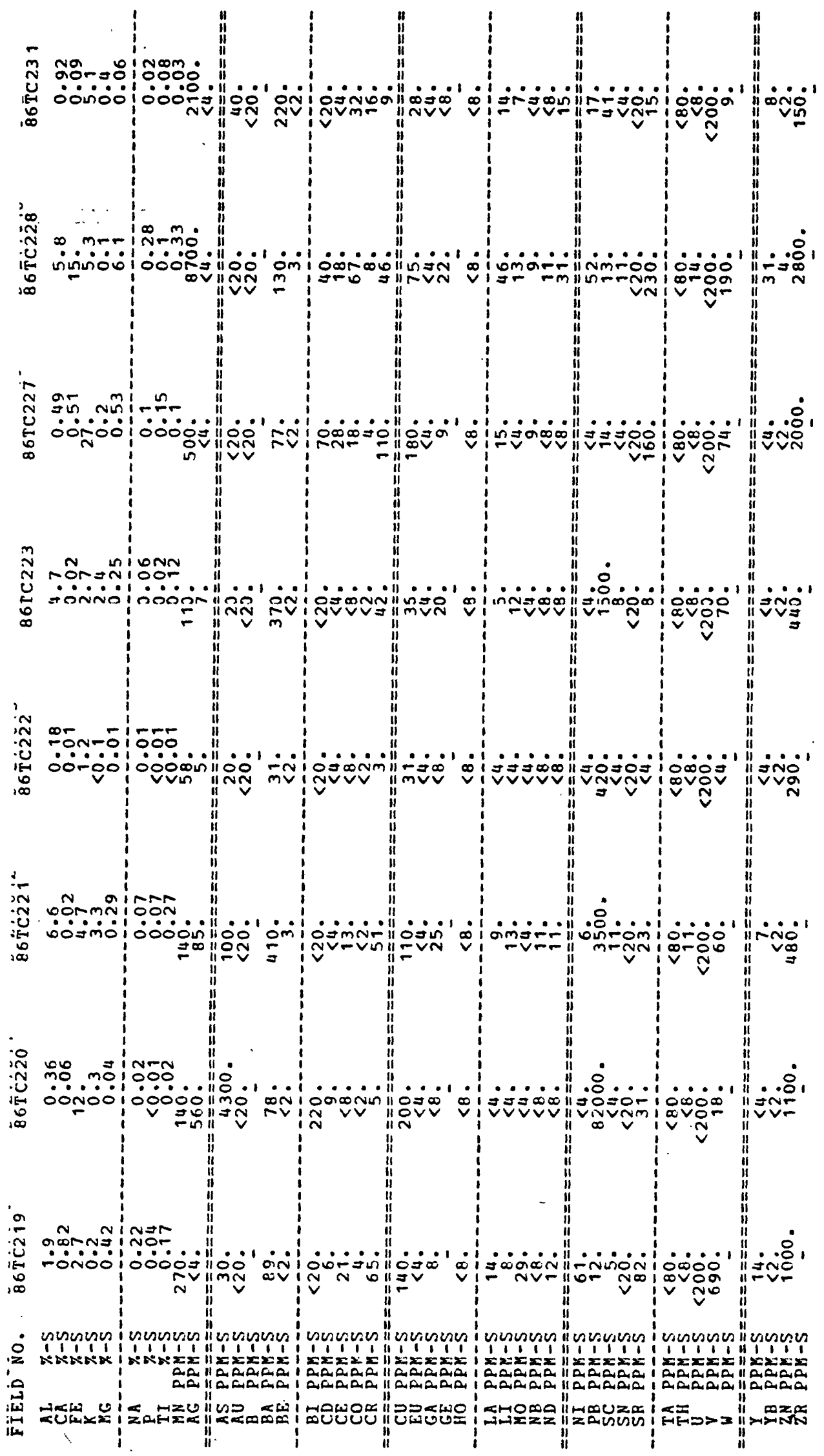




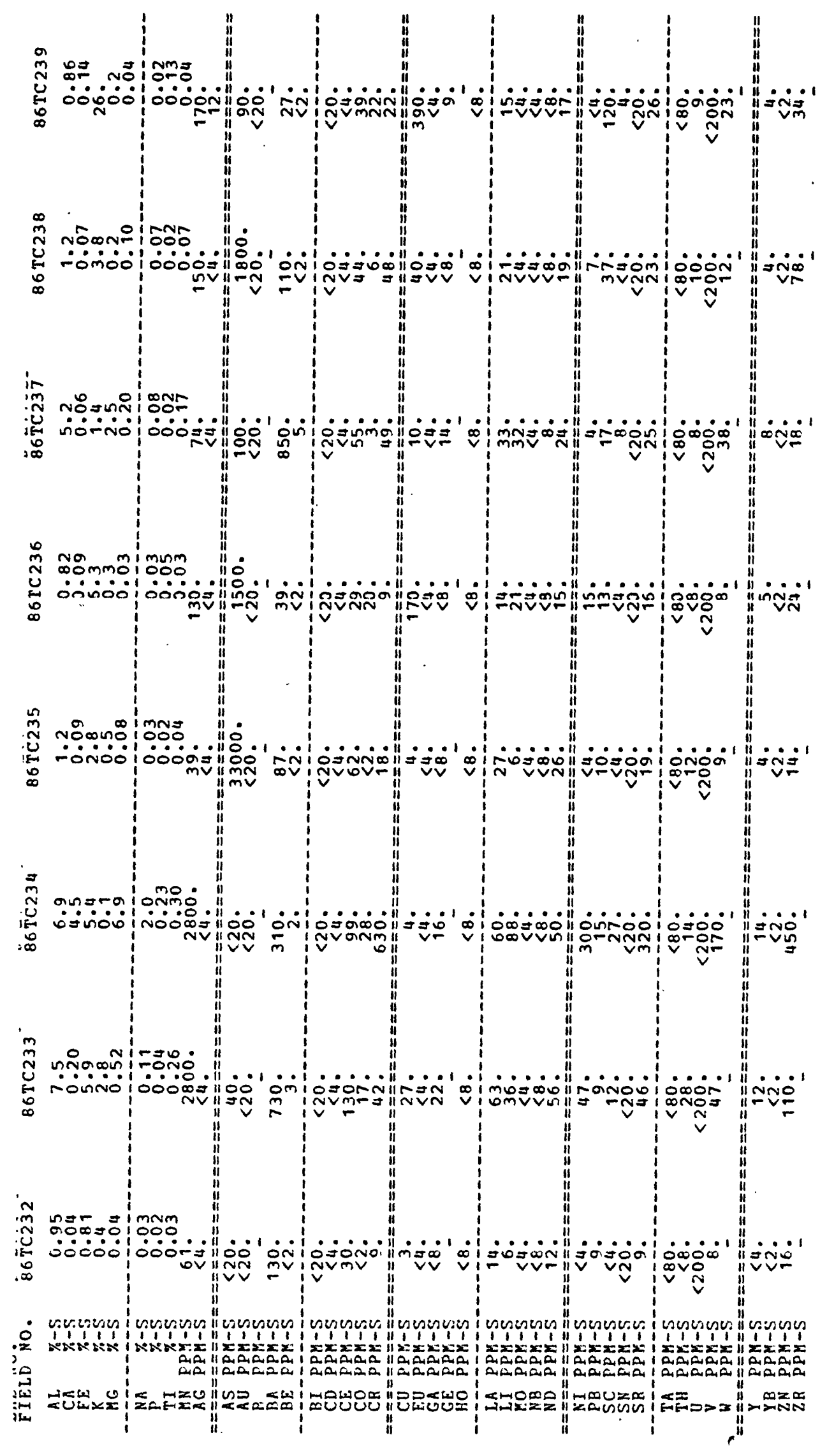




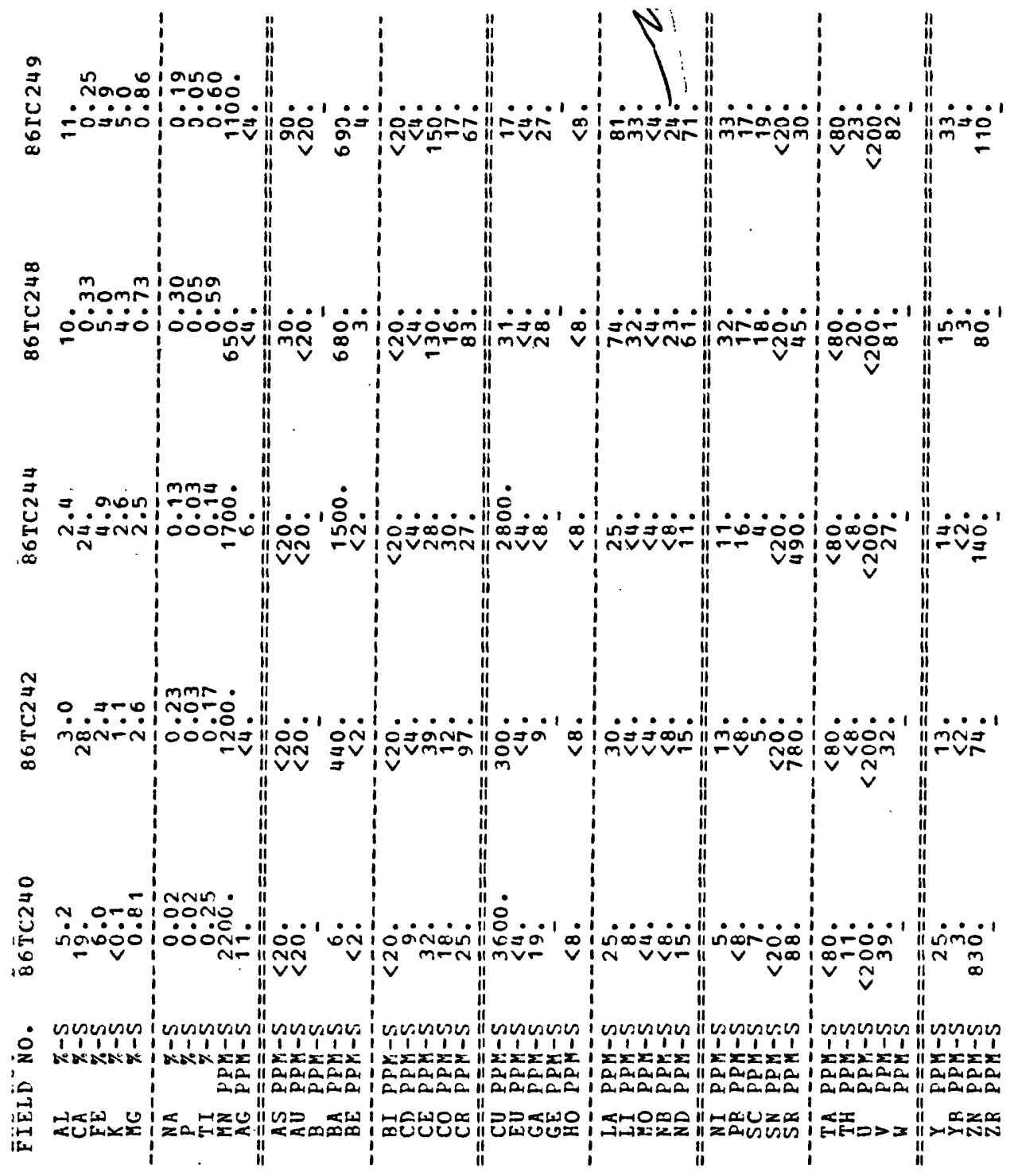




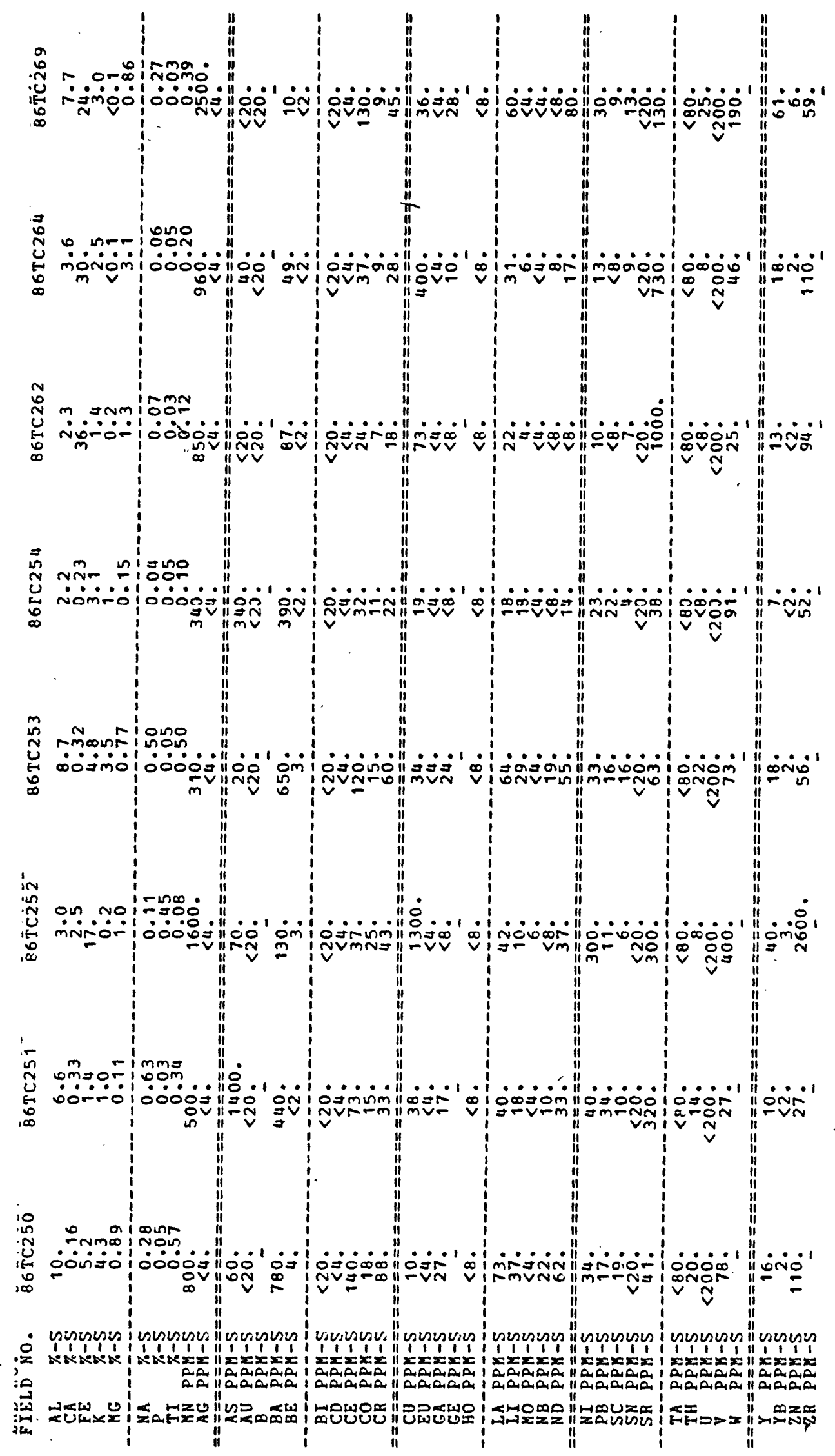




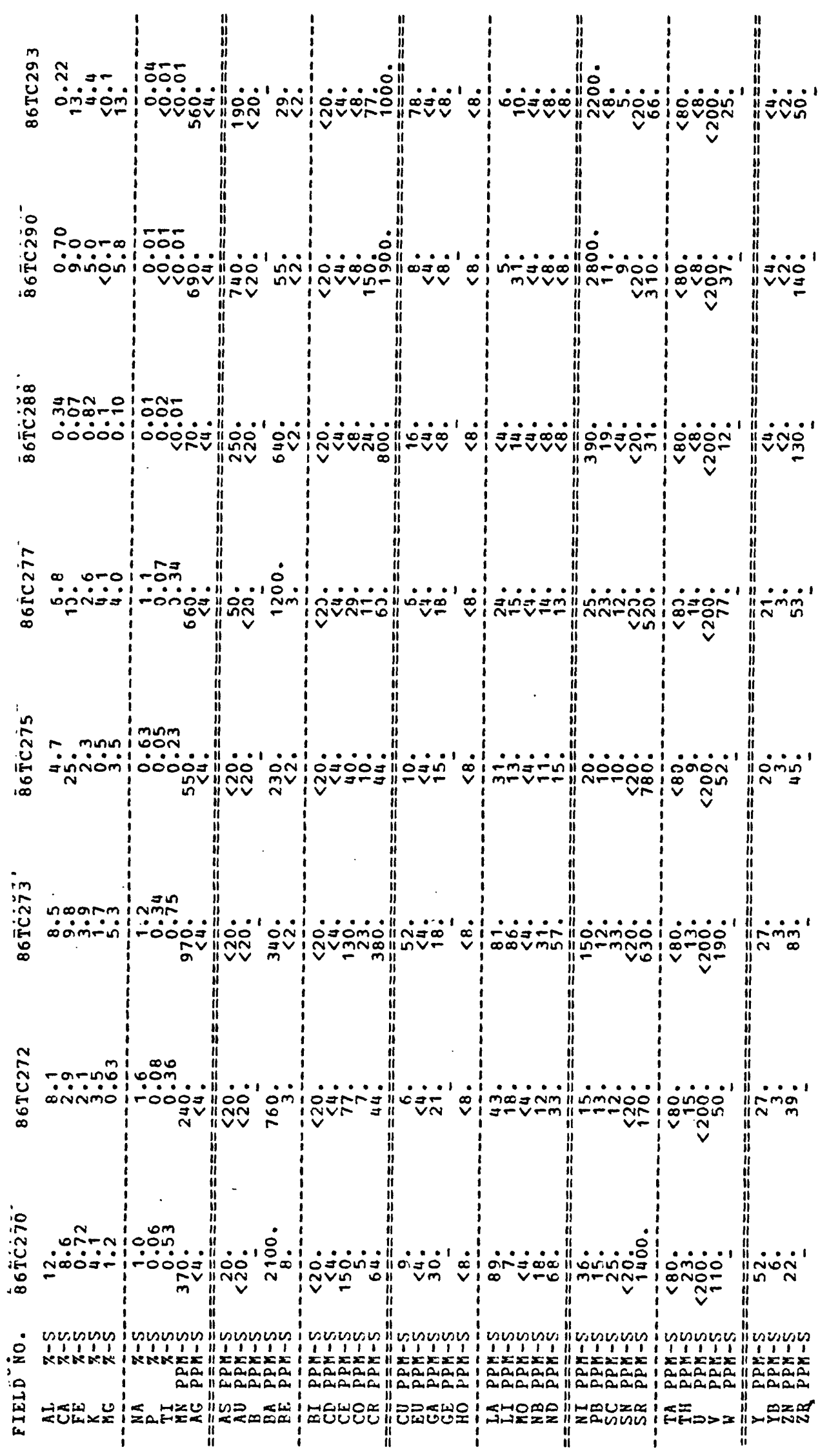




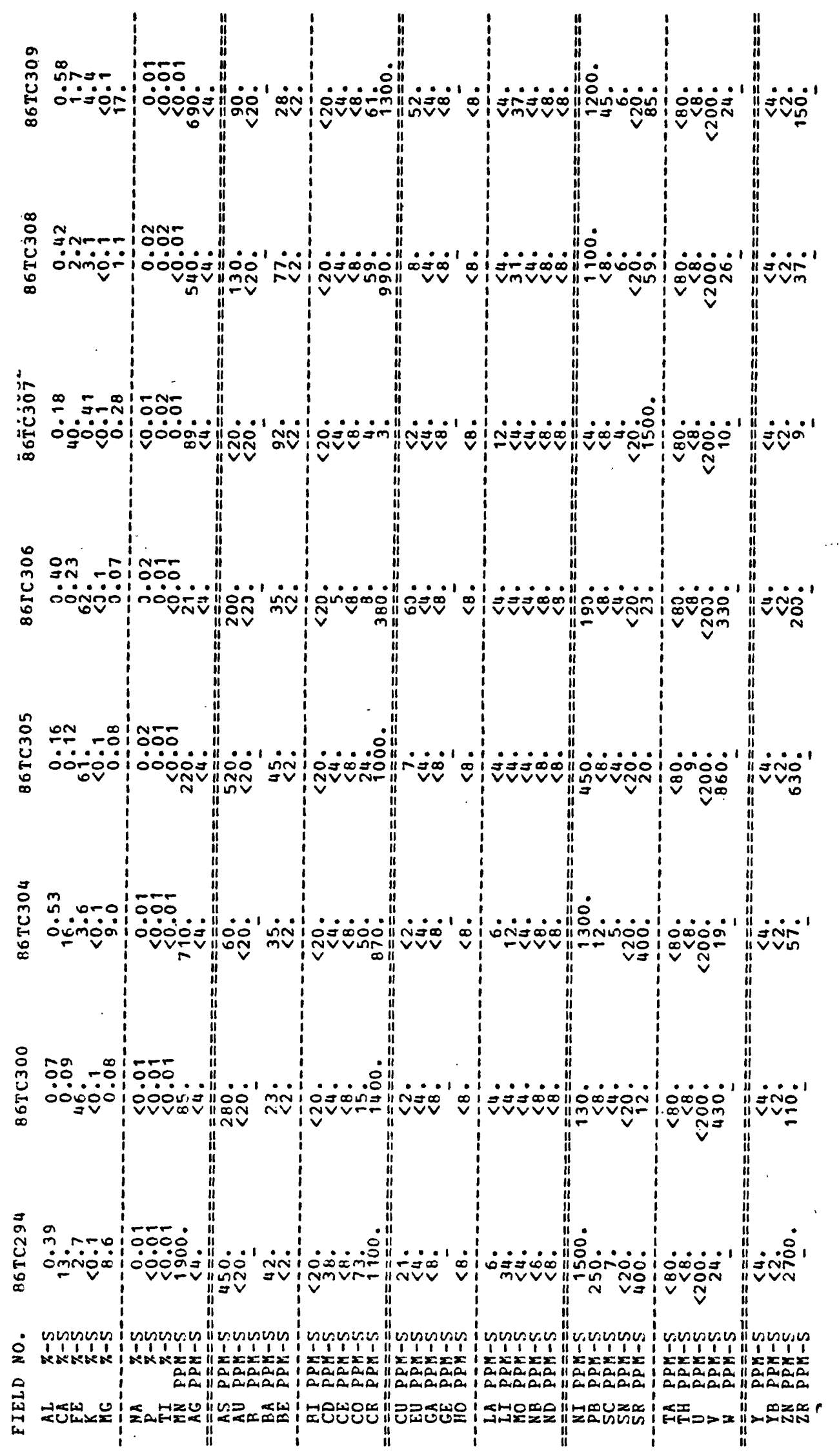




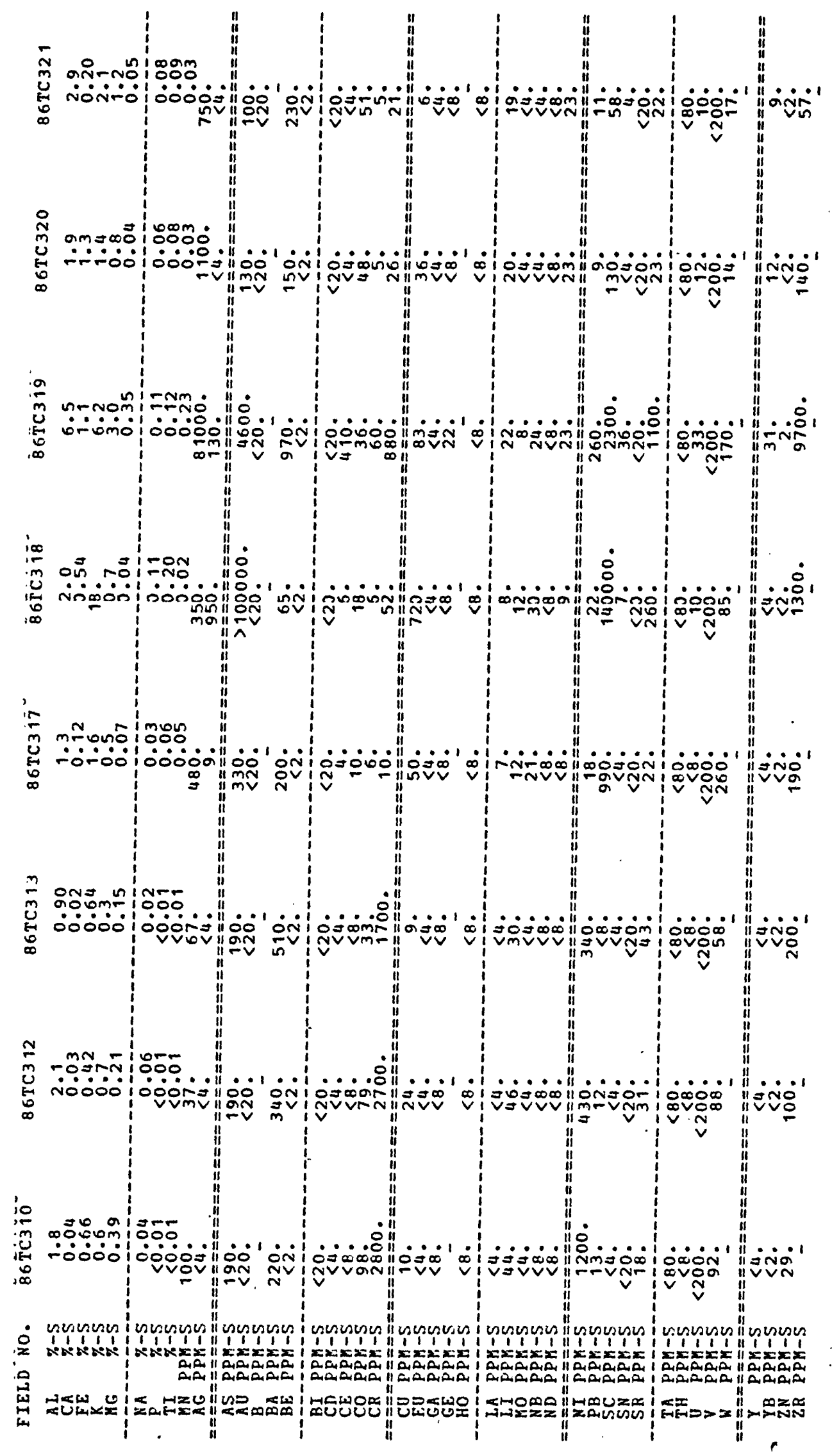




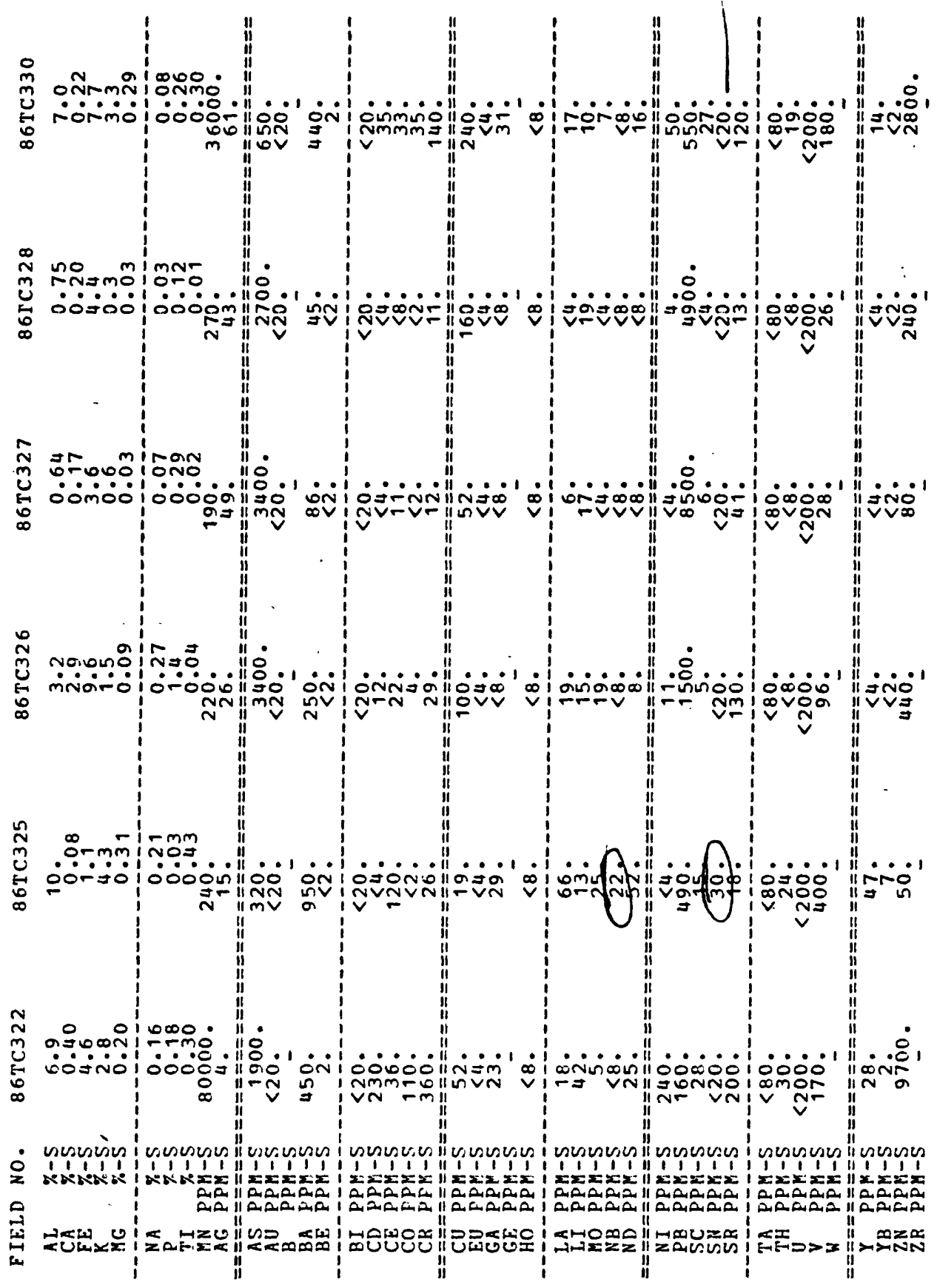




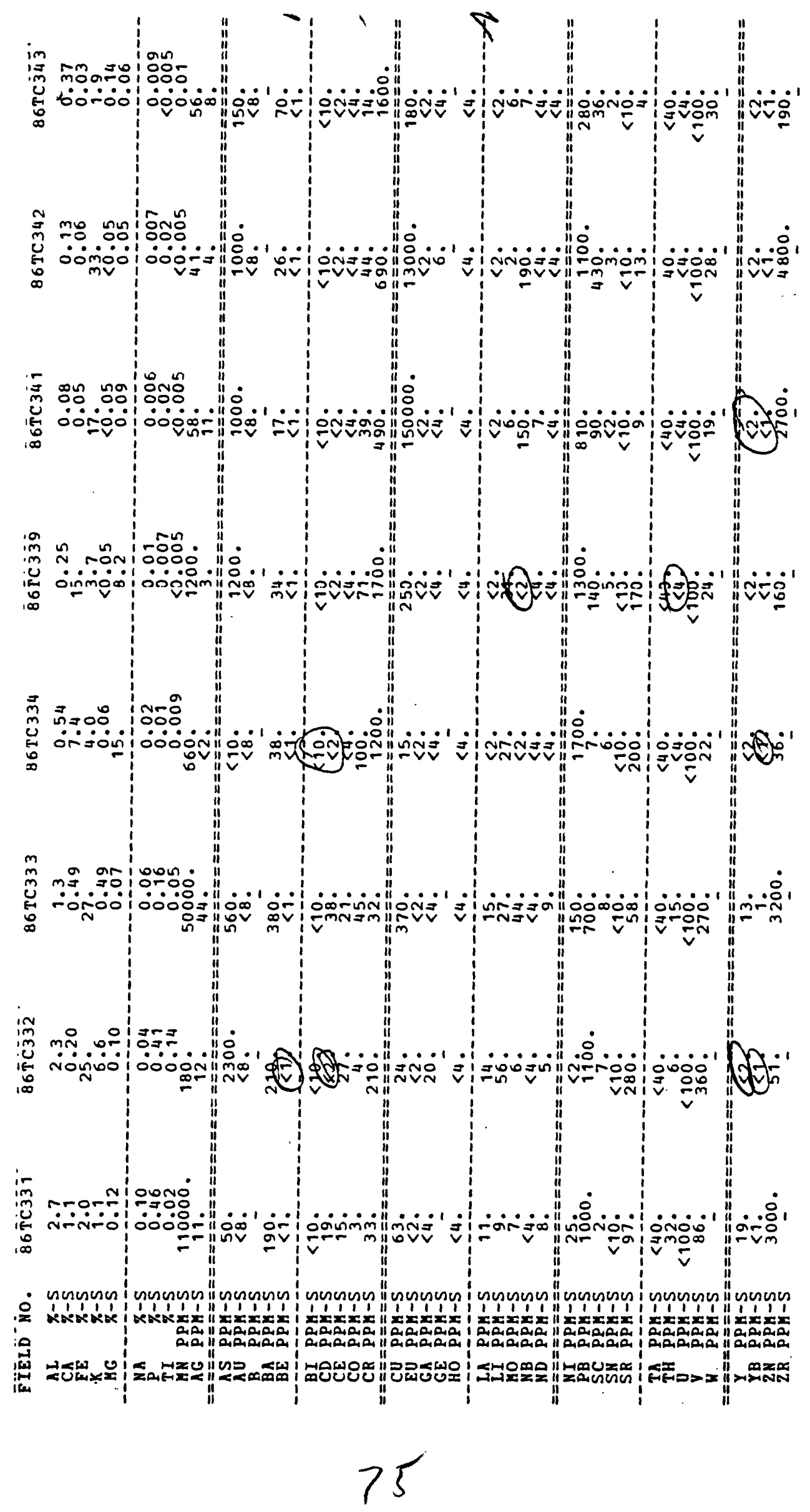




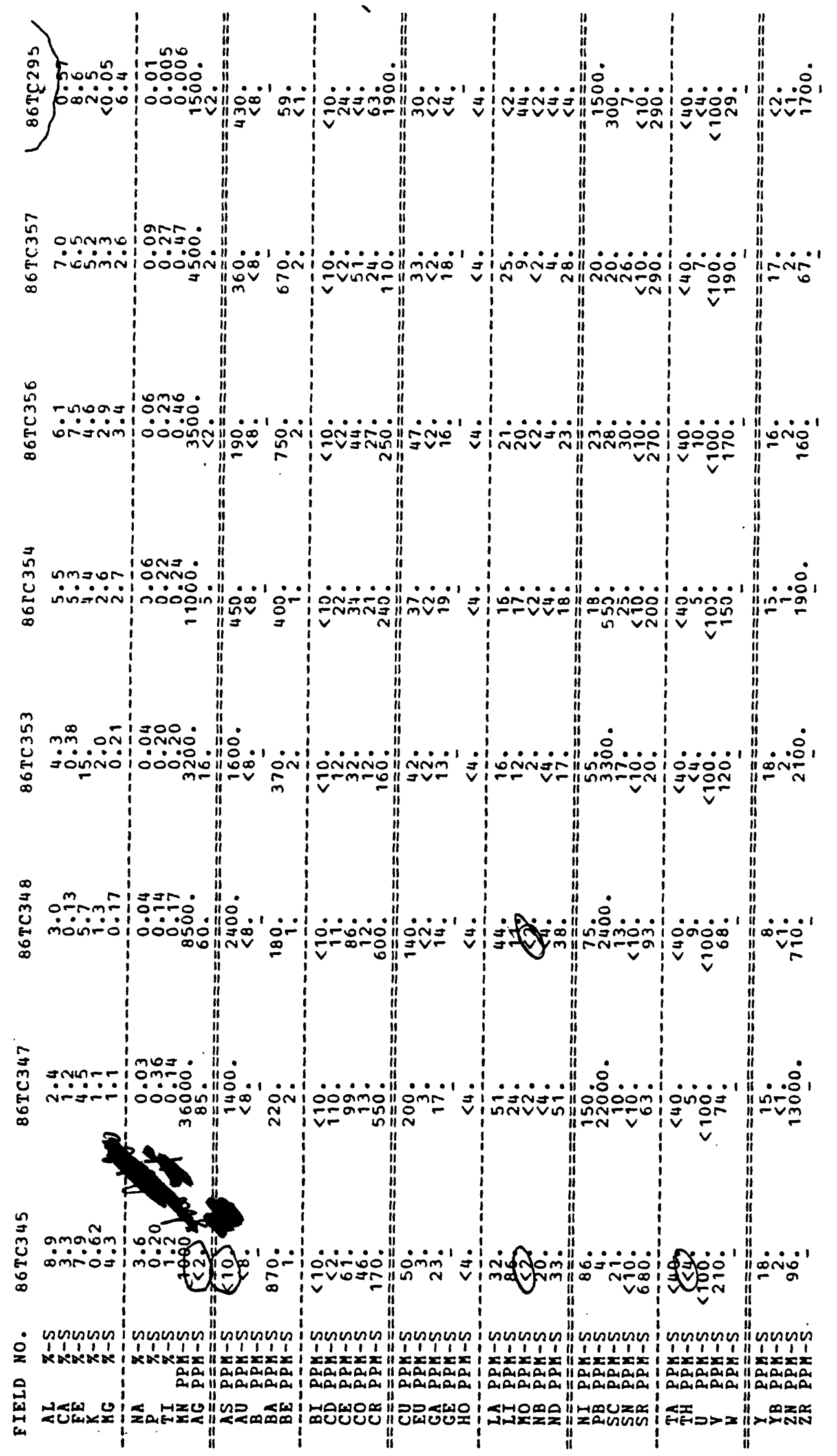




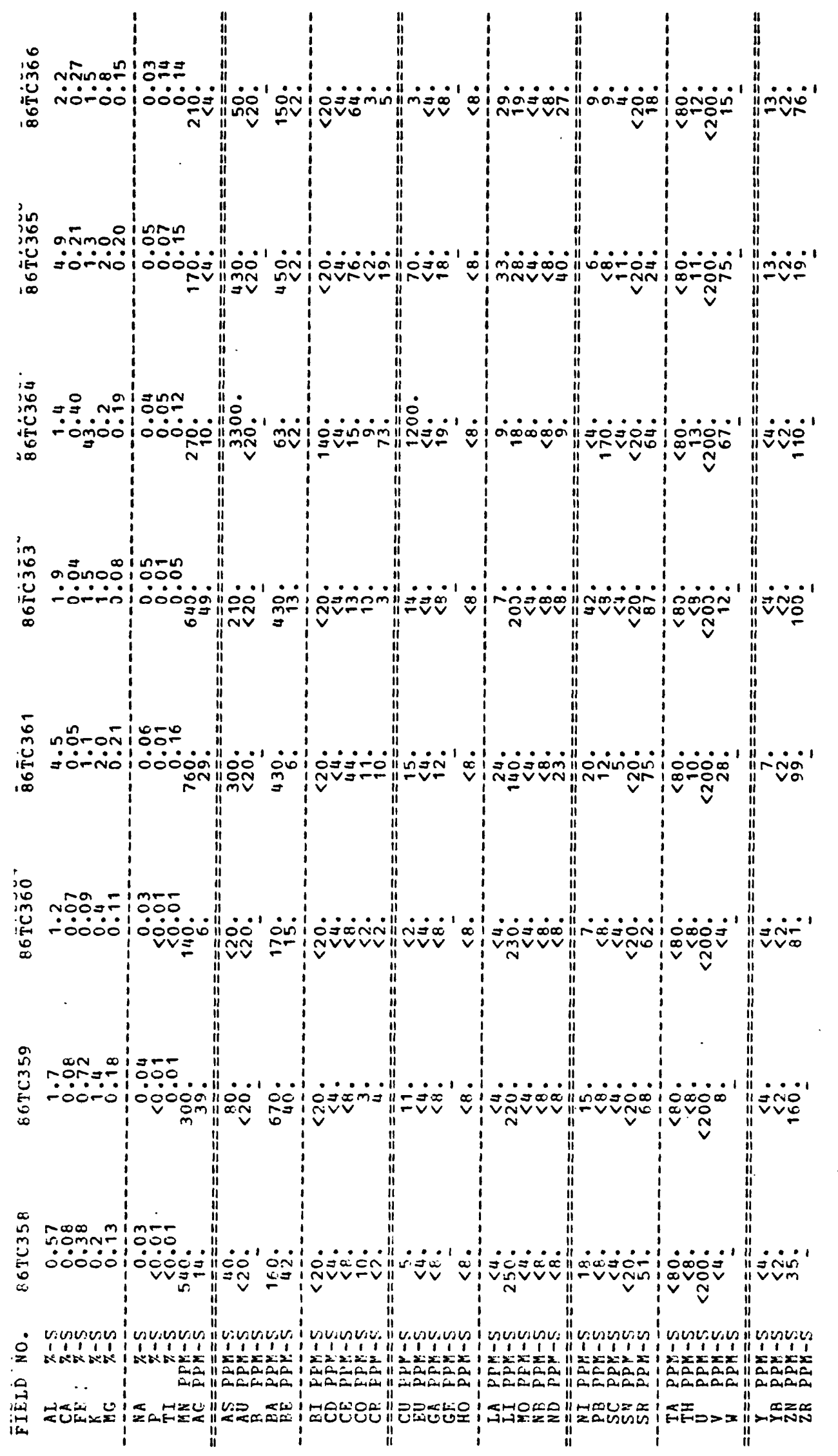




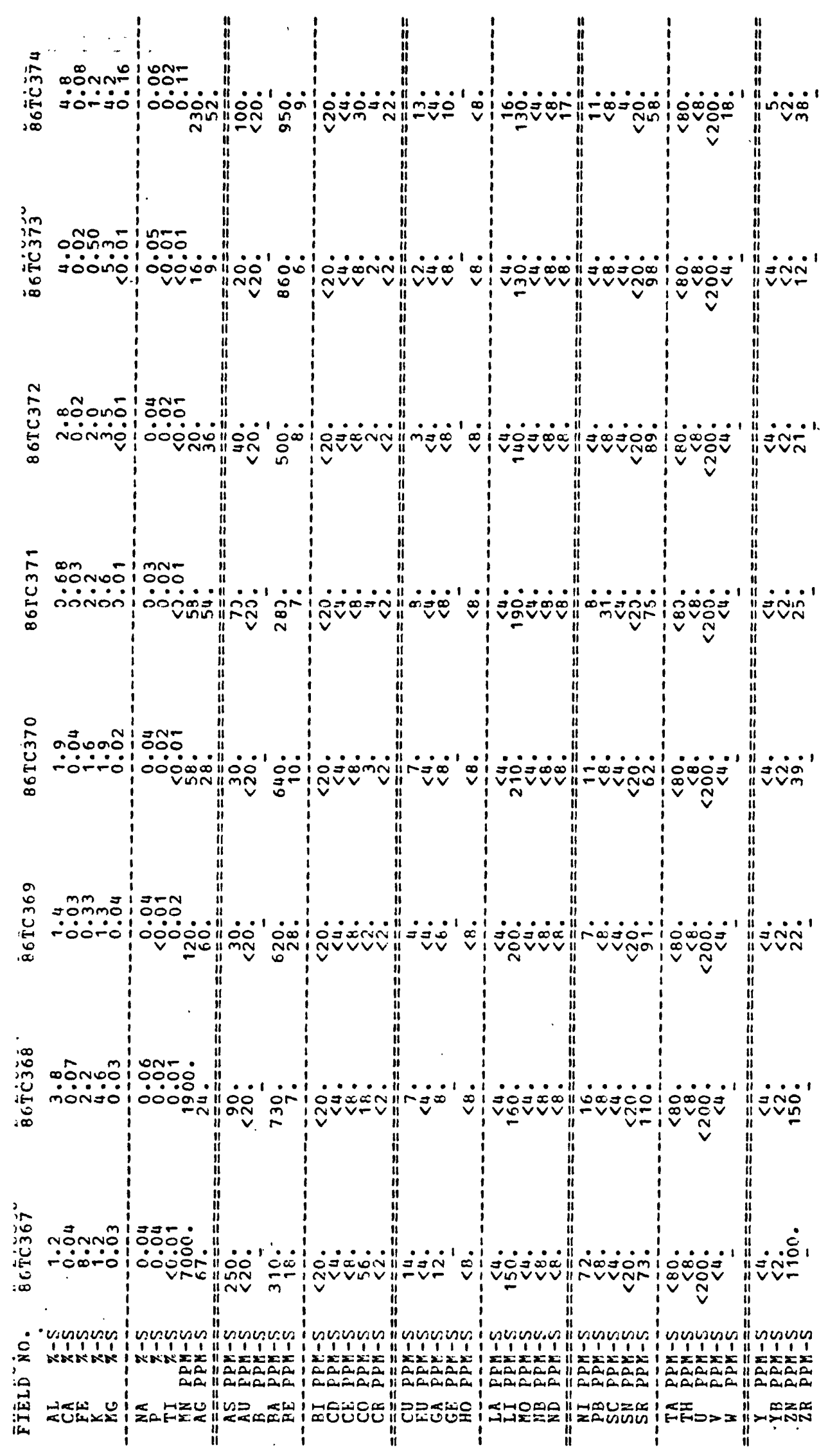




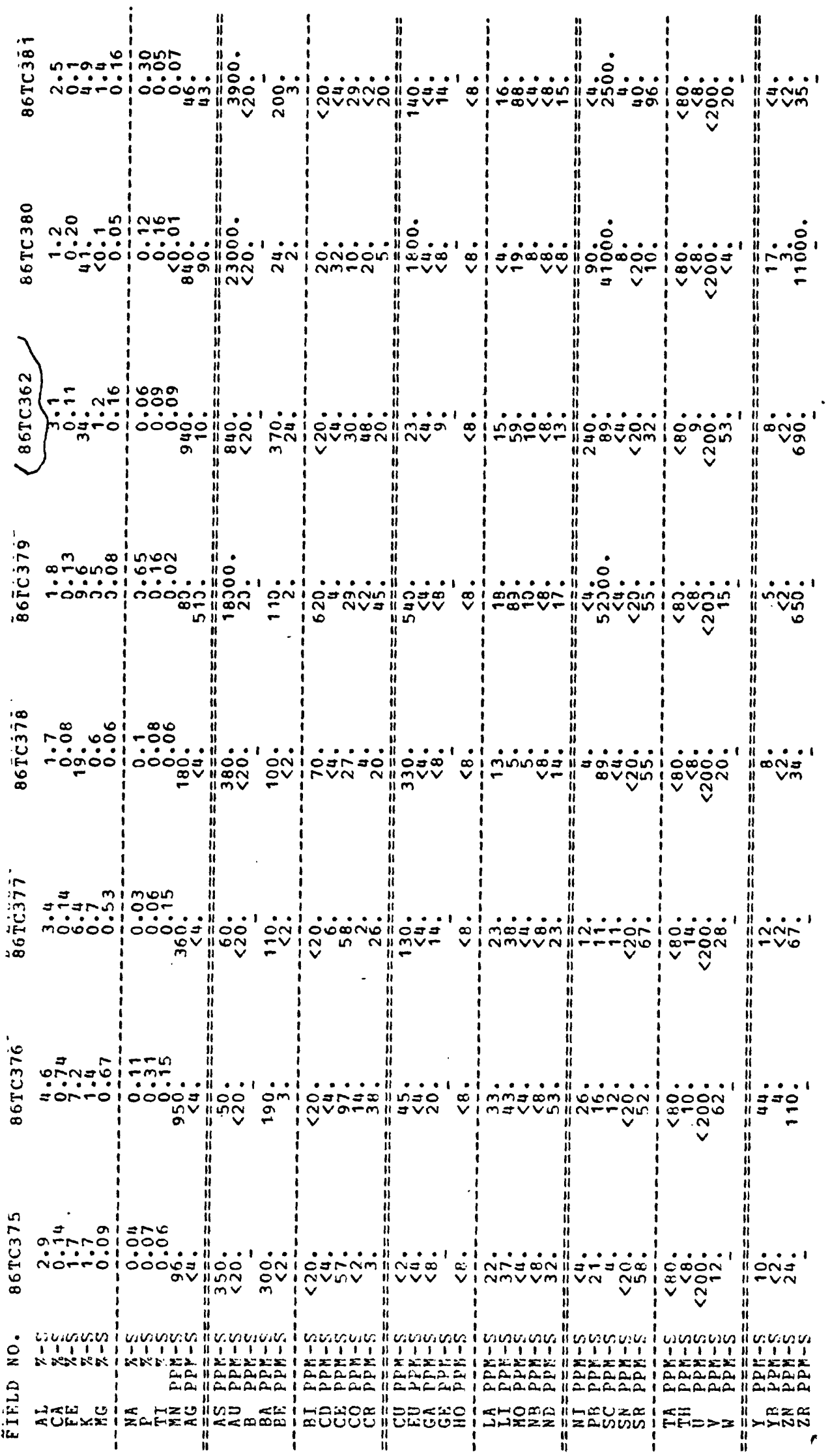




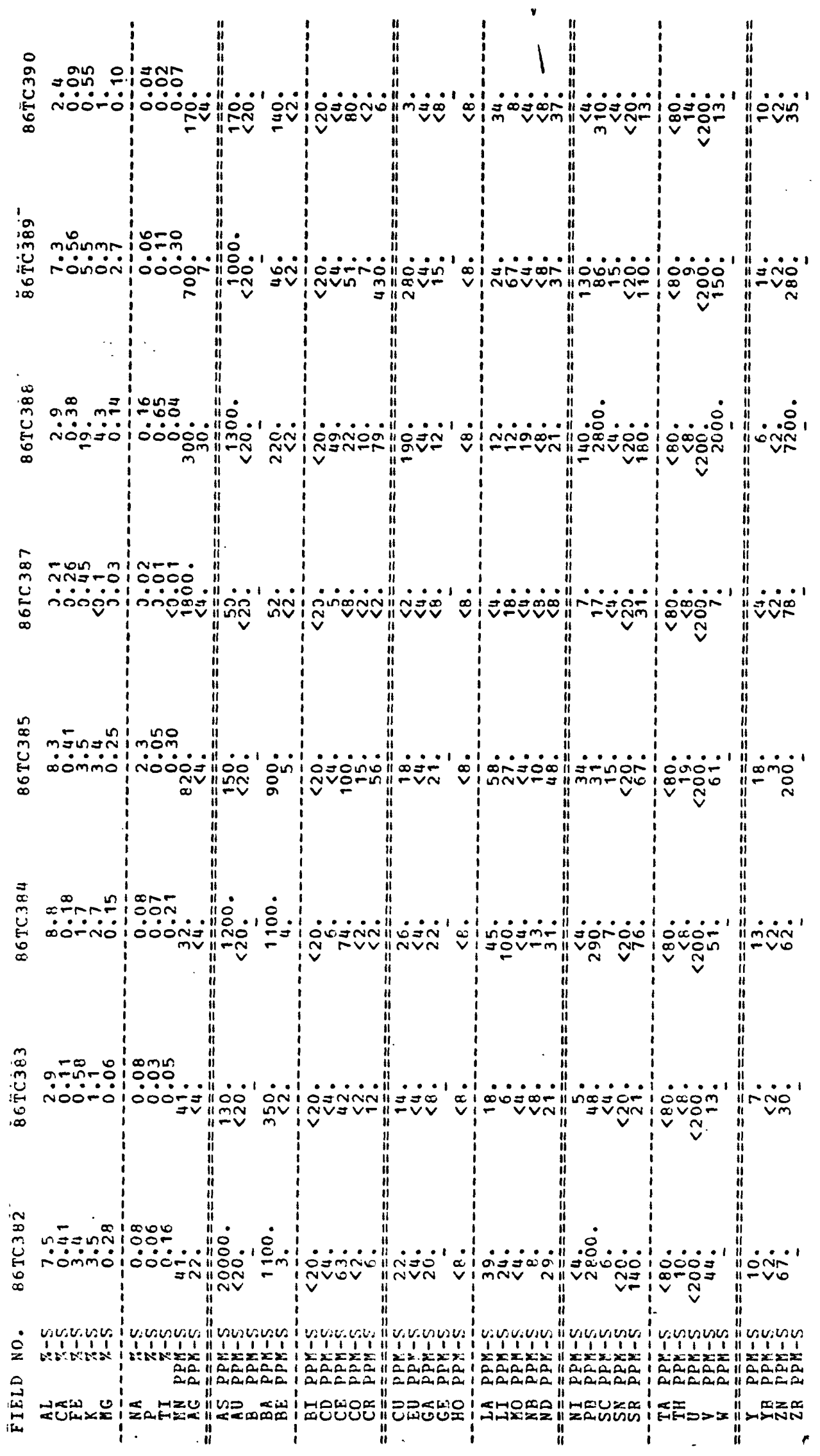




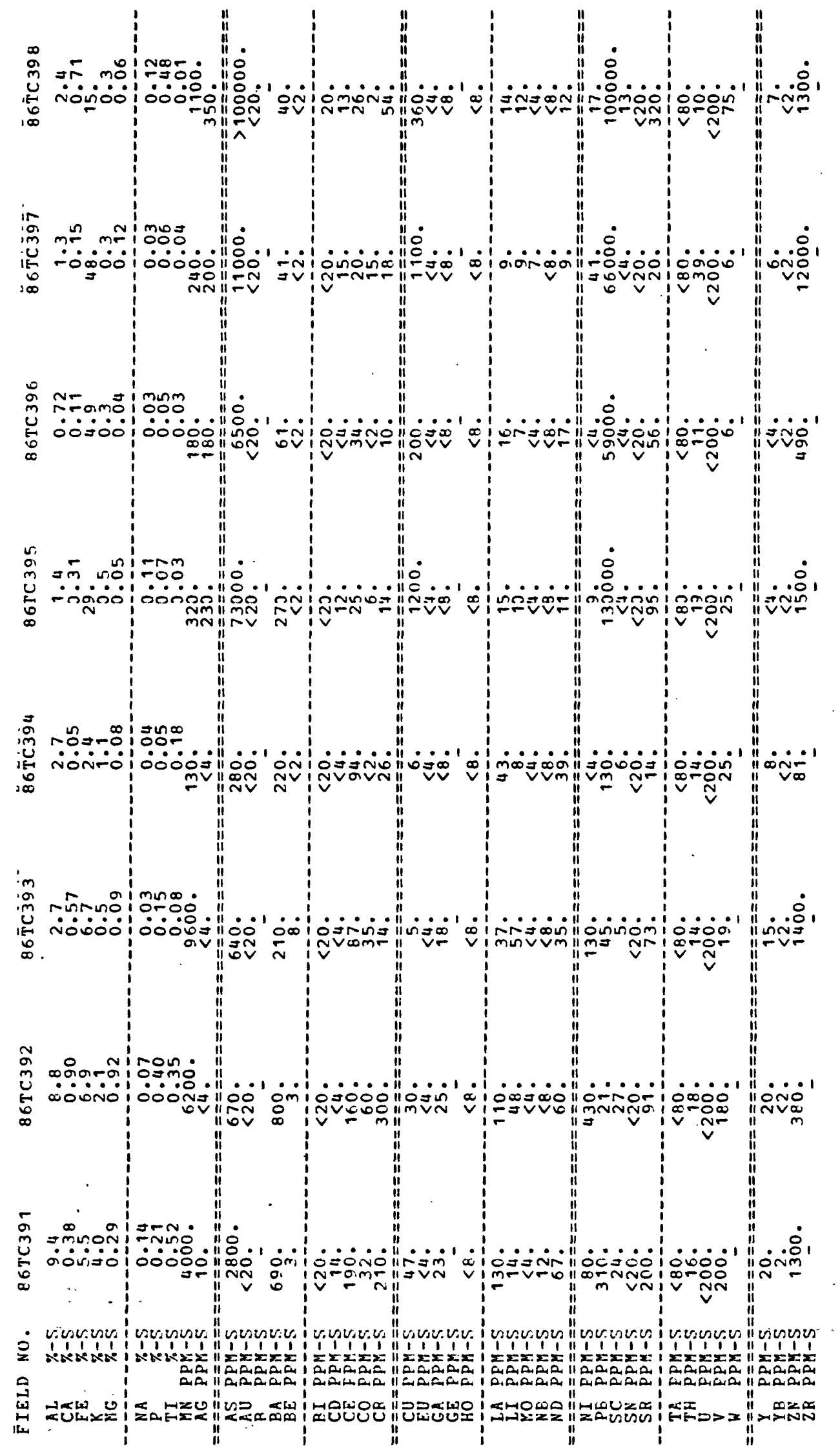




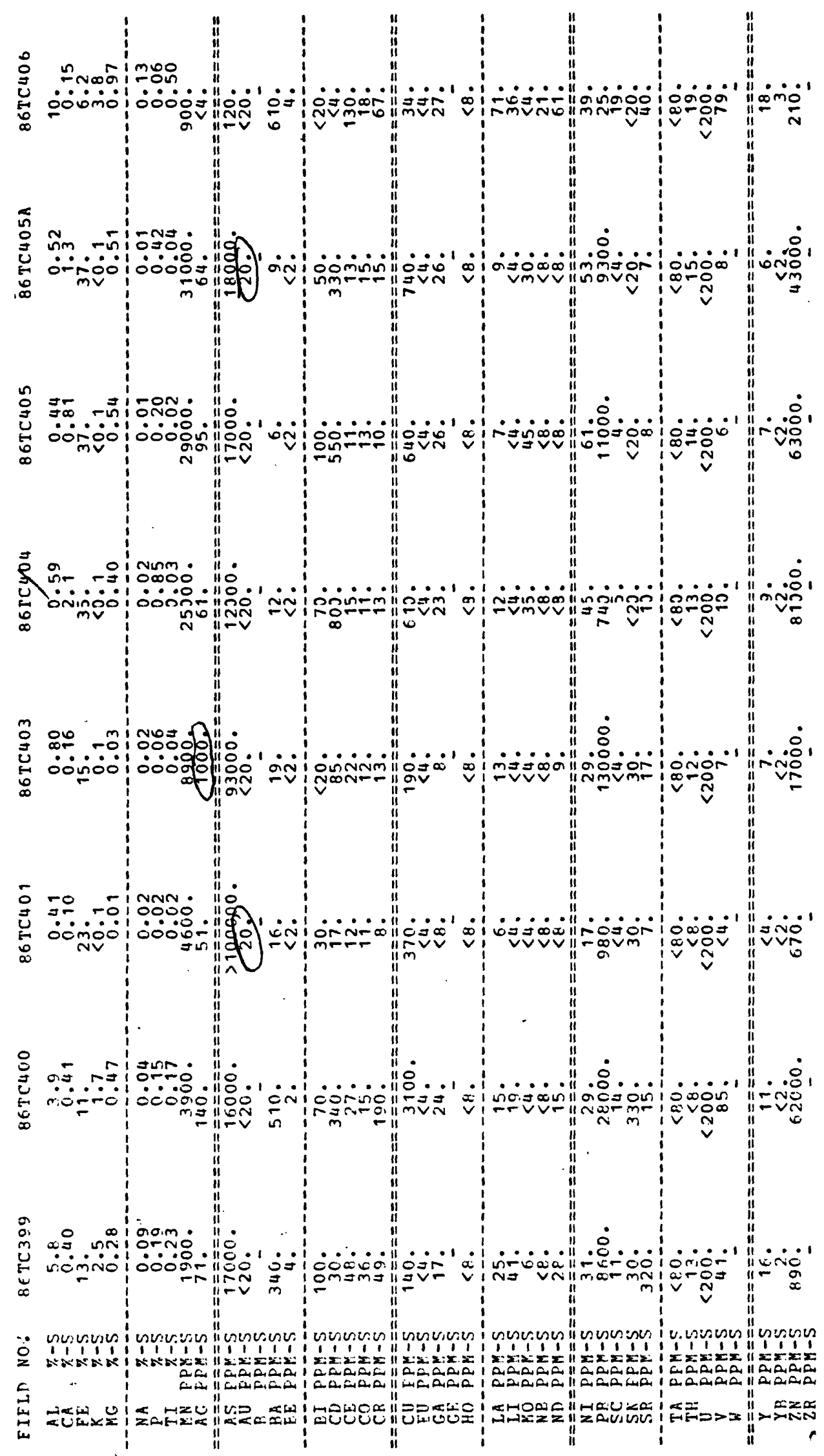




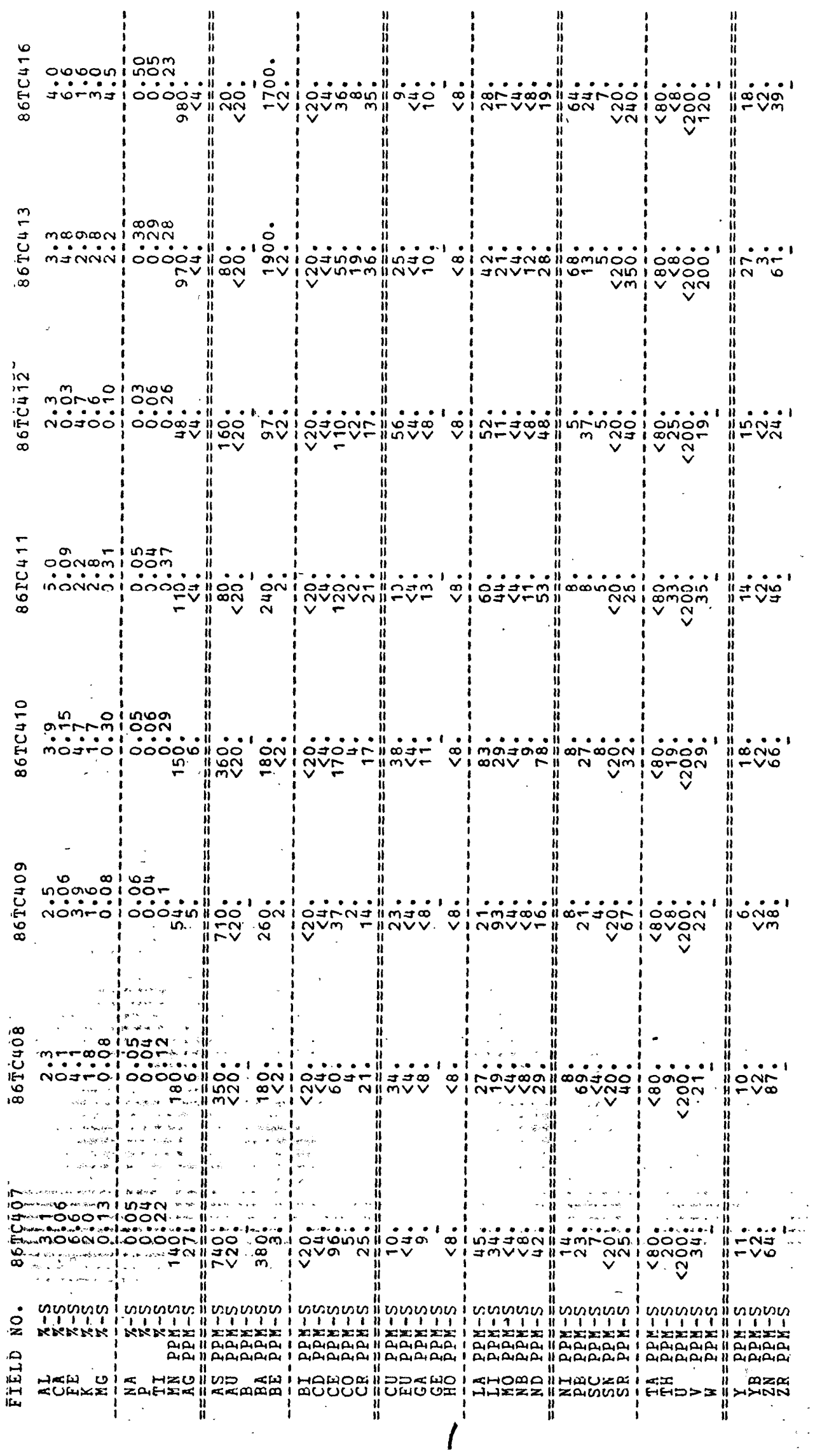




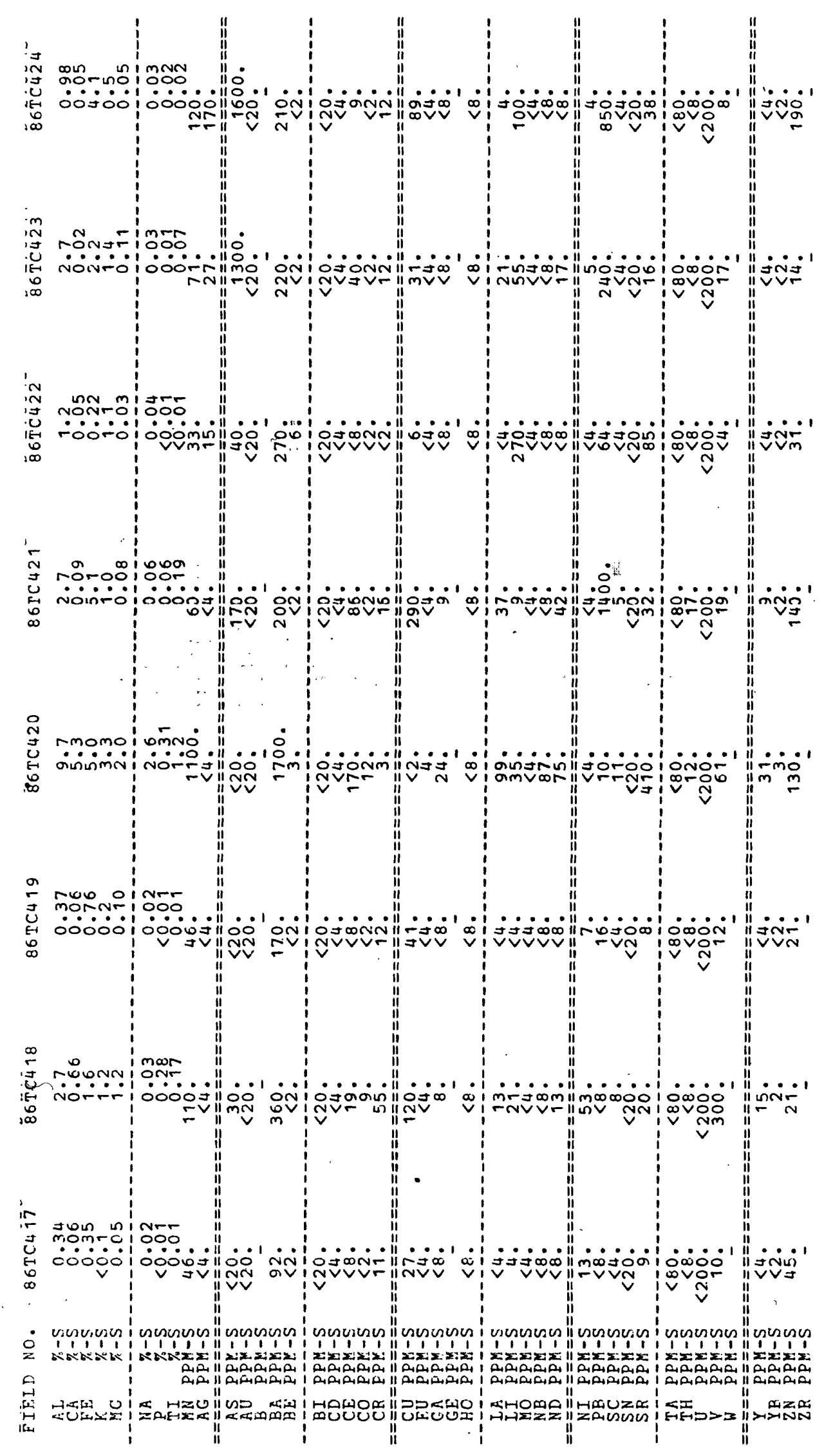




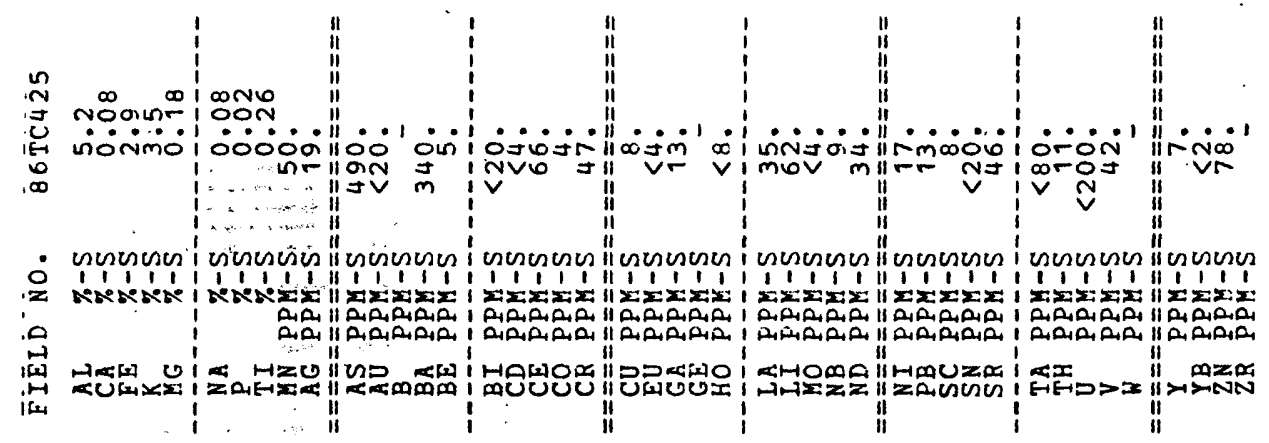



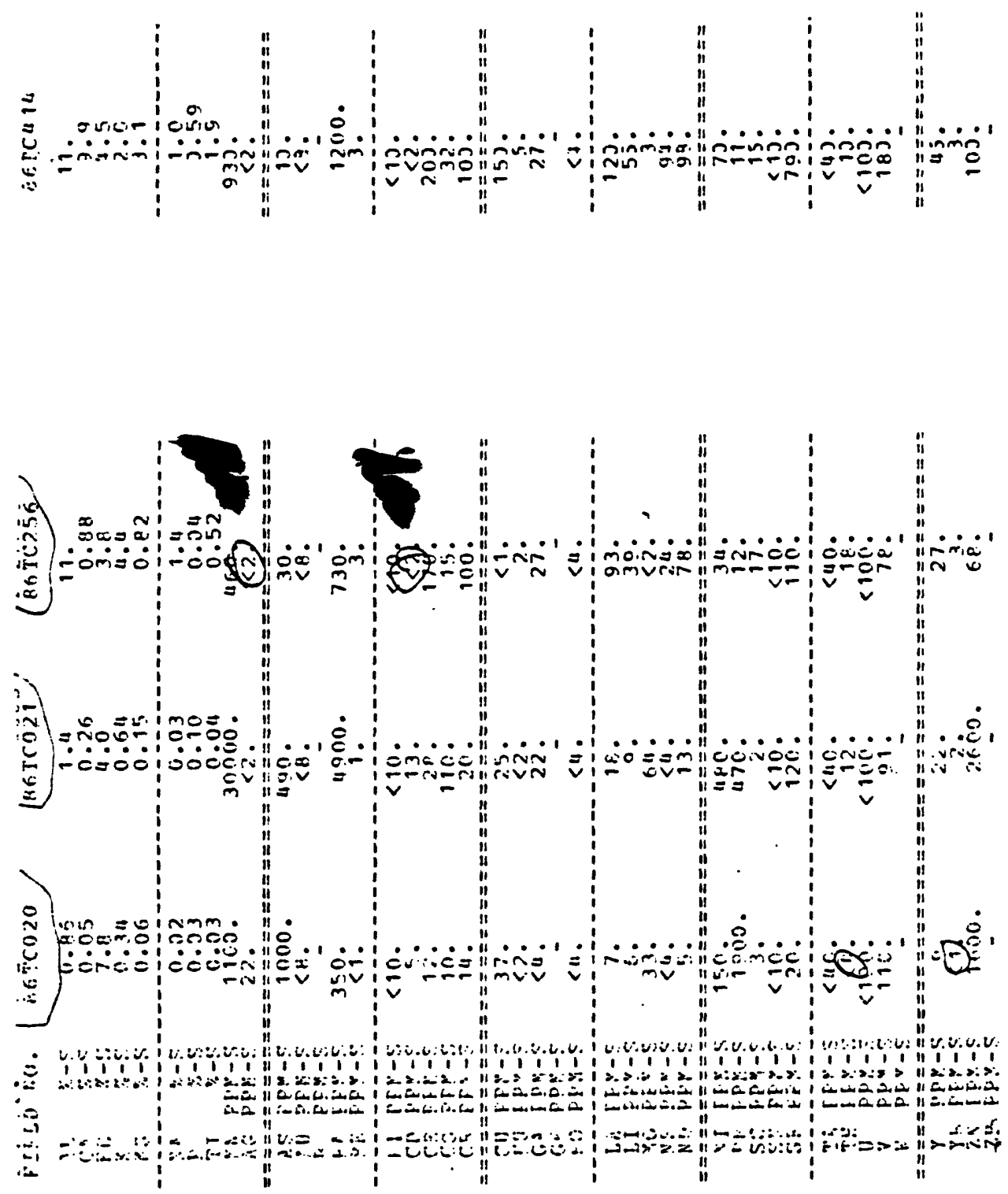


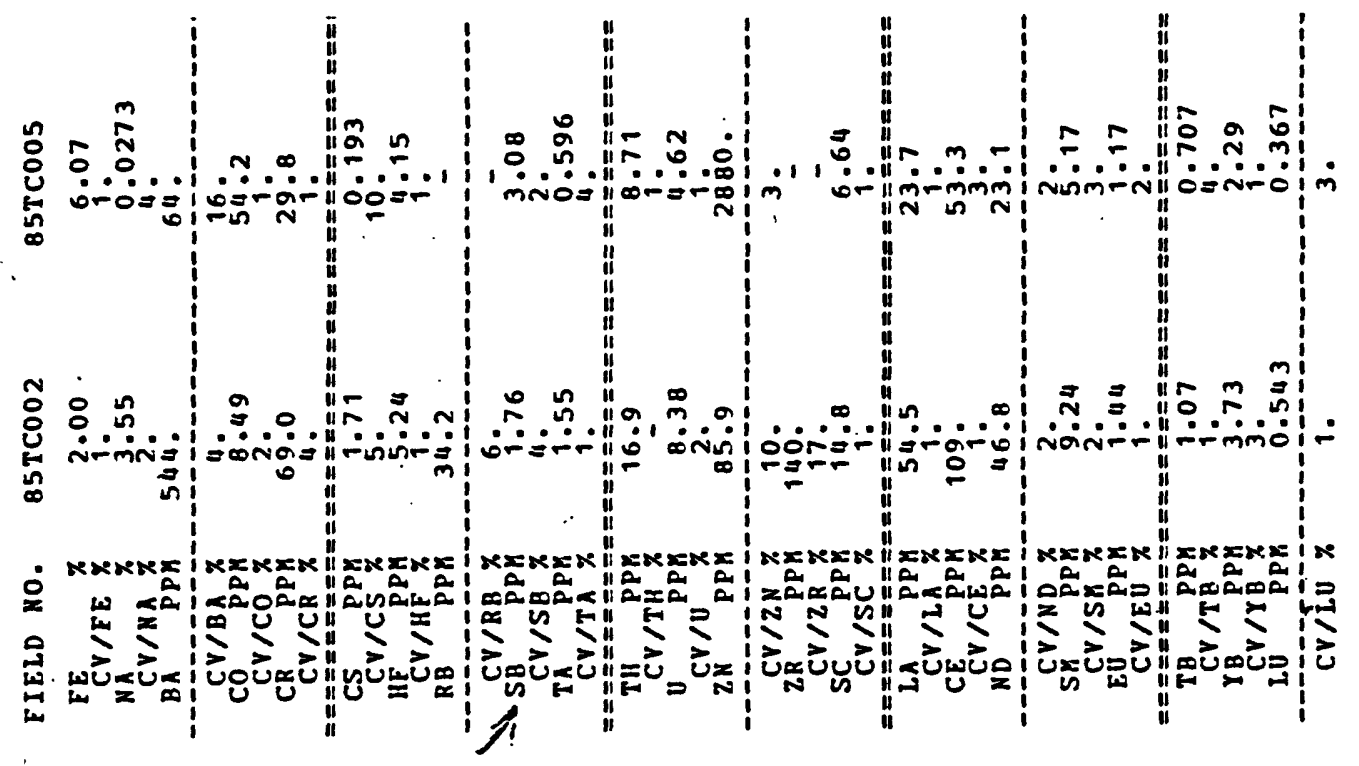




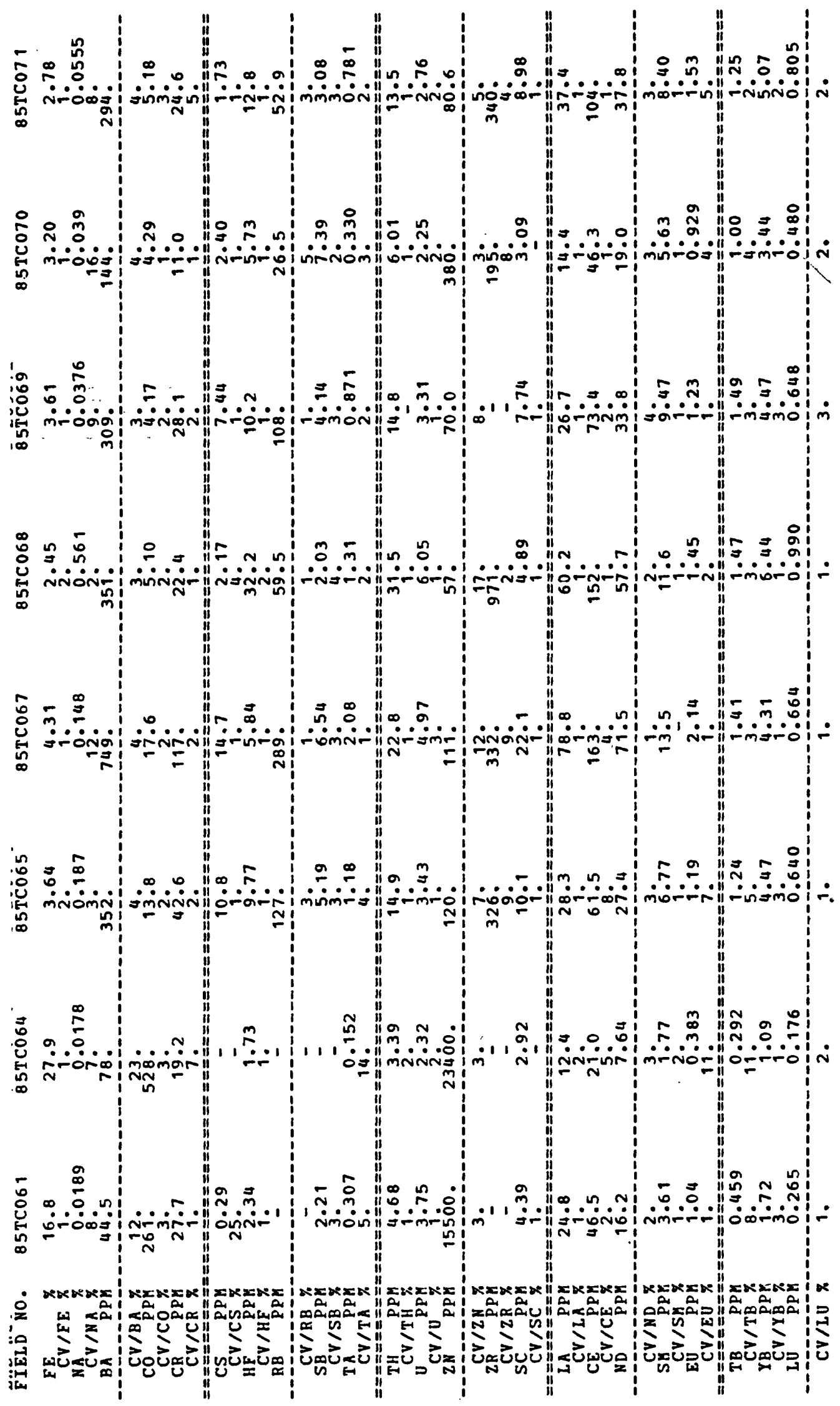




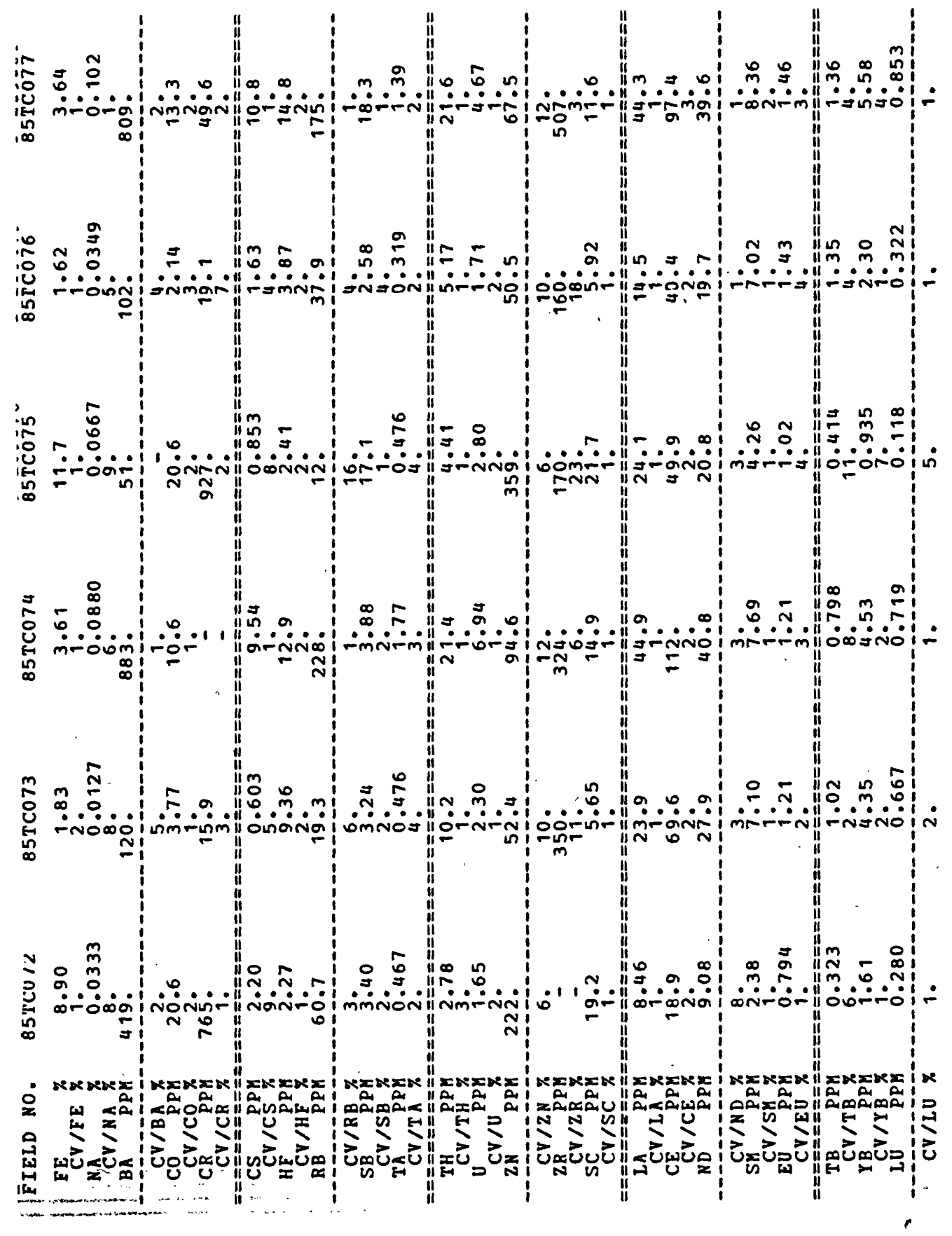




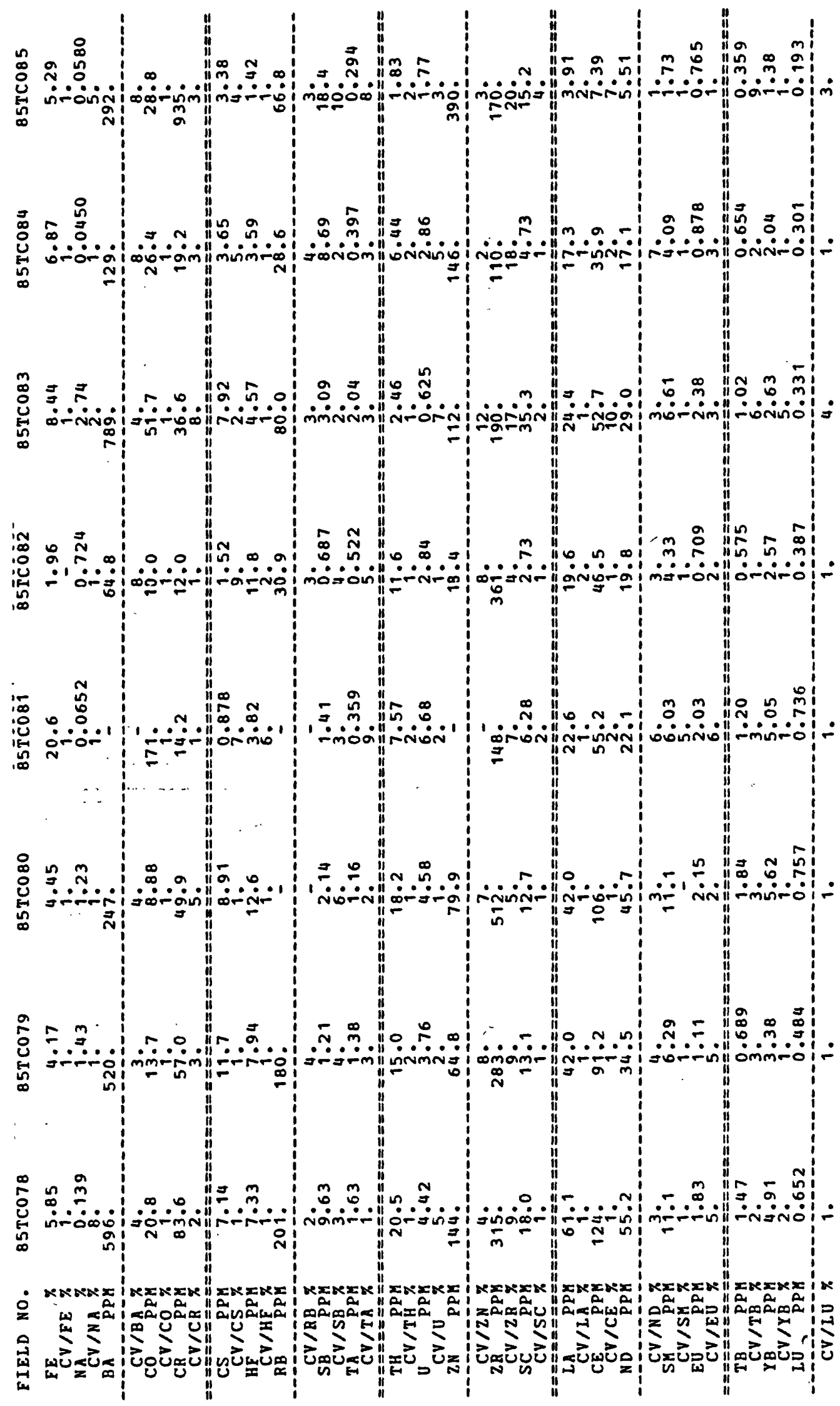




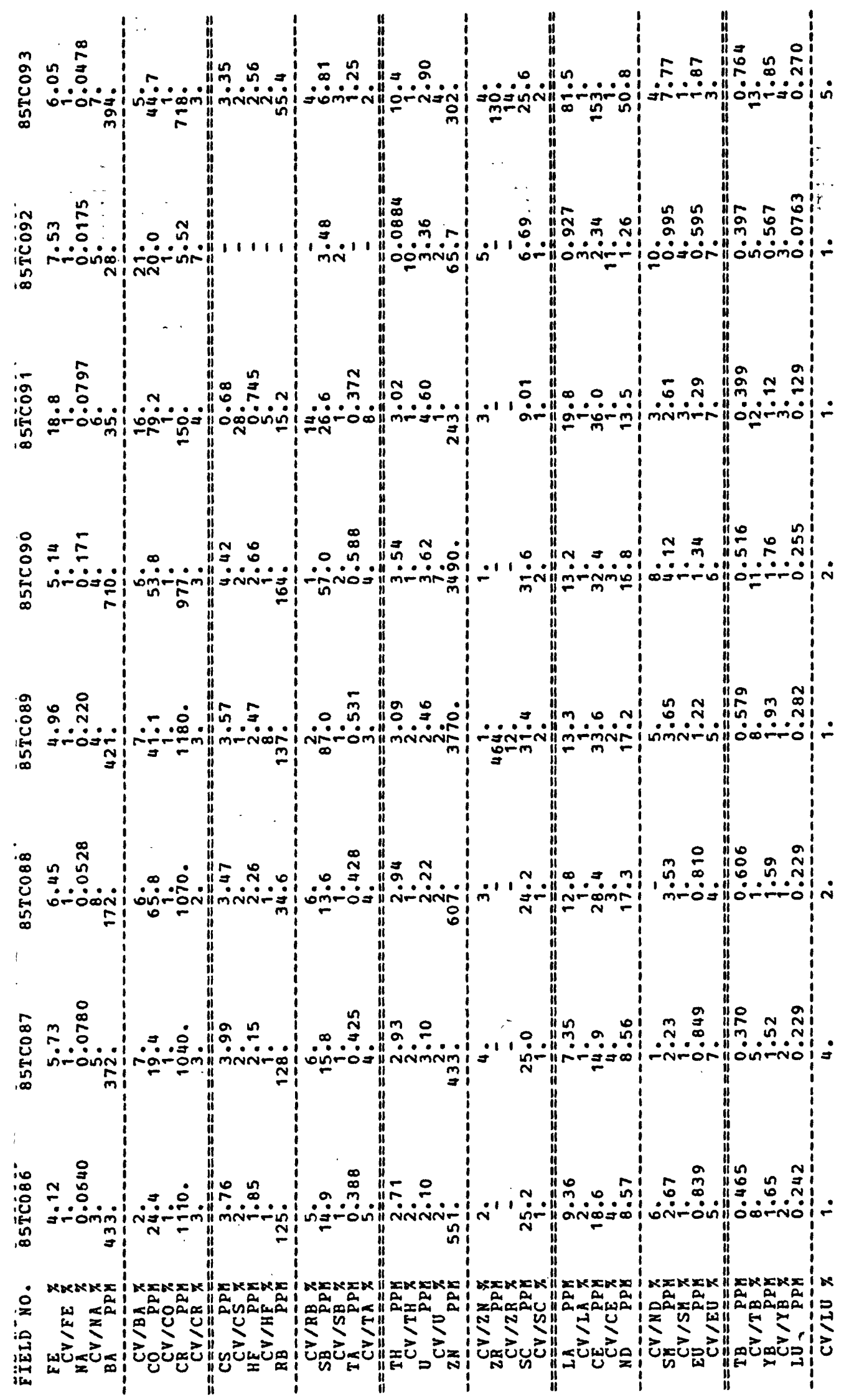



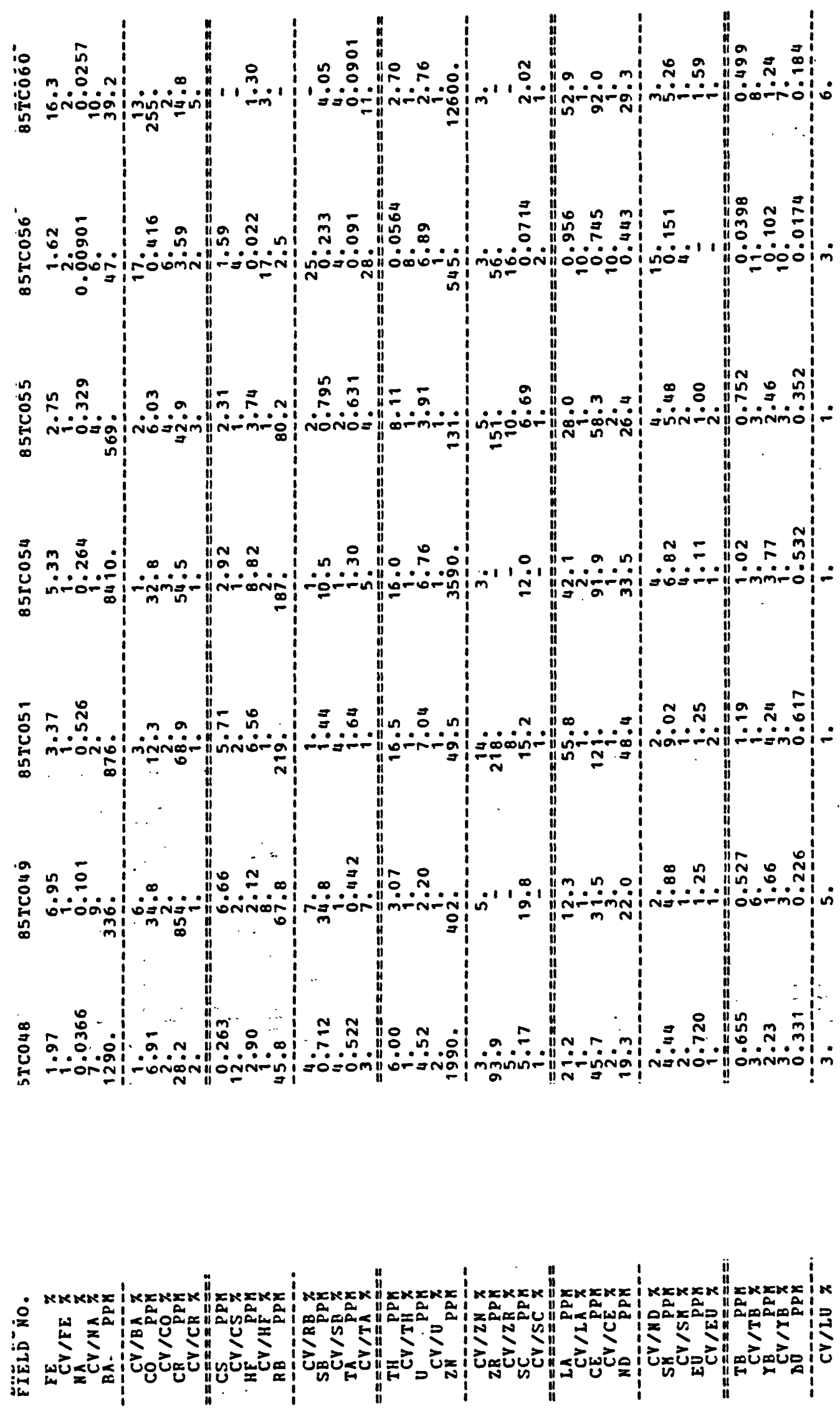


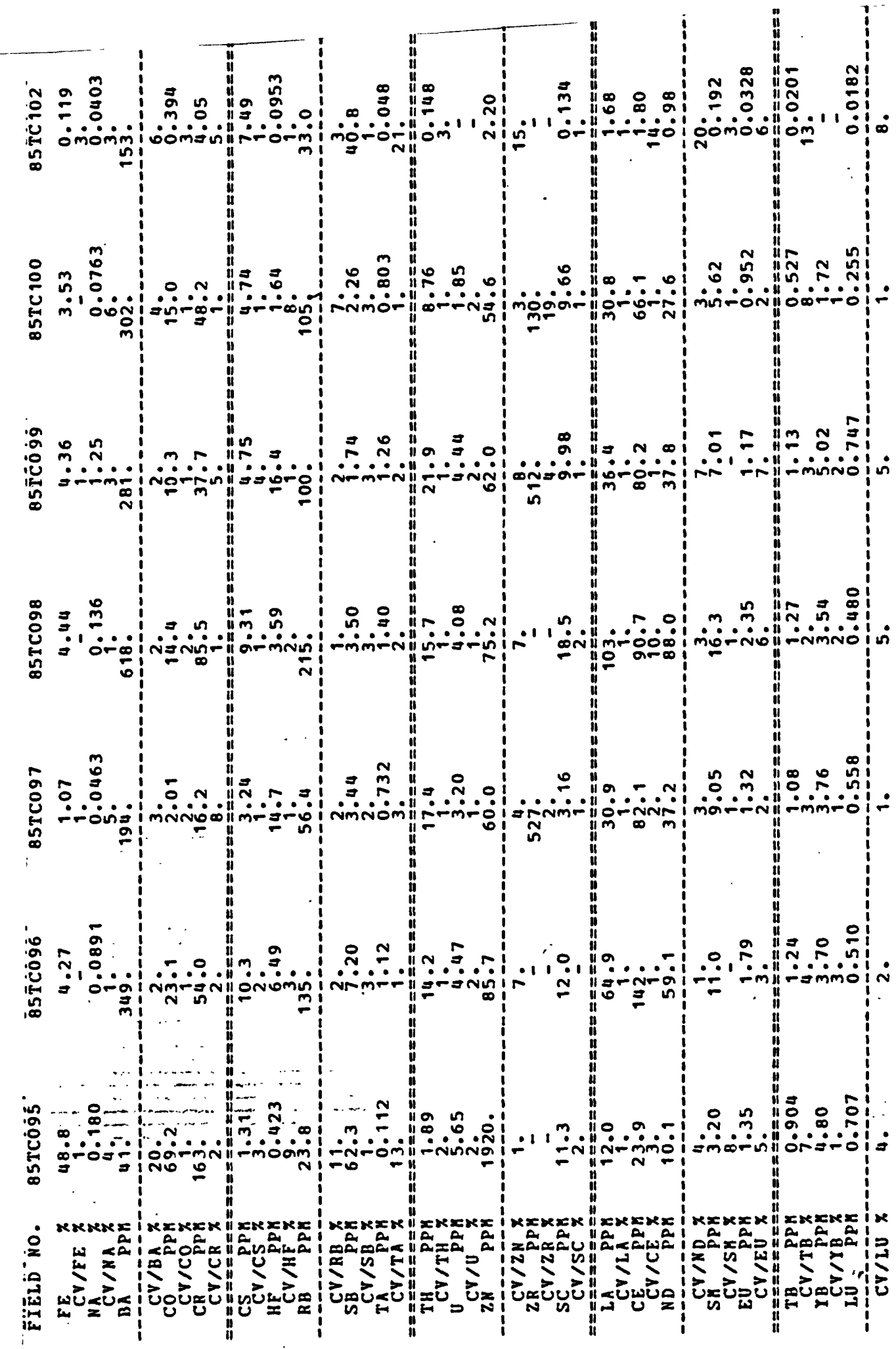



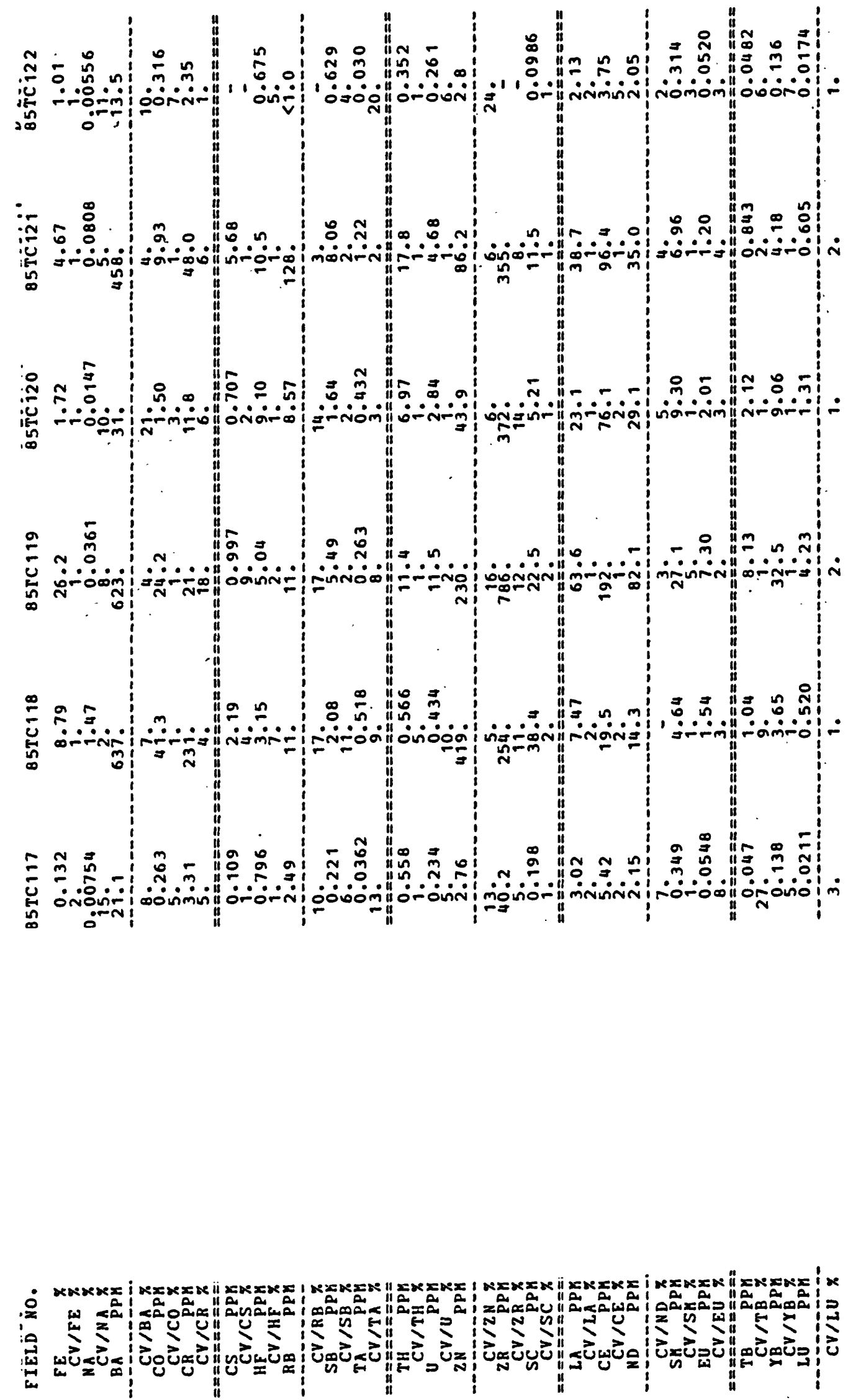


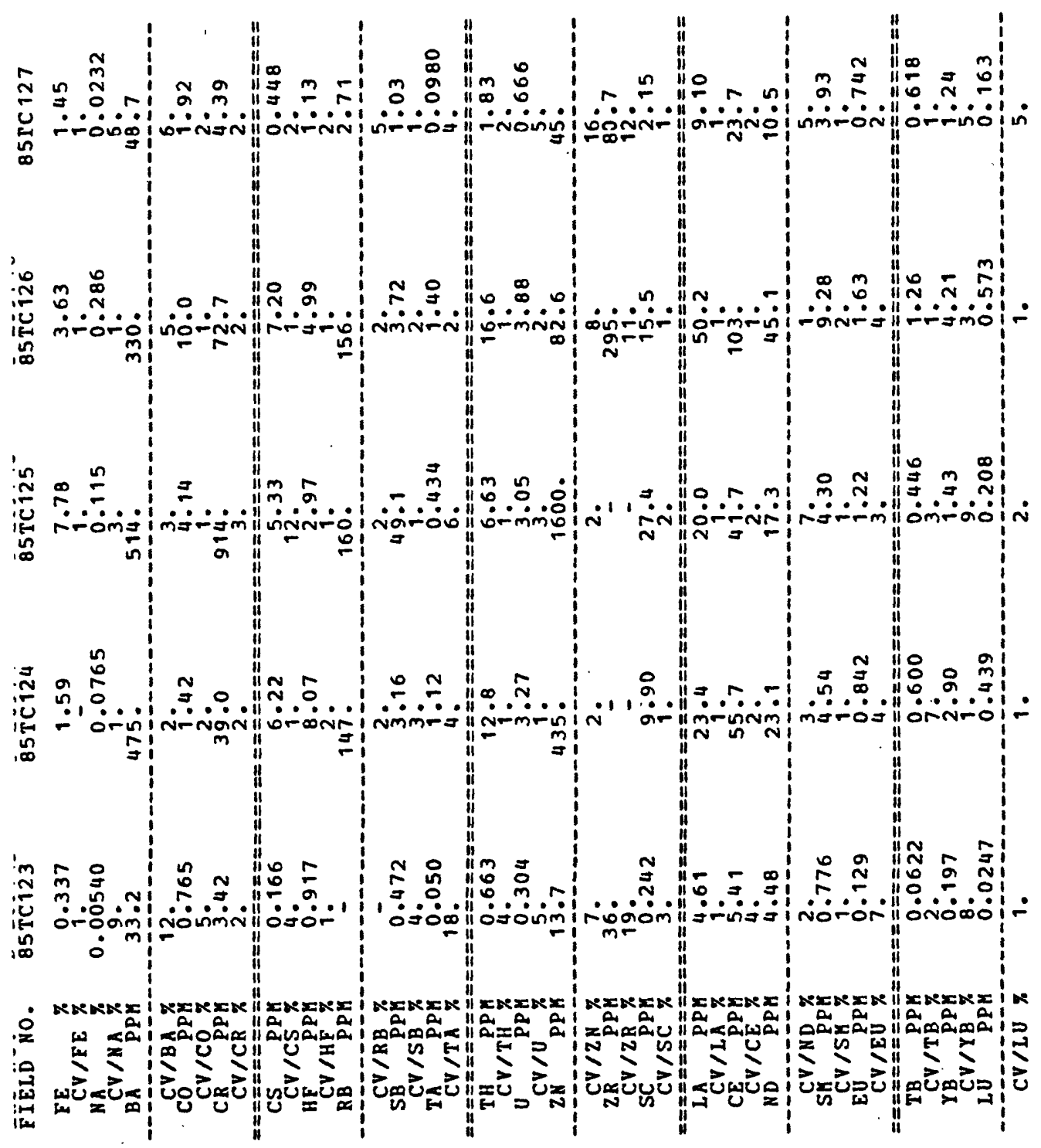




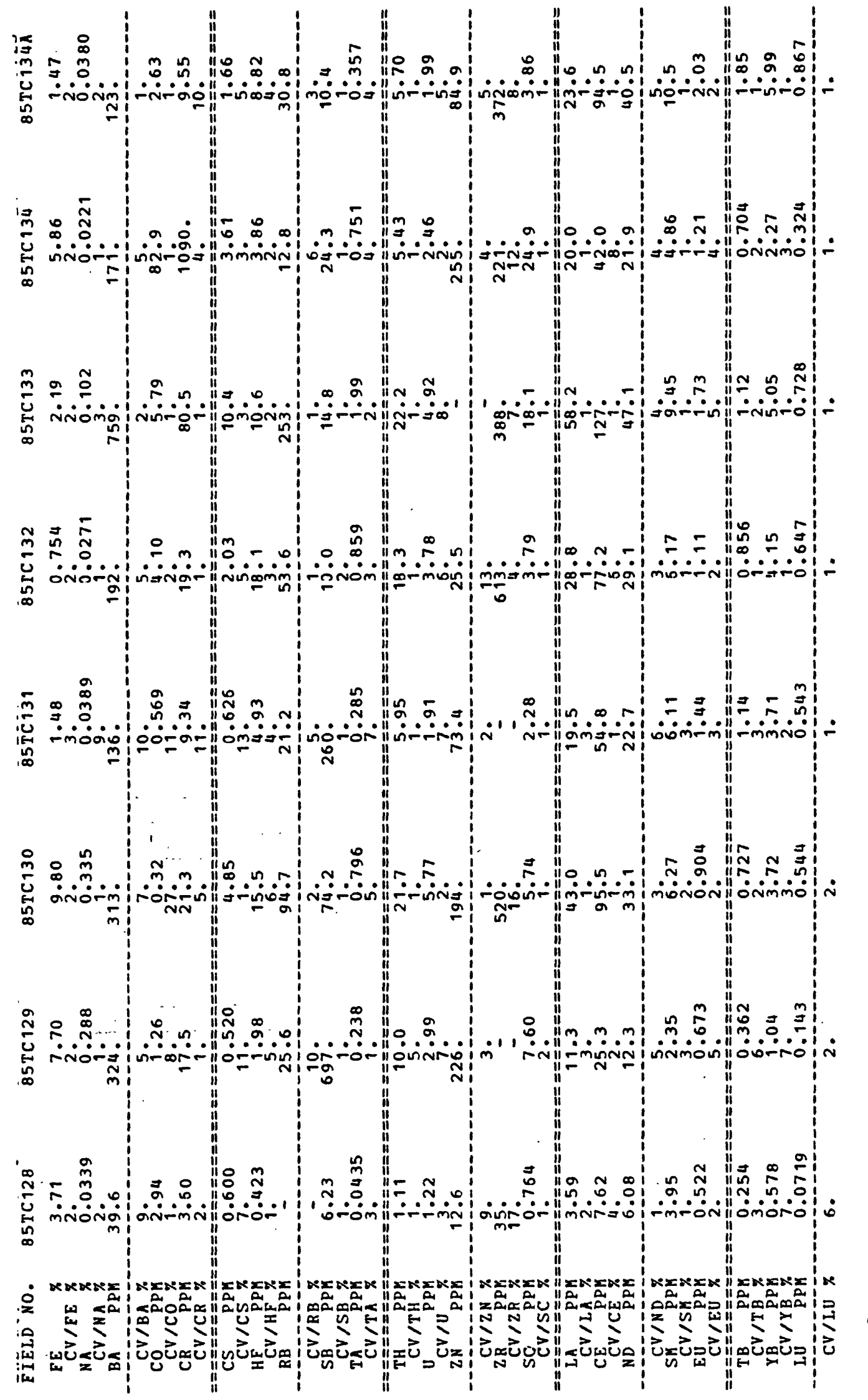




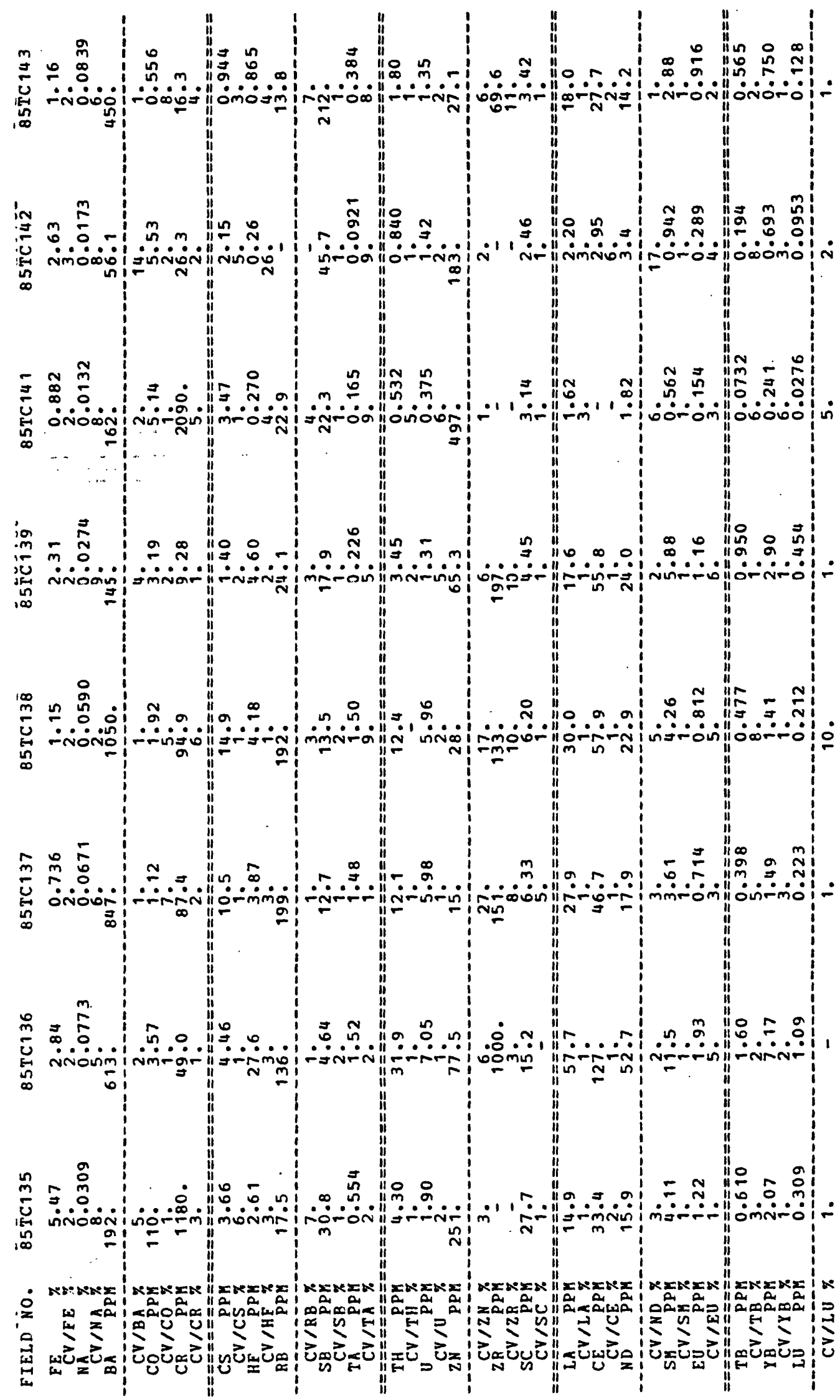




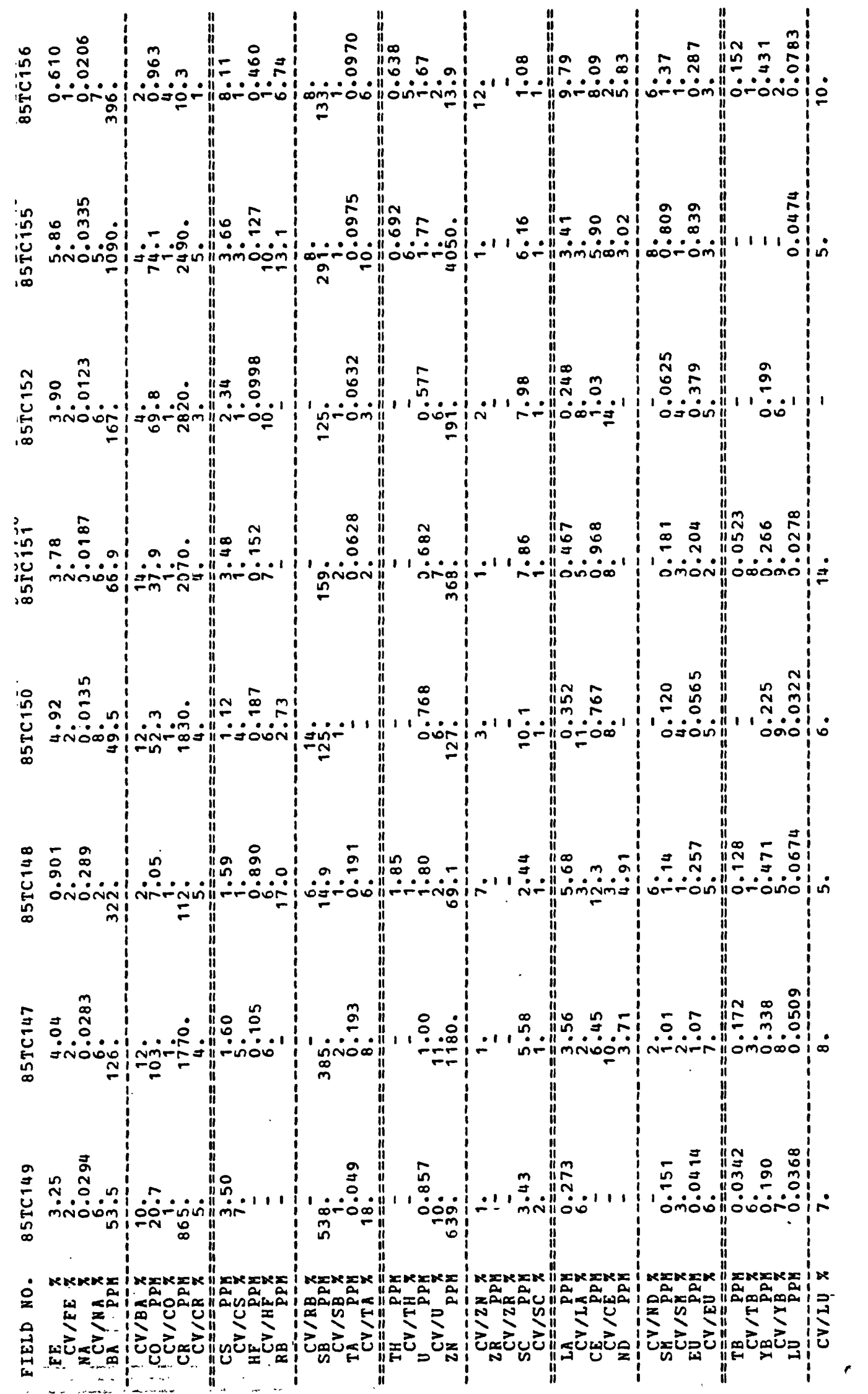




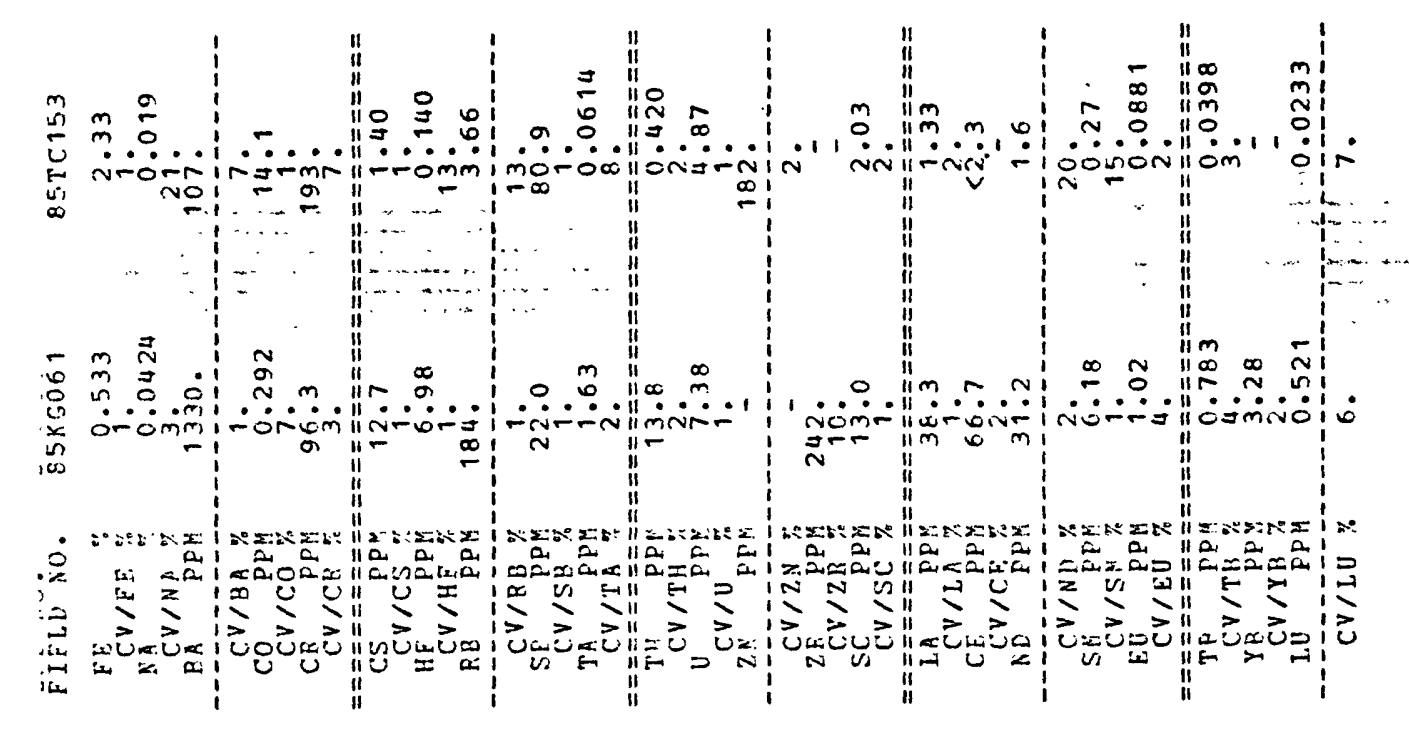

99 


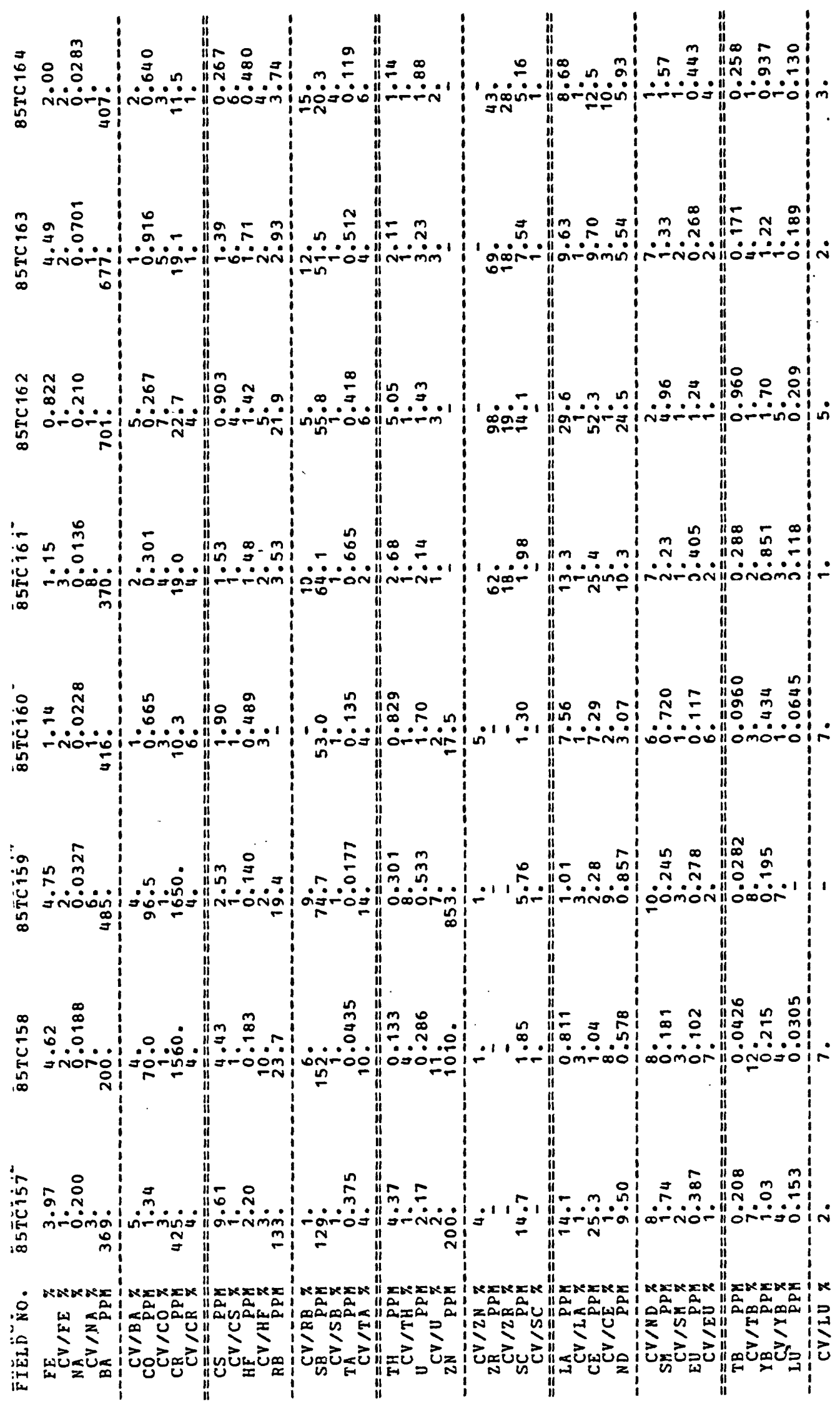




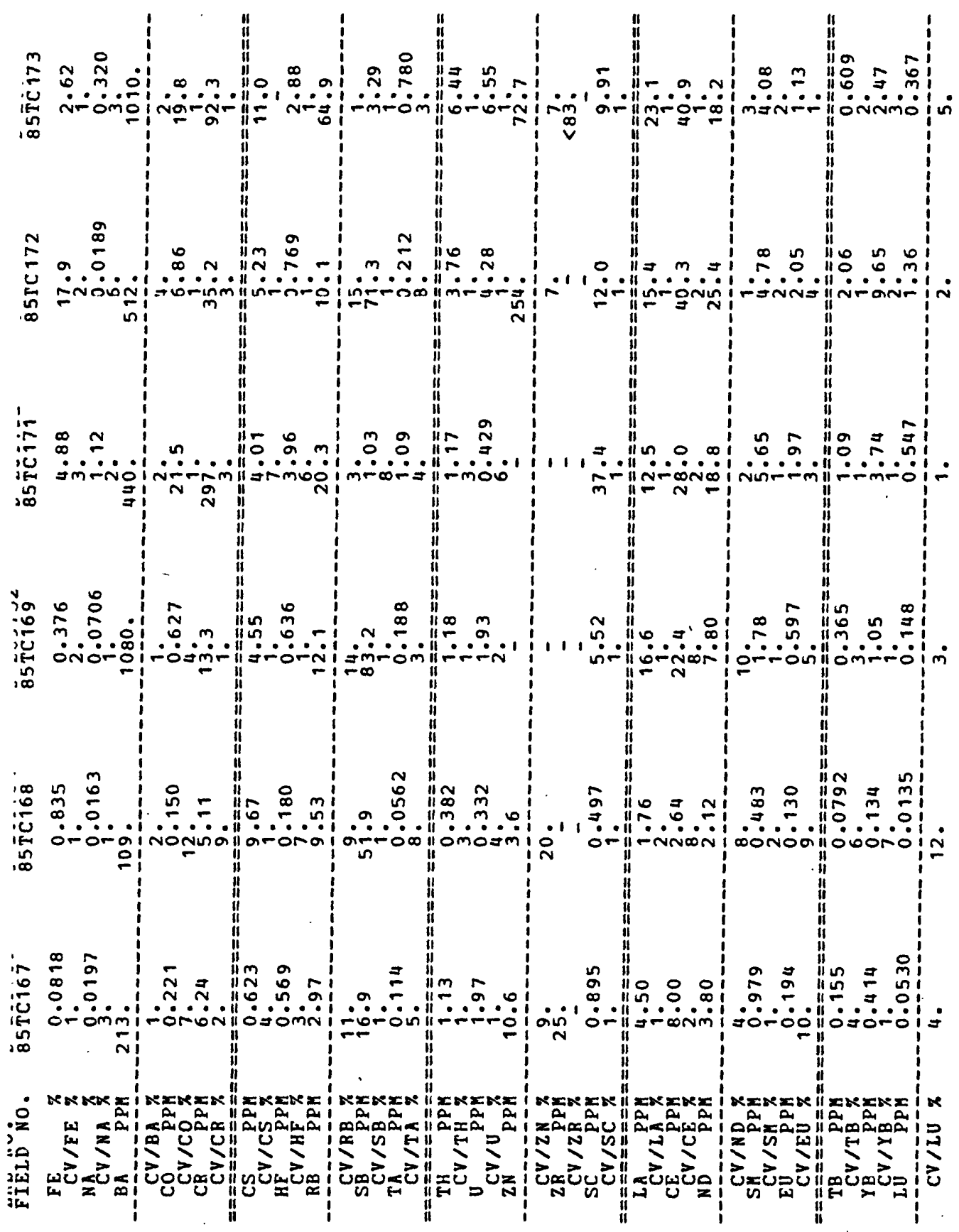




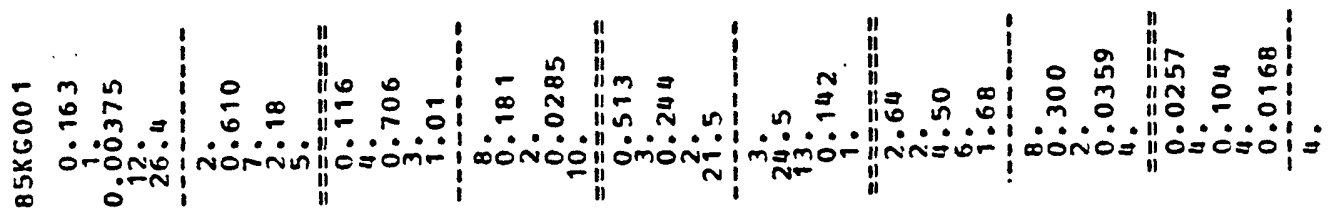

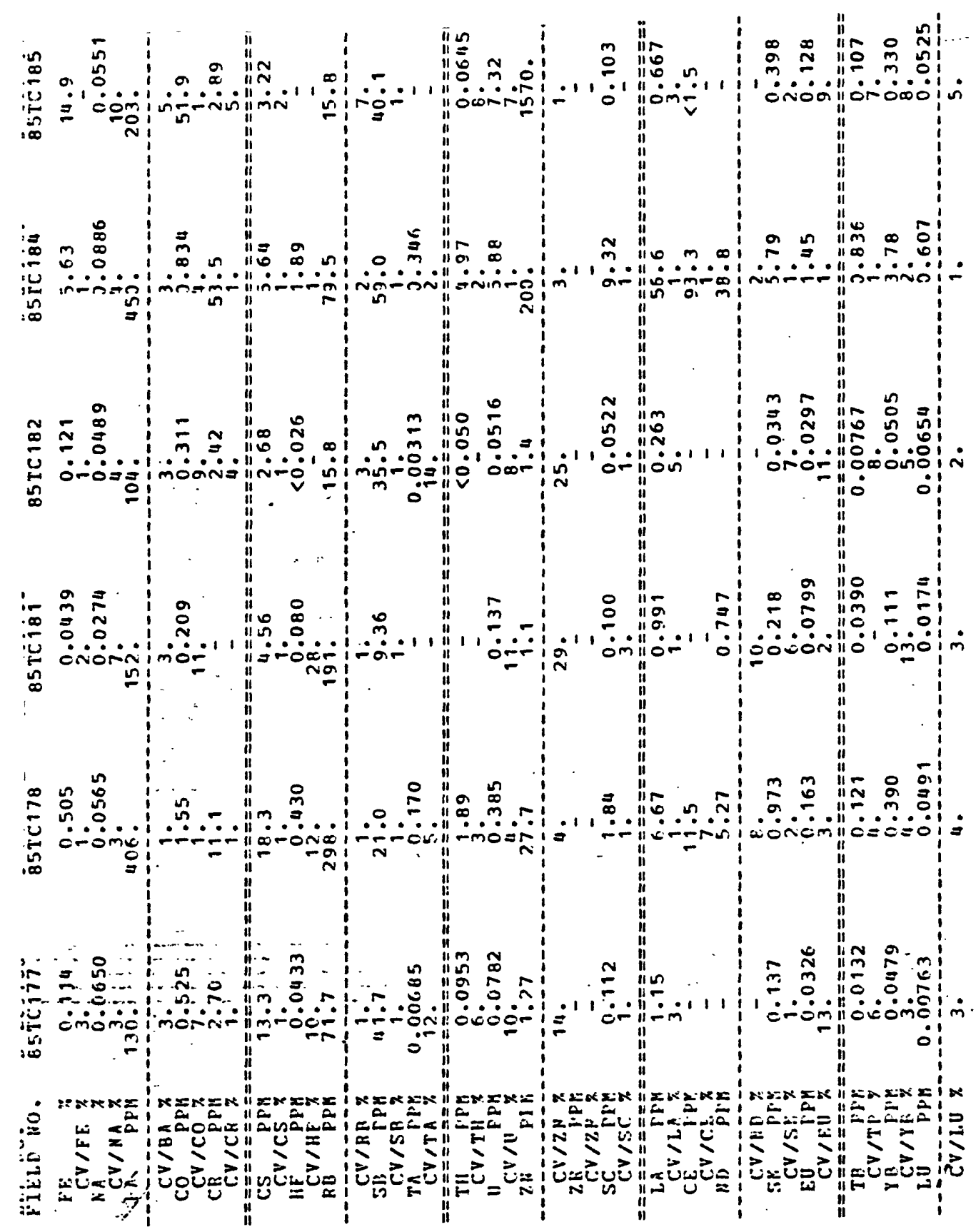




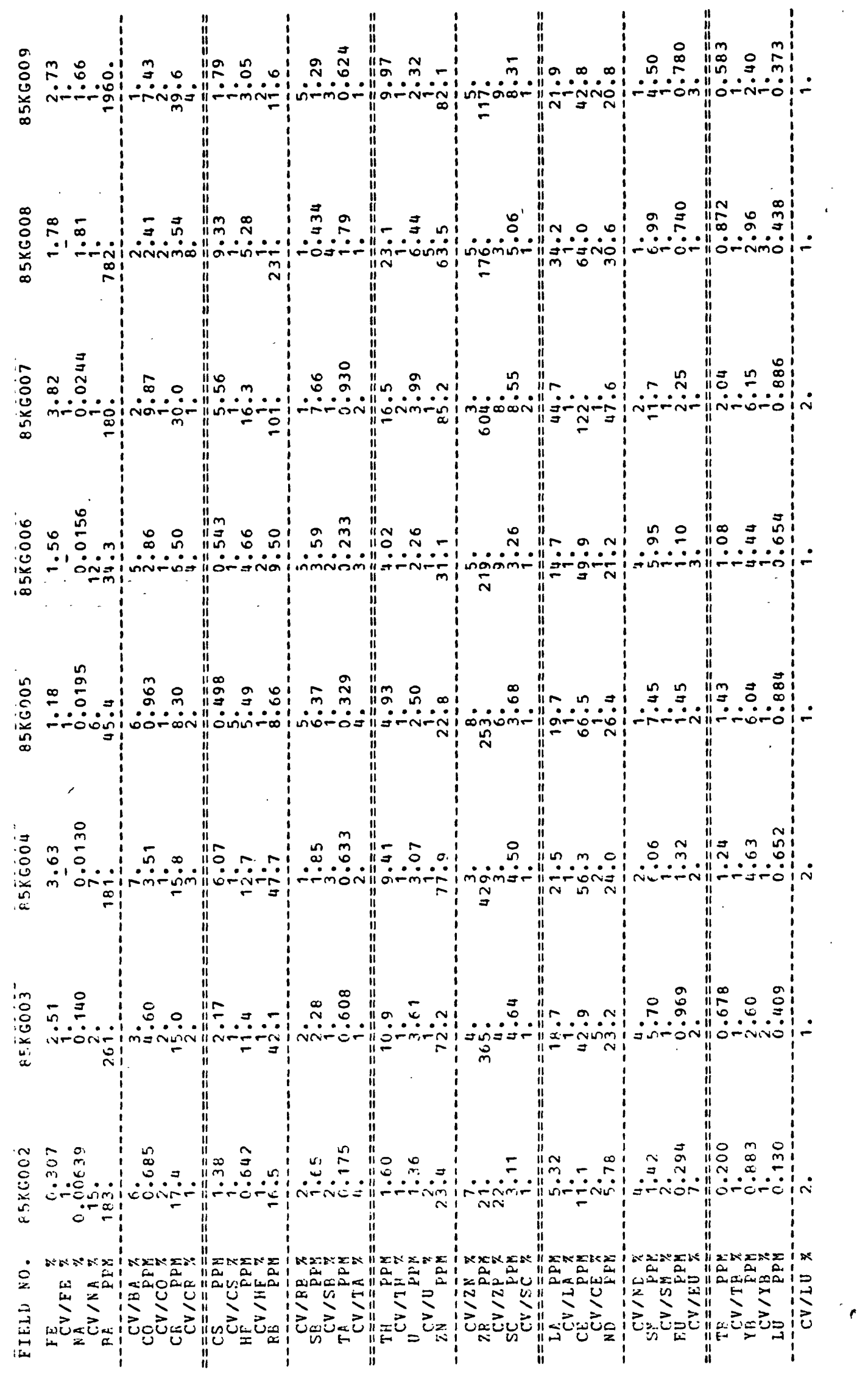




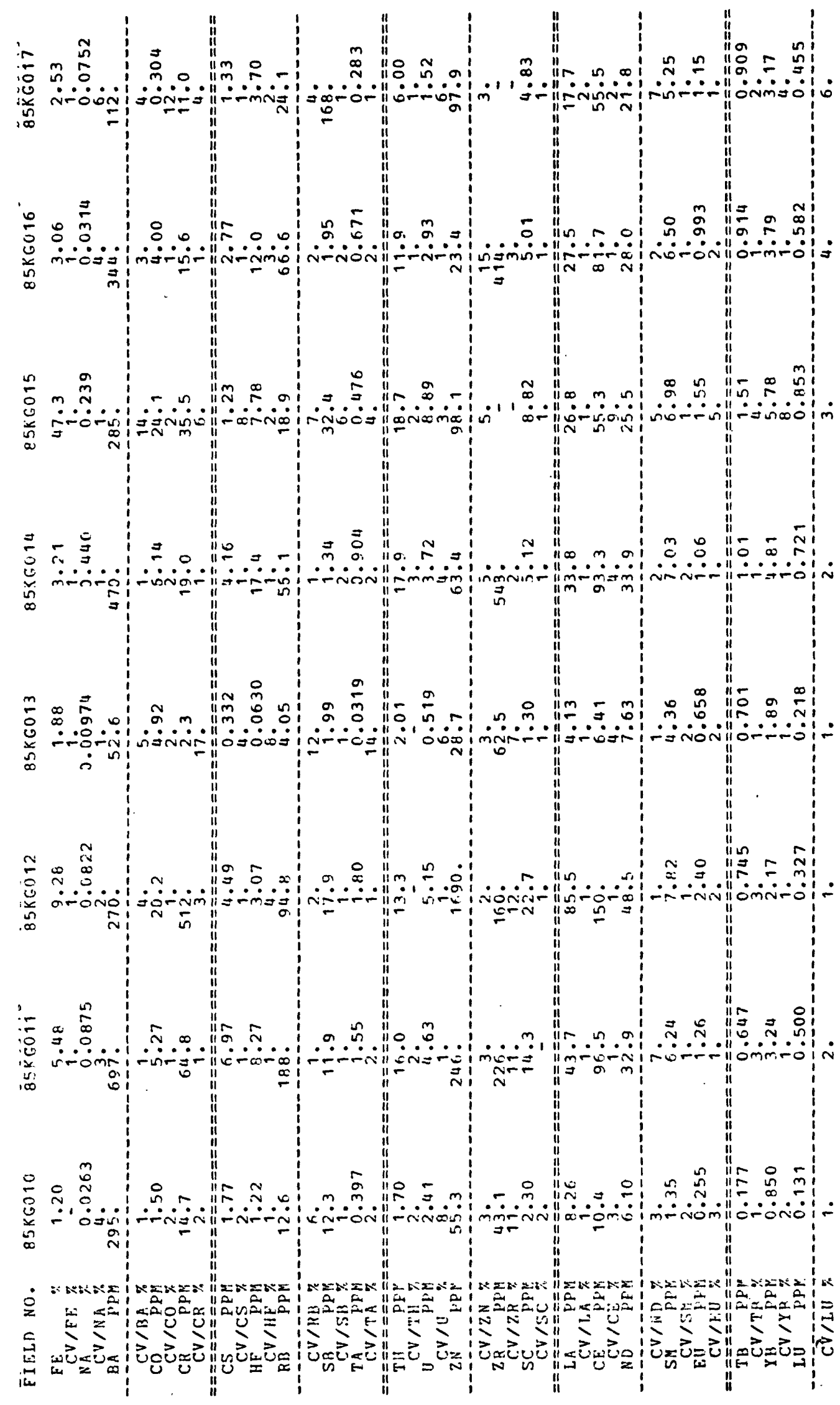




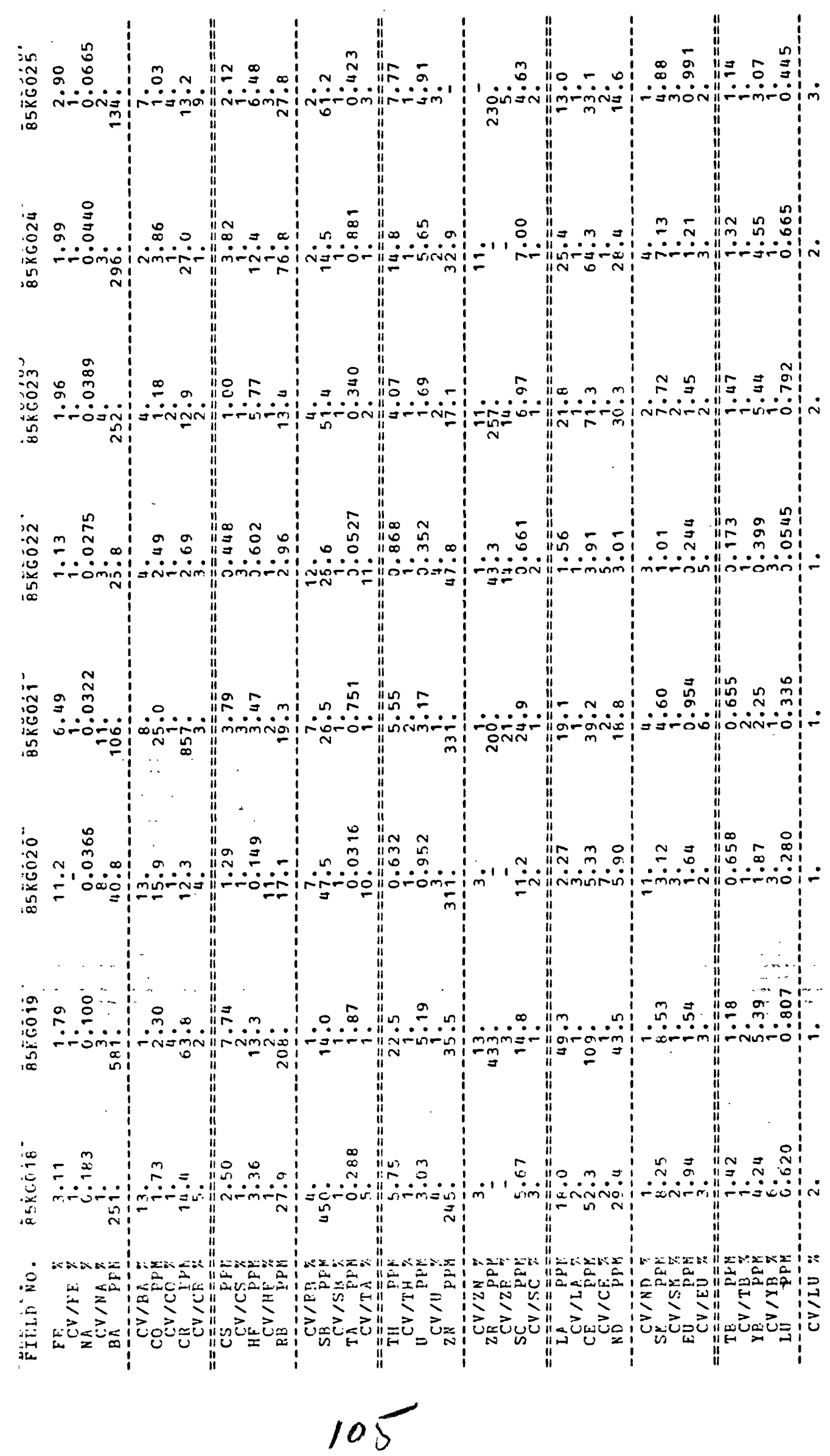




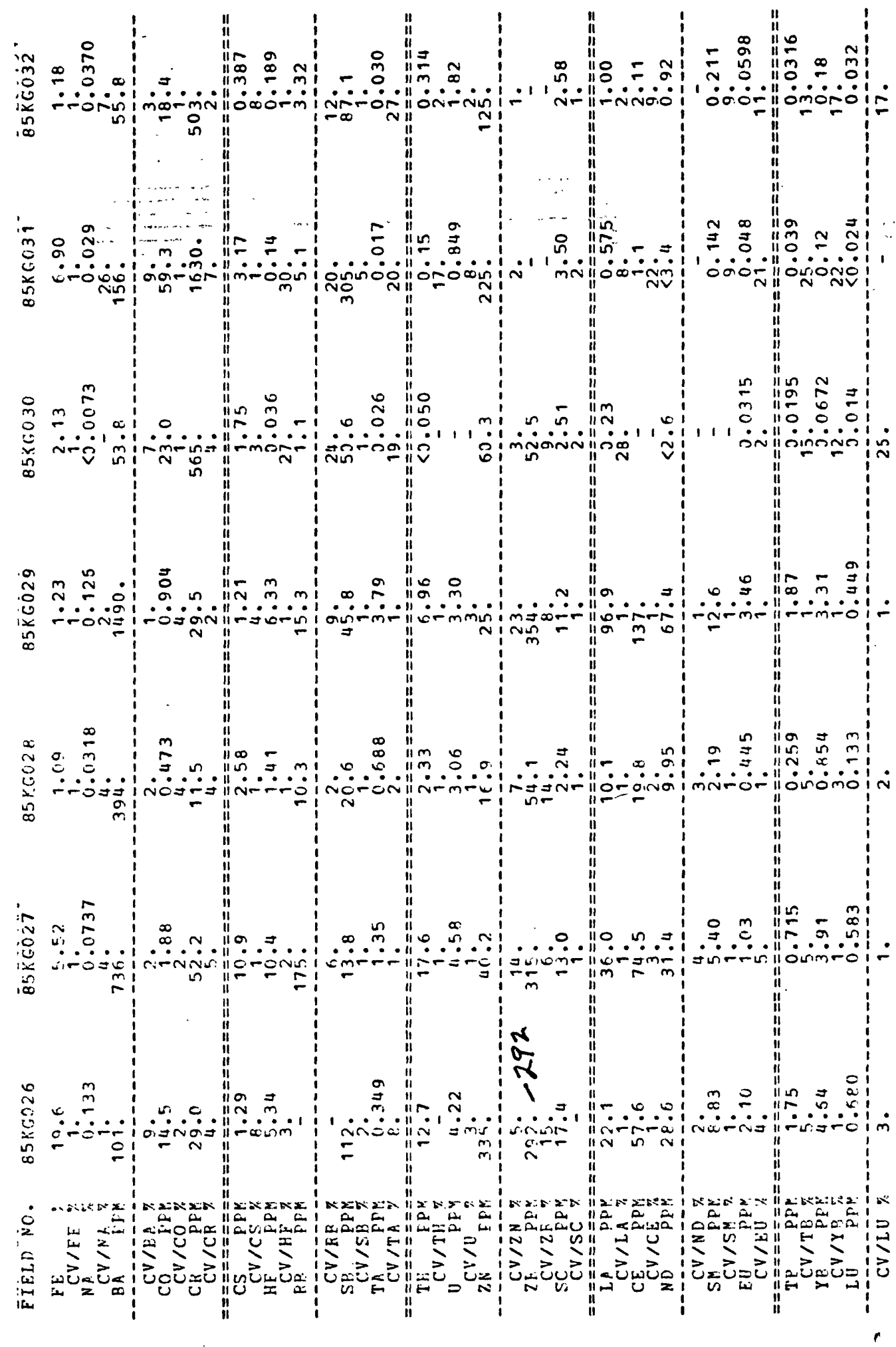




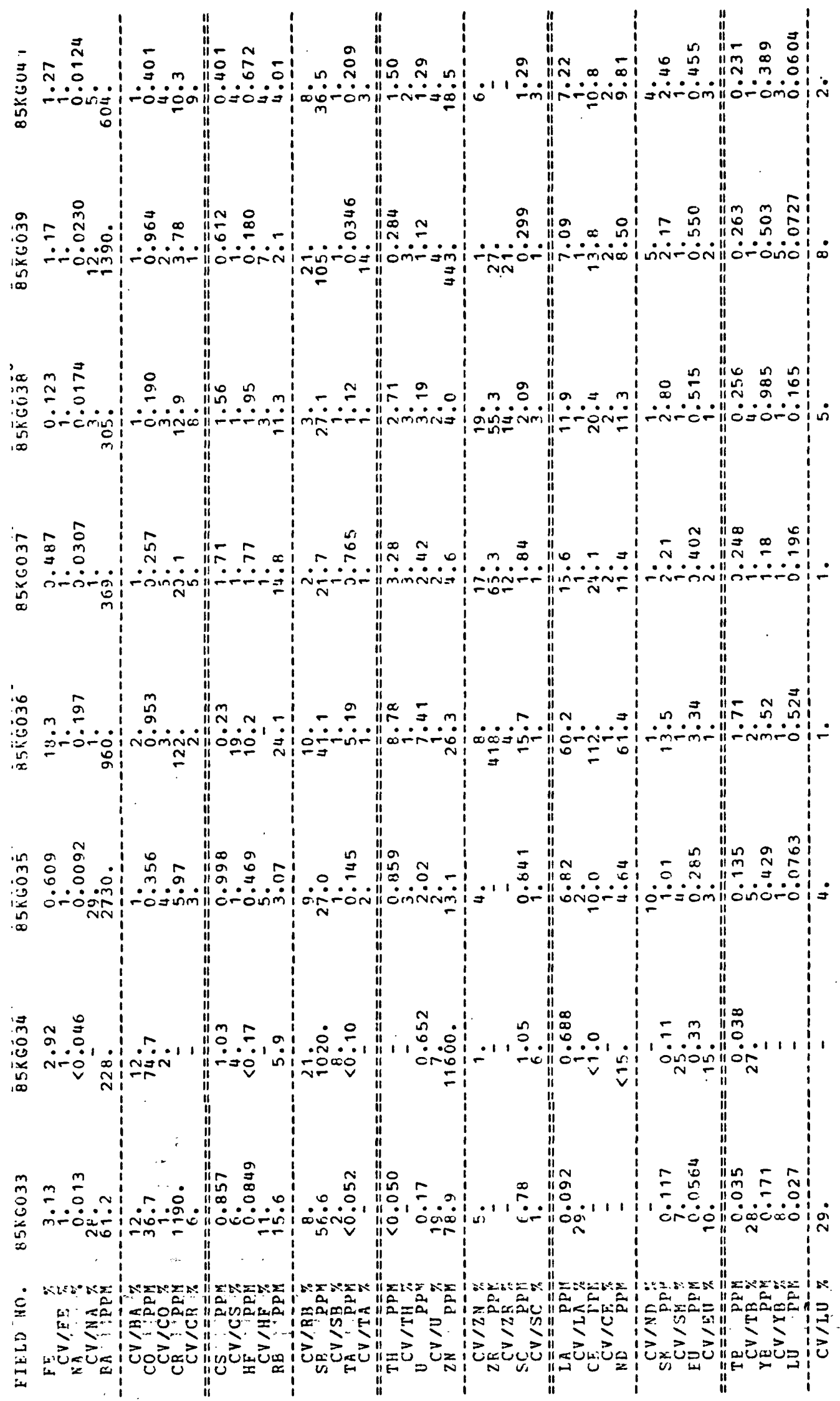




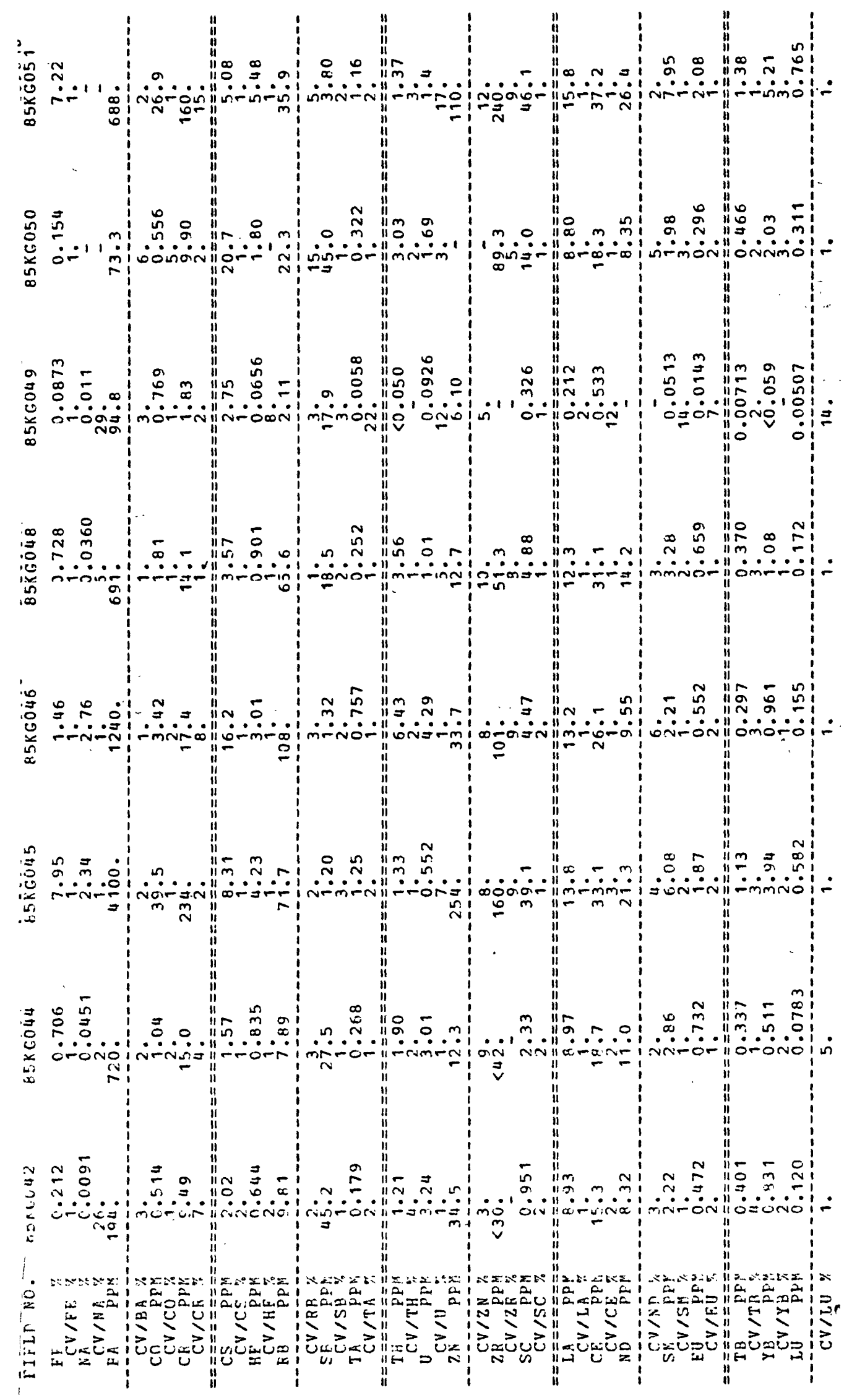




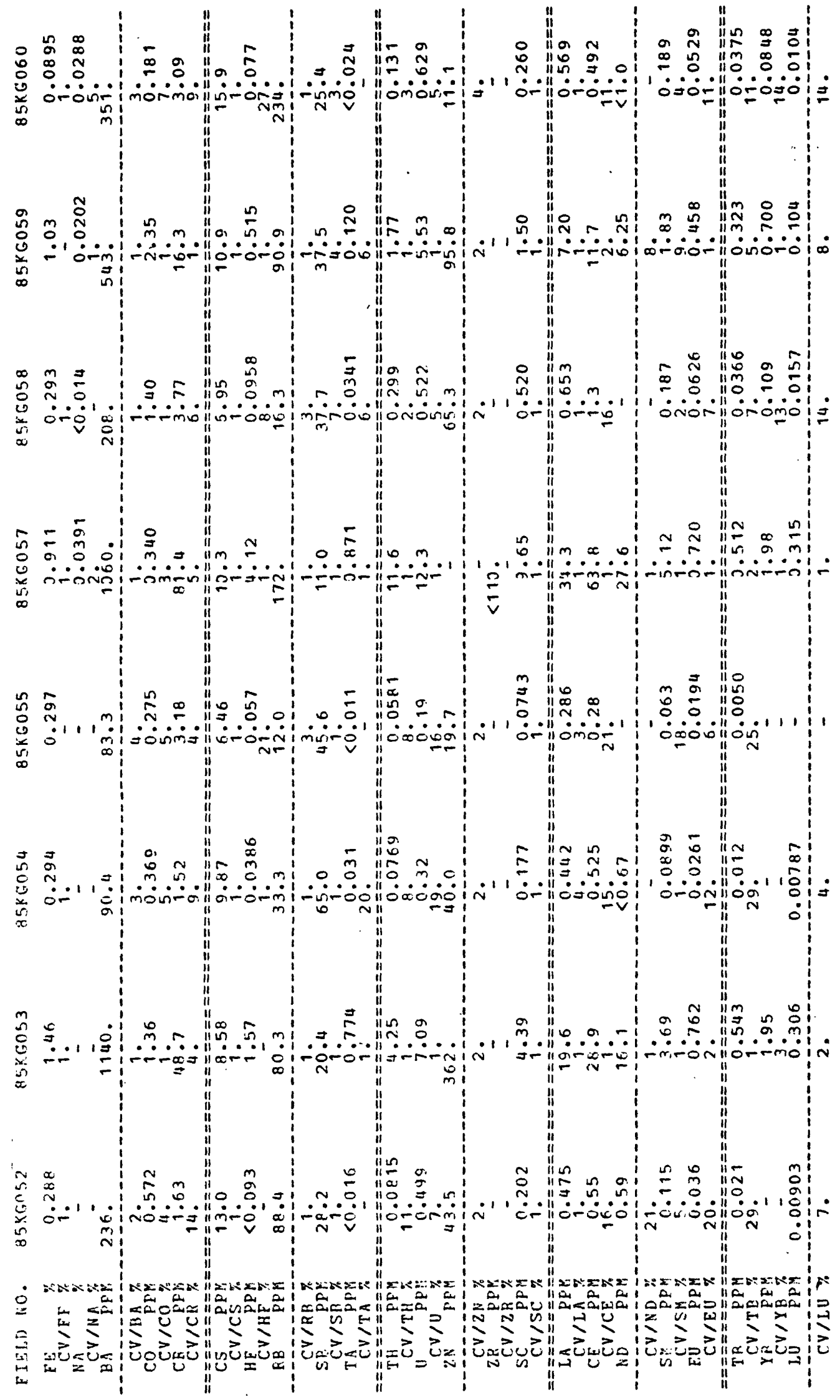




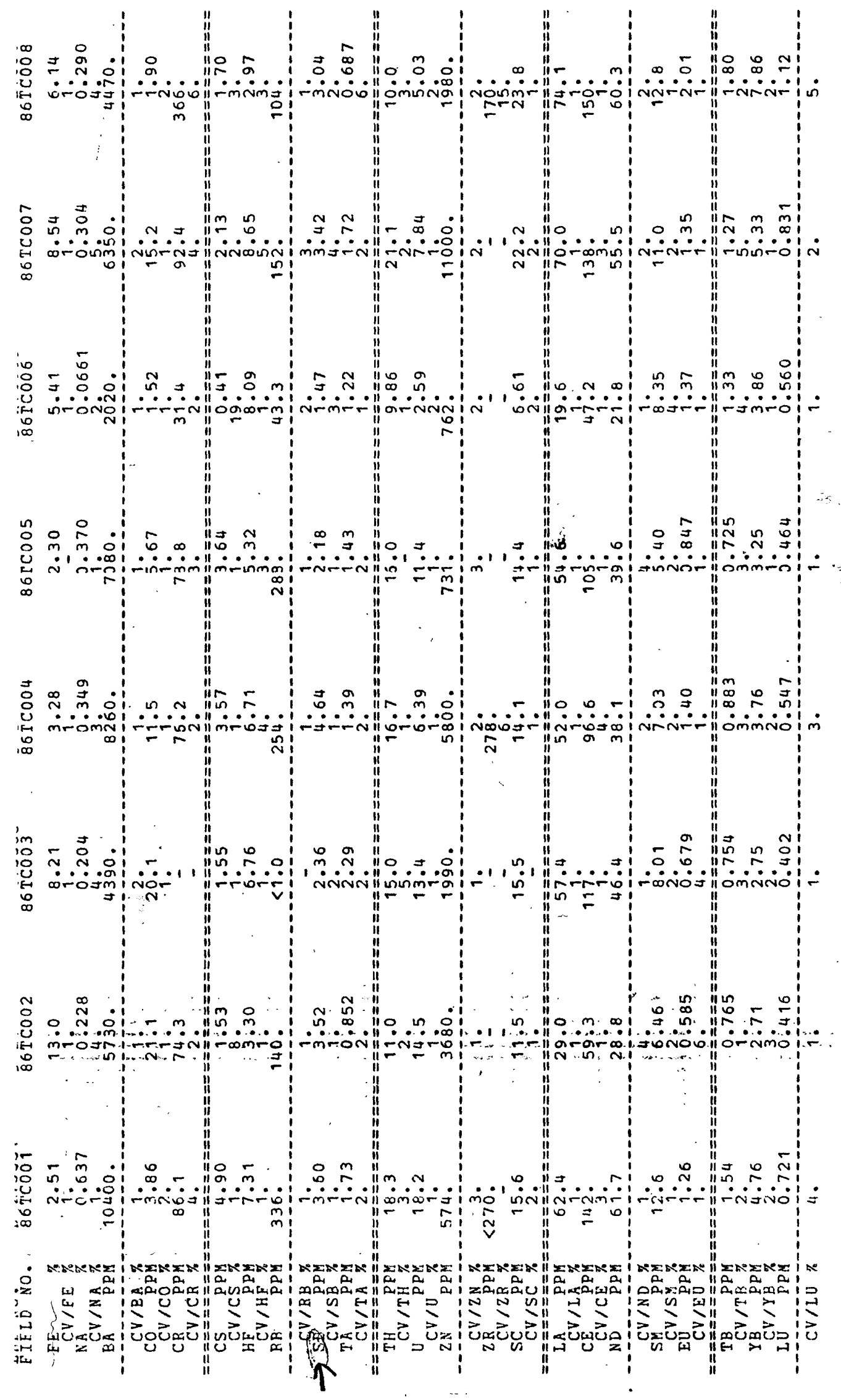




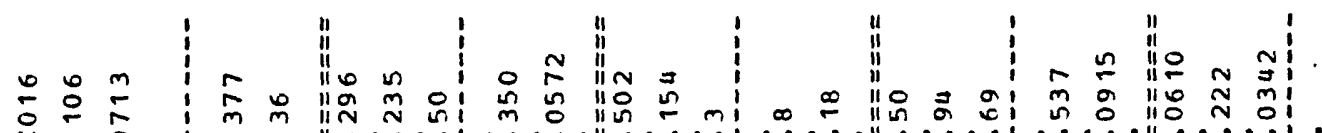
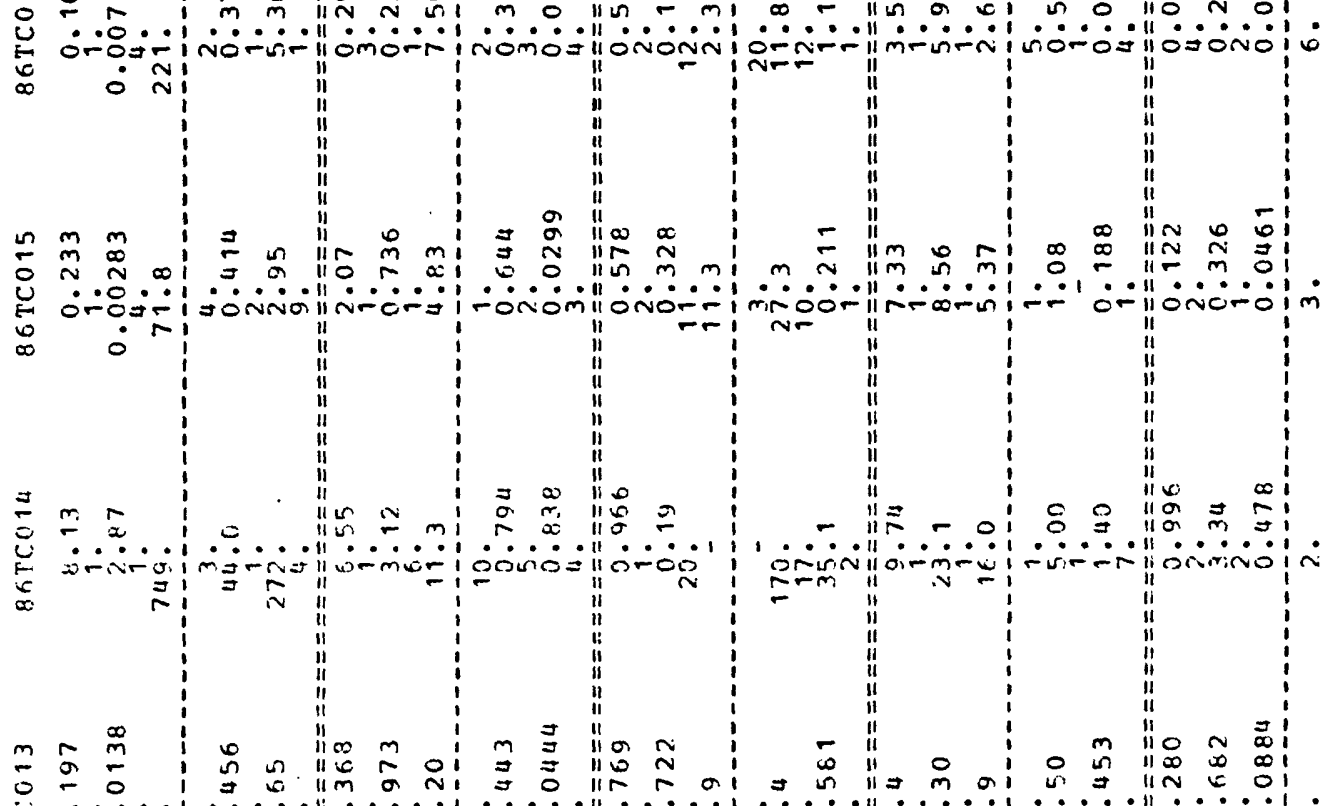

0

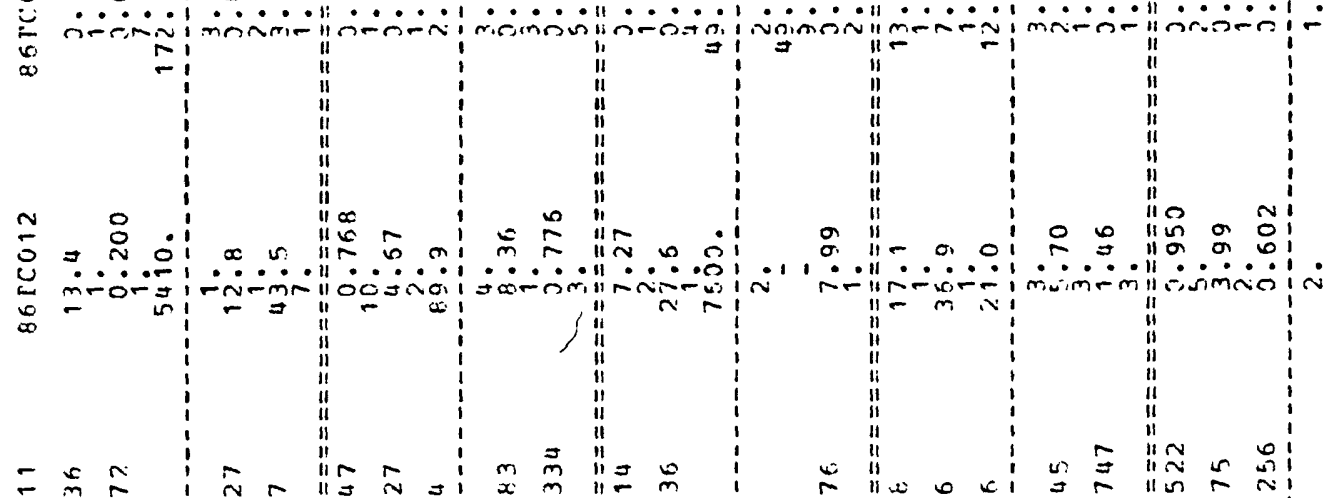

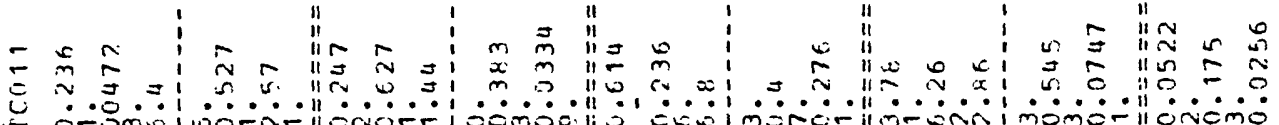
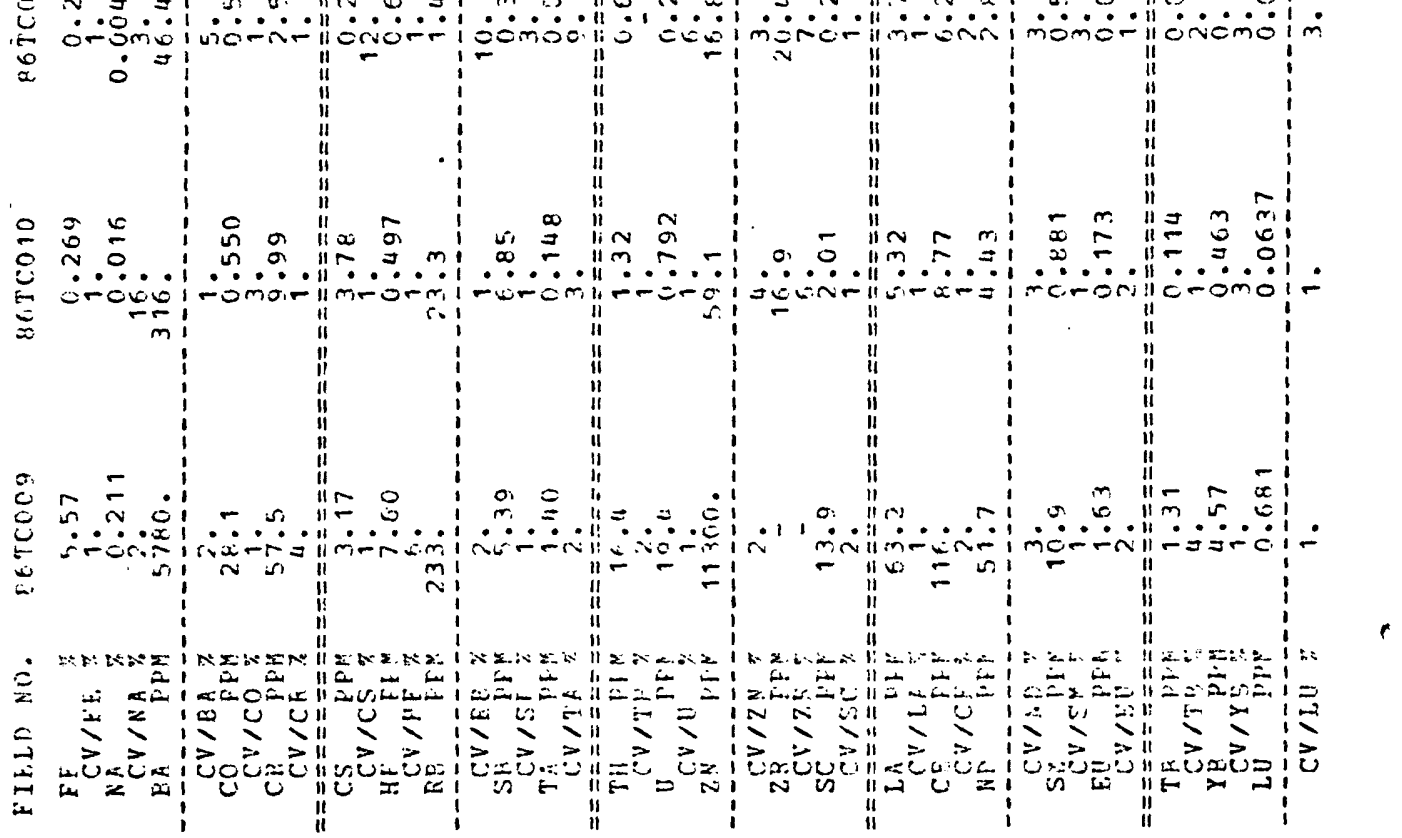


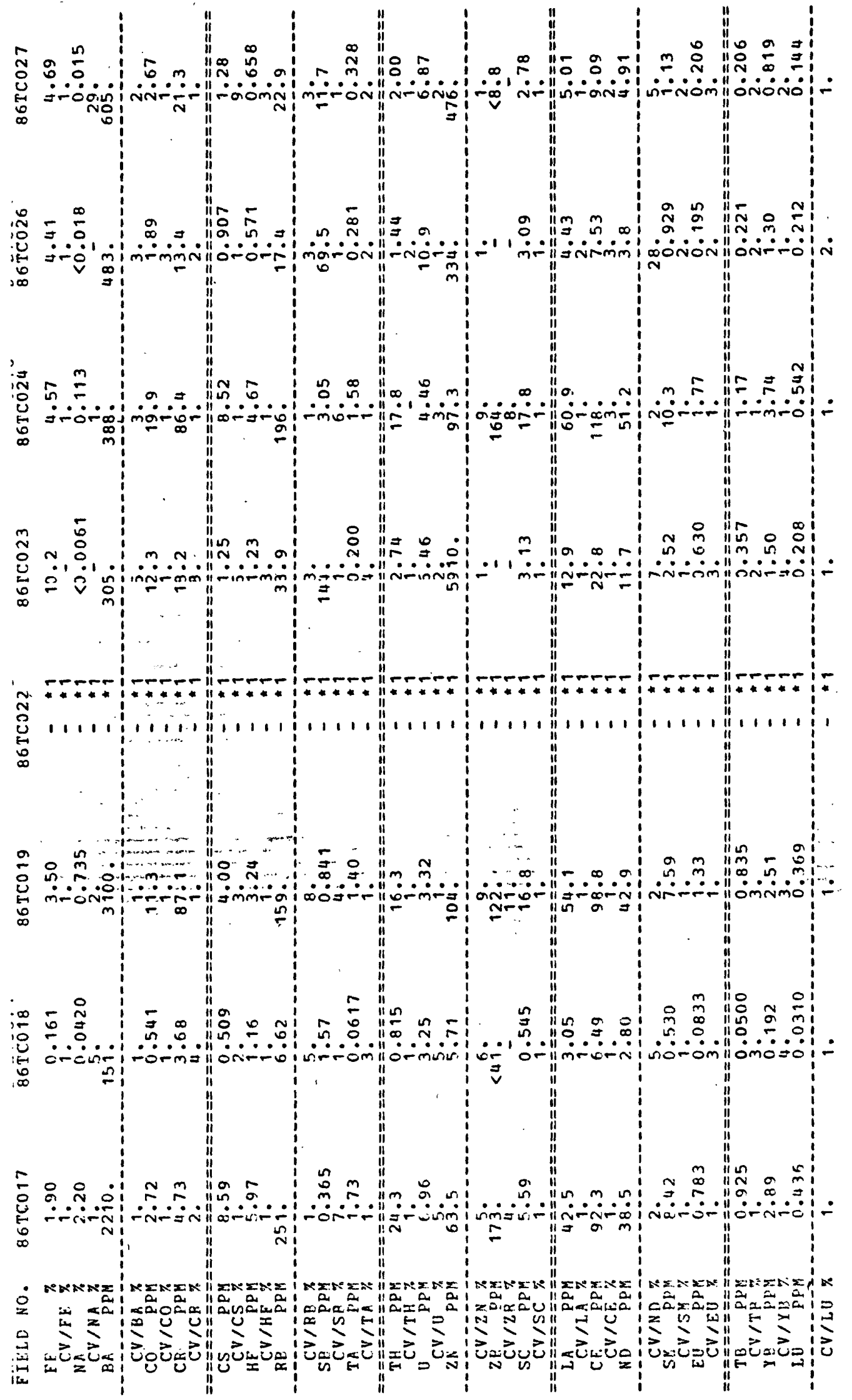




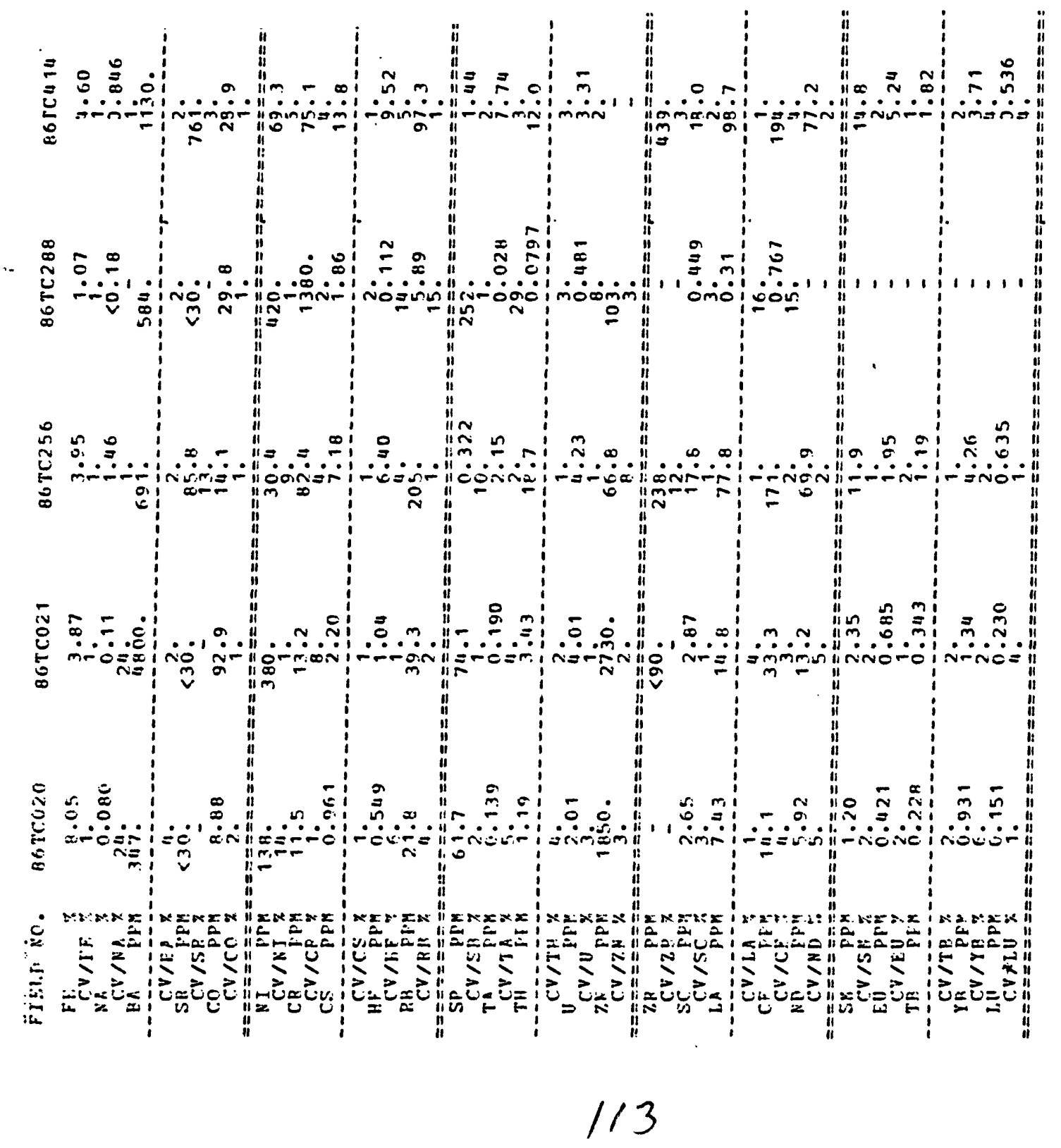




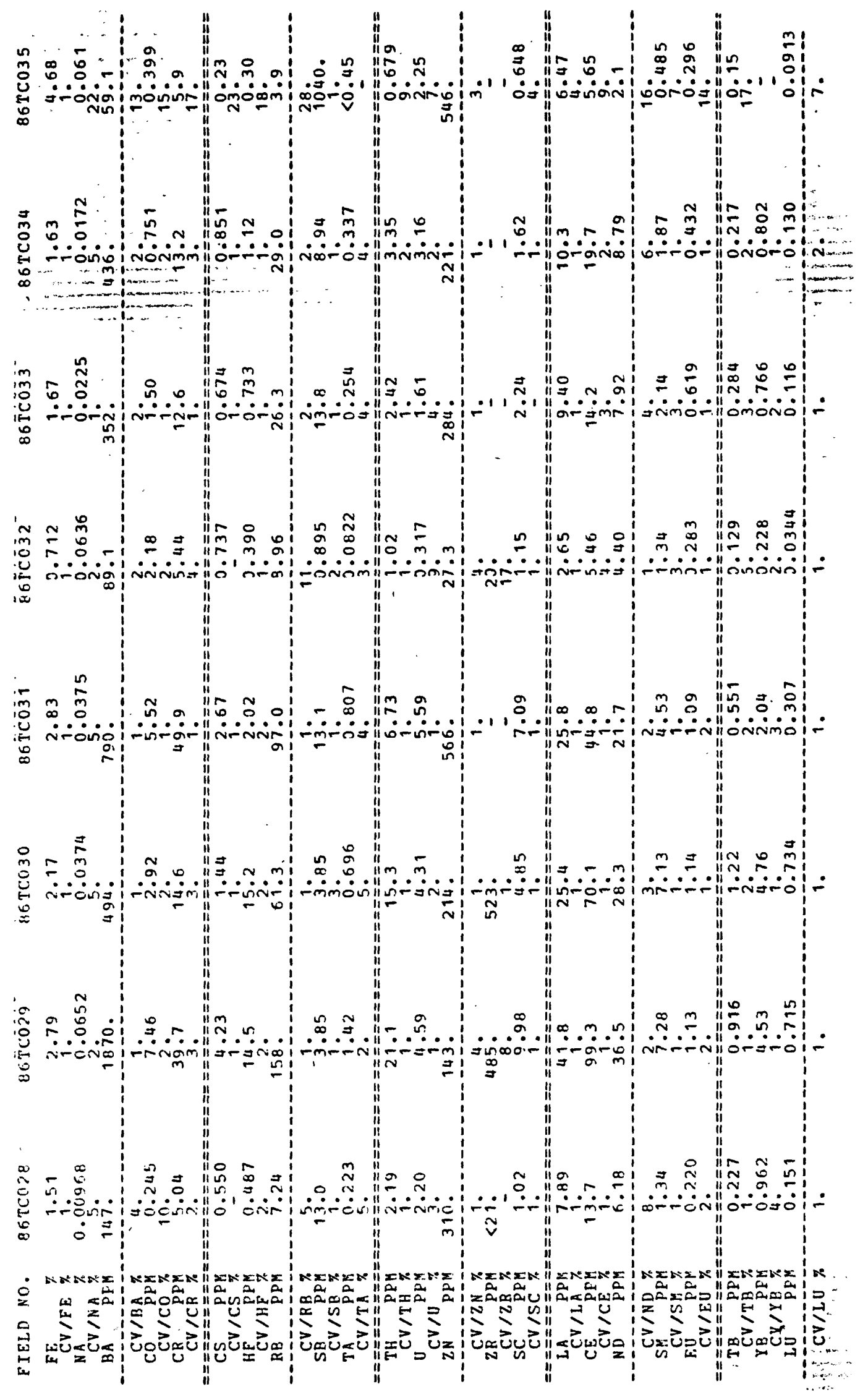




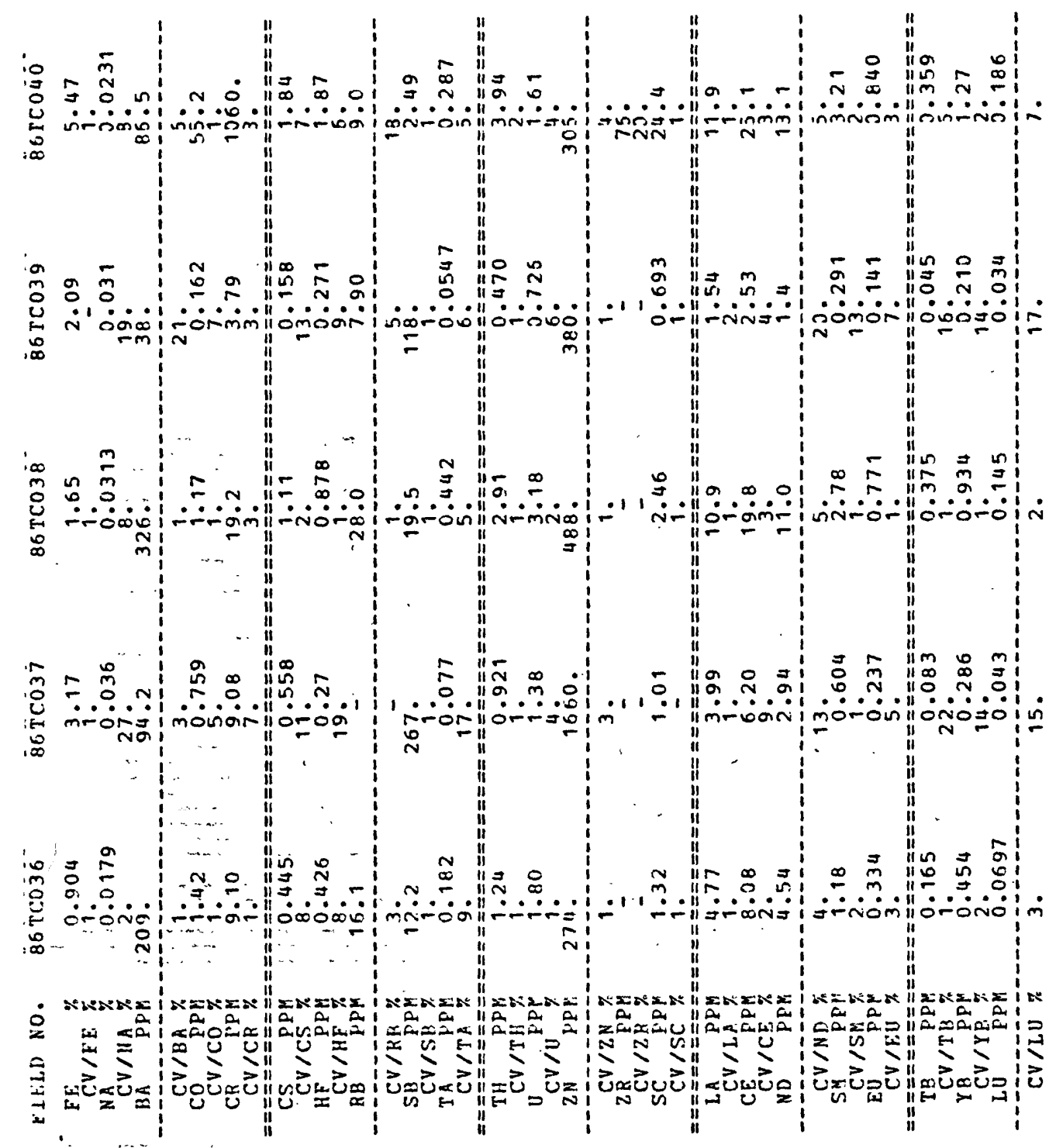




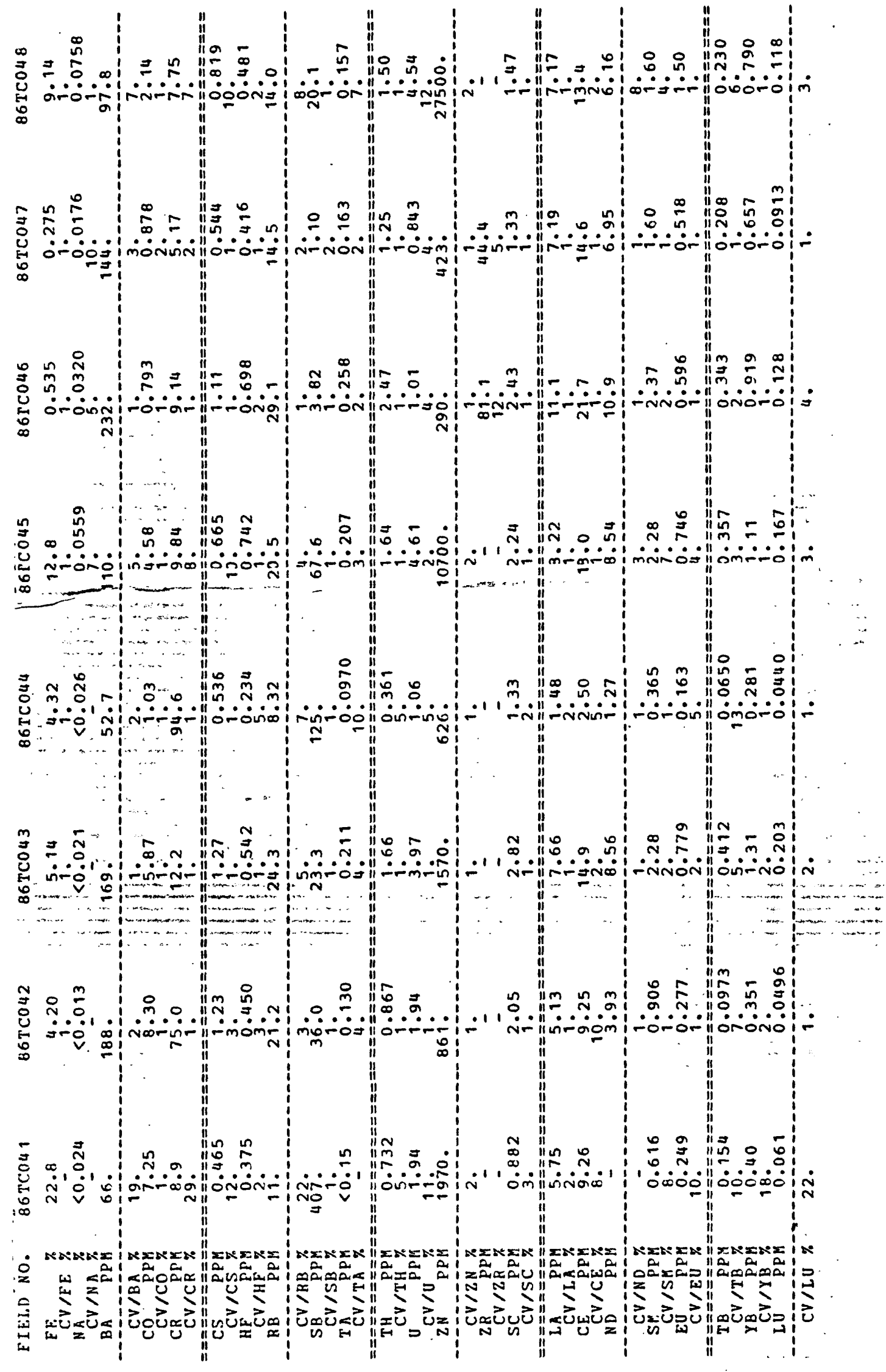




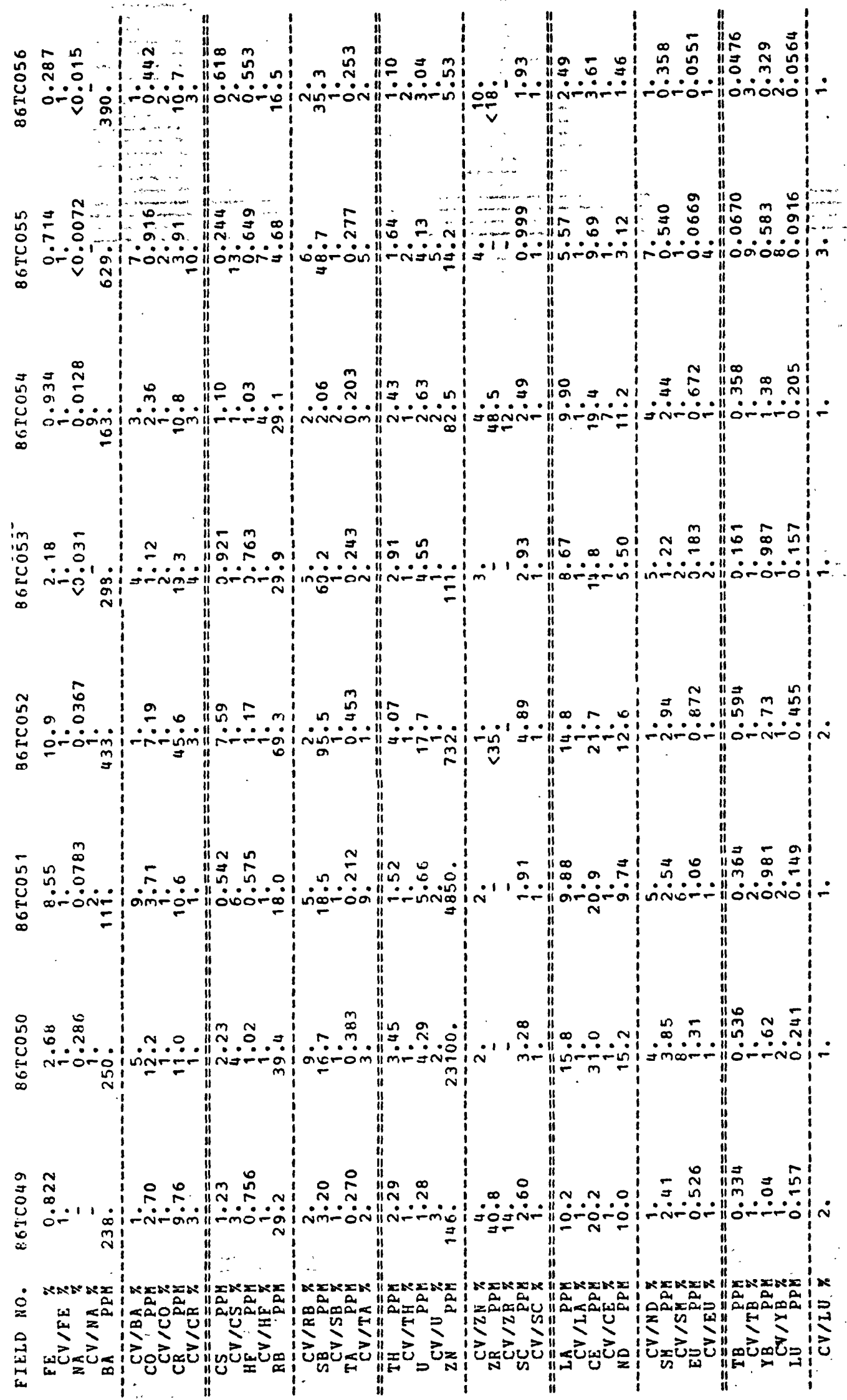




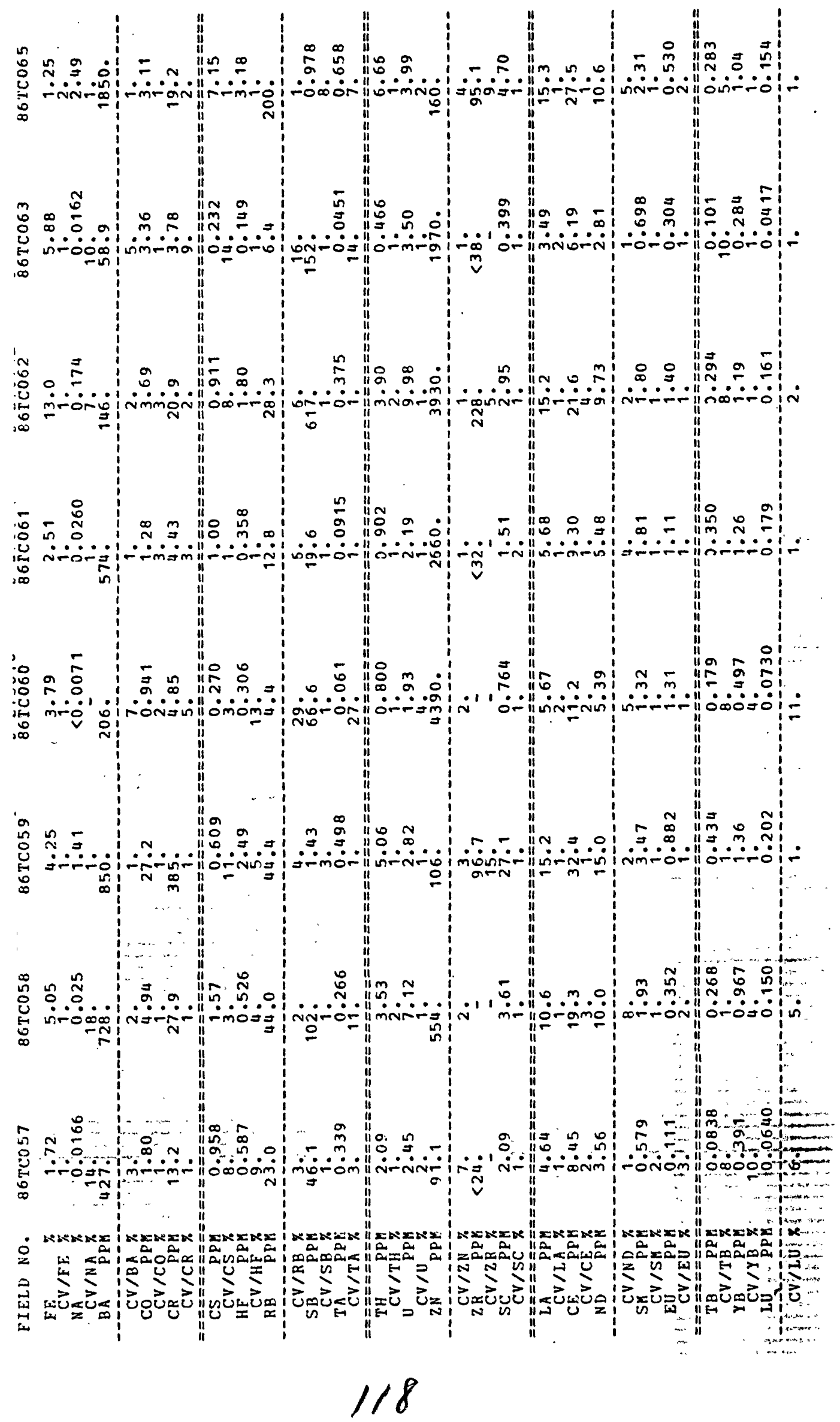




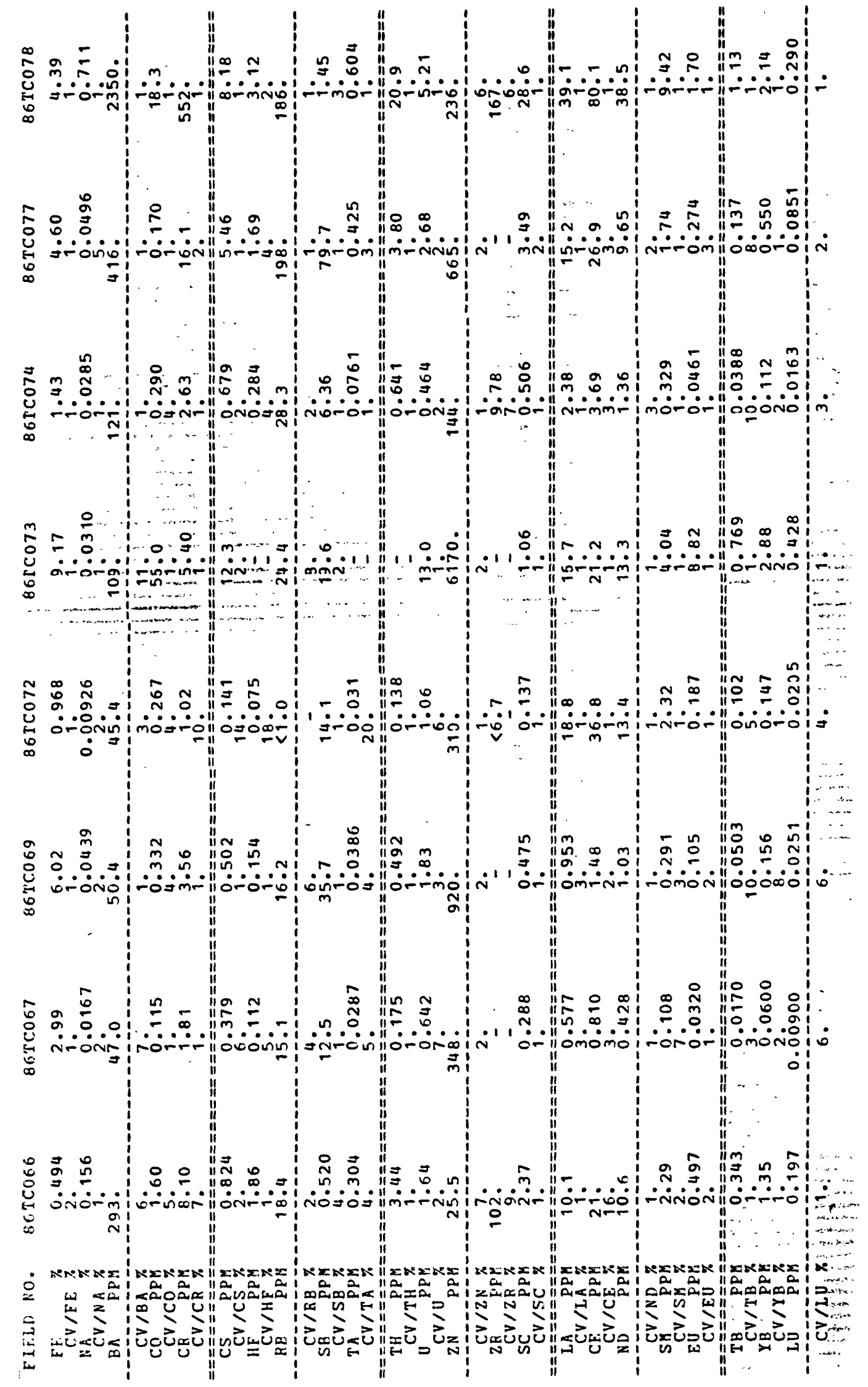




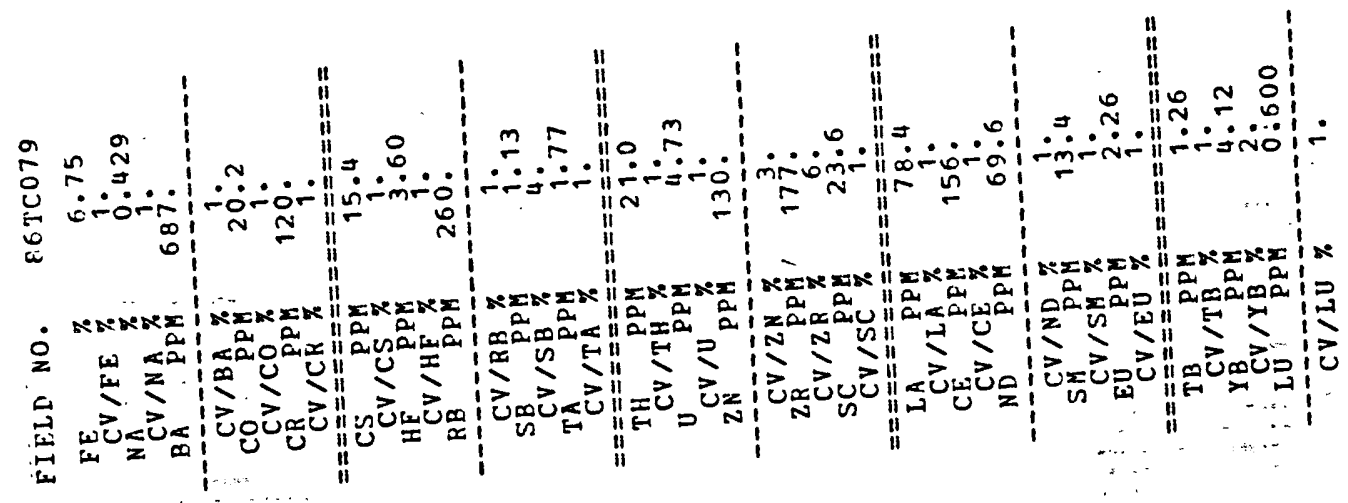




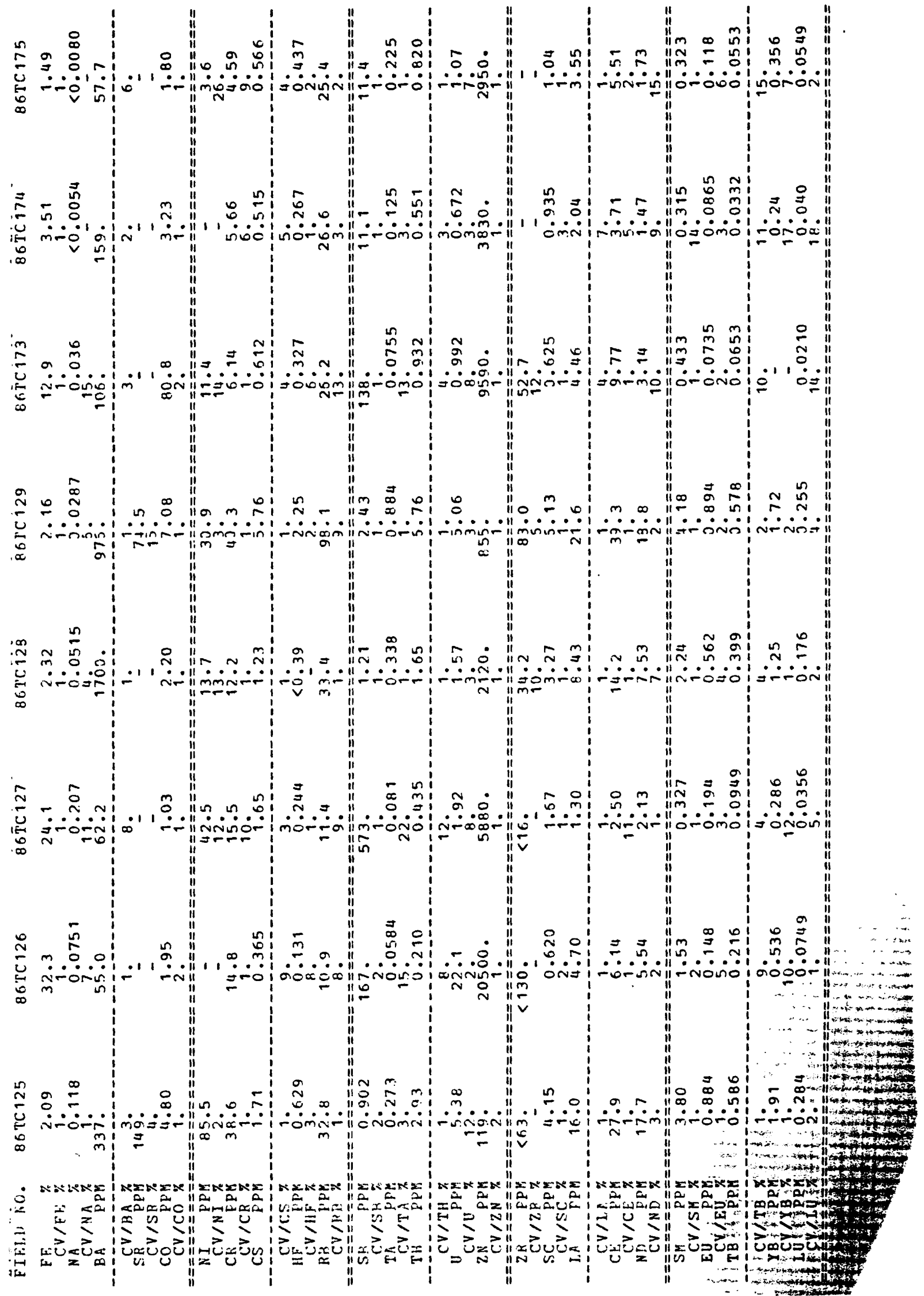

122 


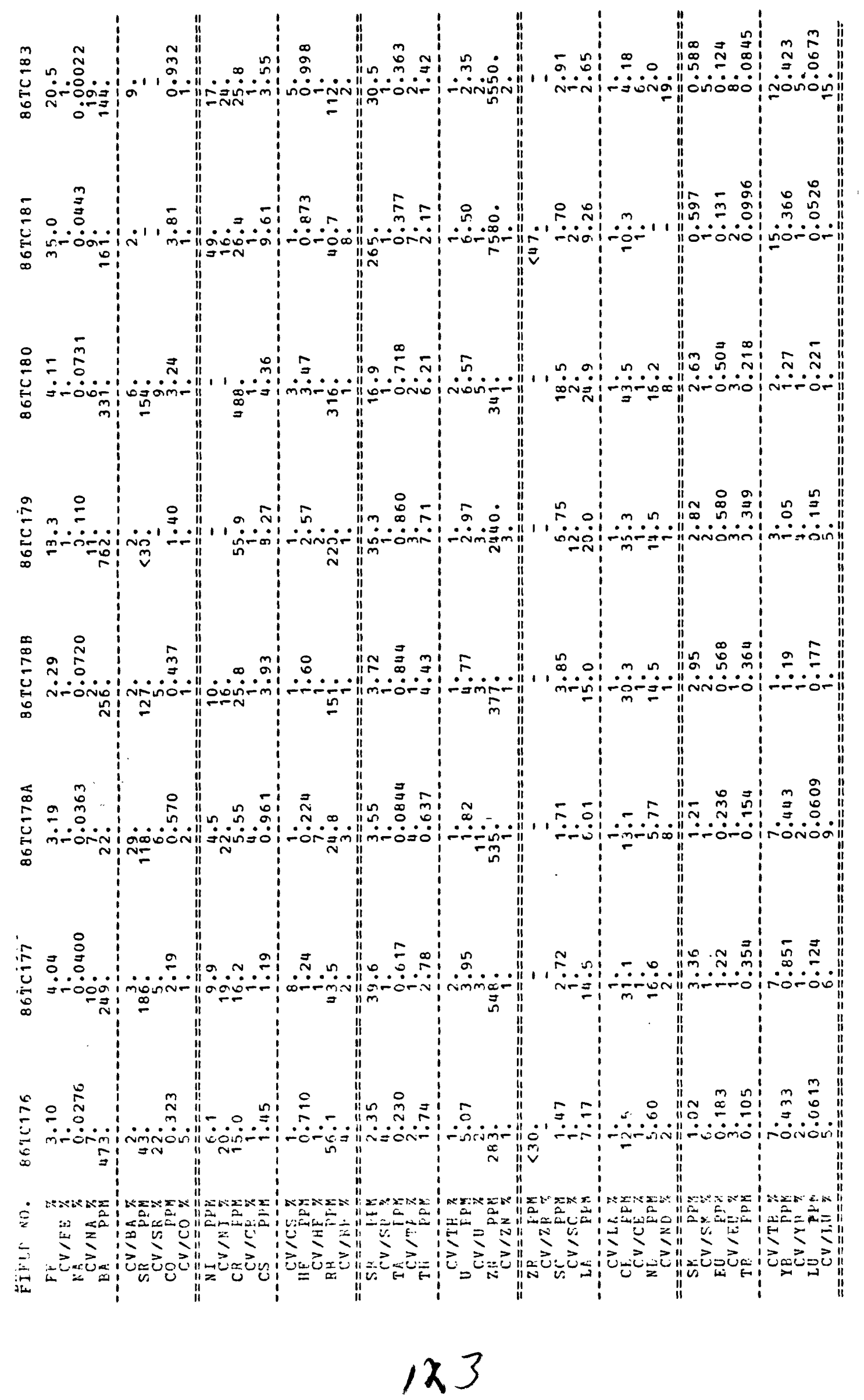




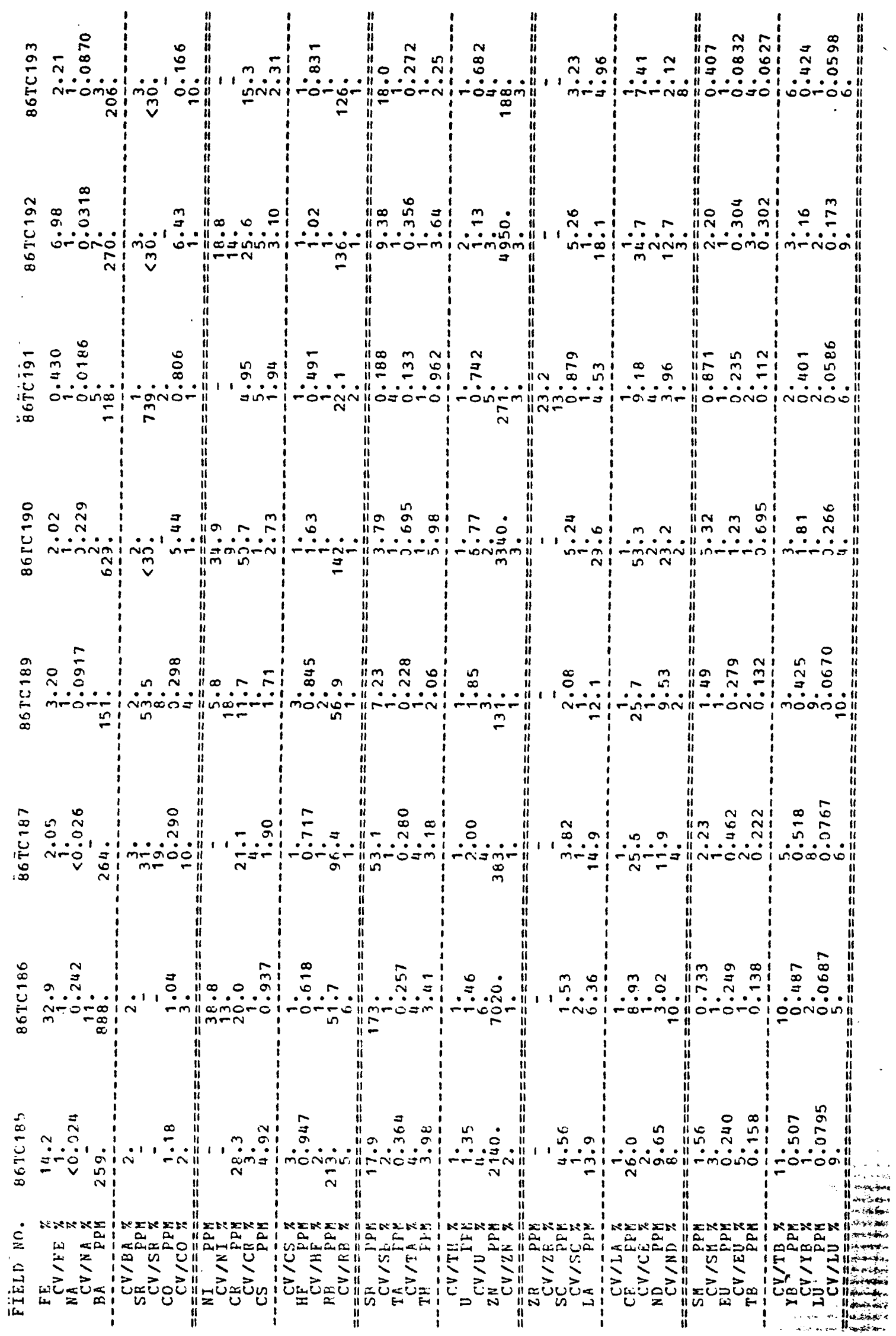



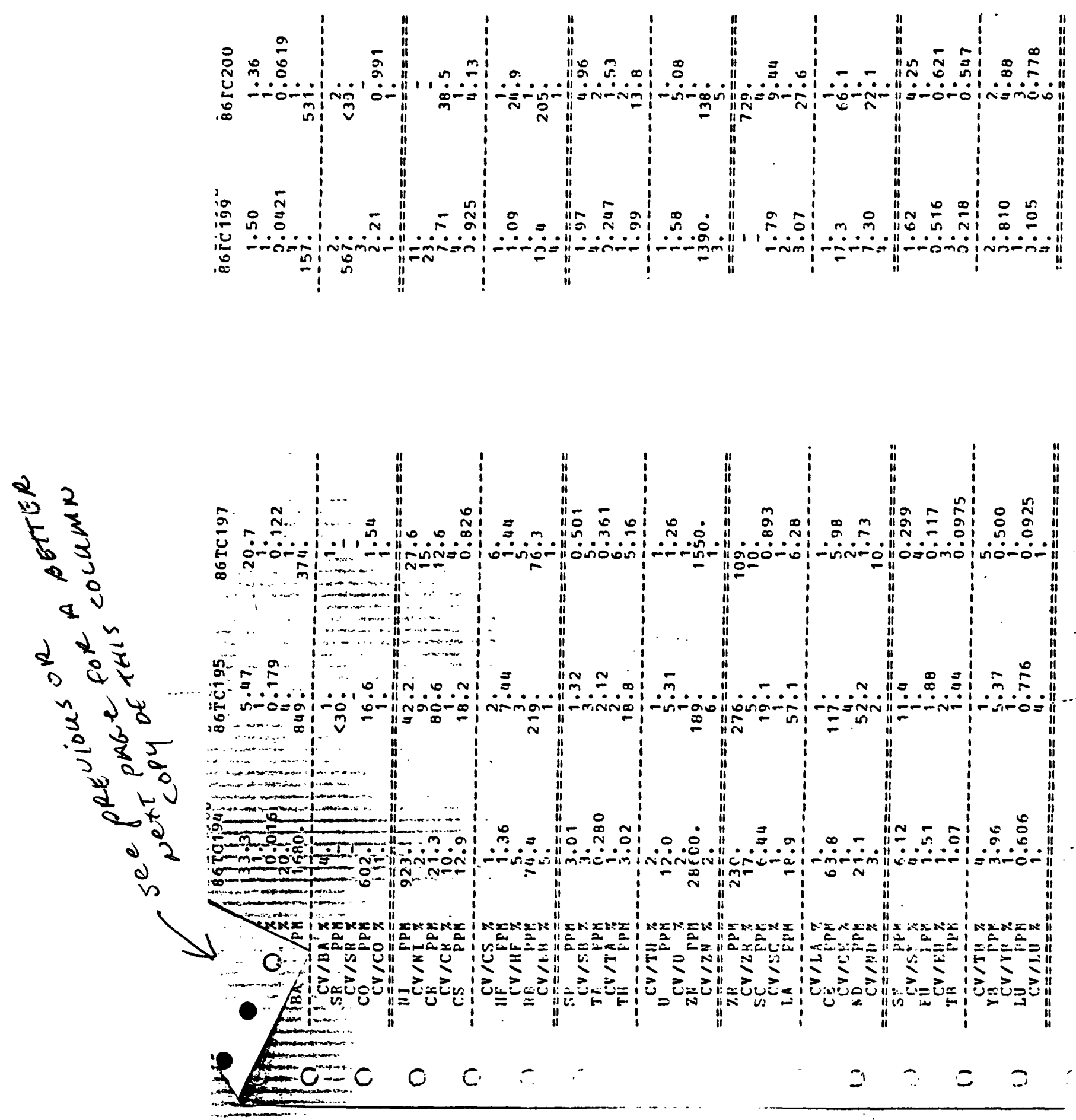

125 


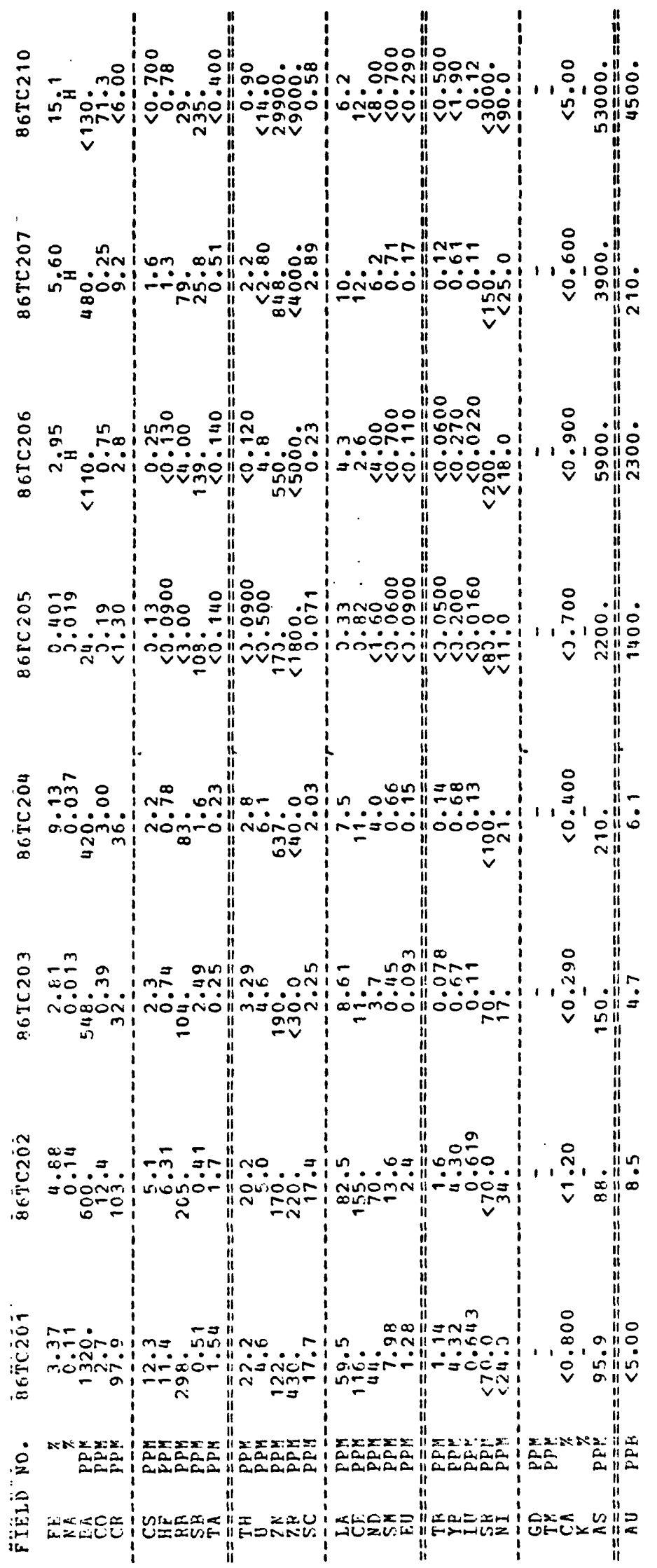




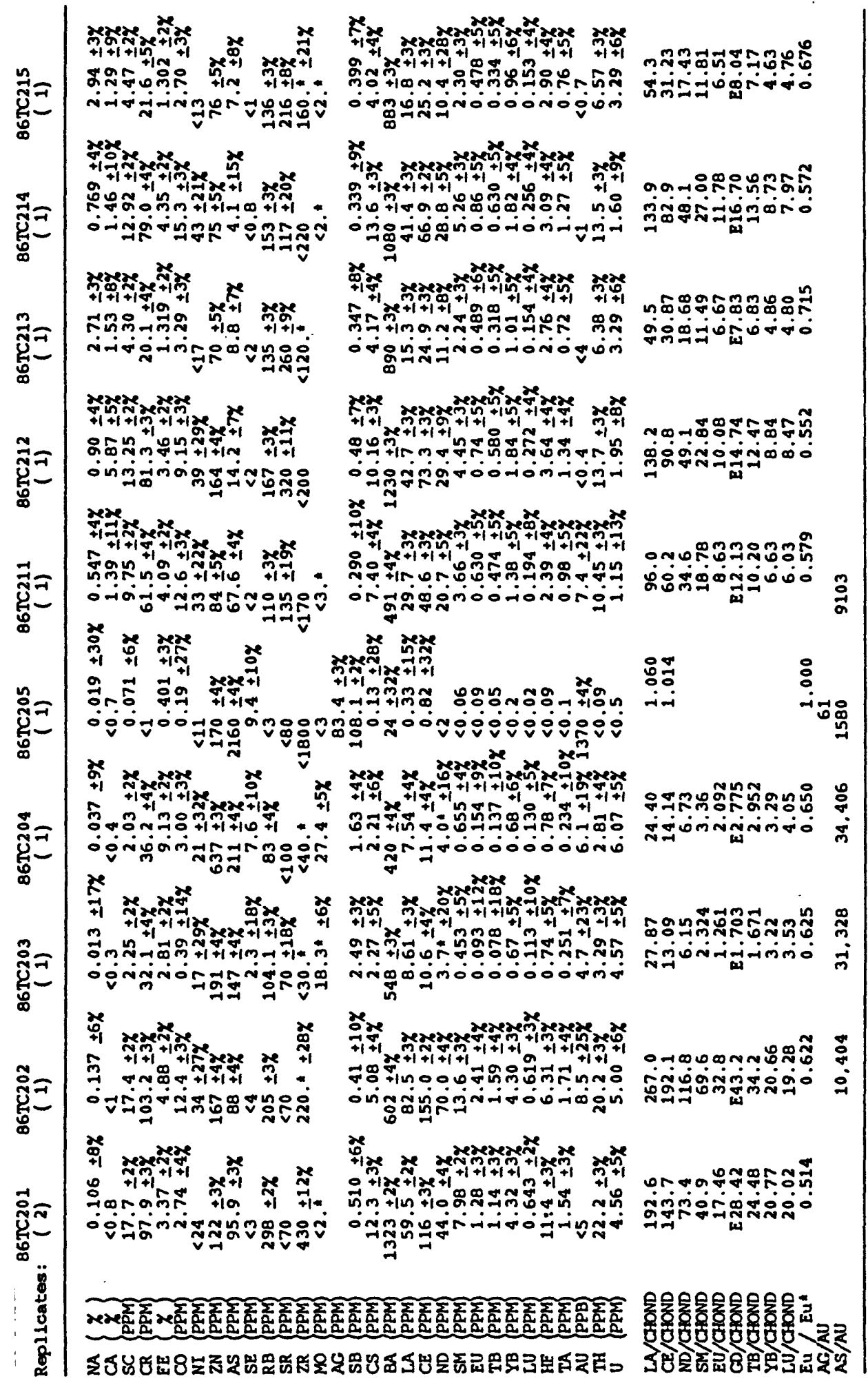

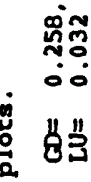

on

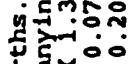

郭副击

कृ

t5

वैं

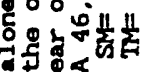

1 的

3 c ᄃํ...

ํํำ

这

ที

รับ

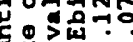

8 ํㅏㅇ

ธ들

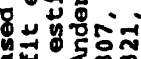



$5=0 \%$

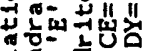

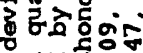

०ण

to엉

돈

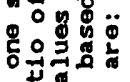

o $>$

1.

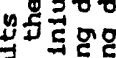

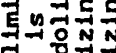

ㅎํำ

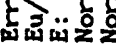




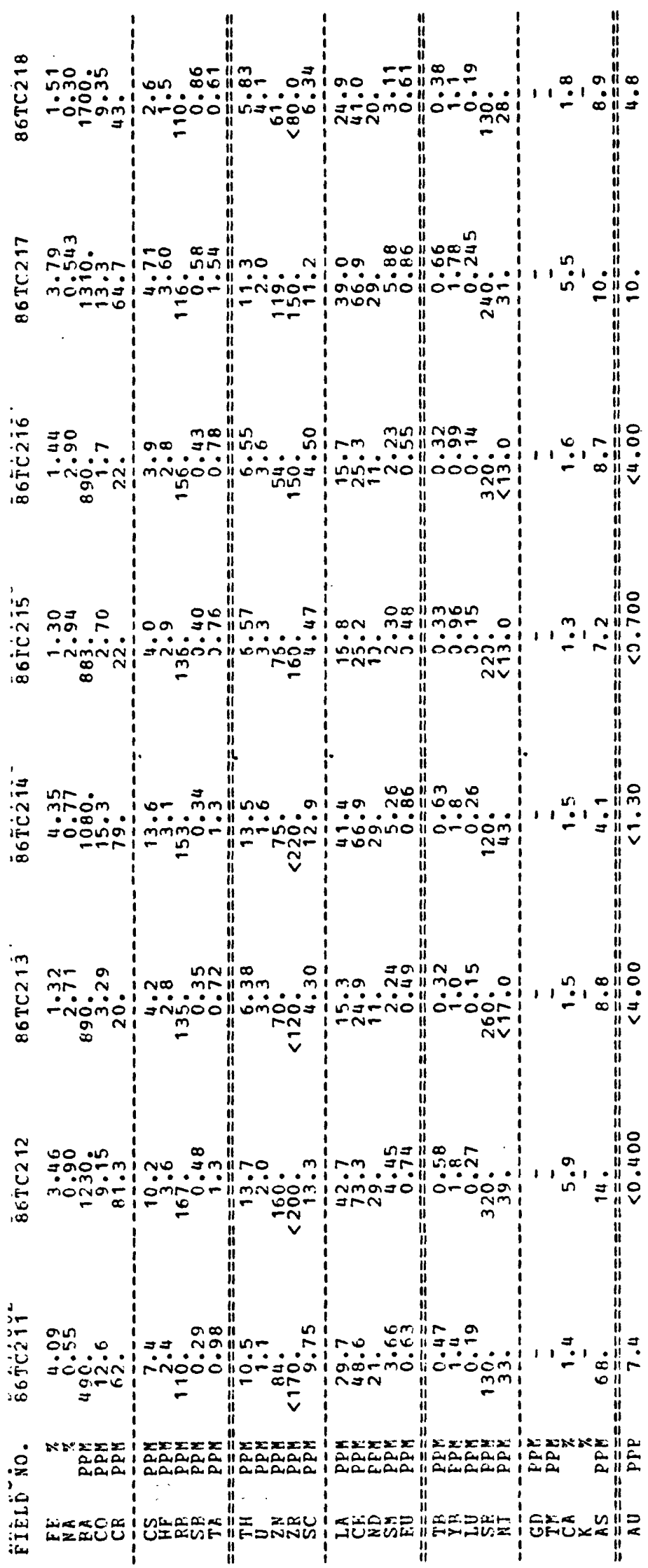




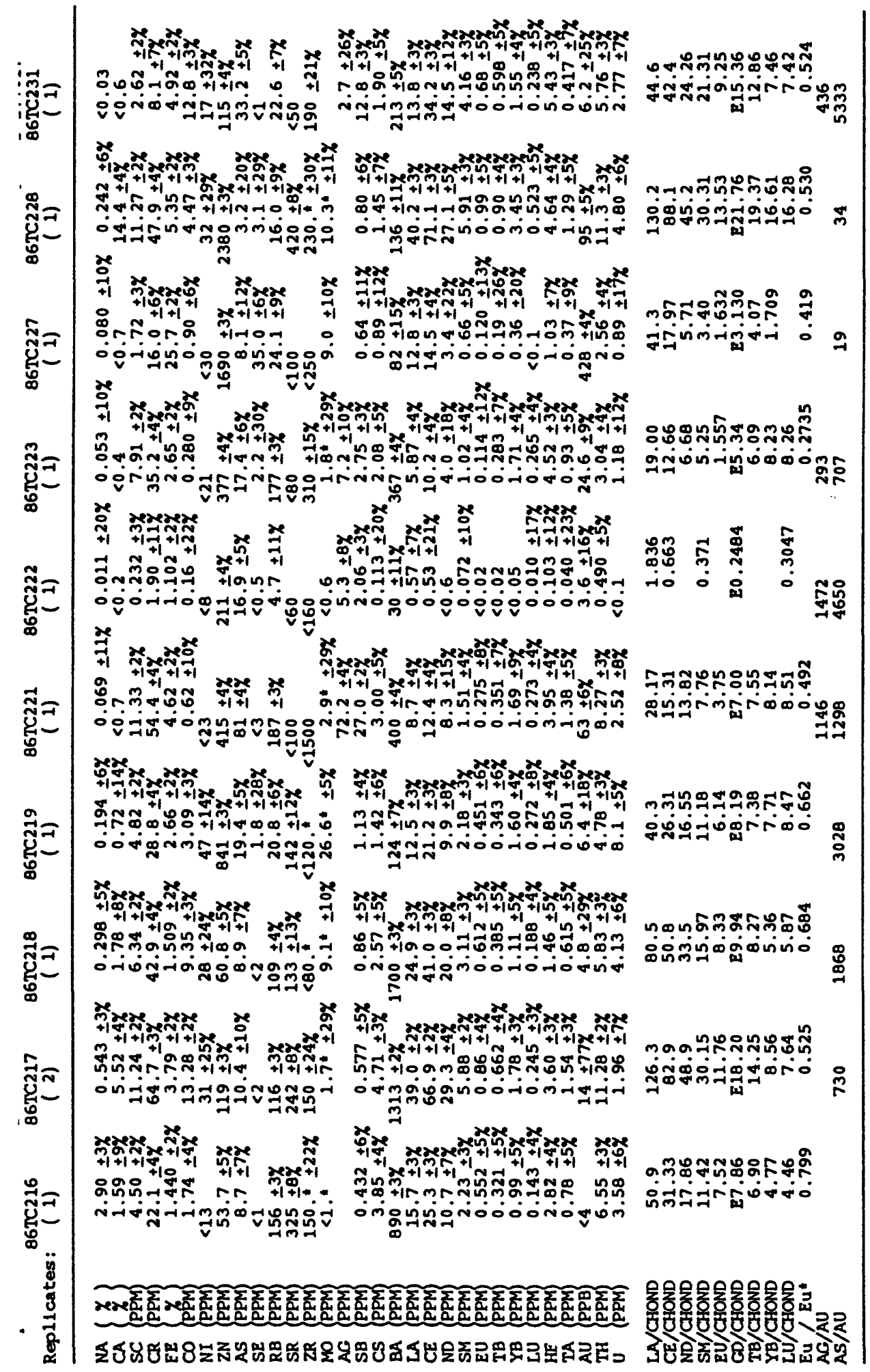

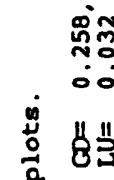

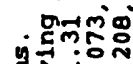

दें००

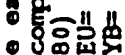

\% rin

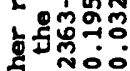

额

ช ธ

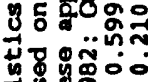

击

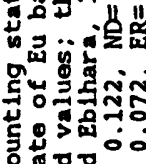

8 罗实

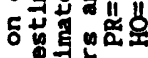

舟落云

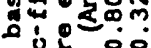

ธำ 행

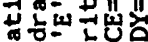

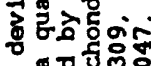

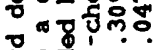

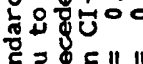

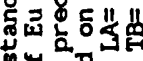

ตำ

논

国要

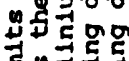

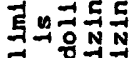

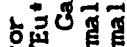

率寻前完完

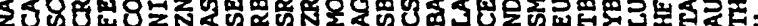




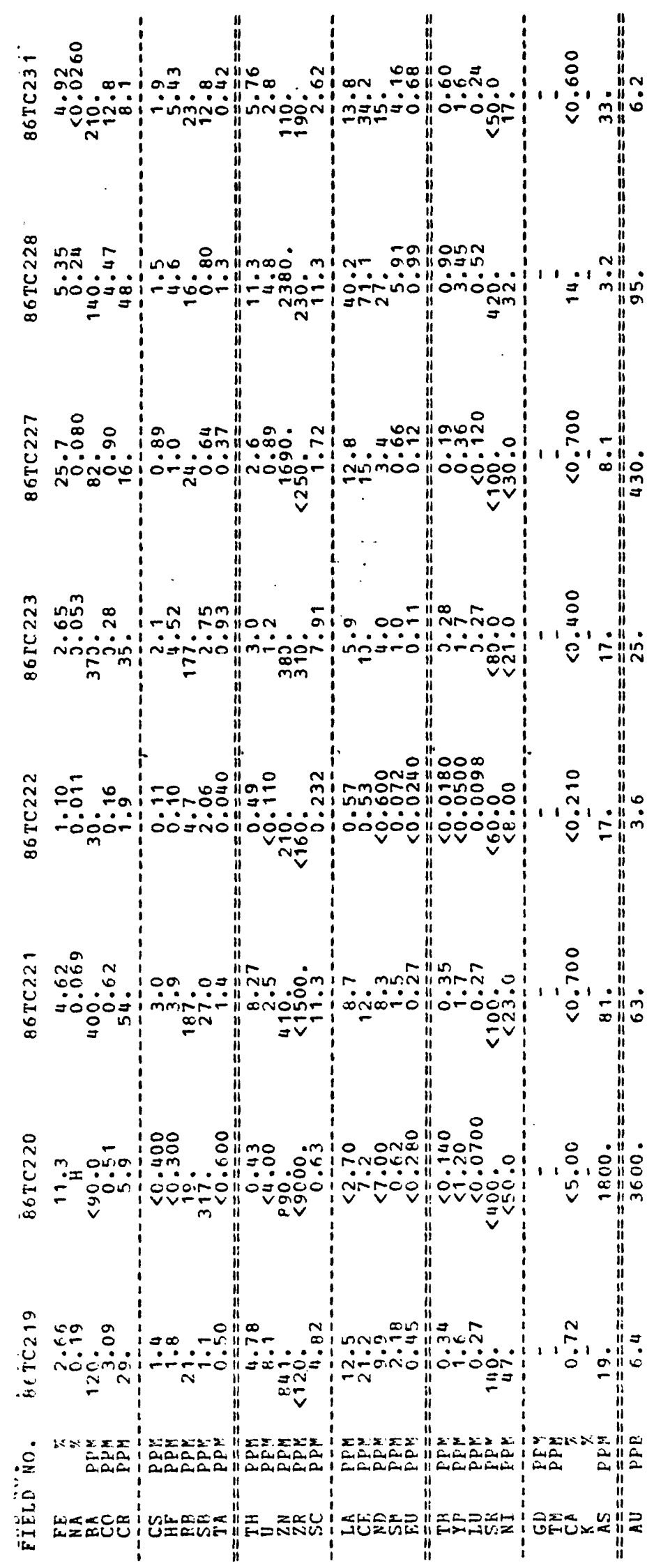




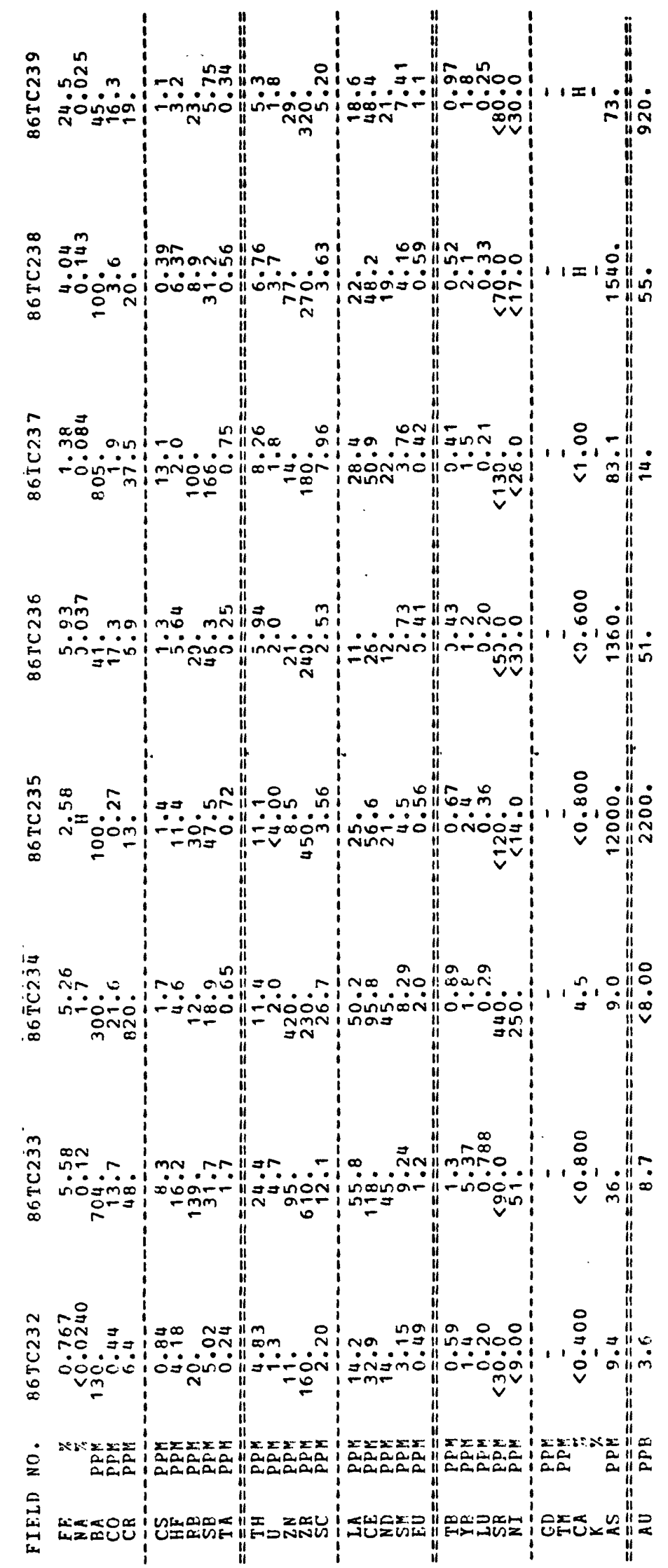




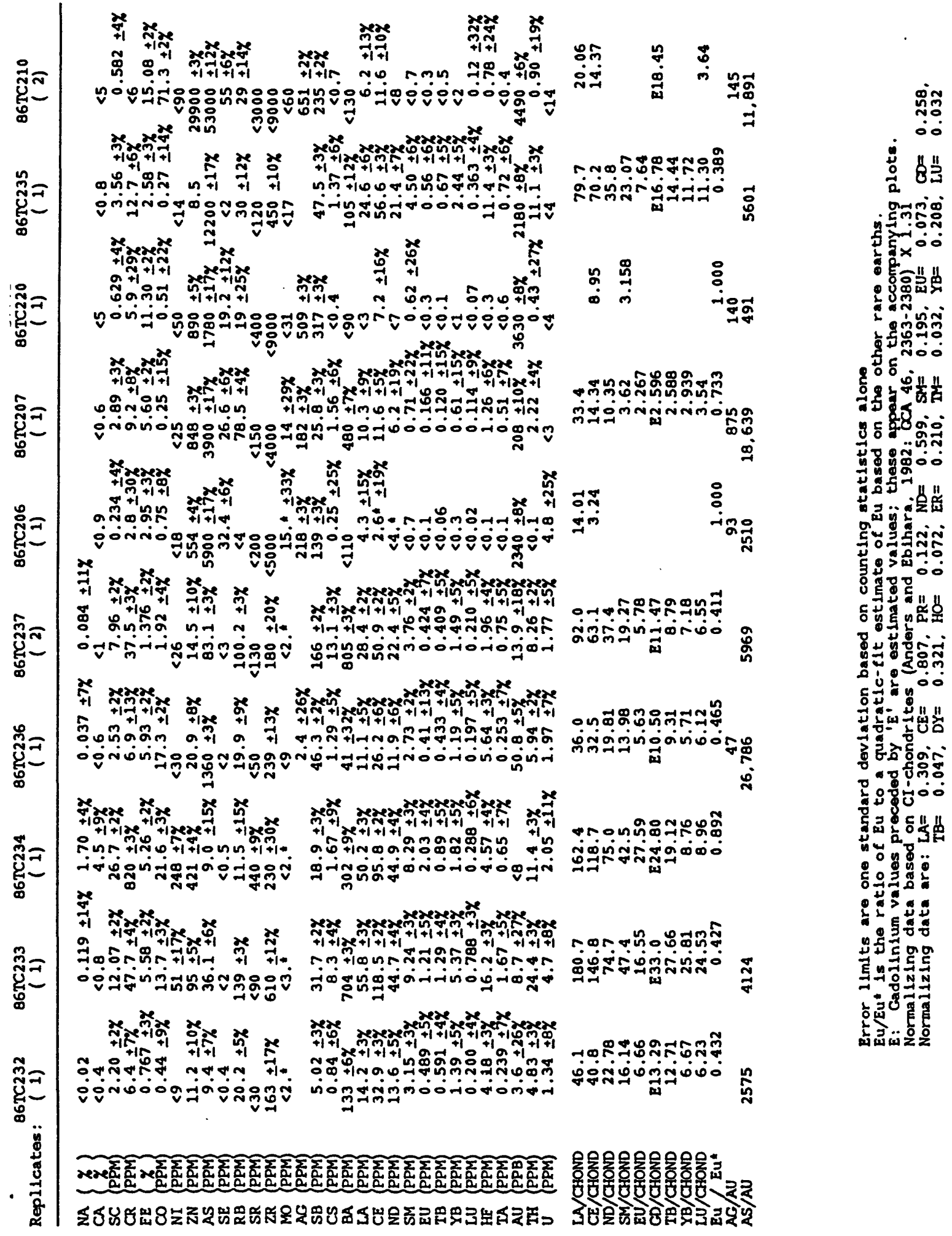



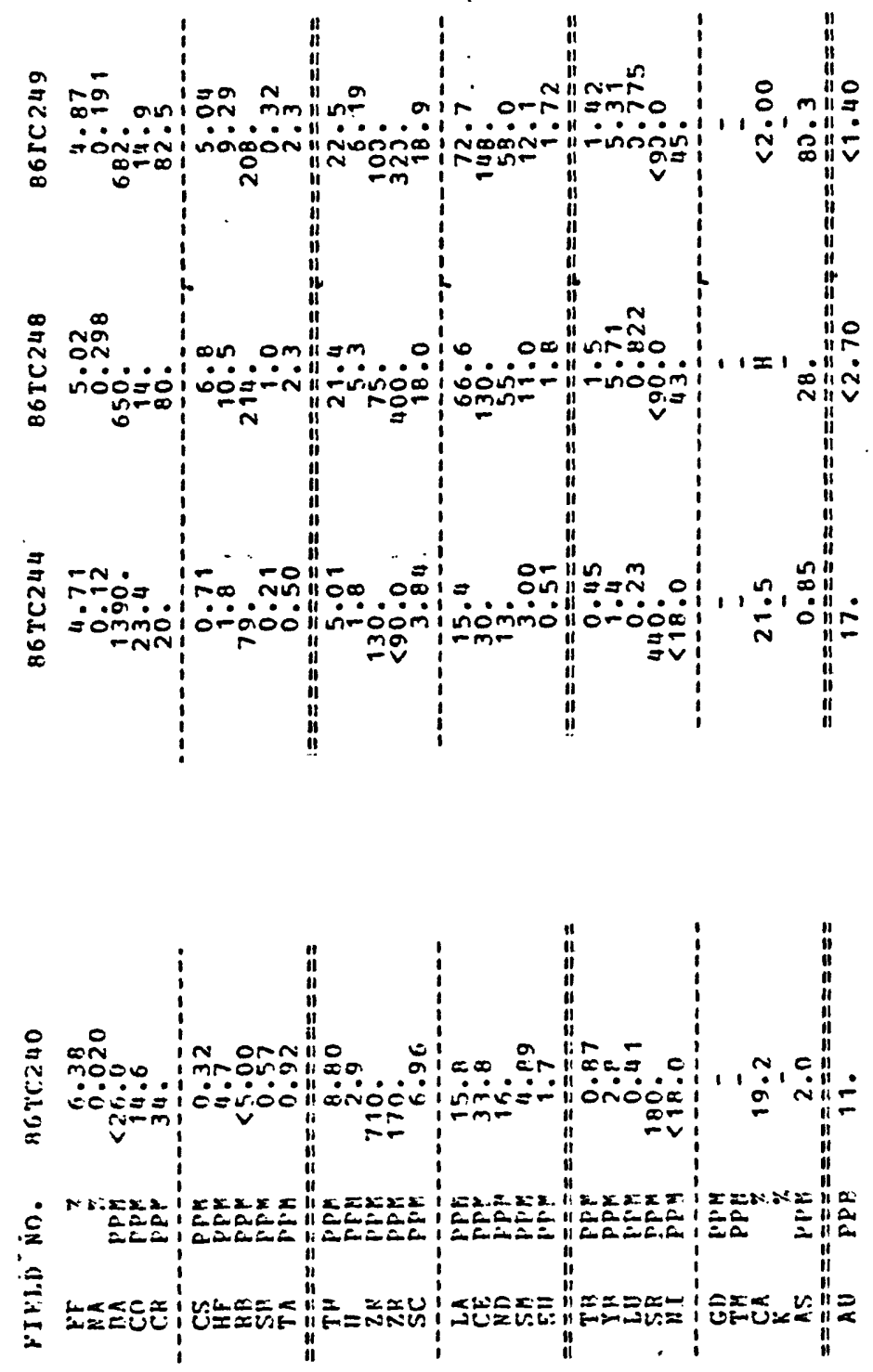


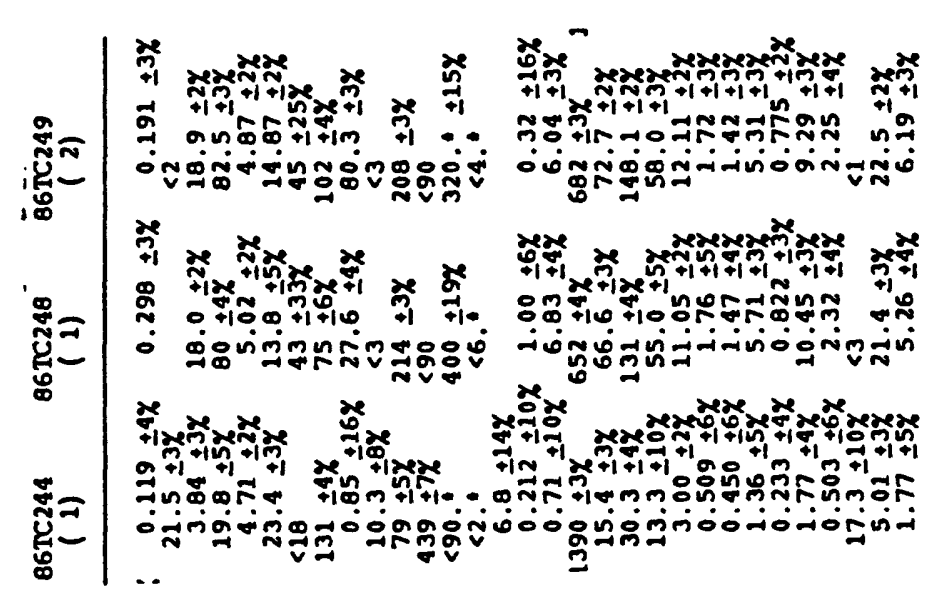

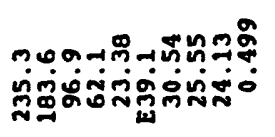

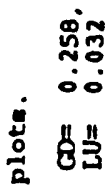

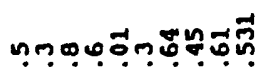

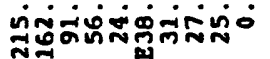

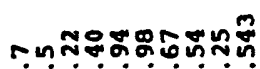

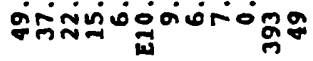

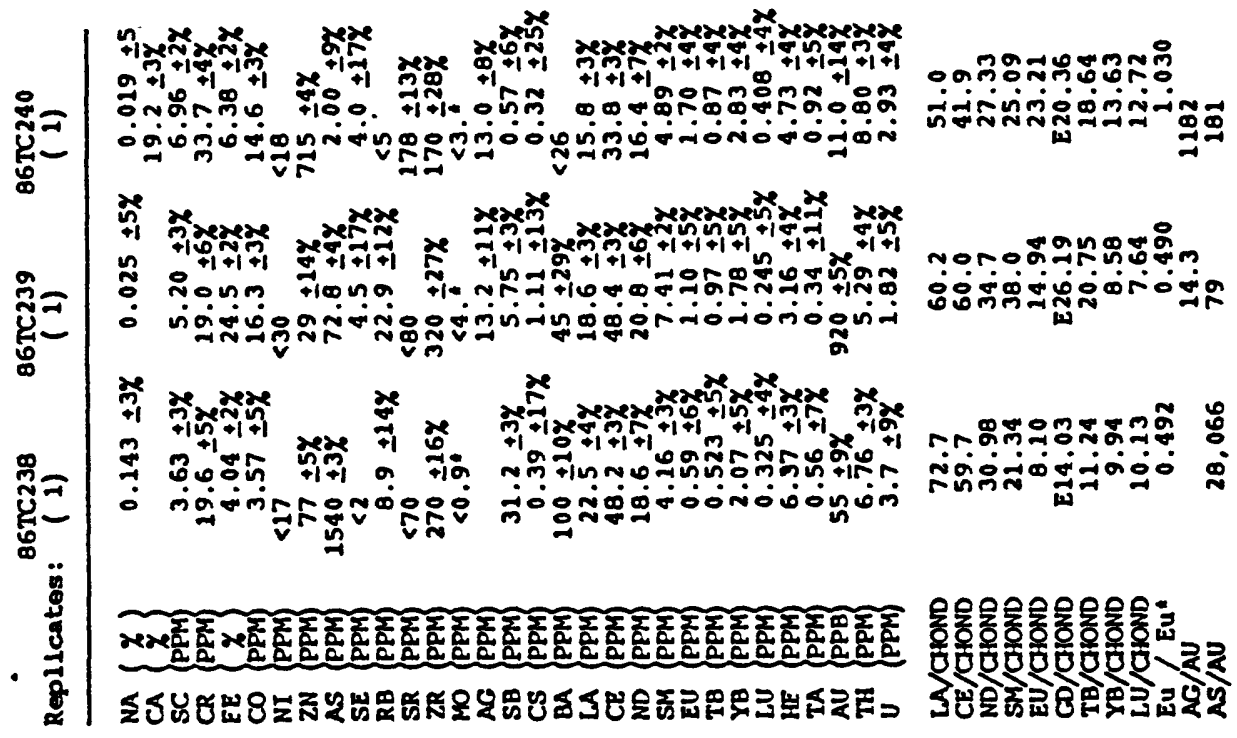

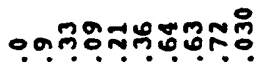

ㅇำ

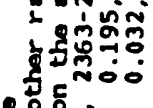

8 :

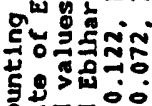

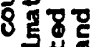

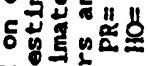

总㚜运

年

年

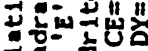

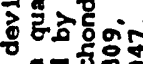

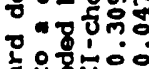

通连它只

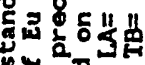

눙

ส.

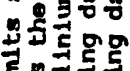

트므ㅇㅝㅠ

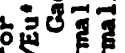

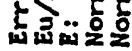




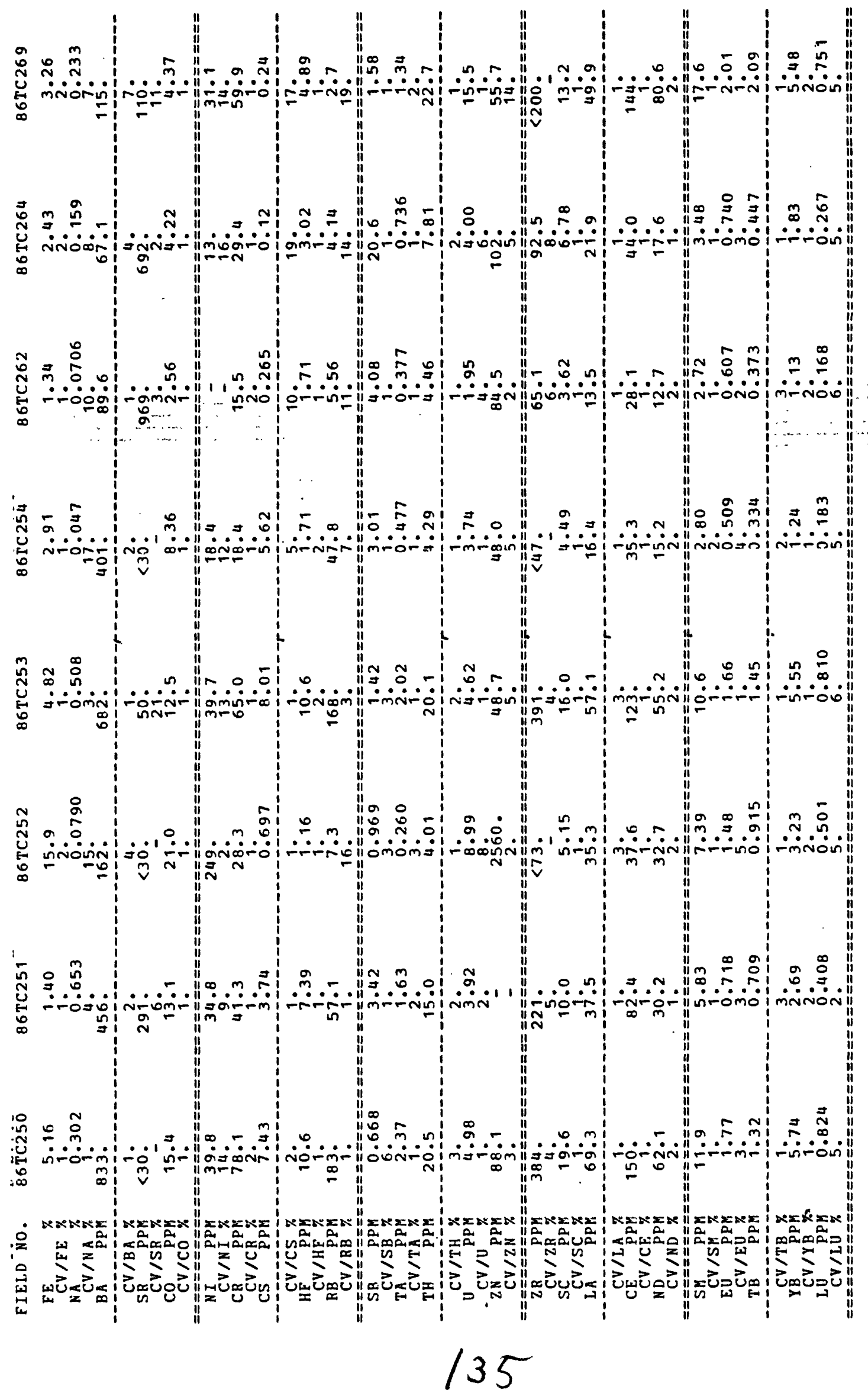




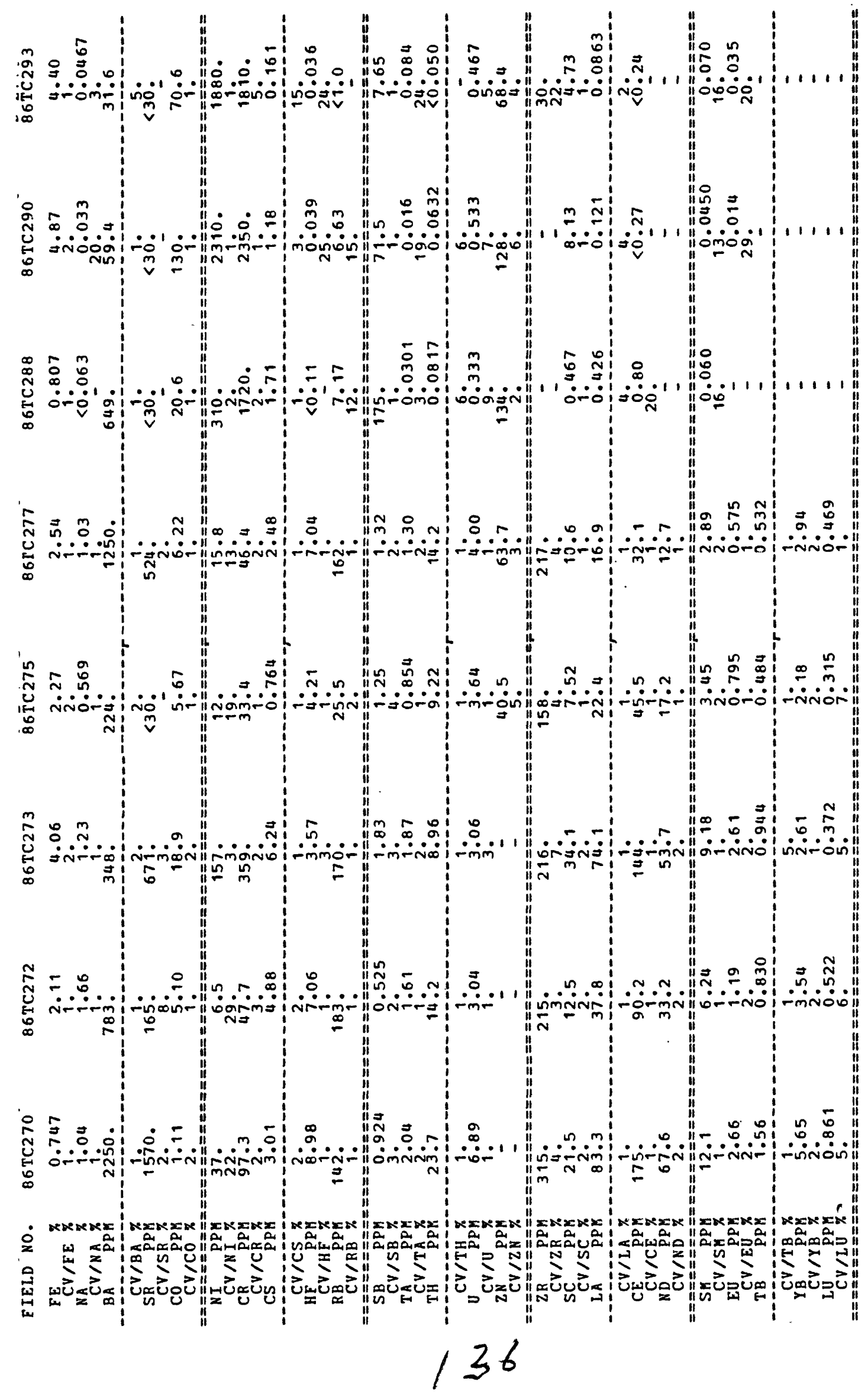




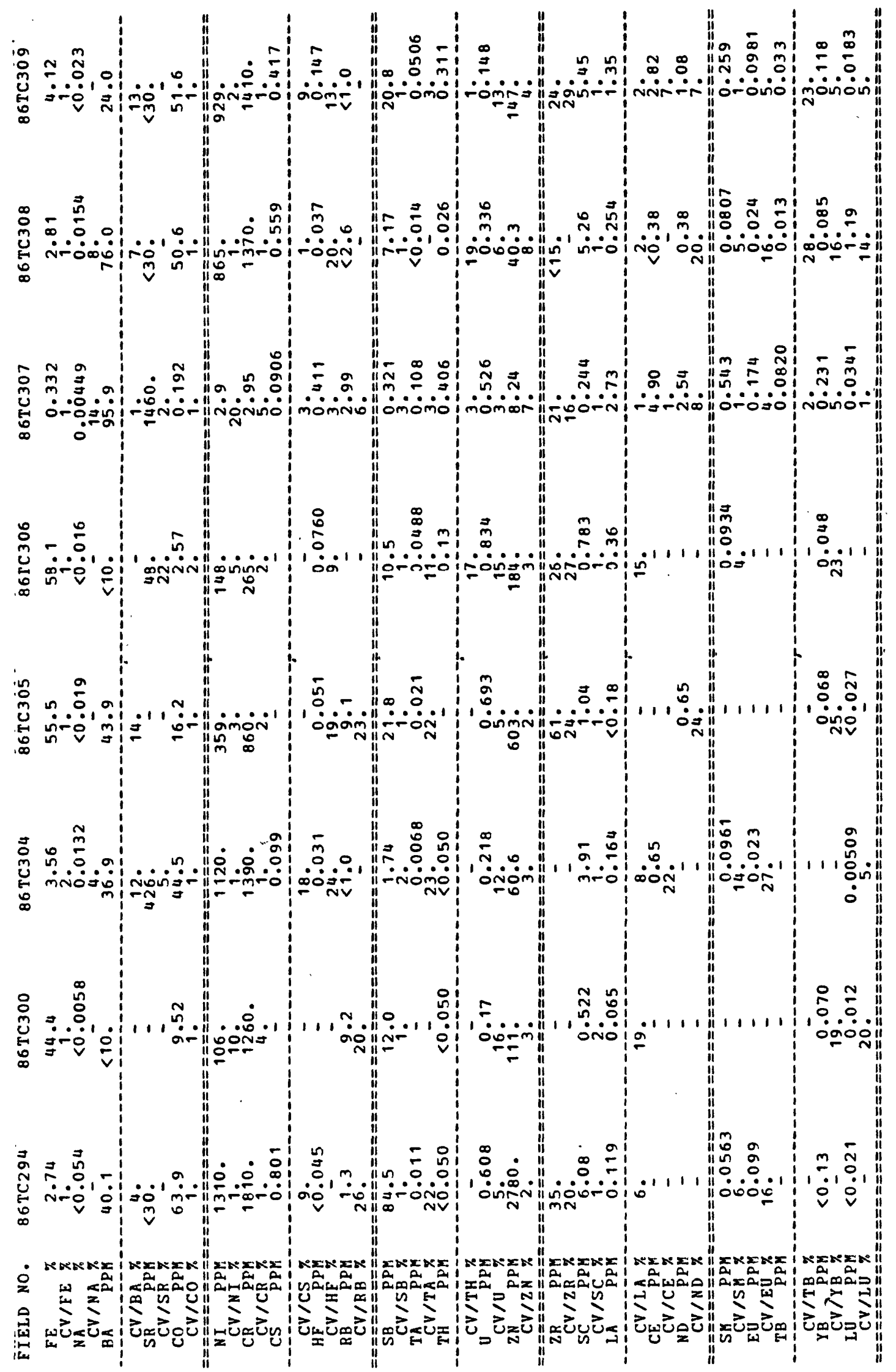




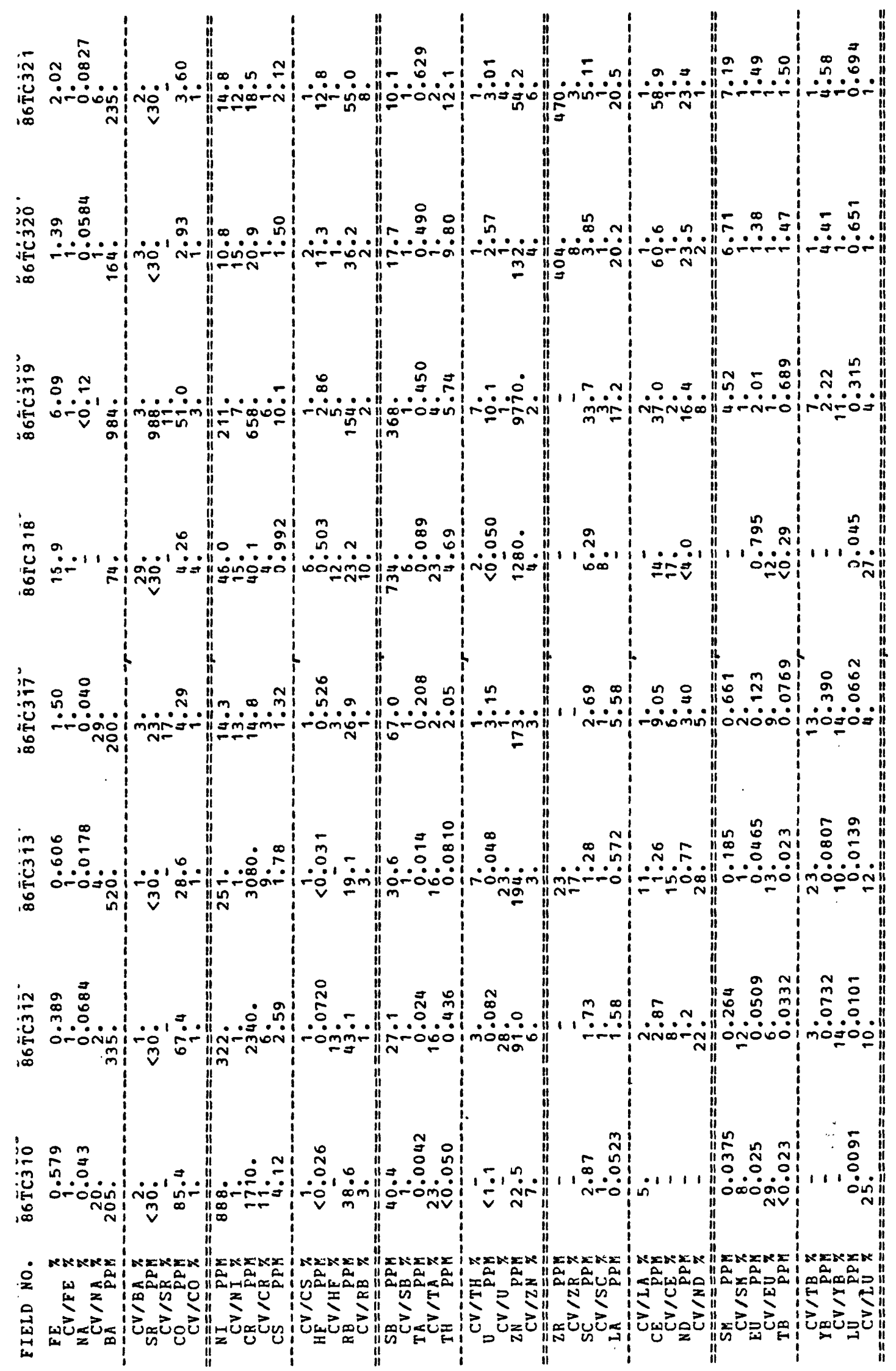




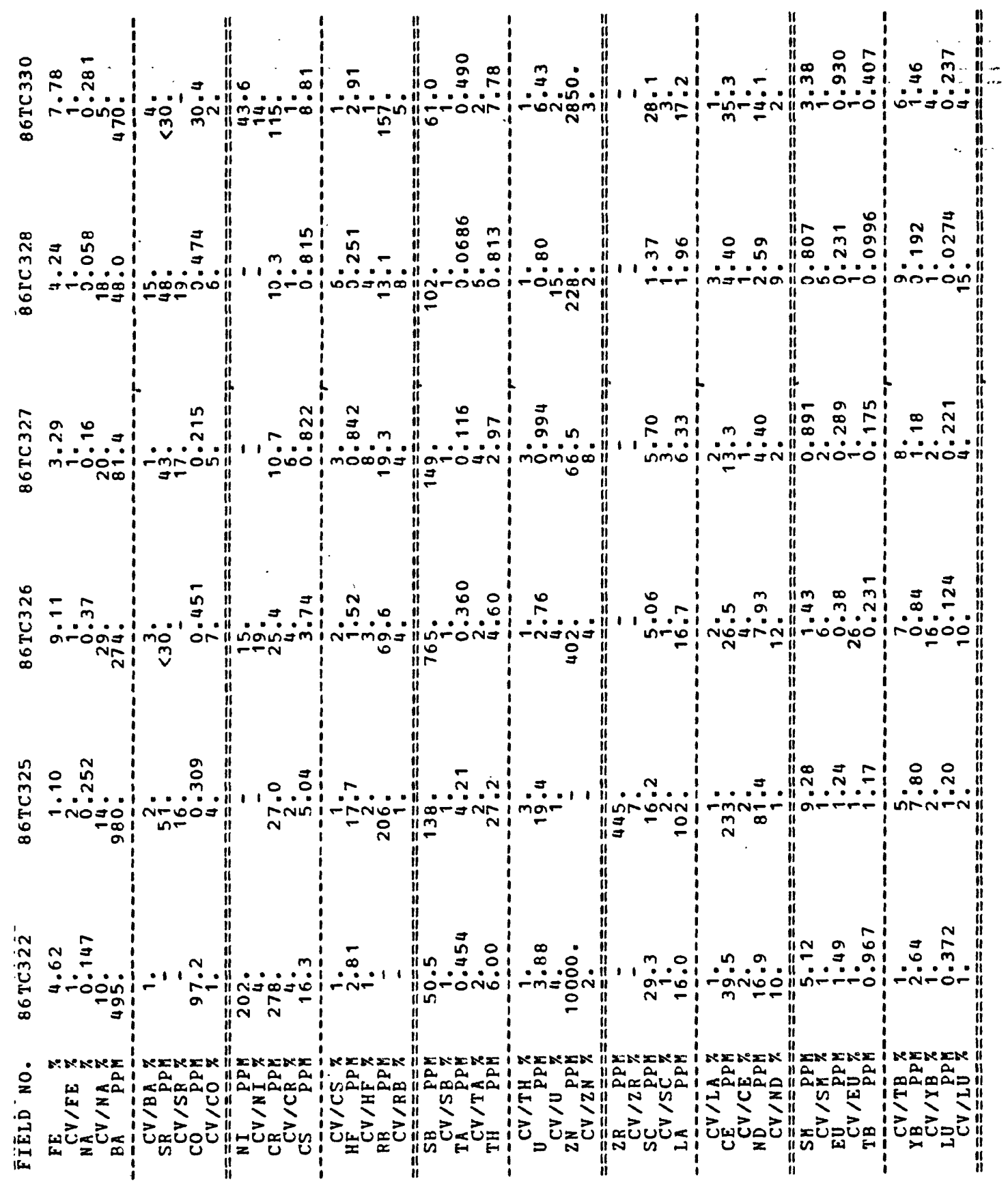




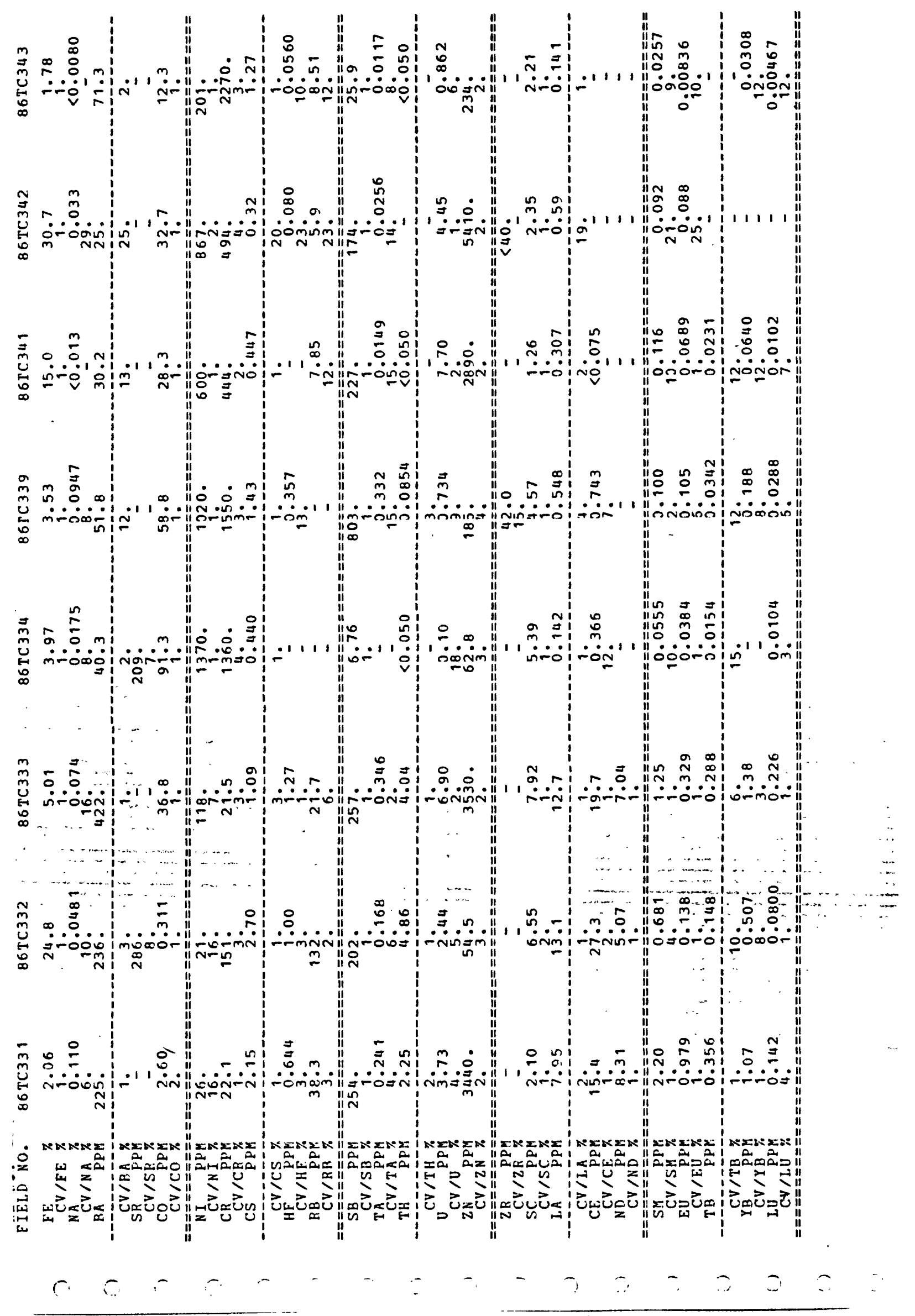




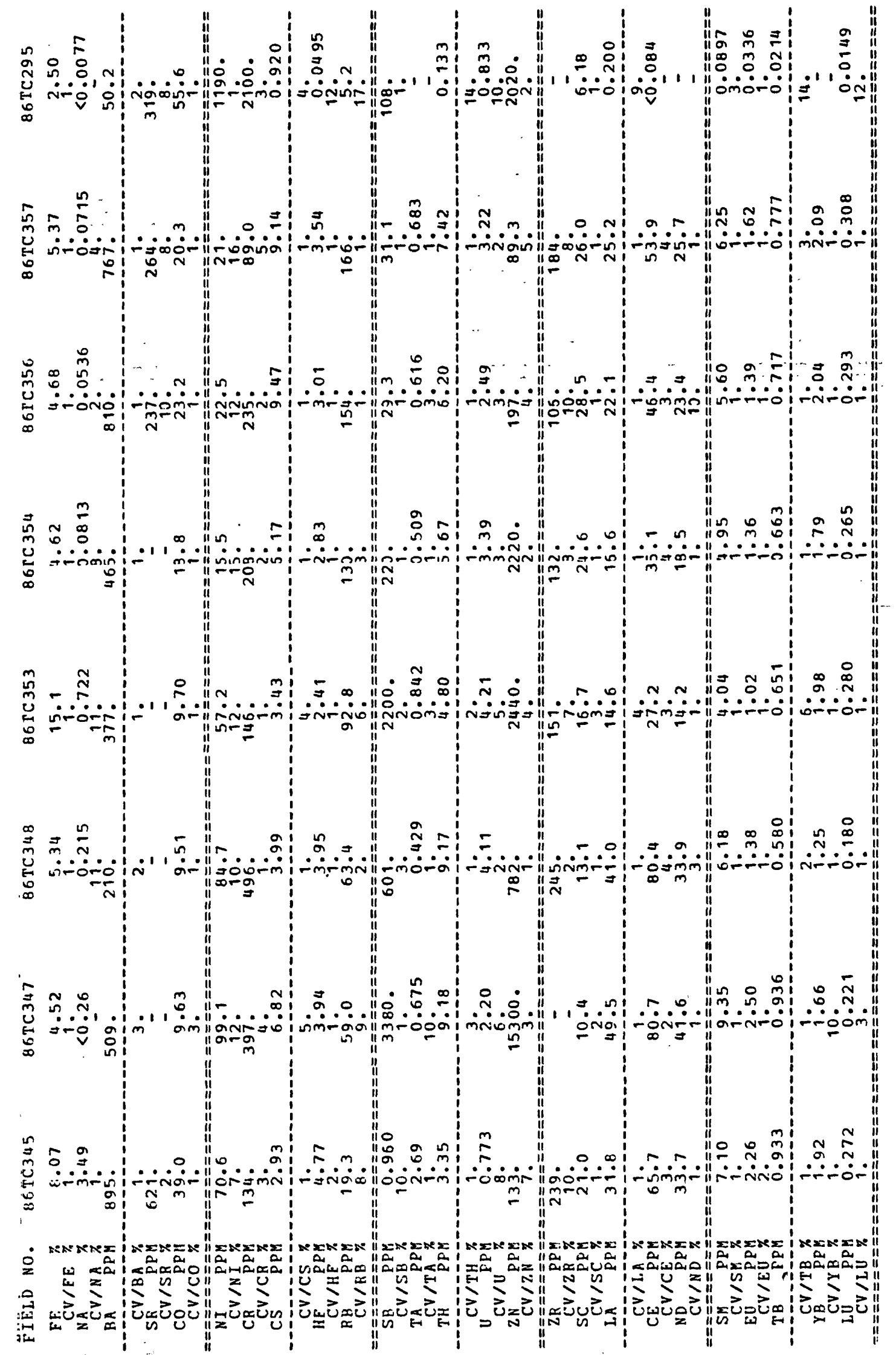




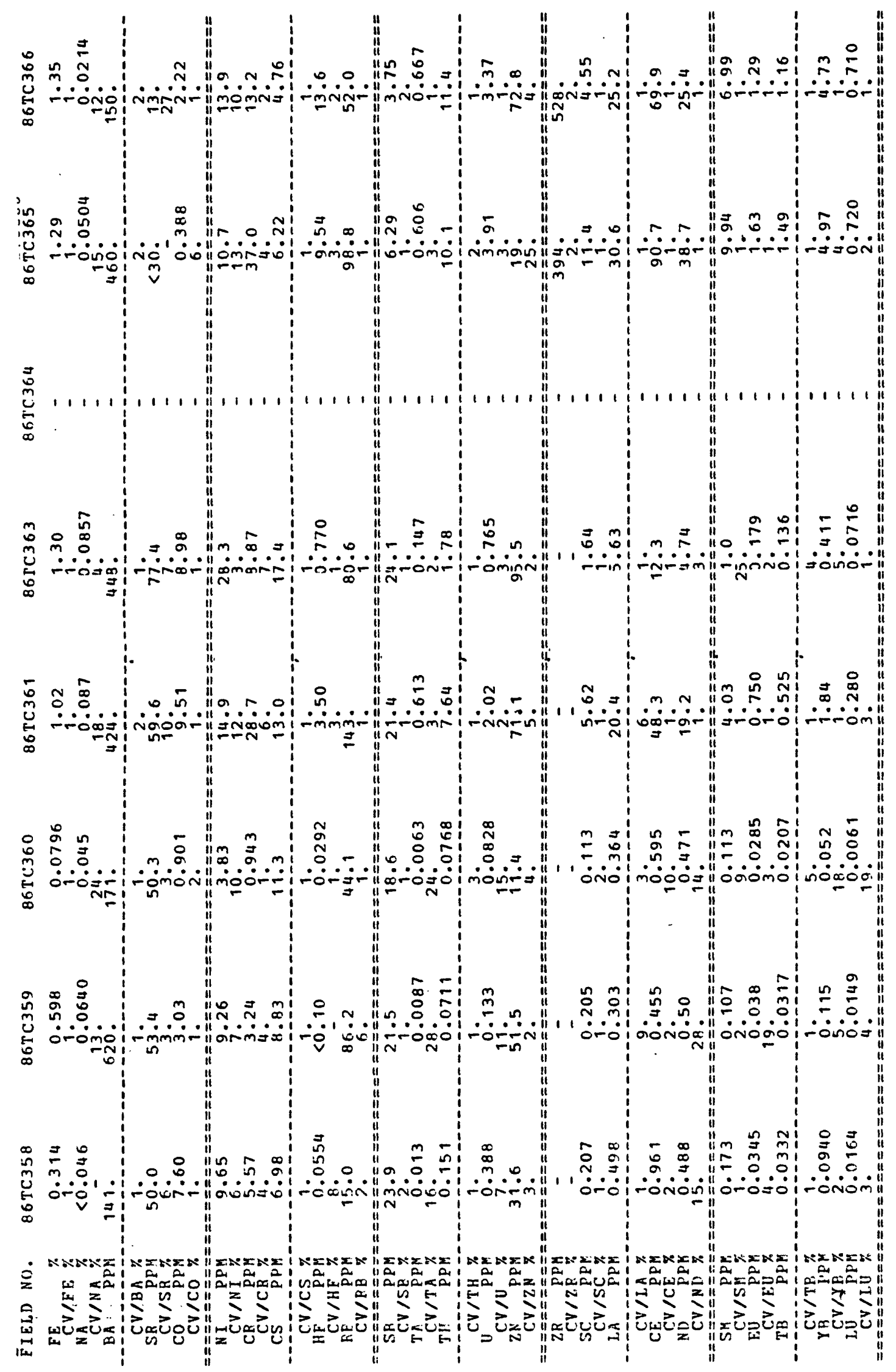




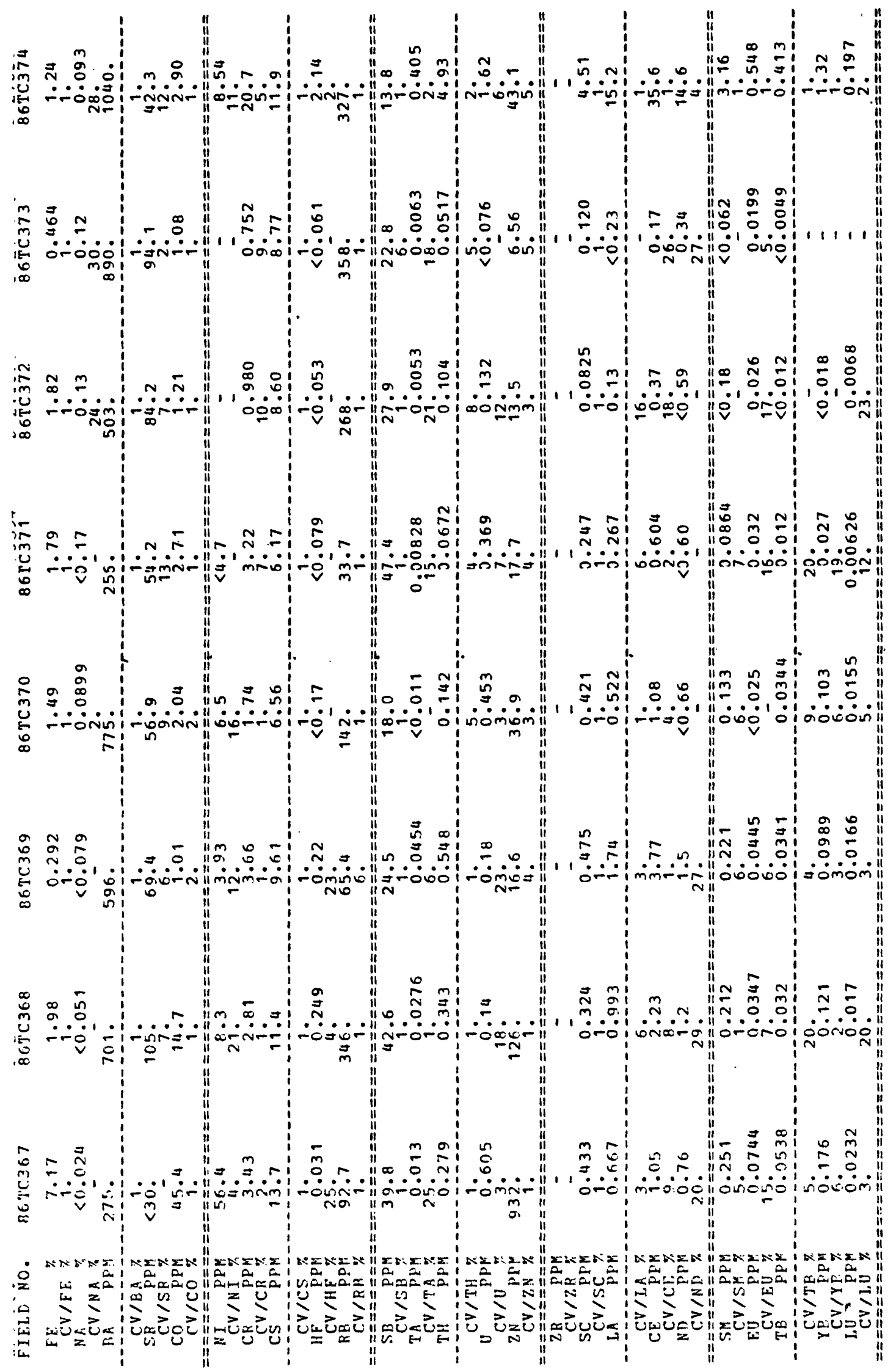




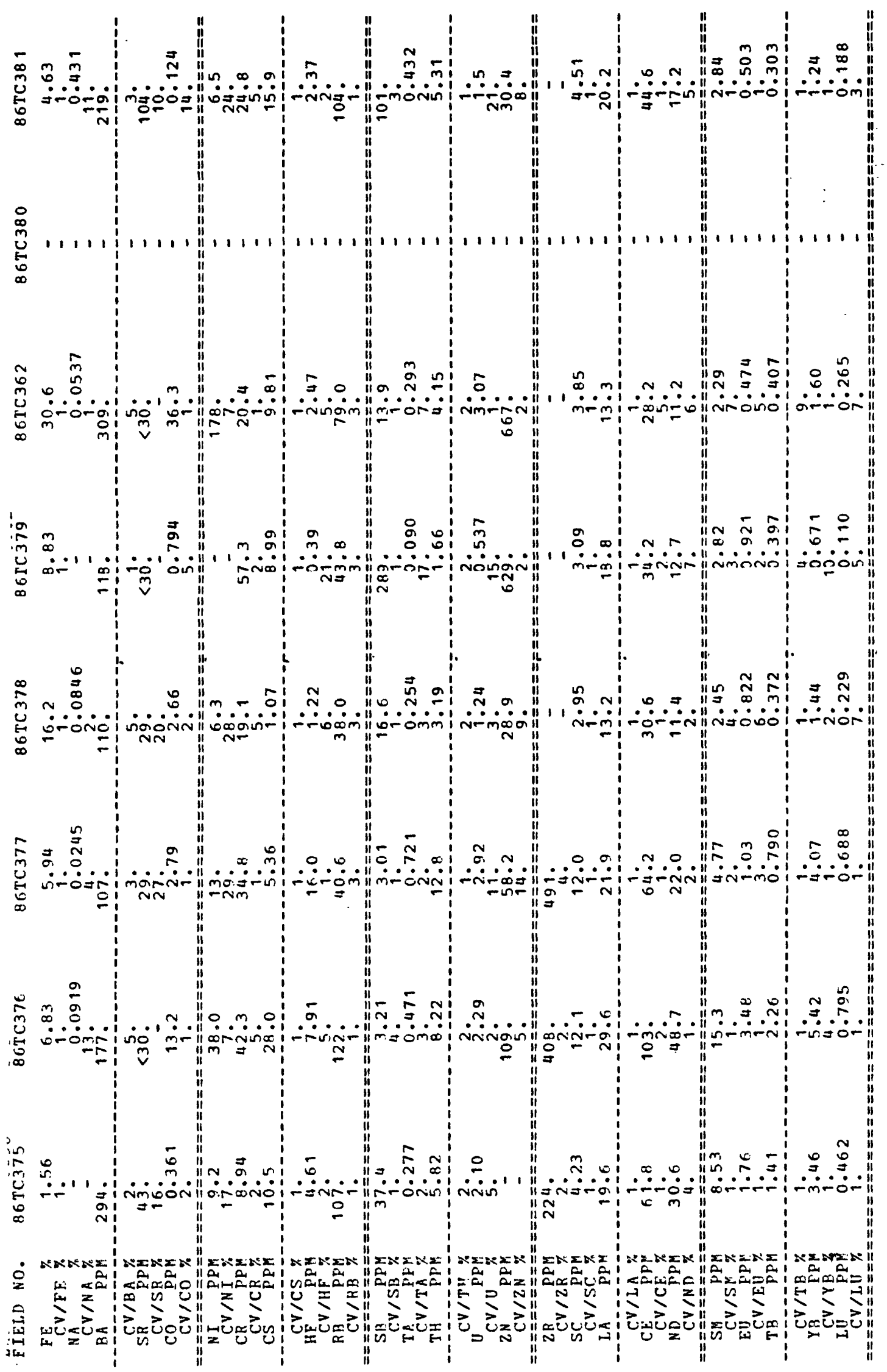




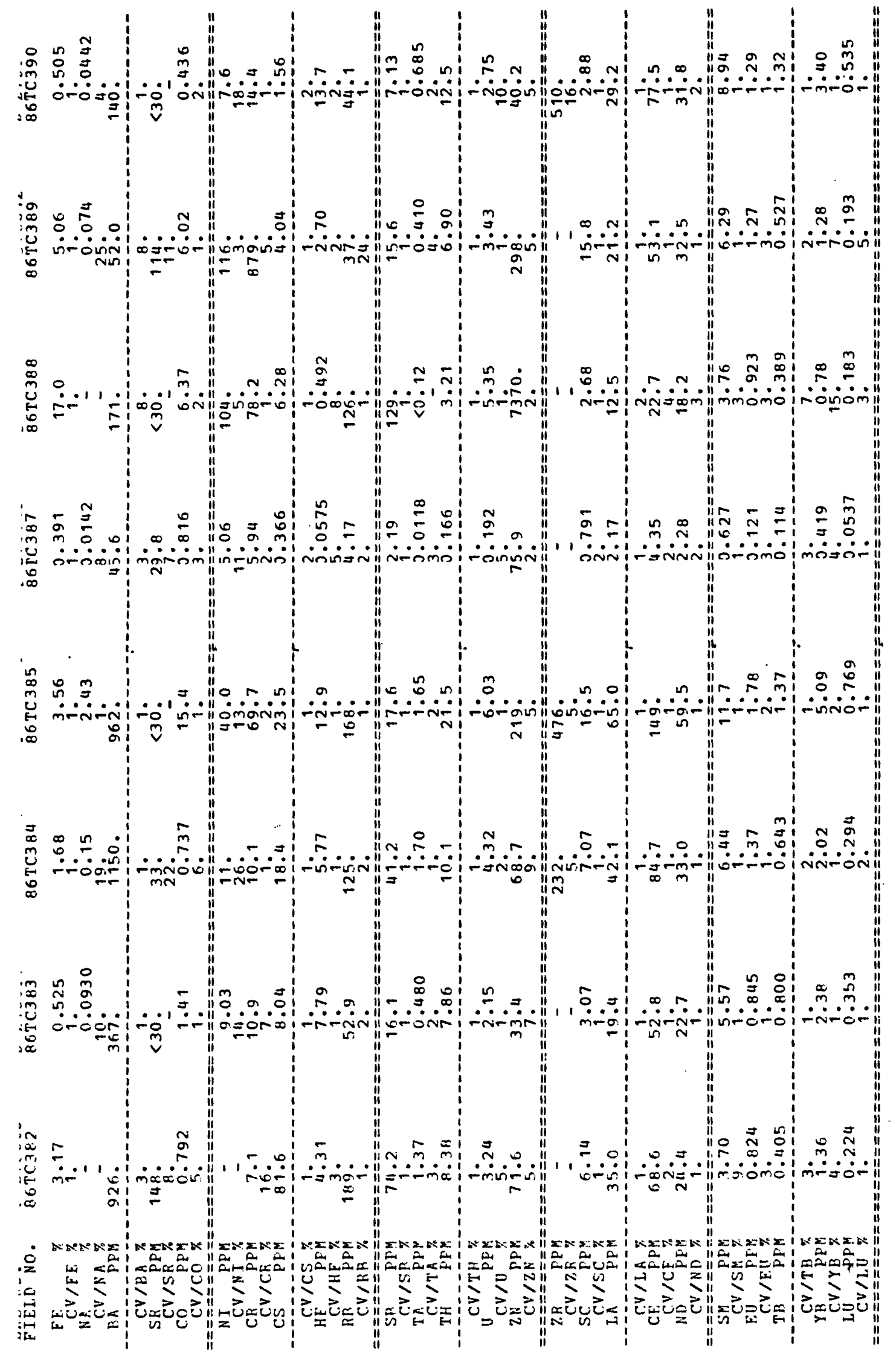




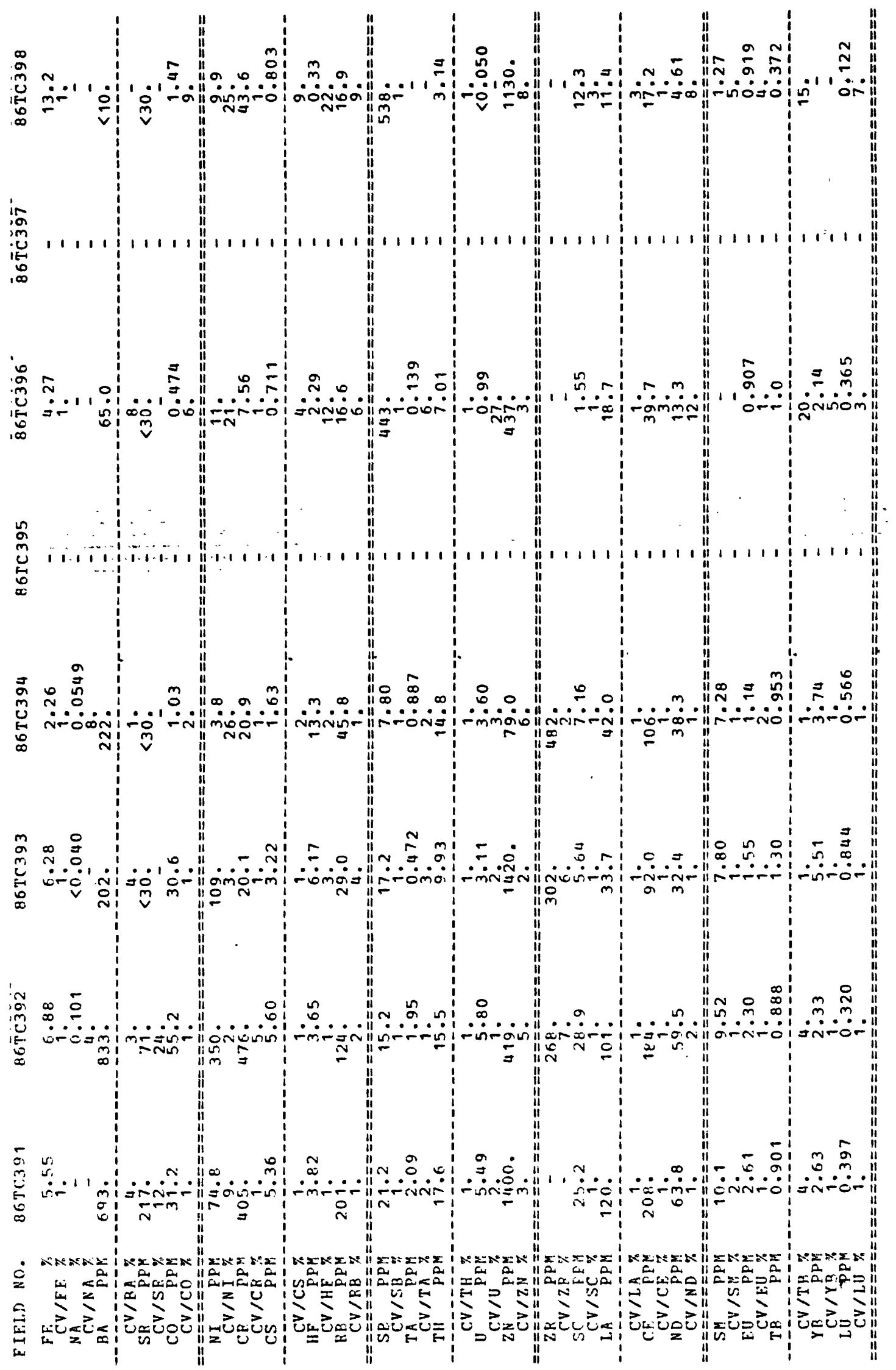




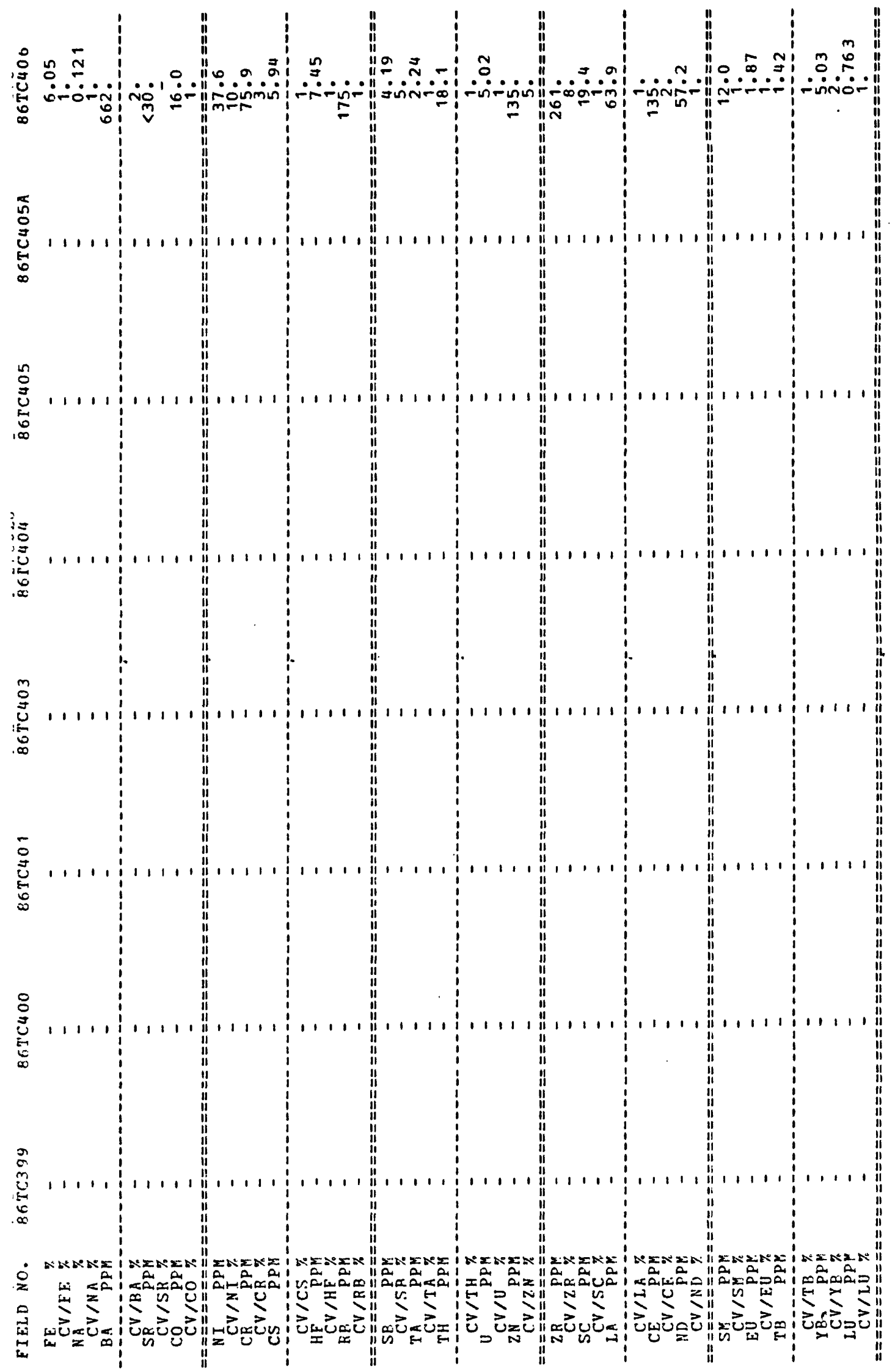




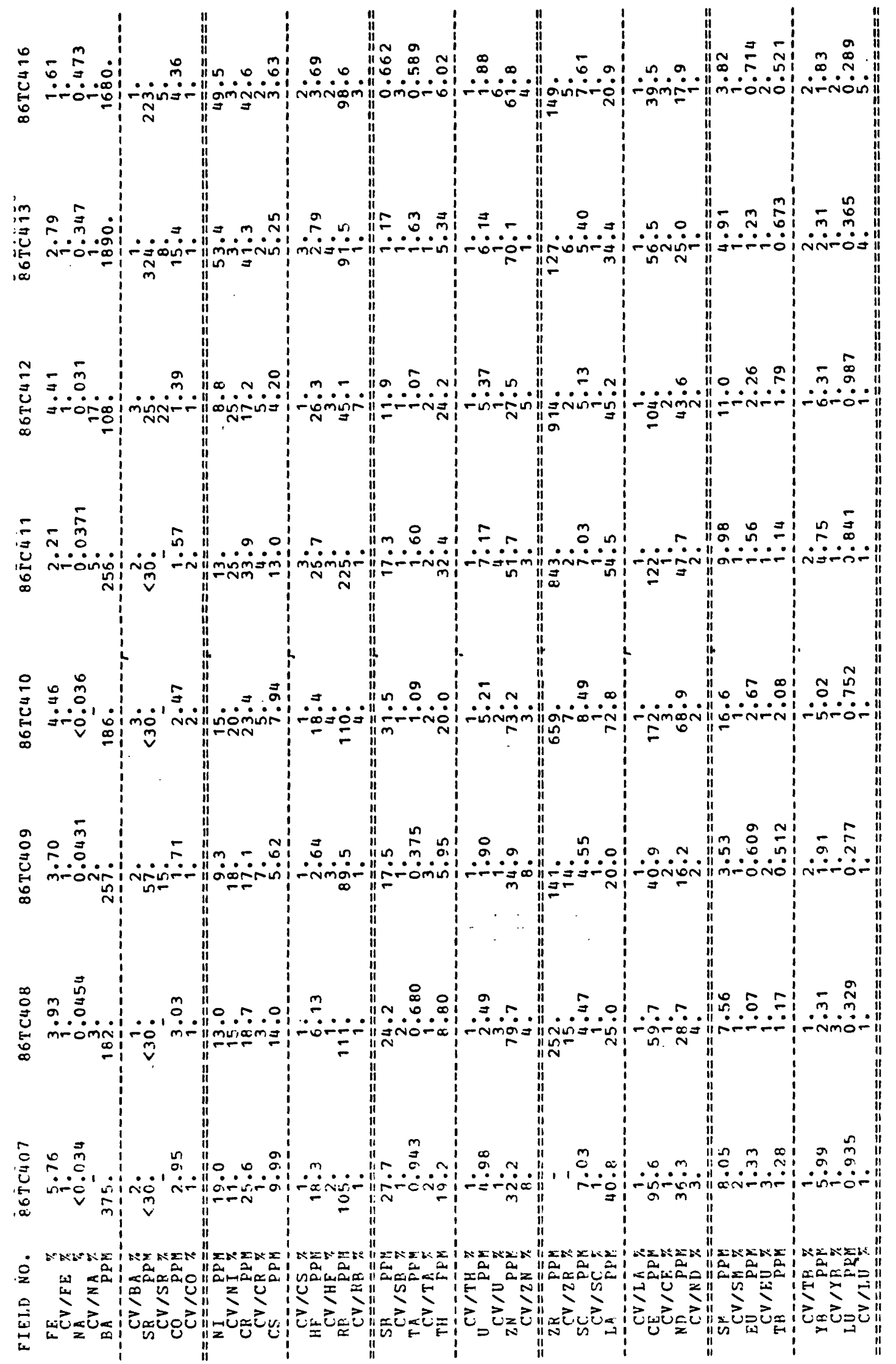




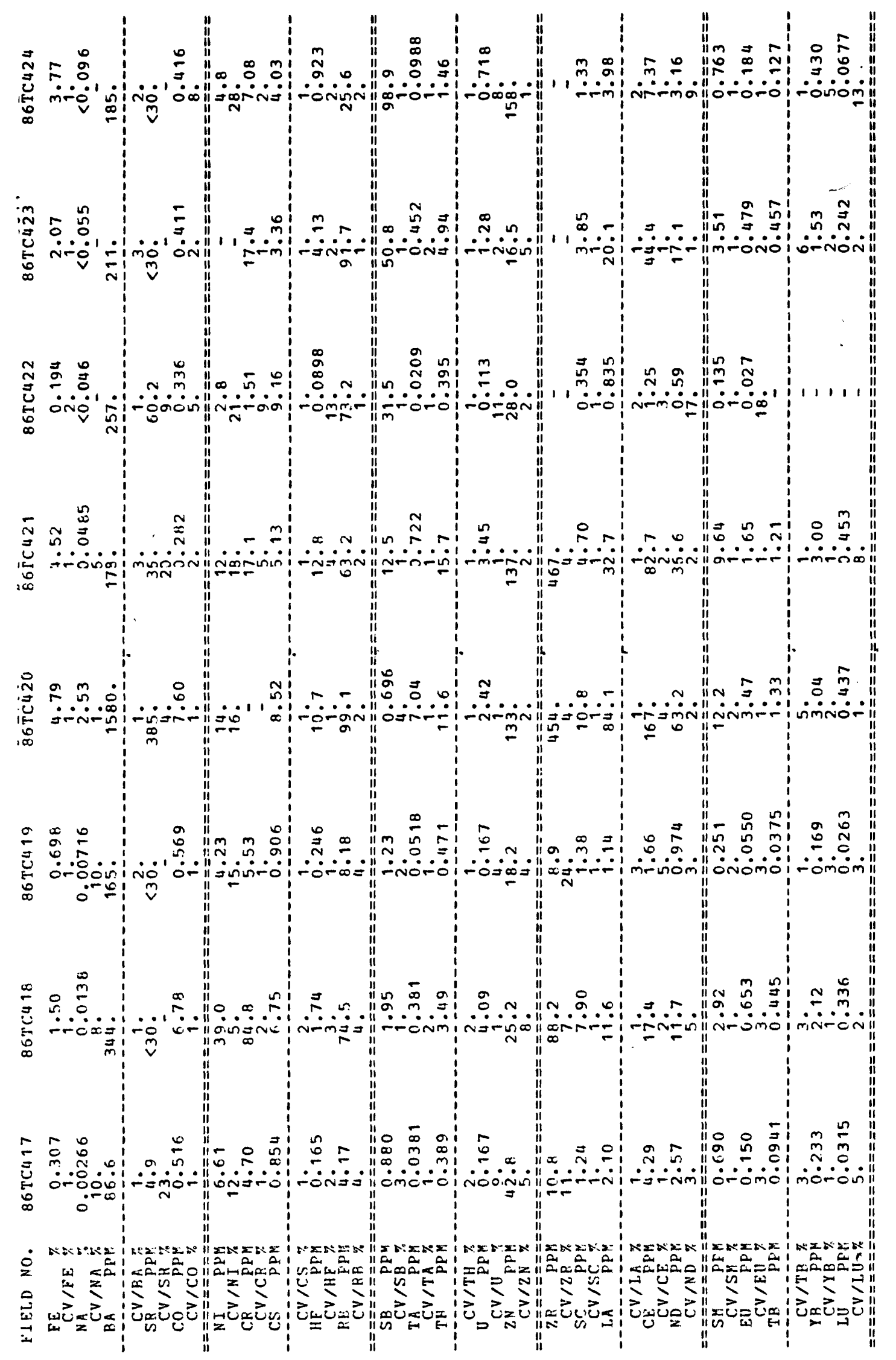




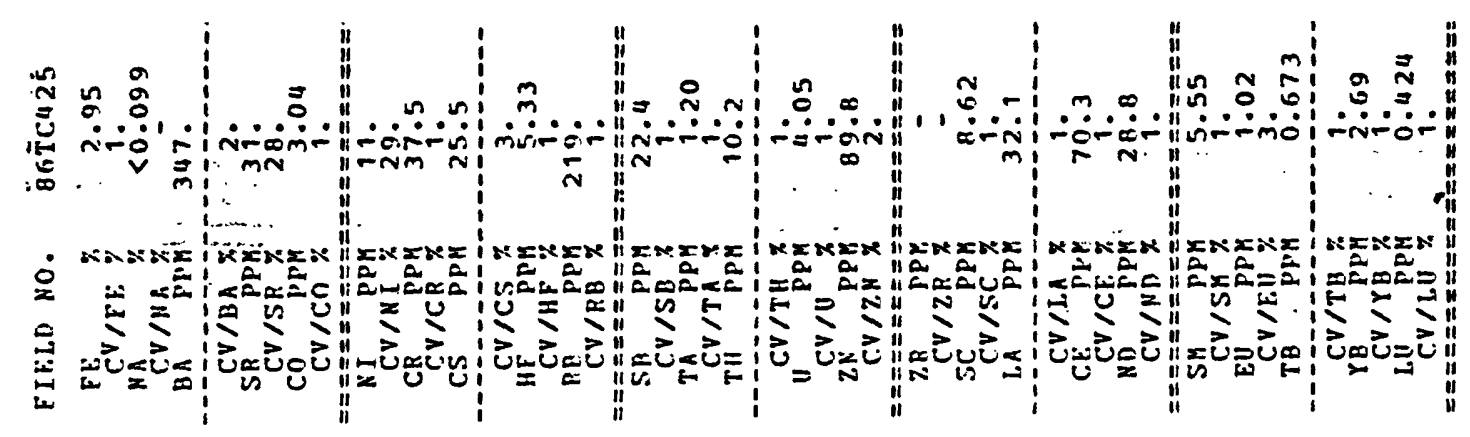

150 


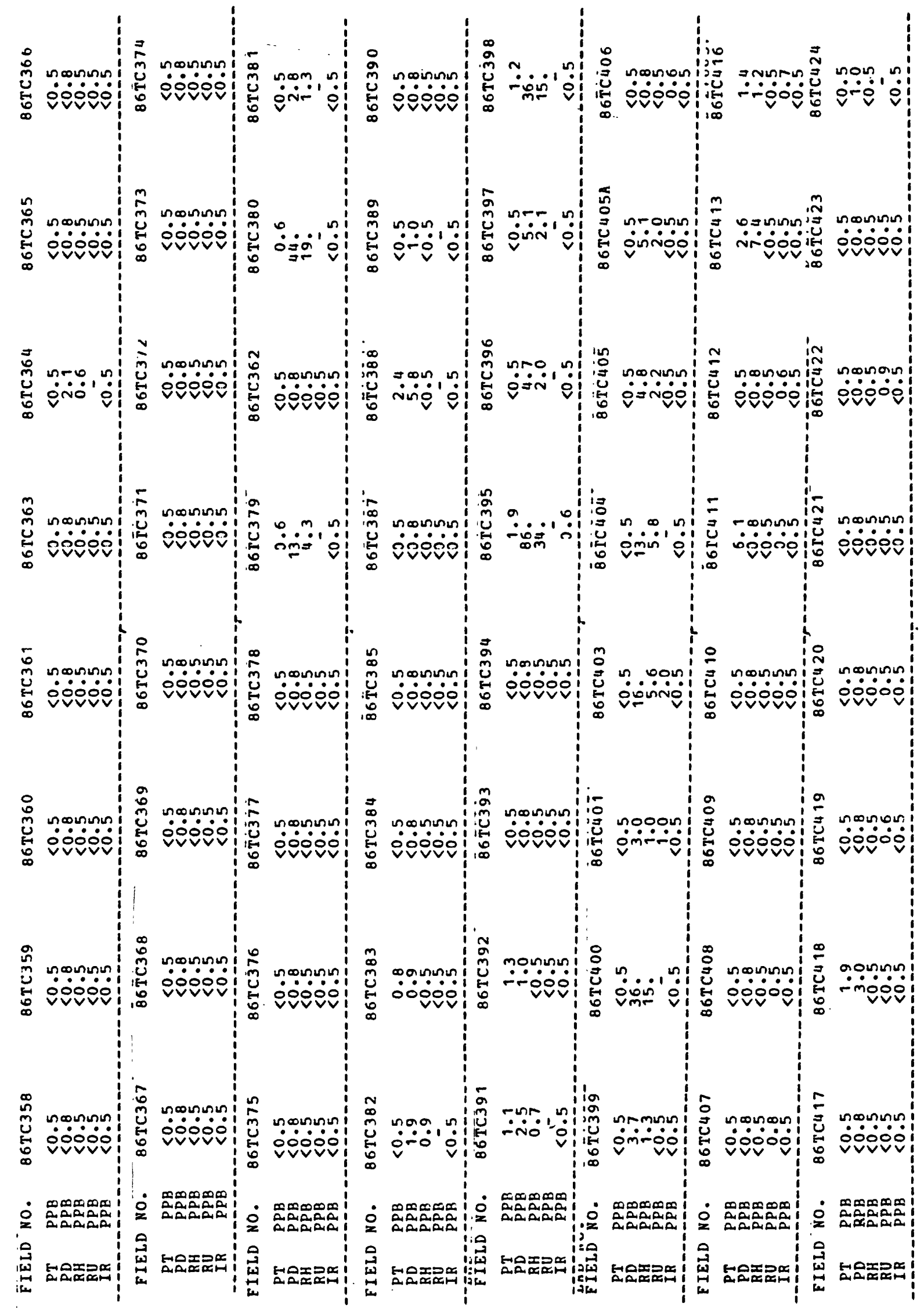




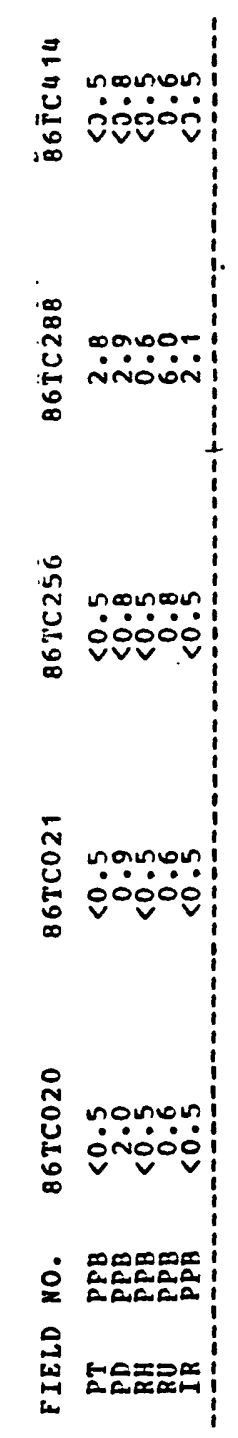

152 


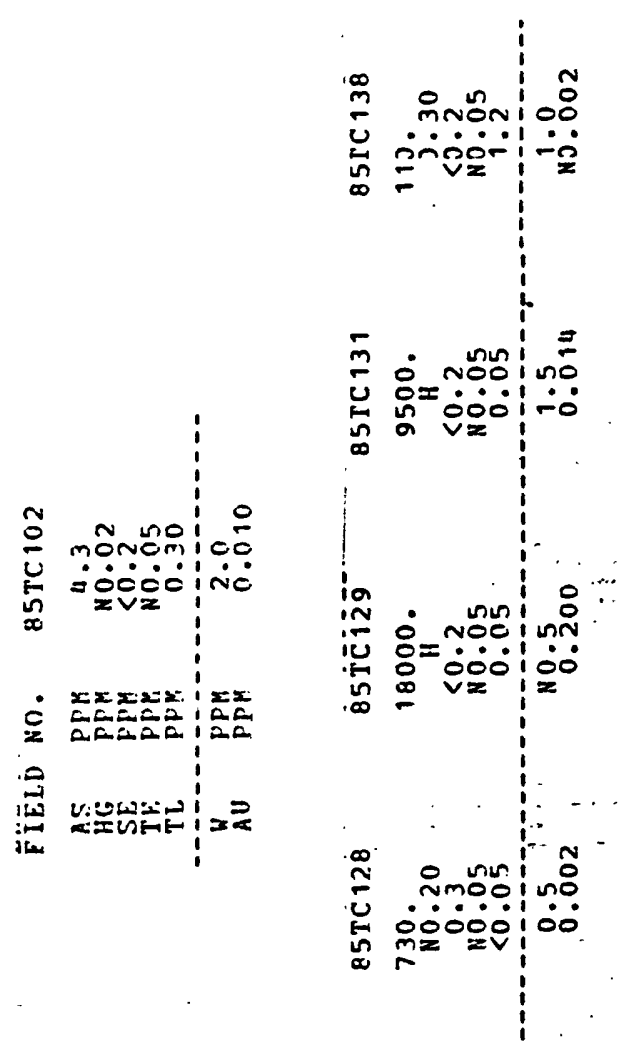




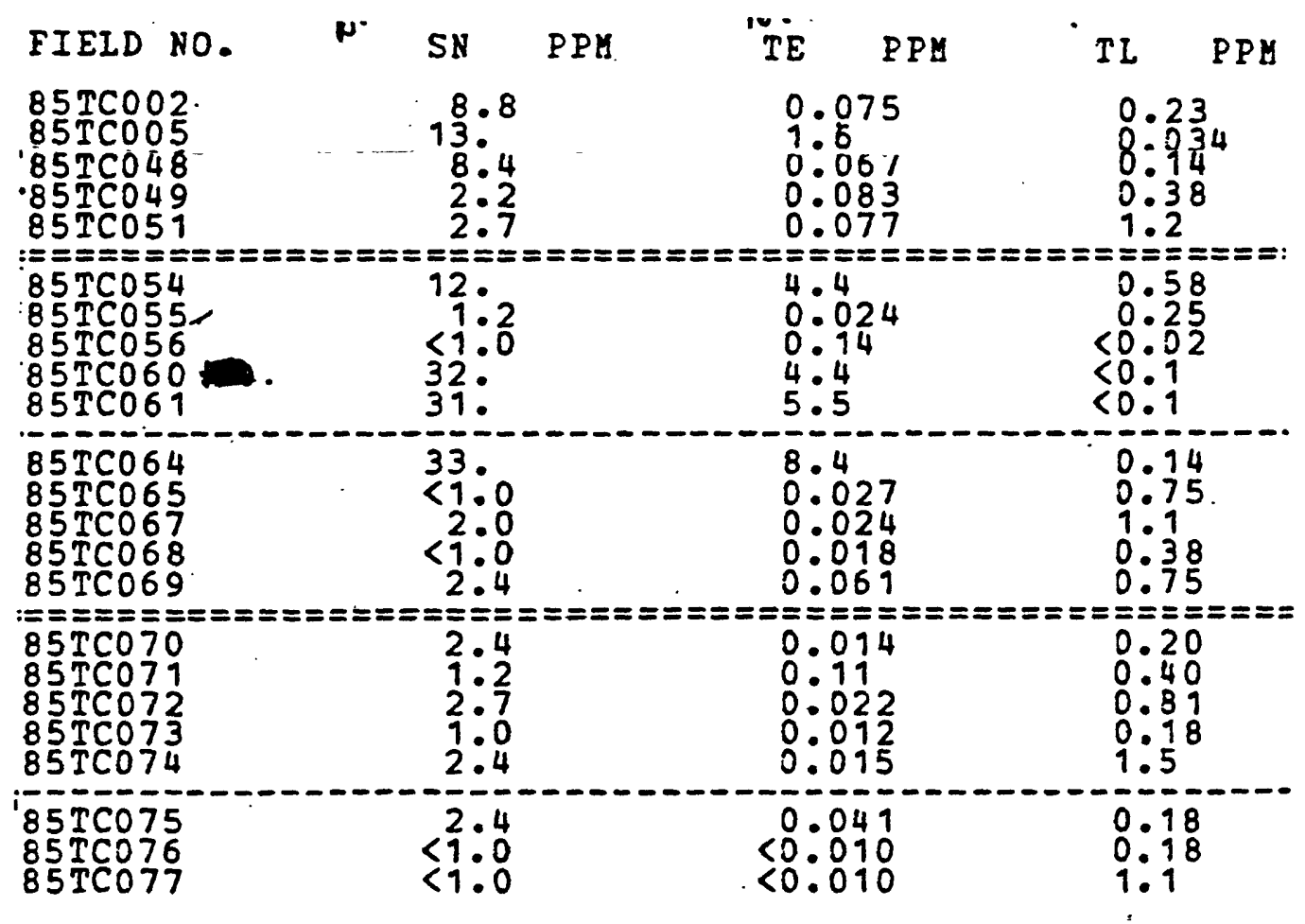




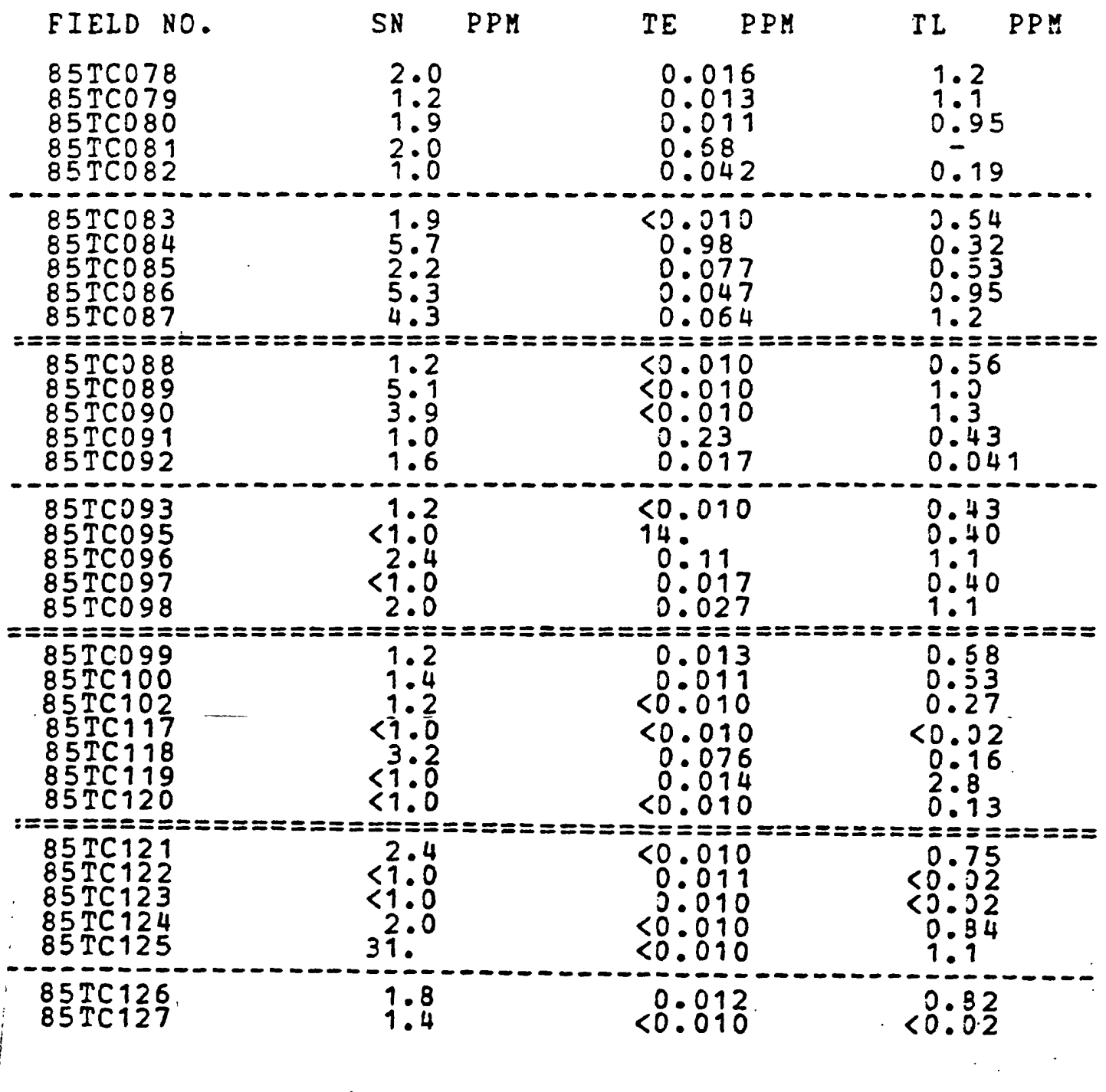

1 COYHENS

INTEREERENCE IN THE PBOCEDURE USED FOR DETEREINATIJN. 


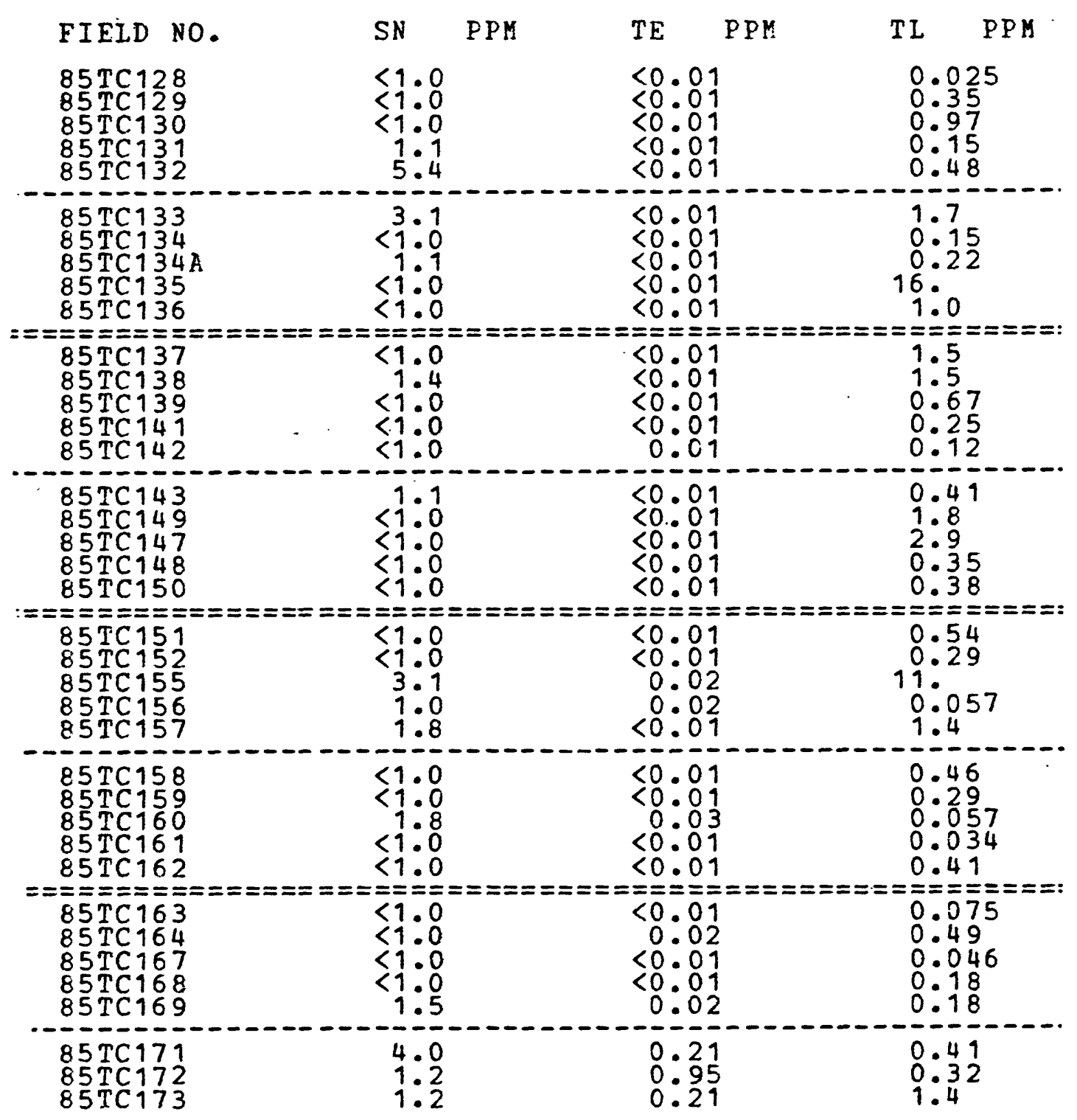

156 


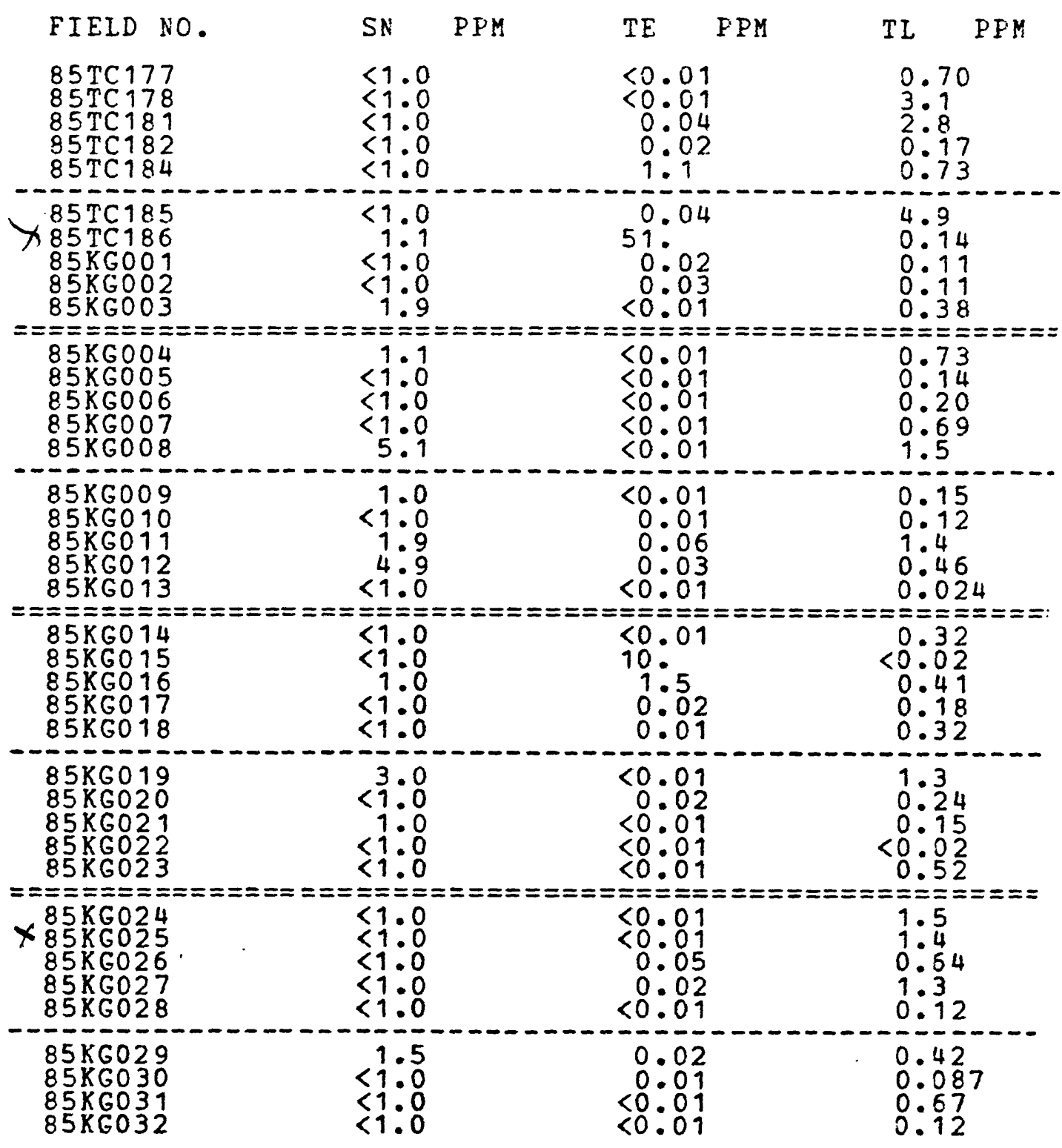




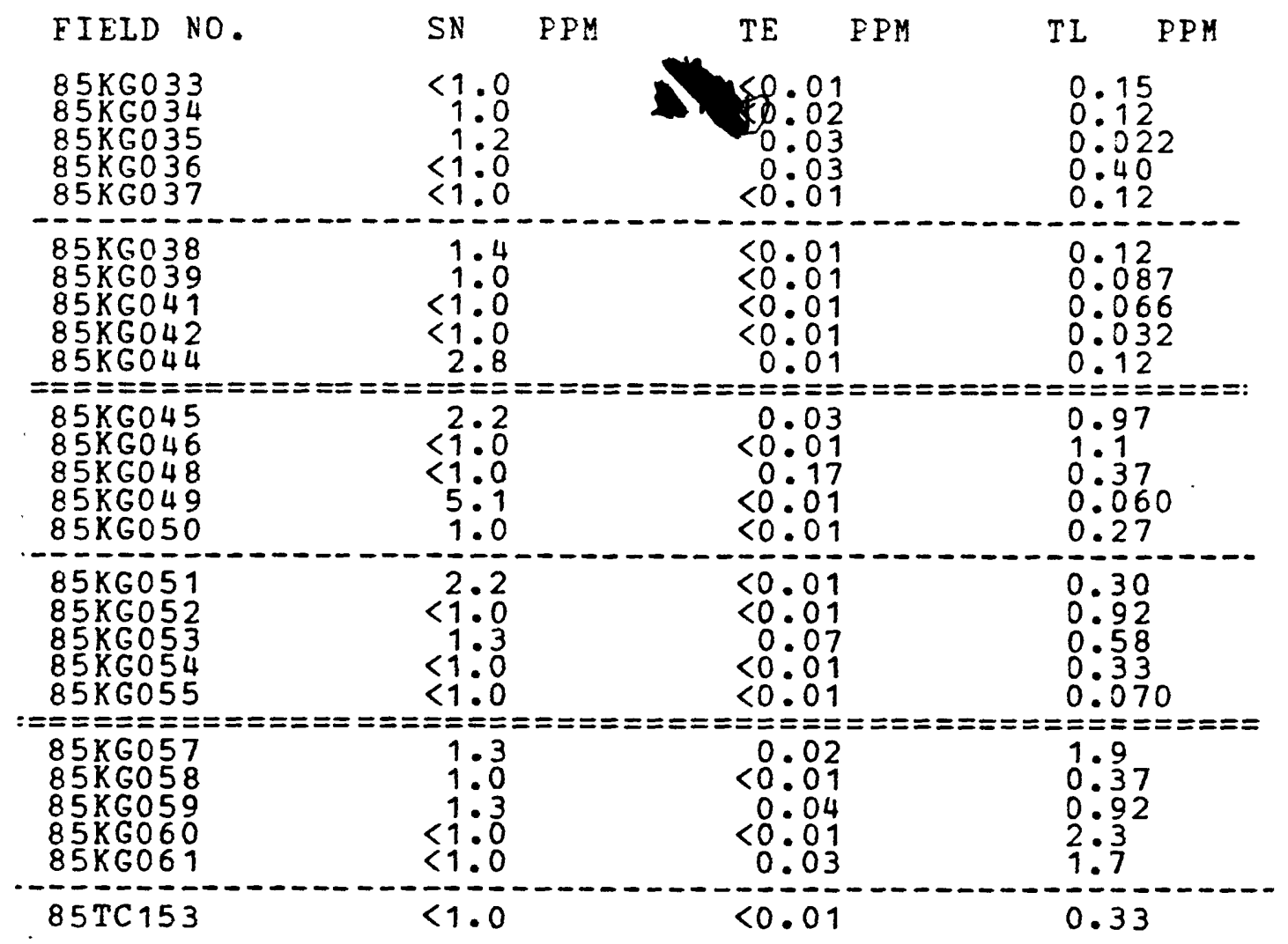




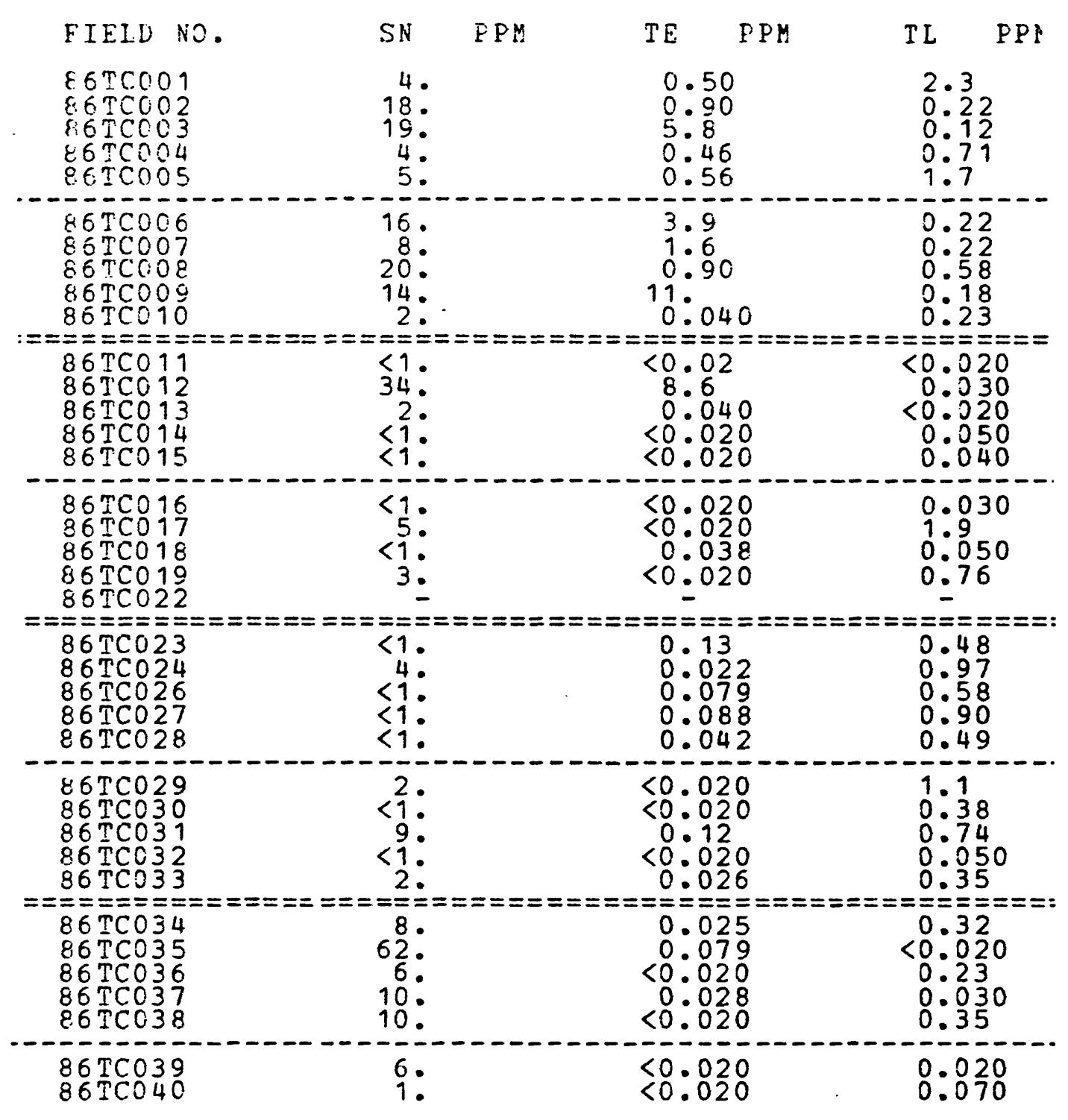

COMMENS D-275573 INSUFFICIENT SAMPLE TO COMPLETE ANALYSIS FOR SN,TE, AND
Sn done by XEF. 


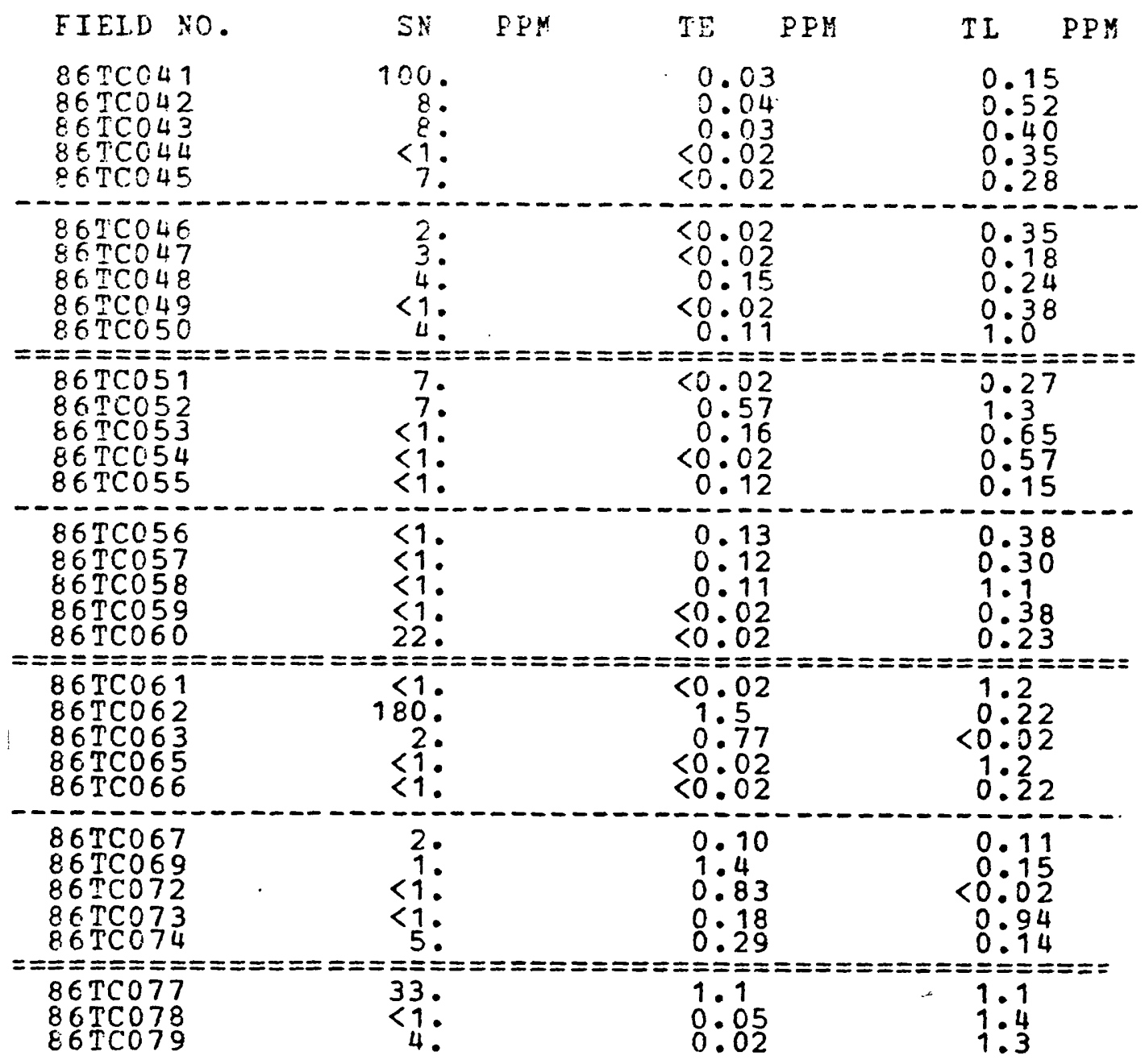

160 


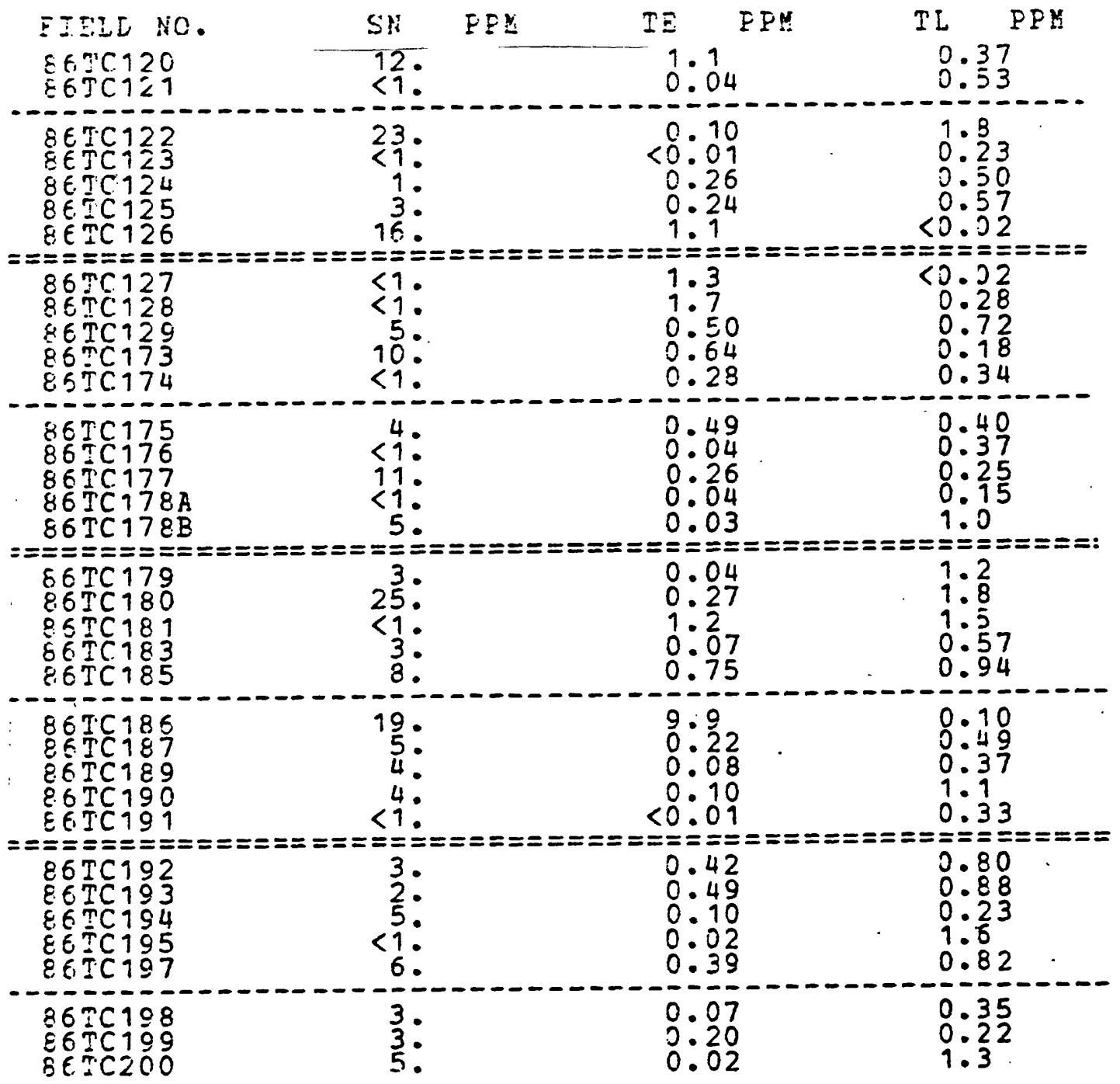




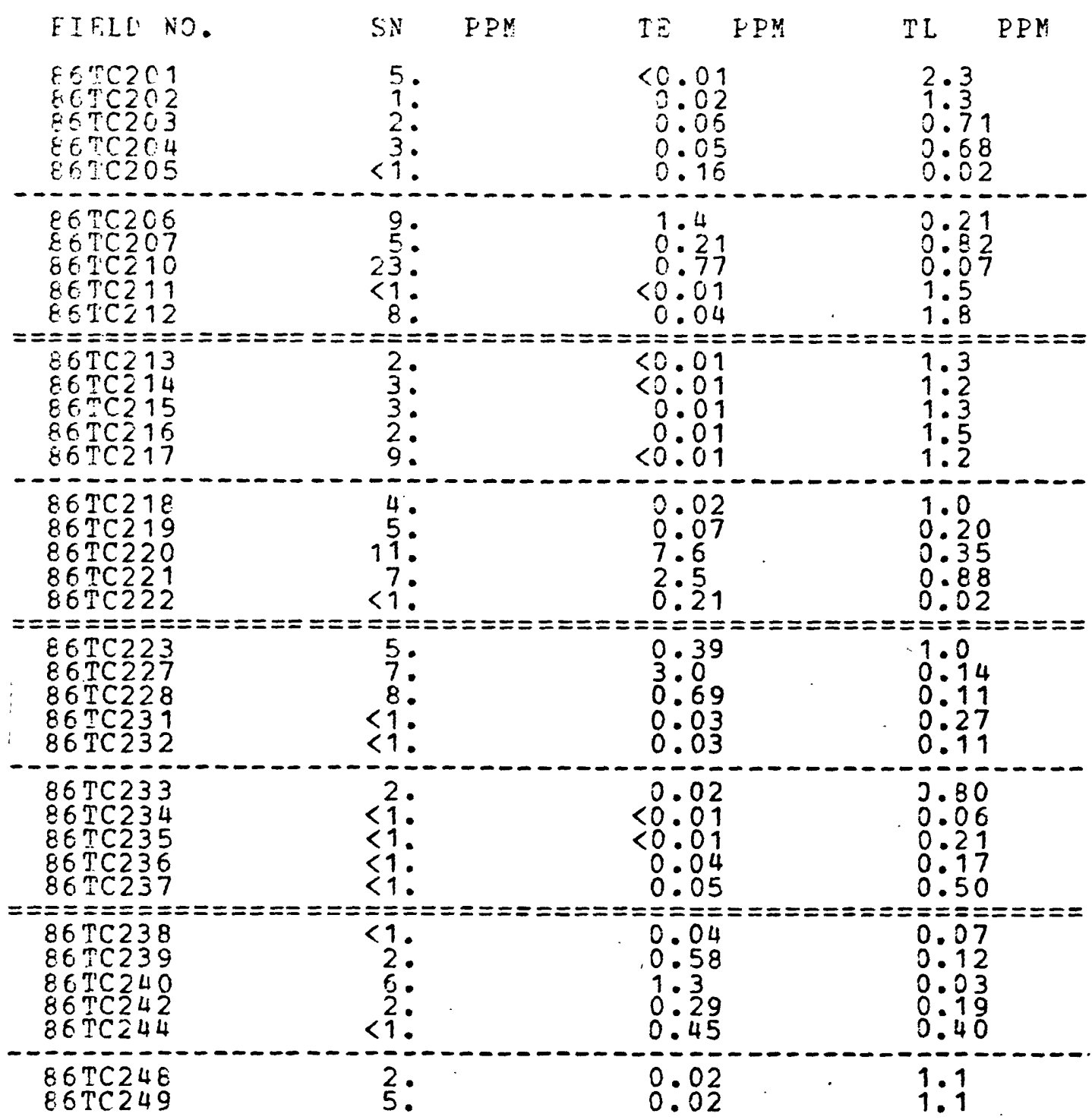




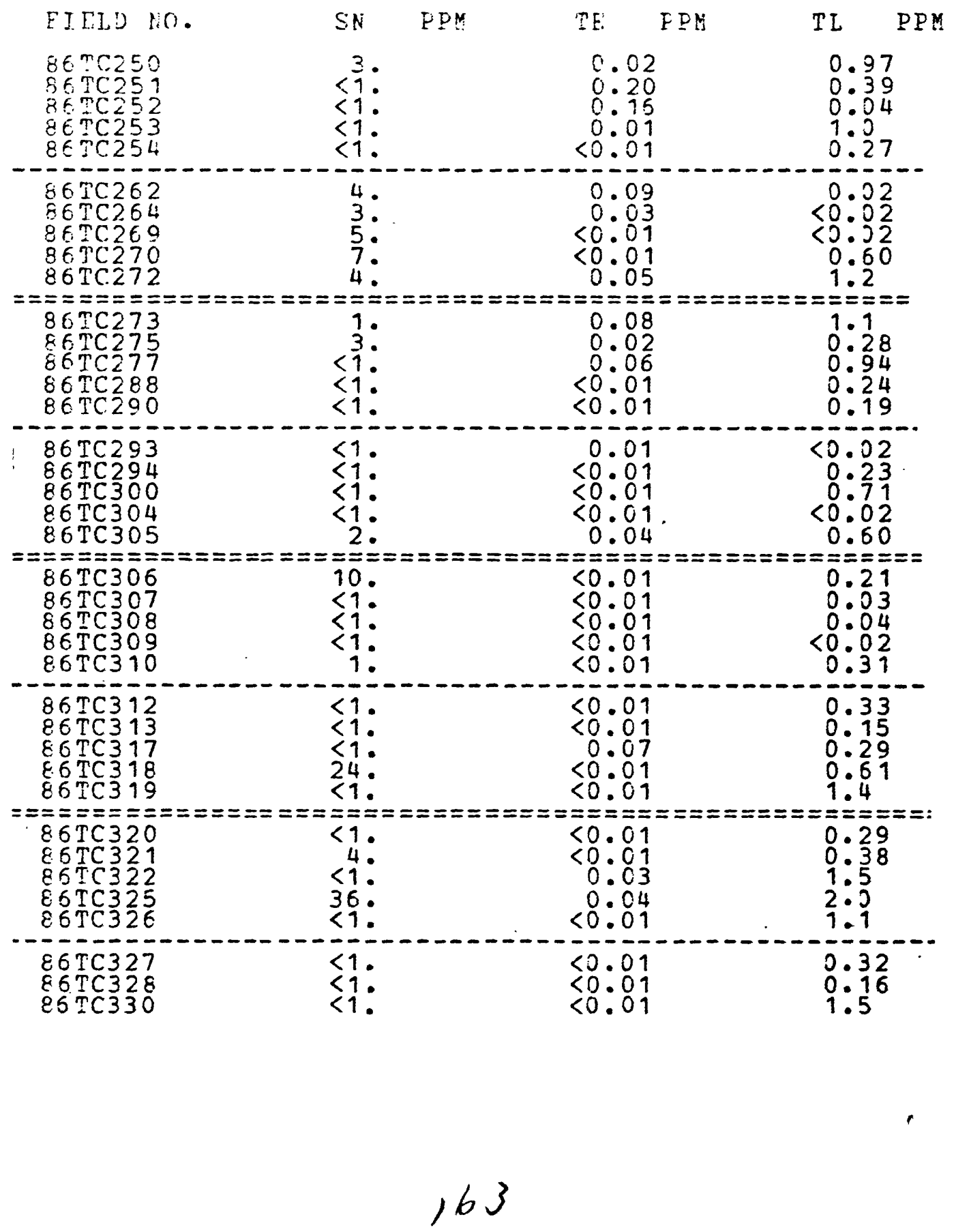




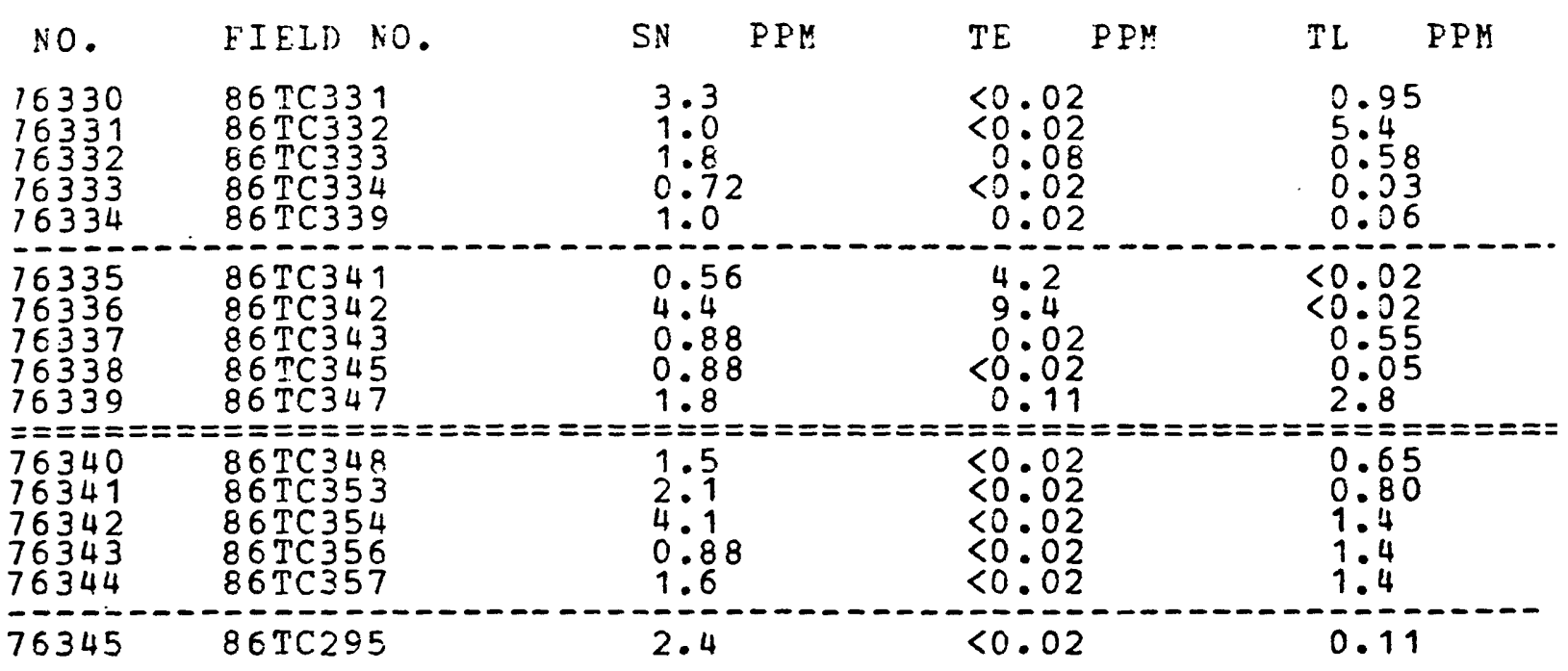




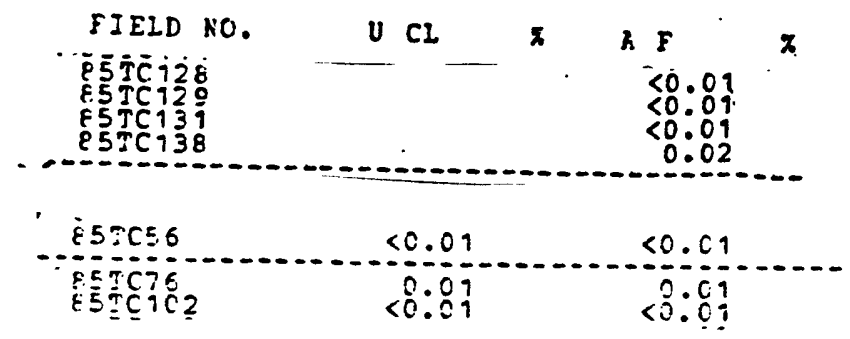

$\begin{array}{ll}\text { EIEID NO. } & \text { F } \\ 85 T C 002 & 0.07 \\ 85 T C 005 & 0.14 \\ 85 T C 048 & 0.10 \\ 85 T C 049 & 0.04 \\ 85 T C 057 & 0.19\end{array}$

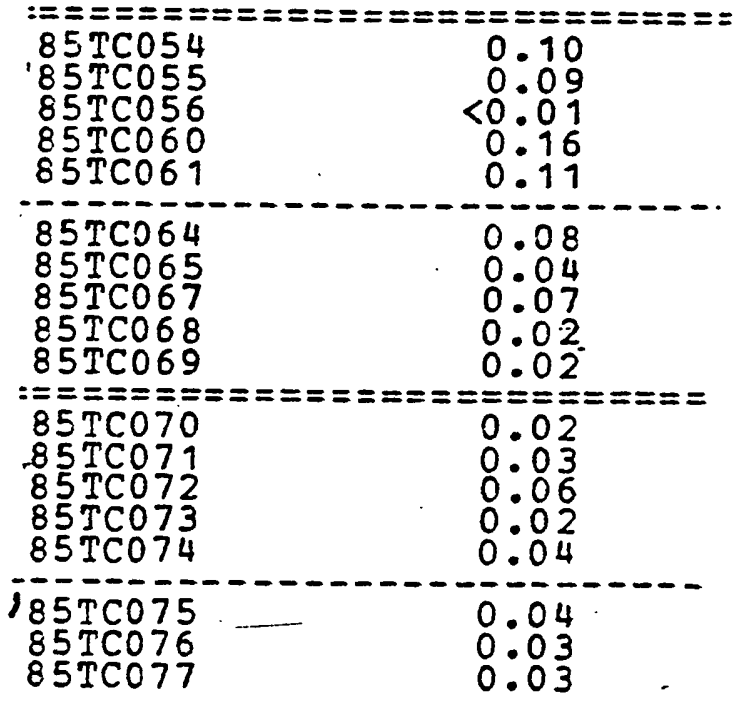

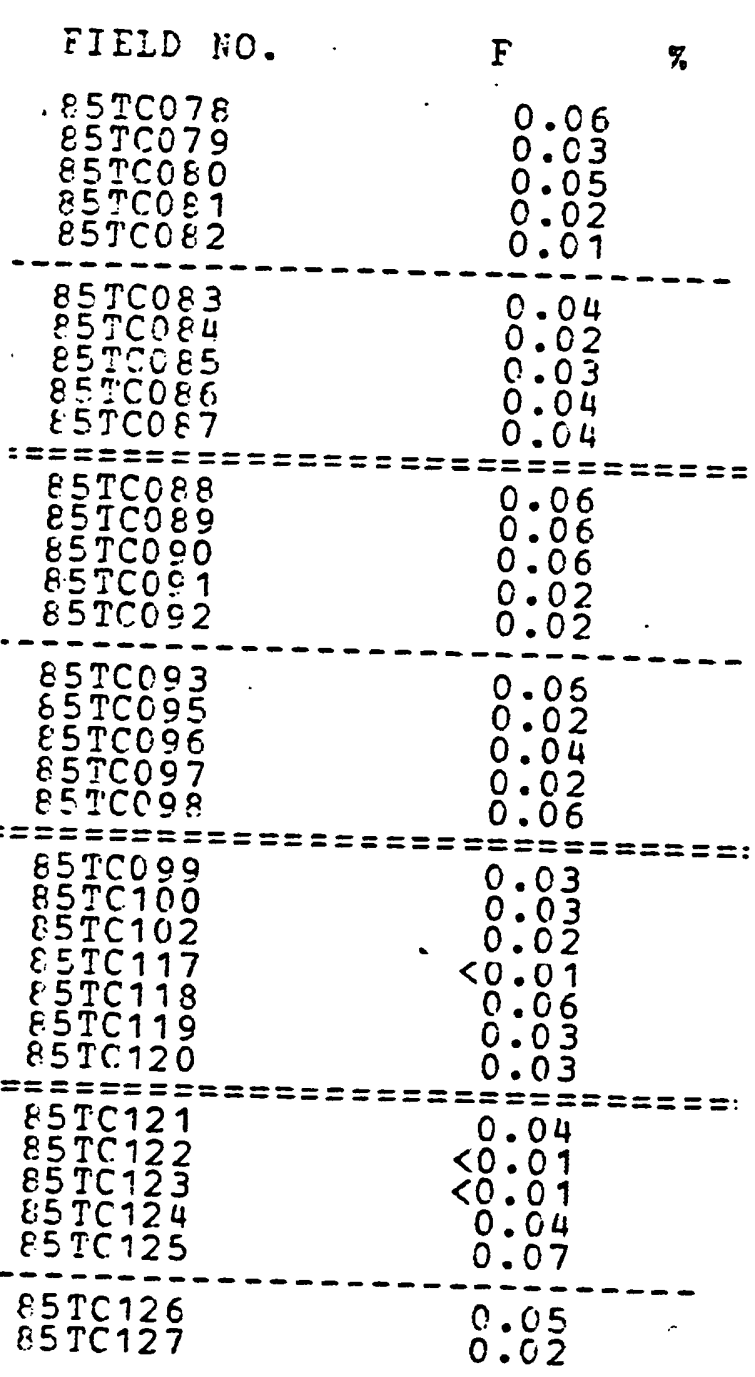


FIEID NO.

FIEID NO. F \%

$85 \mathrm{TC} 177$

g5TC128 $<0.01$

$\begin{array}{ll}85 \mathrm{~T} C 128 & 0.01 \\ 85 \mathrm{TC} 29 & 0.02 \\ 85 \mathrm{TC} 130 & 0.01\end{array}$

$85 \mathrm{TC} 131$

0.02

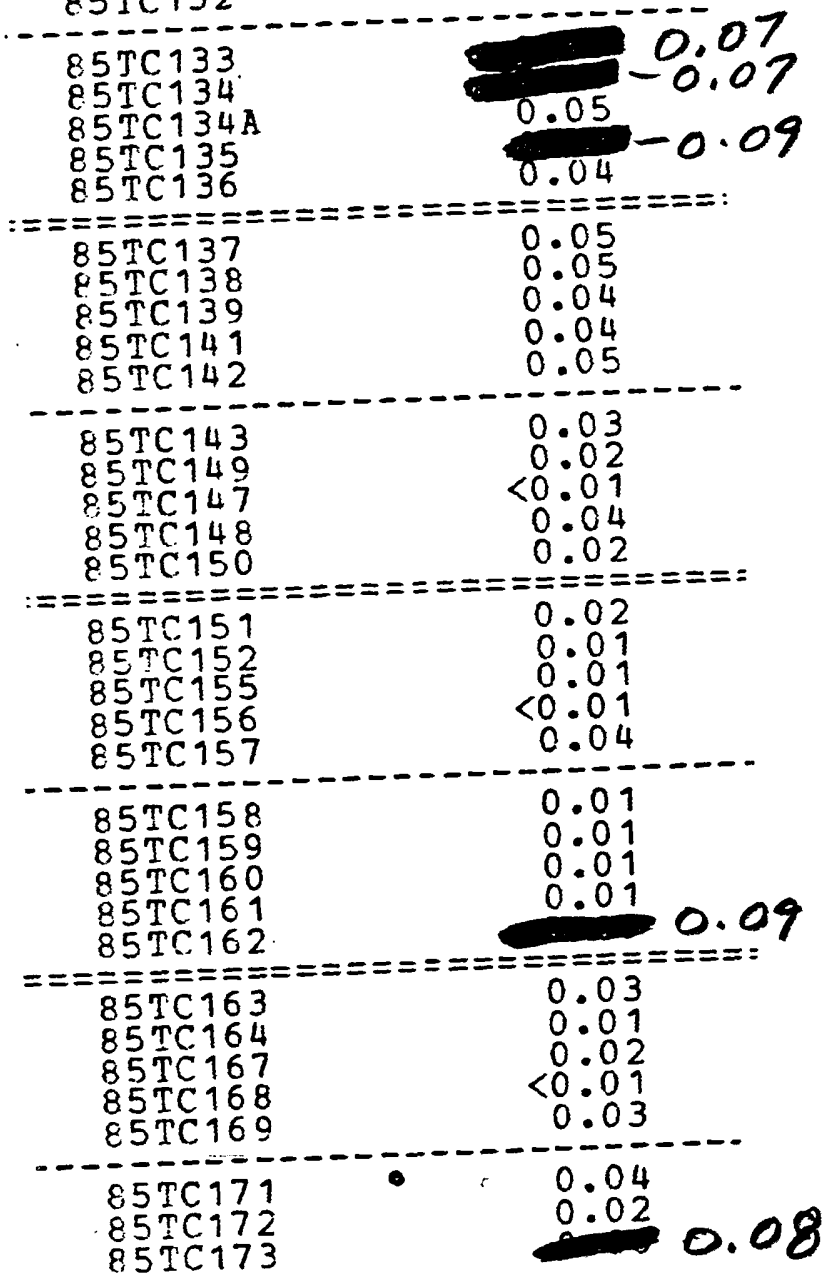

$85 \mathrm{~T} C 178$

$85 \mathrm{TC} 181$

85 TC 182

$85 \mathrm{TC} 184$

$<0.01$
0.01

$-1----1$

$<0.01$

$85 \mathrm{TC} 185$

Q5TC 98.6

E5KG001

$85 K G 002$

$85 \mathrm{KG003}$

$<.01$
0.02

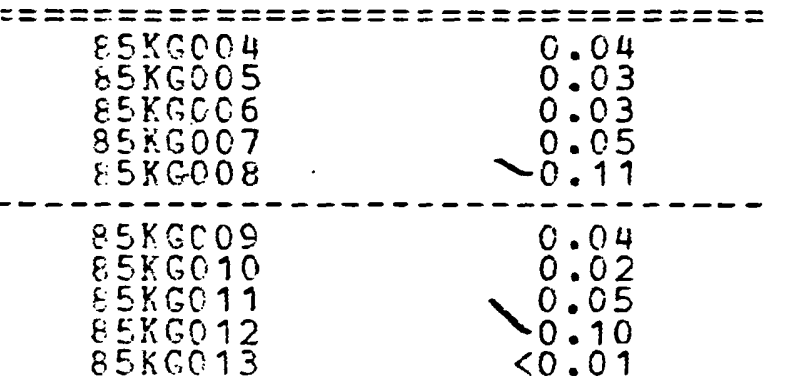

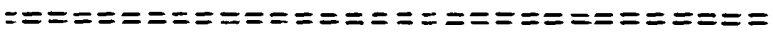

$85 K 6014$

8536095

$85 K G 016$

$85 \mathrm{KCO} 17$

0.03

$85 \mathrm{KGO} 18$

0.02

$85 \mathrm{KGO} 19$

0.01

$85 \mathrm{KG020}$

$85 K G 021$

$85 \mathrm{k} 6022$

0.01

85 KGO23

0.06

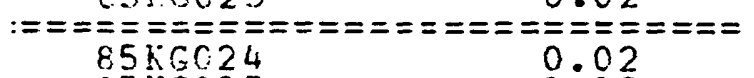

$85 K G 025$

$85 \times 6026$

$85 \mathrm{KGO} 27$

0.01

$85 \mathrm{KGO} 28$

$<0.01$

0.02

$85 \mathrm{KGC29}$

$85 \mathrm{~K} 6030$

$85 K 6031$

$85 K 6032$

$85 \mathrm{KGO33}$

$85 \mathrm{KCO} 34$

$85 \mathrm{KCO} 35$

$8.5 \mathrm{KGO} 36$

$85 \mathrm{KGO} 37$

0.02

0.03

0.05

0.03

$\begin{array}{ll}85 \times G 038 & 0.01 \\ 85 \times 6039 & 0.01 \\ 85 K 6041 & 0.02 \\ 85 K G 042 & 0.03 \\ 85 K_{0044} & 0.03\end{array}$

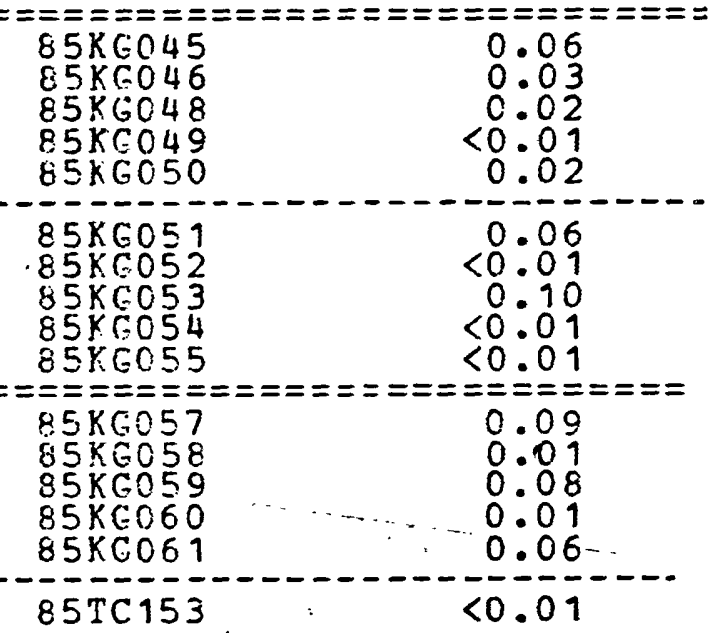




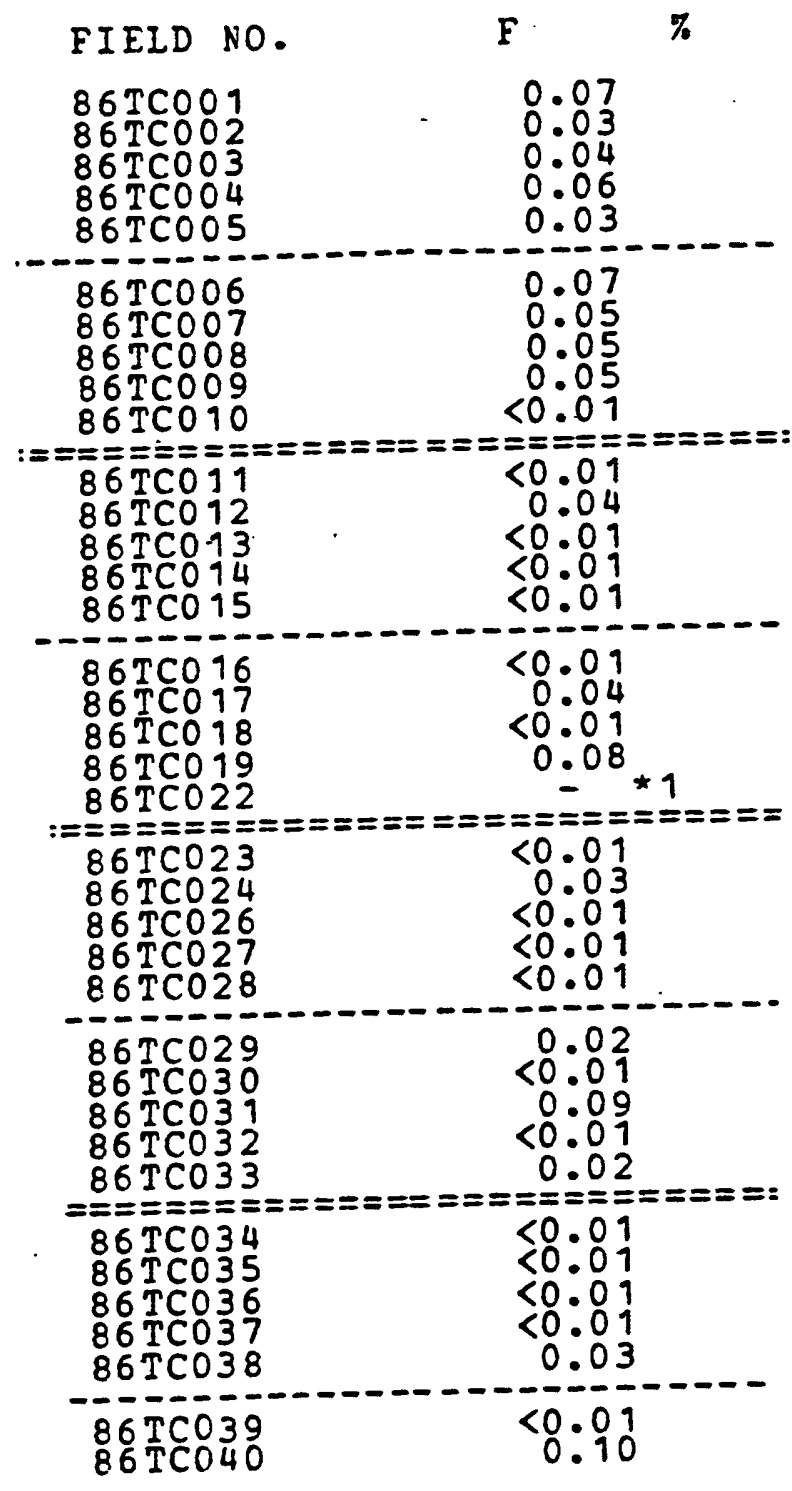

ニニニニニニニニニ

NOTES :

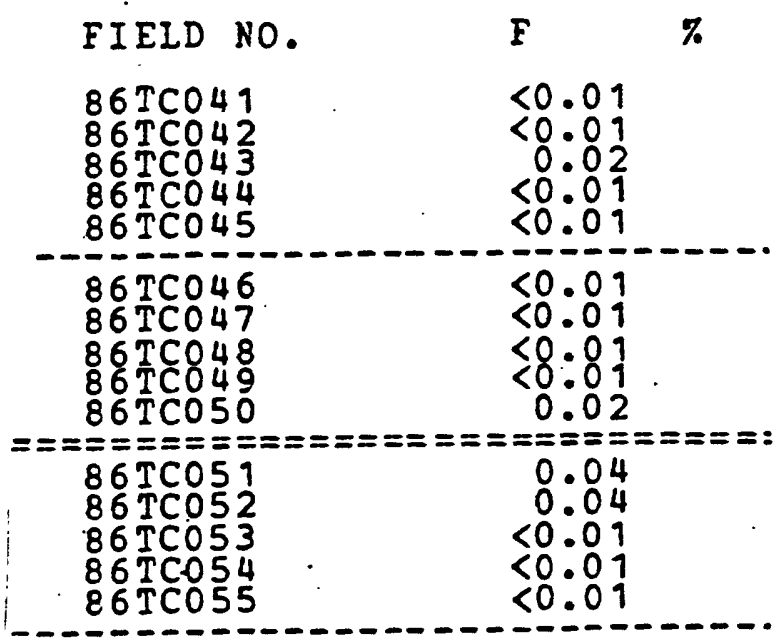

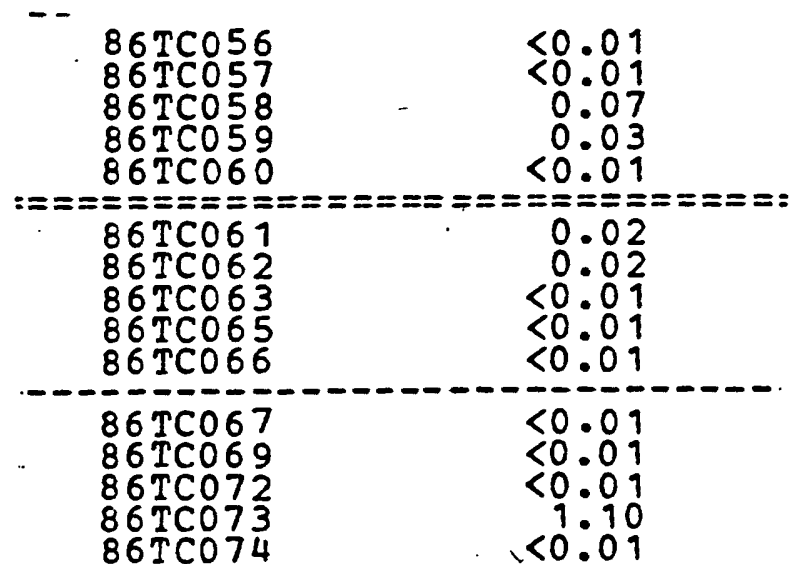

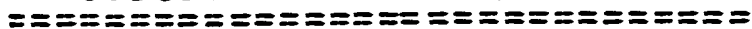
$86 \mathrm{TC} 077$

$86 \mathrm{TC} 078$

0.03

86 TC0 79

0.03

FIEID NO.

E

\%

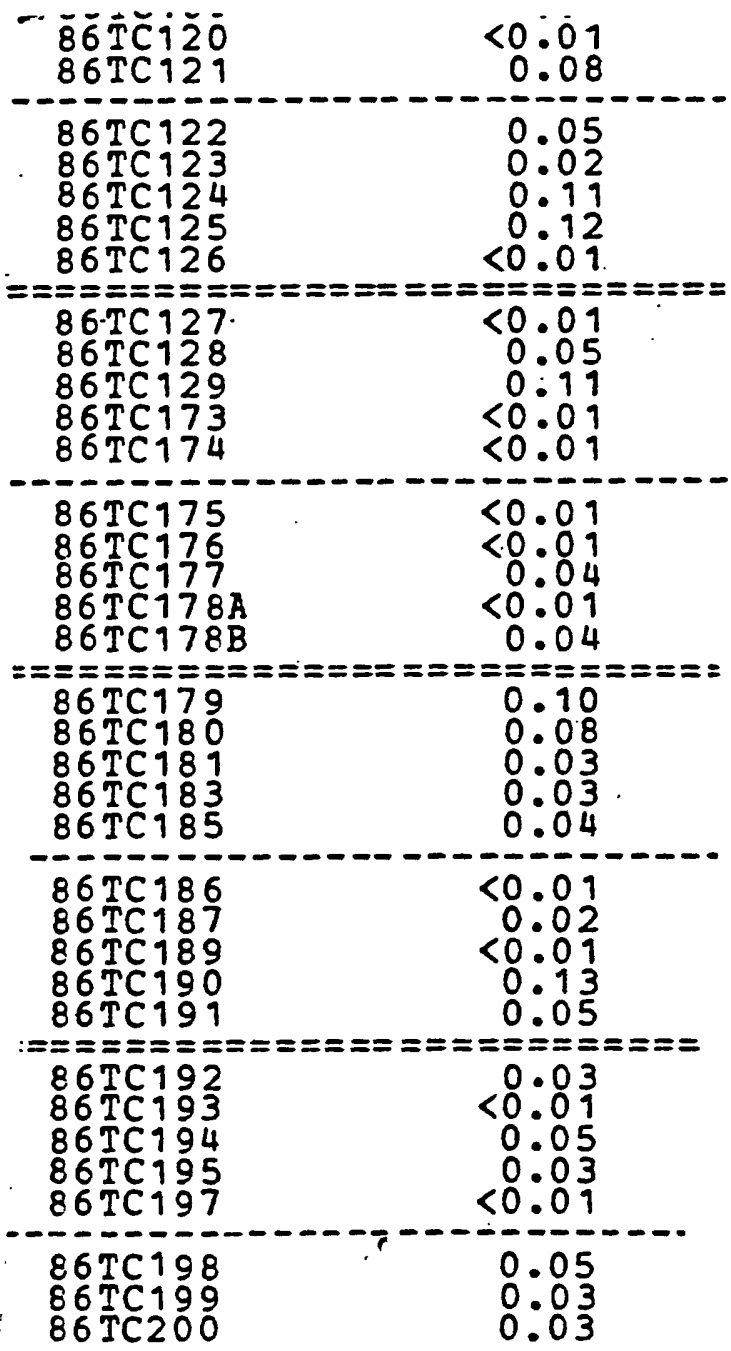




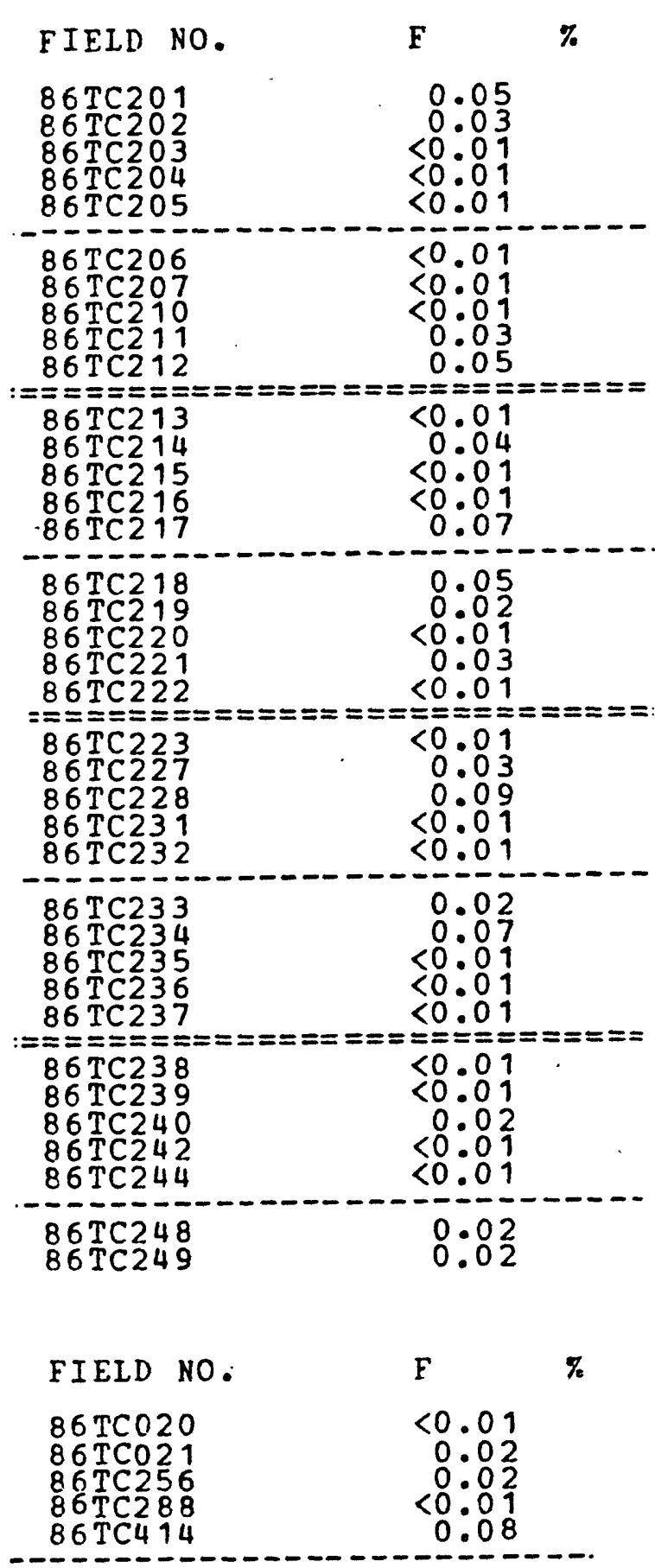

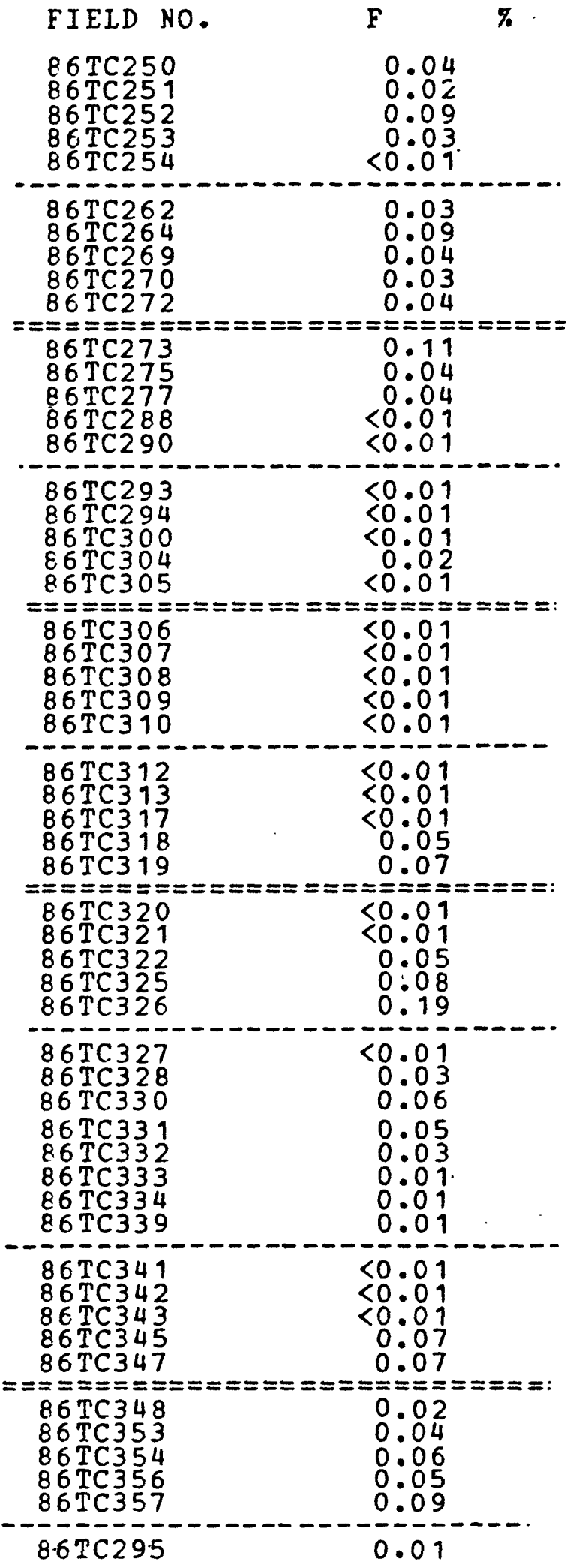




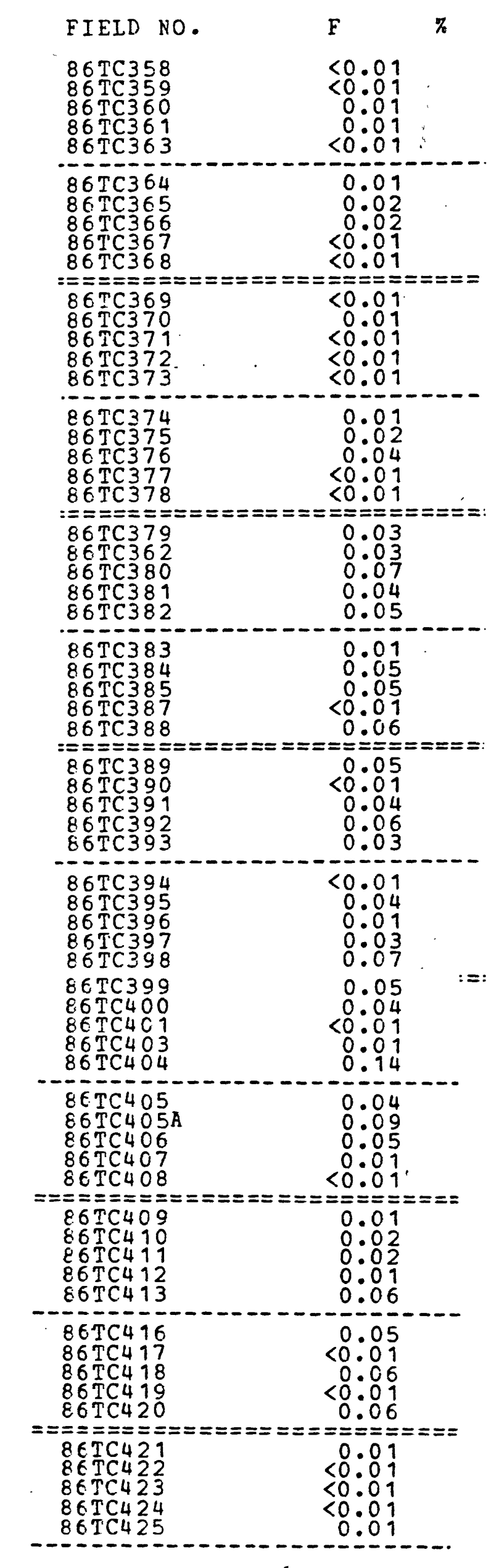

